Prepared in cooperation with the U.S. Army Corps of Engineers

\title{
Data Collected to Support Monitoring of Constructed Emergent Sandbar Habitat on the Missouri River Downstream from Gavins Point Dam, South Dakota and Nebraska, 2004-06
}

Open-File Report 2007-1056 
This page intentionally left blank. 


\section{Data Collected to Support Monitoring of Constructed Emergent Sandbar Habitat on the Missouri River Downstream from Gavins Point Dam, South Dakota and Nebraska, 2004-06}

By Ryan F. Thompson, Michaela R. Johnson, and Michael J. Andersen

Prepared in cooperation with the U.S. Army Corps of Engineers

Open-File Report 2007-1056 


\section{U.S. Department of the Interior DIRK KEMPTHORNE, Secretary}

\section{U.S. Geological Survey \\ Mark D. Myers, Director}

\section{U.S. Geological Survey, Reston, Virginia: 2007}

For product and ordering information:

World Wide Web: http://www.usgs.gov/pubprod

Telephone: 1-888-ASK-USGS

For more information on the USGS--the Federal source for science about the Earth, its natural and living resources, natural hazards, and the environment:

World Wide Web: http://www.usgs.gov

Telephone: 1-888-ASK-USGS

Any use of trade, product, or firm names is for descriptive purposes only and does not imply endorsement by the U.S. Government.

Although this report is in the public domain, permission must be secured from the individual copyright owners to reproduce any copyrighted materials contained within this report.

Suggested citation:

Thompson, R.F., Johnson, M.R., and Anderson, M.J., 2007, Data collected to support monitoring of constructed emergent sandbar habitat on the Missouri River downstream from Gavins Point Dam, South Dakota and Nebraska, 2004-06: U.S. Geological Survey Open-File Report 2007-1056, 178 p. 


\section{Contents}

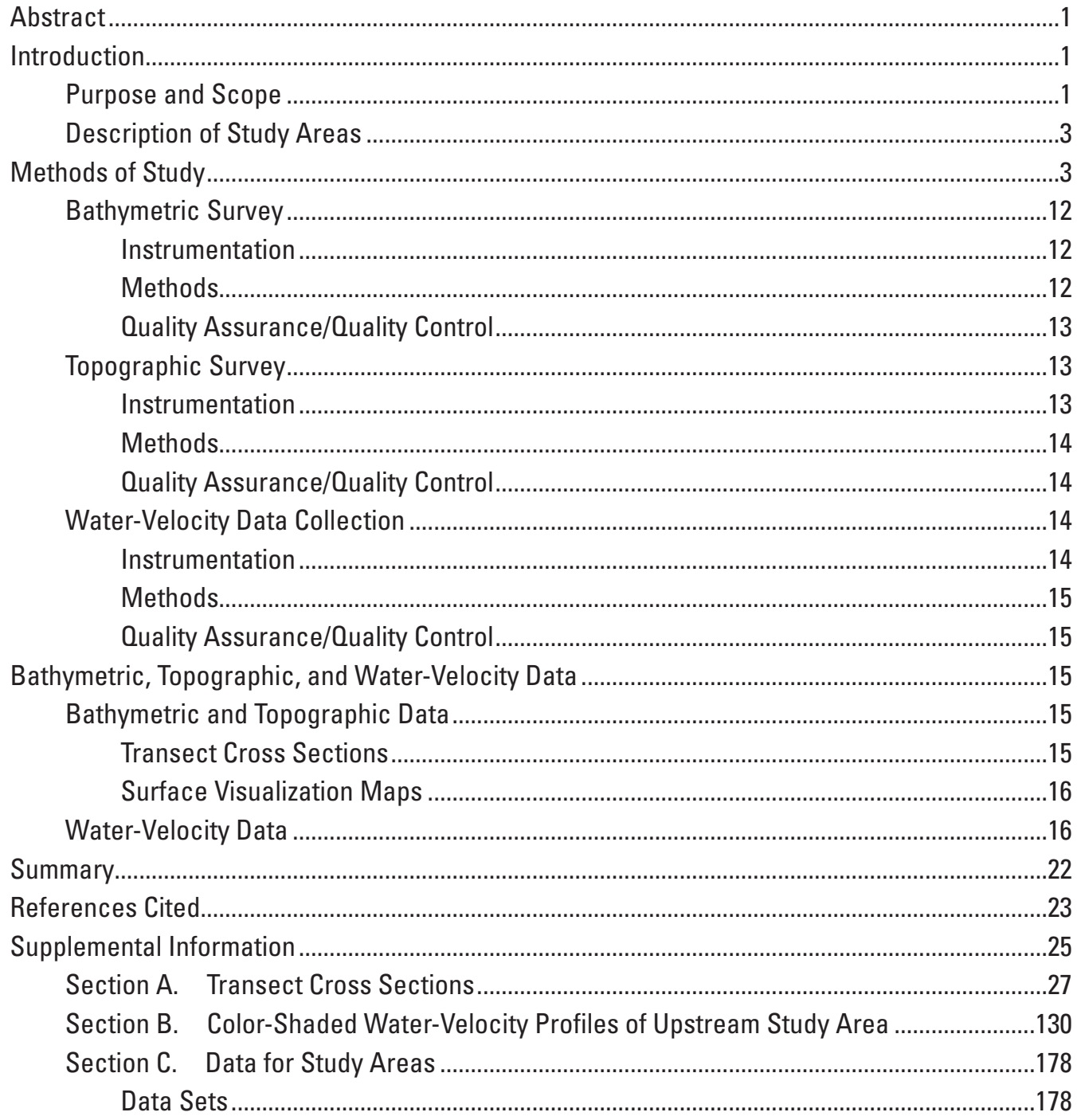

\section{Figures}

\section{1-3. Maps showing:}

1. Location of study areas

2. Location of data-collection transects in downstream study area (for the construction area at river mile 761.3)

3. Location of data-collection transects in upstream study area (for the construction area at river mile 769.8) 
4-6. Photographs showing:

4. Configuration of instruments used in bathymetric and water-velocity data collection.

5. Apparatus used to conduct bar check of echosounder transducer ......................13

6. Configuration of real-time kinematic global positioning system using backpack mount.

7-11. Maps showing:

7. Postconstruction land-surface elevation from transect 23 to 39 at the downstream study area near river mile 761.3.

8. Postconstruction land-surface elevation from transect 22 to 35 at the upstream study area near river mile 769.8

9. Difference between pre- and postconstruction land-surface elevation from transect 23 to 39 at the downstream study area near river mile 761.3.

10. Difference between pre- and postconstruction land-surface elevation from transect 22 to 35 at the upstream study area near river mile 769.8

11. Point water-velocity data in the upstream study area near river mile 769.8 ...........21

A1-A54. Location and cross section for downstream:
A1. Transect 7 .28
A2. Transect 10. .29
A3. Transect 12 . 30
A4. Transect 13. 31
A5. Transect 14. ...32
A6. Transect 15. .33
A7. Transect 18. 34
A8. Transect 20. .35
A9. Transect 21 ...36
A10. Transect 22

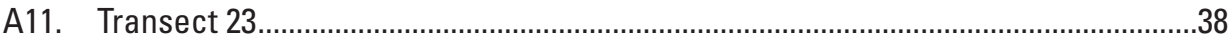

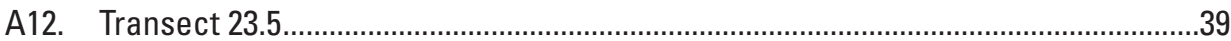

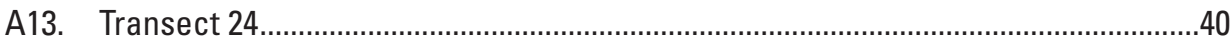

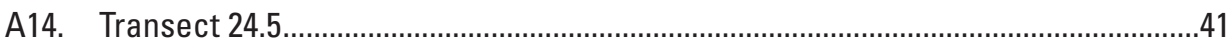

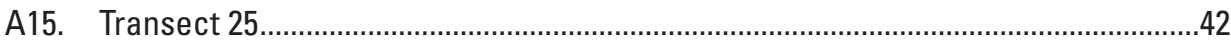
A16. Transect 25.5
A17. Transect 26
A18. Transect 26.5

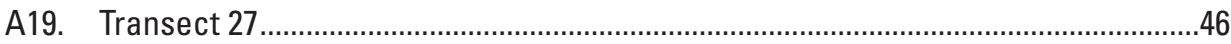

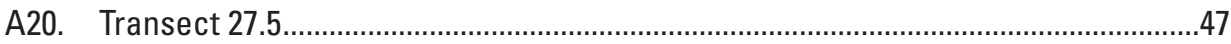
A21. Transect 28
A22. Transect 28.5
A23. Transect 29
A24. Transect 29.5

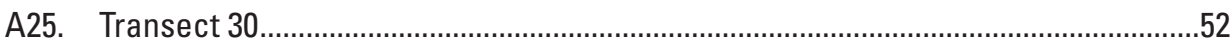
A26. Transect 30.5

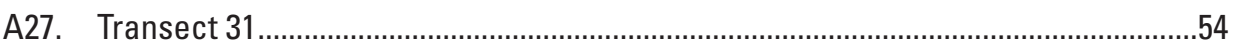

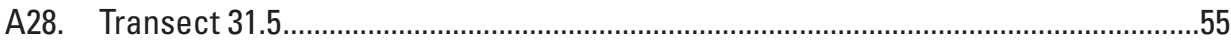


A29. Transect 32

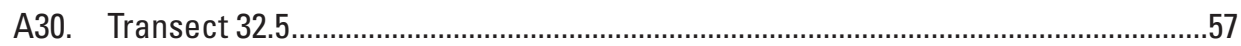

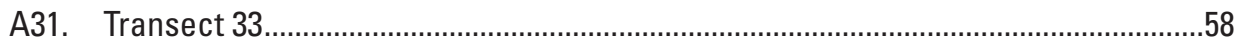

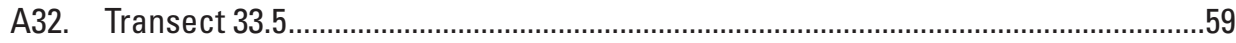

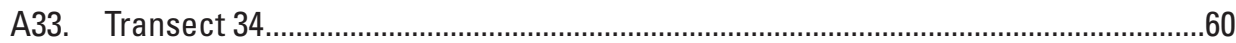

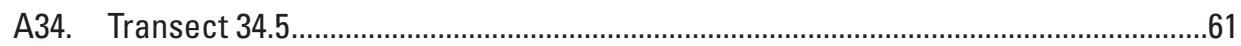

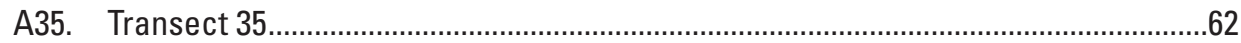

A36. Transect 35.5

A37. Transect 36

A38. Transect 36.5

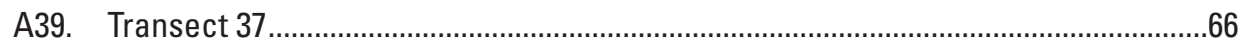

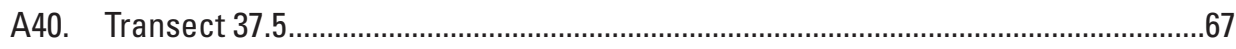

A41. Transect 38

A42. Transect 38.5

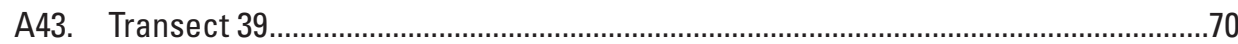

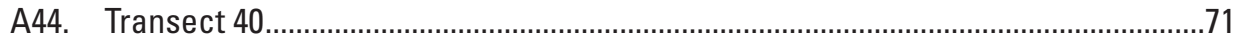

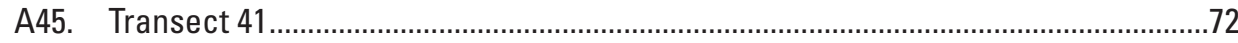

A46. Transect 42

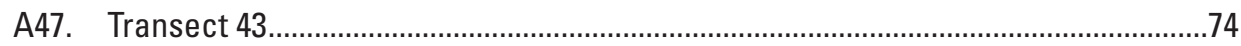

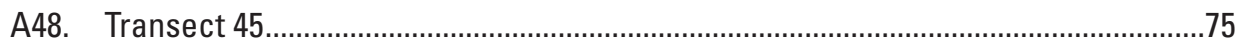

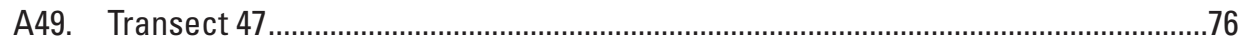

A50. Transect 50

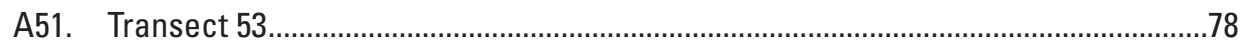

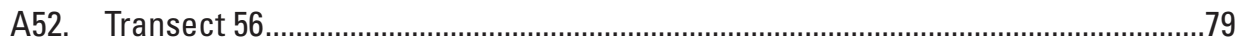

A53. Transect 58

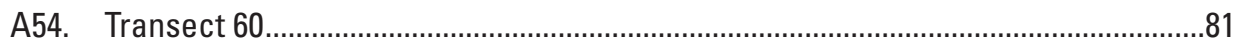

A55-A102. Location and cross section for upstream:

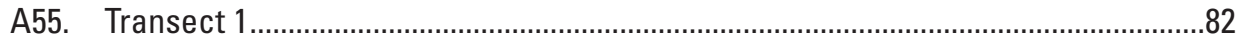

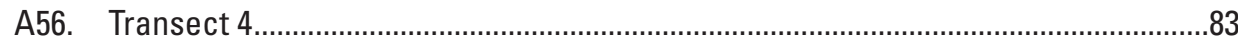

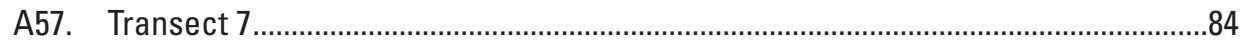

A58. Transect 10

A59. Transect 13

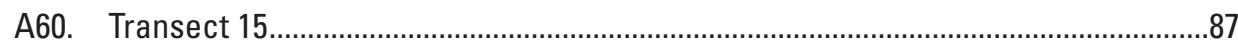

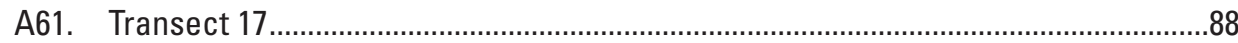

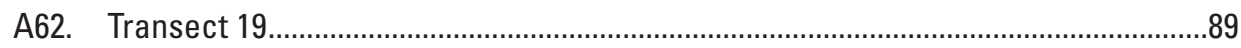

A63. Transect 20

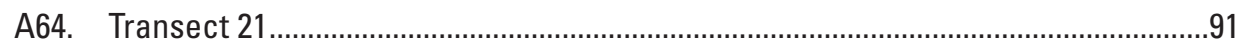

A65. Transect 22

A66. Transect 22.5

A67. Transect 23

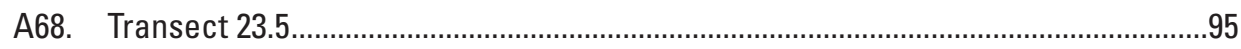

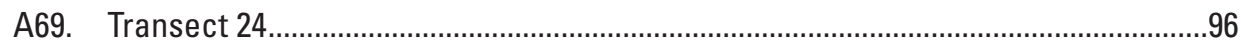

A70. Transect 24.5

A71. Transect 25 
A72. Transect 25.5

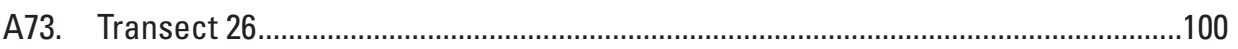

A74. Transect 26.5

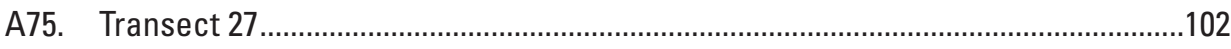

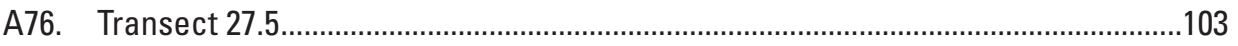

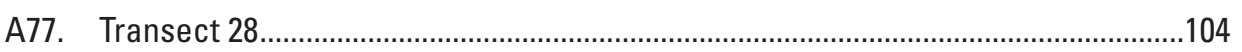

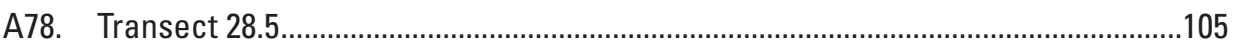

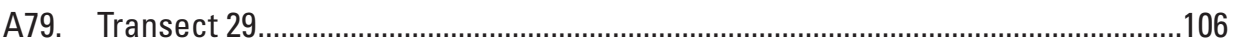

A80. Transect 29.5

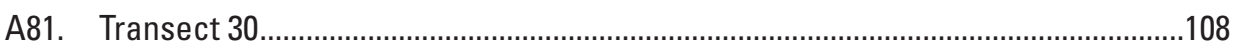

A82. Transect 30.5

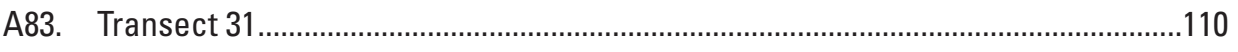

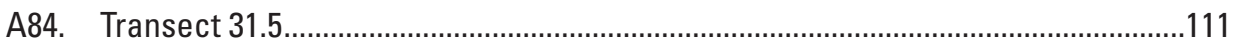

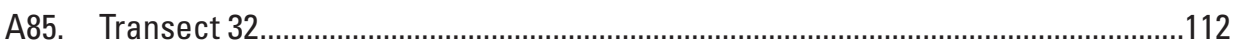

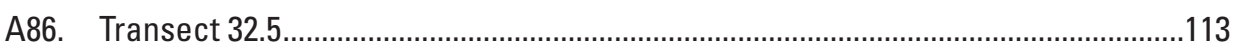

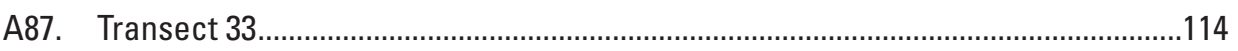

A88. Transect 33.5

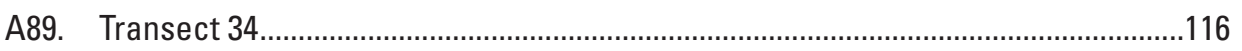

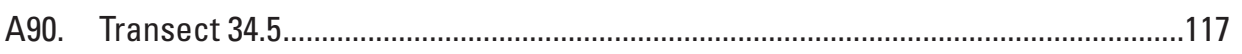

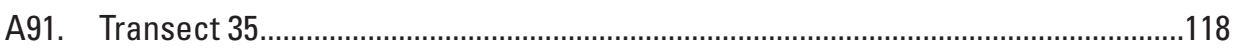

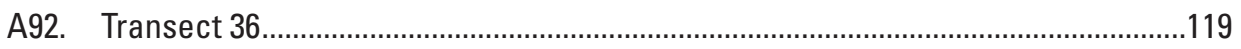

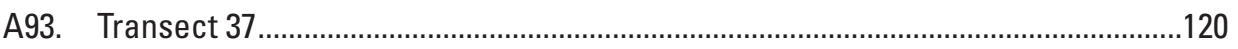

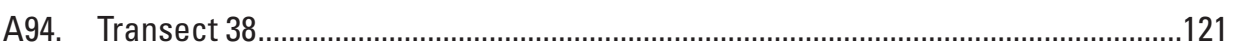

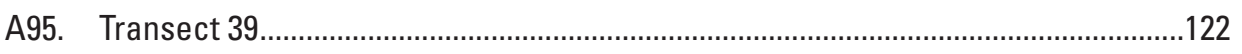

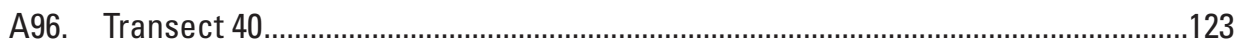

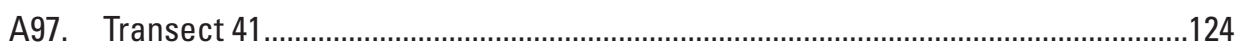

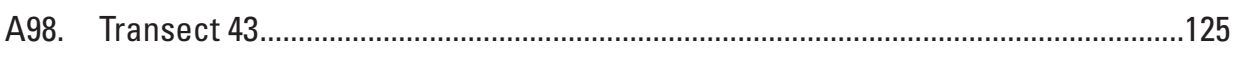

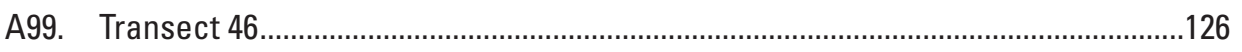

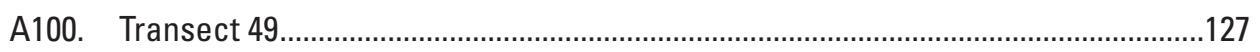

A101. Transect 52

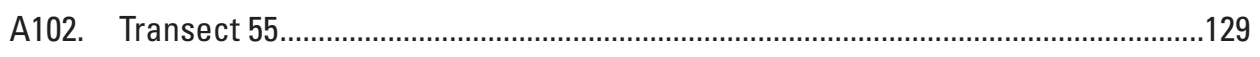

B1-B47. Location and velocity profile for upstream:

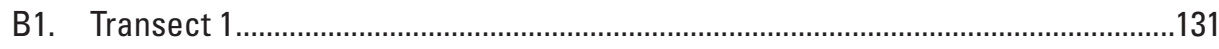

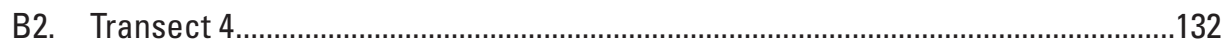

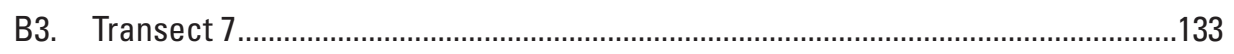

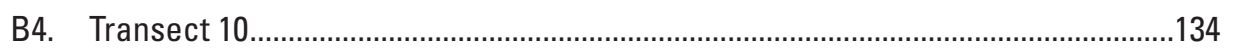

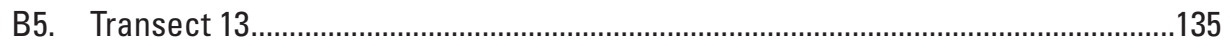

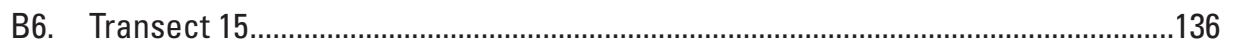

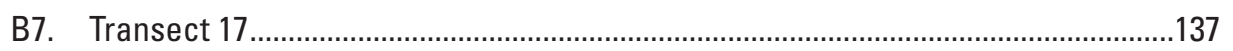

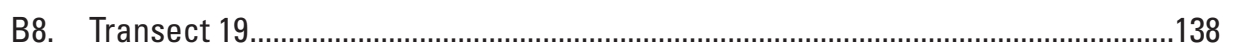

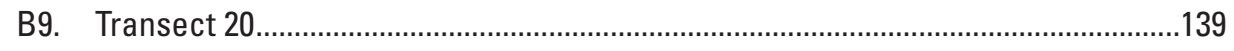

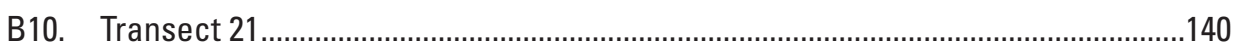

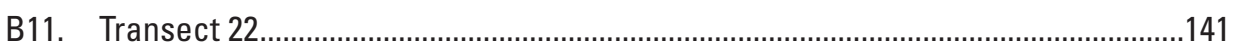

B12. Transect 22.5 


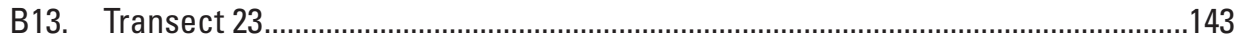

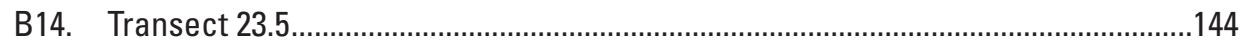

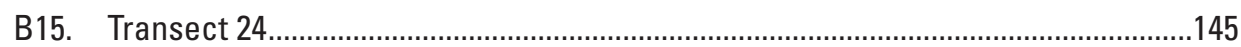

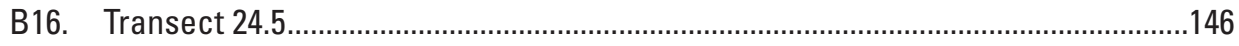

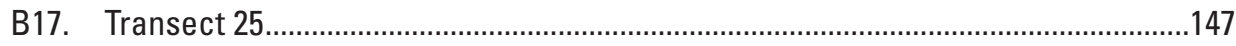

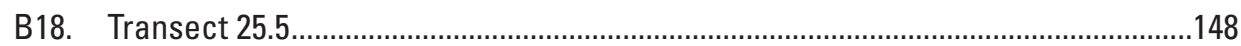

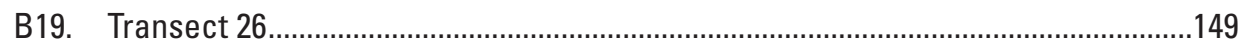

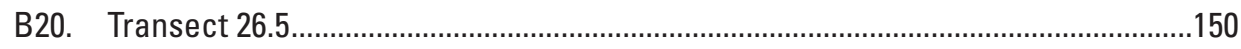

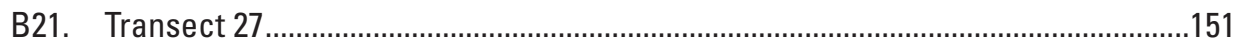

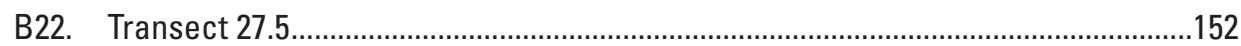

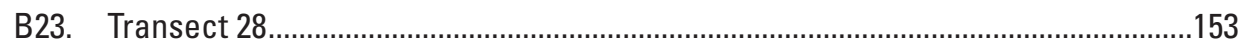

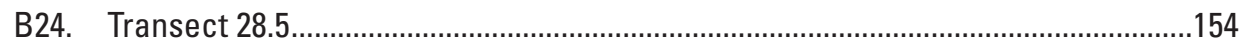

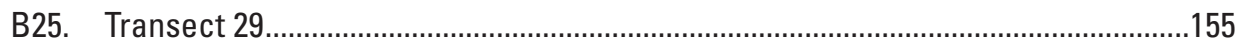

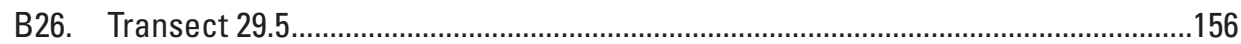

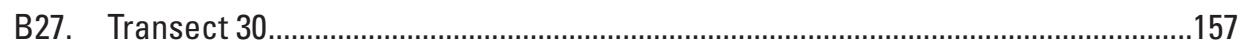

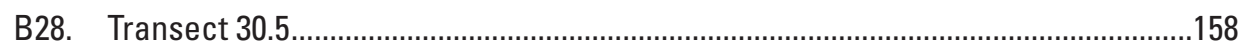

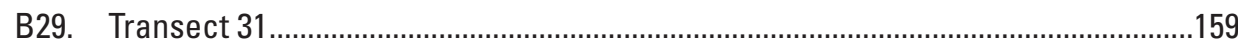

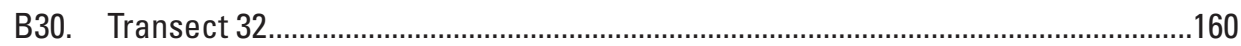

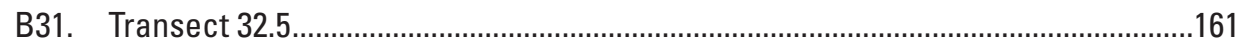

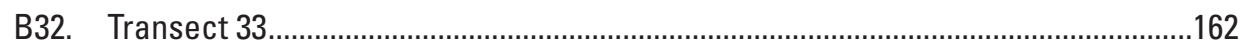

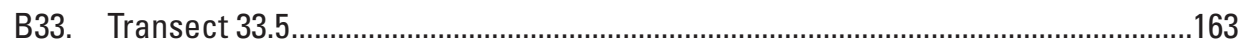

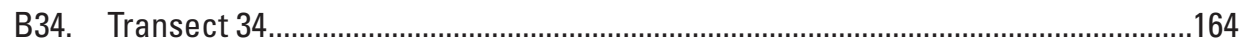

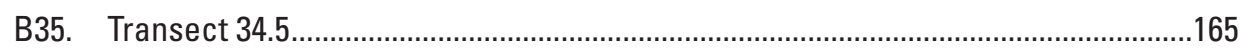

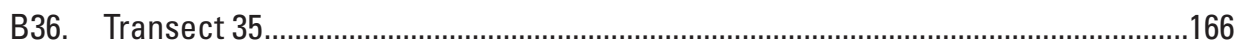

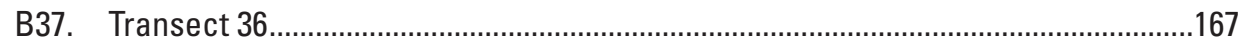

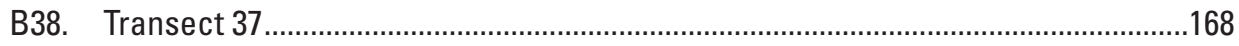

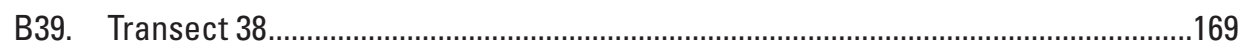

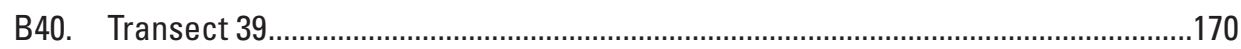

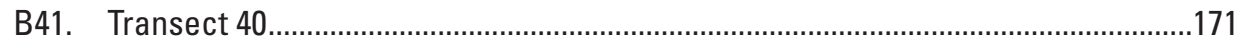

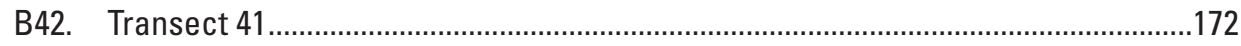

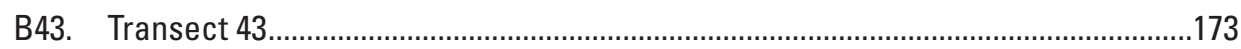

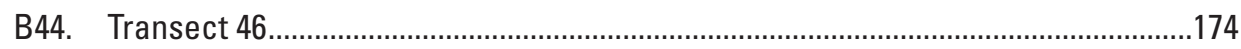

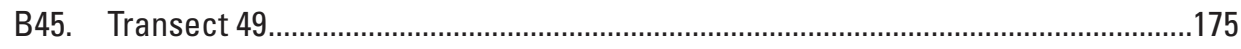

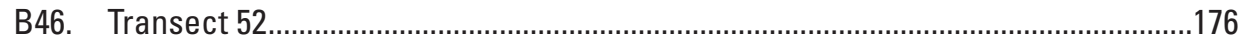

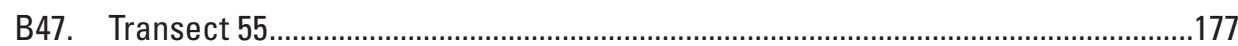

\section{Tables}

1. Transects specified for pre- and postconstruction data collection....................................6

2. Coordinates of transect end points, in Universal Transverse Mercator feet, Zone 14, and North American Datum of 1983....................................................................8

3. National Geodetic Survey benchmark relative accuracy and estimated spatial accuracy 


\section{Conversion Factors}

\begin{tabular}{lcl} 
Multiply & By & To obtain \\
\hline & Length & \\
\hline inch (in.) & 2.54 & centimeter $(\mathrm{cm})$ \\
inch (in.) & 25.4 & millimeter $(\mathrm{mm})$ \\
foot (ft) & 0.3048 & meter $(\mathrm{m})$ \\
mile (mi) & 1.609 & kilometer $(\mathrm{km})$ \\
\hline & Flow rate & \\
\hline foot per second $(\mathrm{ft} / \mathrm{s})$ & 0.3048 & meter per second $(\mathrm{m} / \mathrm{s})$ \\
cubic foot per second $\left(\mathrm{ft}^{3} / \mathrm{s}\right)$ & 0.02832 & cubic meter per second $\left(\mathrm{m}^{3} / \mathrm{s}\right)$ \\
\hline
\end{tabular}

Vertical coordinate information is referenced to the National Geodetic Vertical Datum of 1929 (NGVD 29).

Horizontal coordinate information is referenced to either the North American Datum of 1983 (NAD 83) or the North American Datum of 1927 (NAD 27). See metadata of the individual data sets for specific horizontal datum.

\section{Acronyms}

$\begin{array}{ll}\text { ADCP } & \text { Acoustic Doppler Current Profiler } \\ \text { ADV } & \text { Acoustic Doppler Velocimeter } \\ \text { DGPS } & \text { differential global positioning system } \\ \text { DOO } & \text { digital orthophoto quadrangle } \\ \text { ESH } & \text { emergent sandbar habitat } \\ \text { GIS } & \text { geographic information system } \\ \text { GPS } & \text { global positioning system } \\ \text { NGS } & \text { National Geodetic Survey } \\ \text { OA/OC } & \text { quality-assurance/quality-control } \\ \text { RM } & \text { river mile } \\ \text { RTK } & \text { real-time kinematic } \\ \text { TIN } & \text { triangular irregular network } \\ \text { USACE } & \text { U.S. Army Corps of Engineers } \\ \text { USGS } & \text { U.S. Geological Survey } \\ \text { UTM } & \text { Universal Transverse Mercator }\end{array}$




\title{
Data Collected to Support Monitoring of Constructed Emergent Sandbar Habitat on the Missouri River Downstream from Gavins Point Dam, South Dakota and Nebraska, 2004-06
}

\author{
By Ryan F. Thompson, Michaela R. Johnson, and Michael J. Andersen
}

\section{Abstract}

The U.S. Army Corps of Engineers has constructed emergent sandbar habitat on sections of the Missouri River bordering South Dakota and Nebraska downstream from Gavins Point Dam to create and enhance habitat for threatened and endangered bird species. Two areas near river miles 761.3 and 769.8 were selected for construction of emergent sandbar habitat. Pre- and postconstruction data were collected by the U.S. Geological Survey, in cooperation with the U.S. Army Corps of Engineers, to evaluate the success of the habitat management techniques. Data collected include pre- and postconstruction channel-geometry data (bathymetric and topographic) for areas upstream from, downstream from, and within each construction site. Water-velocity data were collected for selected parts of the site near river mile 769.8. Instruments and methods used in data collection, as well as quality-assurance and quality-control measures, are described. Geospatial channel-geometry data are presented for transects of the river channel as cross sections and as geographical information system shapefiles. Geospatial land-surface elevation data are provided for part of each site in the form of a color-shaded relief map. Geospatial water-velocity data also are provided as color-shaded maps and geographical information system shapefiles.

\section{Introduction}

The U.S. Army Corps of Engineers (USACE) authorized a project to alter part of the Missouri River channel downstream from Gavins Point Dam in South Dakota and Nebraska. Channel alterations involved construction of emergent sandbar habitat $(\mathrm{ESH})$ for endangered least terns and threatened piping plovers as outlined in the 2000 Biological Opinion (U.S. Fish and Wildlife Service, 2000) and the amended 2003
Biological Opinion (2003 BiOp) (U.S. Fish and Wildlife Service, 2003). The $2003 \mathrm{BiOp}$ also mandates an intensive program to monitor and evaluate the success of habitat management techniques used by the USACE to meet specific habitat goals. Therefore, to assess the physical and ecological effects of the channel alterations, accurate information was required to define the Missouri River channel geometry prior to and after construction of the project. Two areas were selected by the USACE for creation and enhancement of ESH (fig. 1). The U.S. Geological Survey (USGS), in cooperation with the USACE, collected data to document the pre- and postconstruction channel geometry within the two areas. The channel alterations were completed in the fall of 2004 and spring of 2005 for the downstream and upstream ESH sites, respectively. Preconstruction data were collected during the fall of 2004 and postconstruction data were collected during November 2005 and February 2006.

\section{Purpose and Scope}

The purpose of this report is to describe the methods used in data collection and to present pre- and postconstruction channel-geometry data collected from hydrographic (bathymetric and topographic) surveys and postconstruction water-velocity data for ESH on the Missouri River downstream from Gavins Point Dam, located on the South Dakota and Nebraska State line, for 2004-06. Preand postconstruction channel-geometry data are provided in both raw and processed forms for each of the two ESH sites. Geospatial channel-geometry data are presented for transects of the river channel as cross sections and as geographical information system (GIS) shapefiles. Geospatial land-surface data are provided for part of each site in the form of a colorshaded relief map. Postconstruction geospatial water-velocity data for selected transects also are provided as color-shaded maps and GIS shapefiles. 


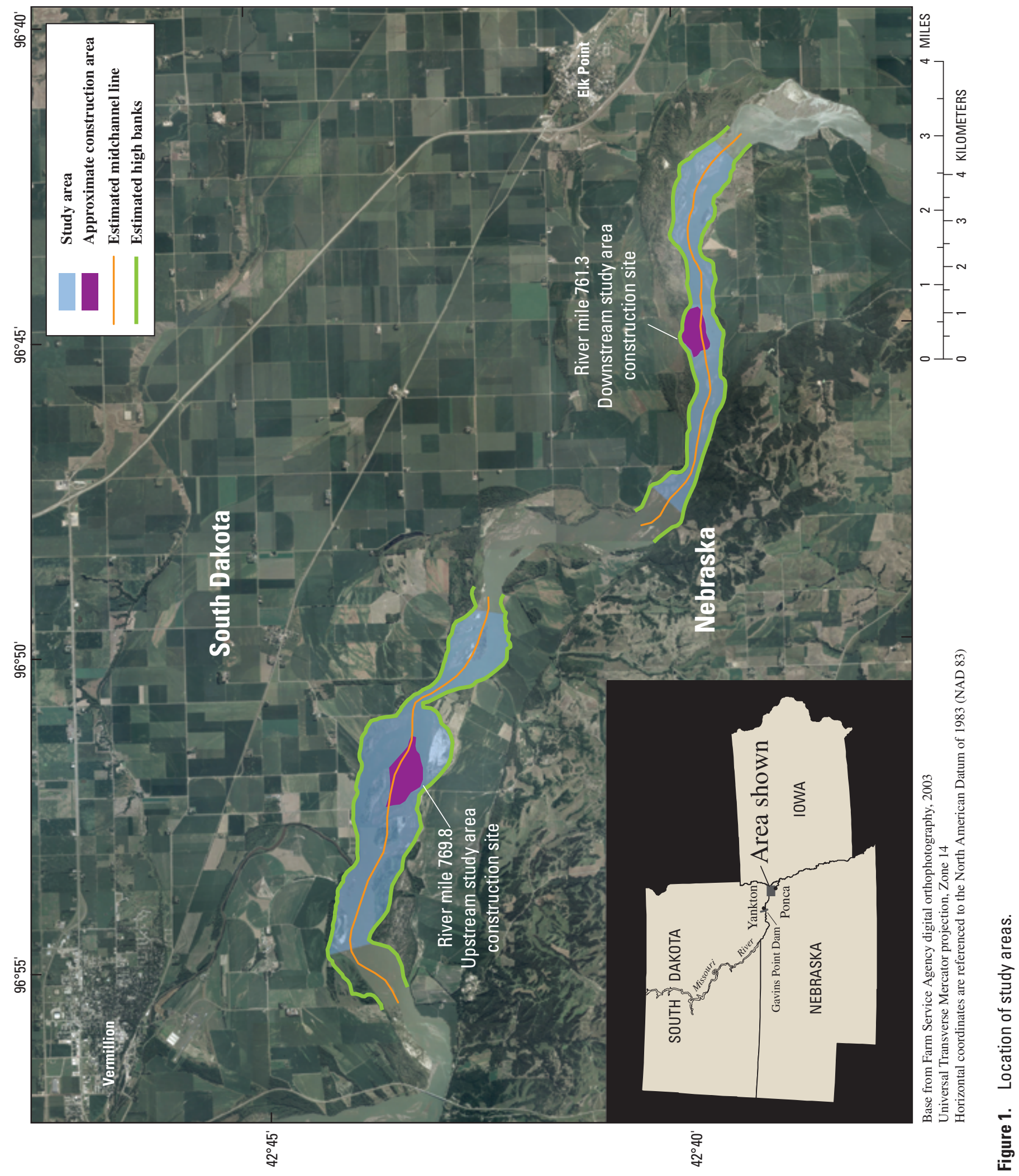




\section{Description of Study Areas}

Gavins Point Dam is located approximately 3 miles (mi) west of Yankton, South Dakota, on a part of the Missouri River that forms the South Dakota-Nebraska State line (fig. 1). The part between Gavins Point Dam and Ponca, Nebraska, is known as the lower district of the Missouri National Recreation River, where river conditions remain in a relatively natural state. The areas selected for creation and enhancement of ESH are located near river mile (RM) 761.3 (fig. 2) and RM 769.8 (fig. 3) near the South Dakota towns of Elk Point and Vermillion, respectively (fig. 1).

The USACE specified that for each ESH site, preconstruction channel-geometry information was required for a reach extending from $2 \mathrm{mi}$ upstream to $2 \mathrm{mi}$ downstream from the site boundaries. Thus, each of the study areas is composed of a construction area and the 2-mi upstream and downstream buffers adjacent to the construction area.

Despite the impoundments located upstream from Gavins Point Dam, the Missouri River between Gavins Point Dam and Ponca, Nebraska, still possesses several of the characteristics of a "wild" river. As such, certain natural phenomena can be assumed, including shifting sandbars, a primary channel with swift currents, and islands that erode and re-deposit downstream. A major tributary, the Vermillion River, enters the Missouri River in the upstream study area (fig. 3), and several other draws and sloughs contribute flow on a seasonal basis. The width of the channel varies from approximately 750 feet (ft) to more than $6,000 \mathrm{ft}$, and water depths during data collection occasionally reached $30 \mathrm{ft}$. Although flows were less than 12,000 cubic feet per second $\left(\mathrm{ft}^{3} / \mathrm{s}\right)$ during data collection, considerable shifting of sandbars was observed in this dynamic system.

\section{Methods of Study}

For preconstruction data collection, a series of transects were laid out every $500 \mathrm{ft}$ approximately perpendicular to the general flow direction of the river. A slightly revised set of transects was used for postconstruction channel-geometry data collection. To better define channel geometry near the ESH sites, the postconstruction transects were spaced more widely at the upstream and downstream ends of the study areas, and additional transects were added between the original transects throughout each construction area. All transects for which data are presented in this report are shown in figures 2 and 3 and listed in table 1 . Channel-geometry data were collected approximately every $2 \mathrm{ft}$ along the length of each transect from high bank to high bank and deviated no more than 10 $\mathrm{ft}$ on either side of the transect. The coordinates of transect end points, in Universal Transverse Mercator (UTM) feet, Zone 14, and the North American Datum of 1983 (NAD 83) are listed in table 2. 


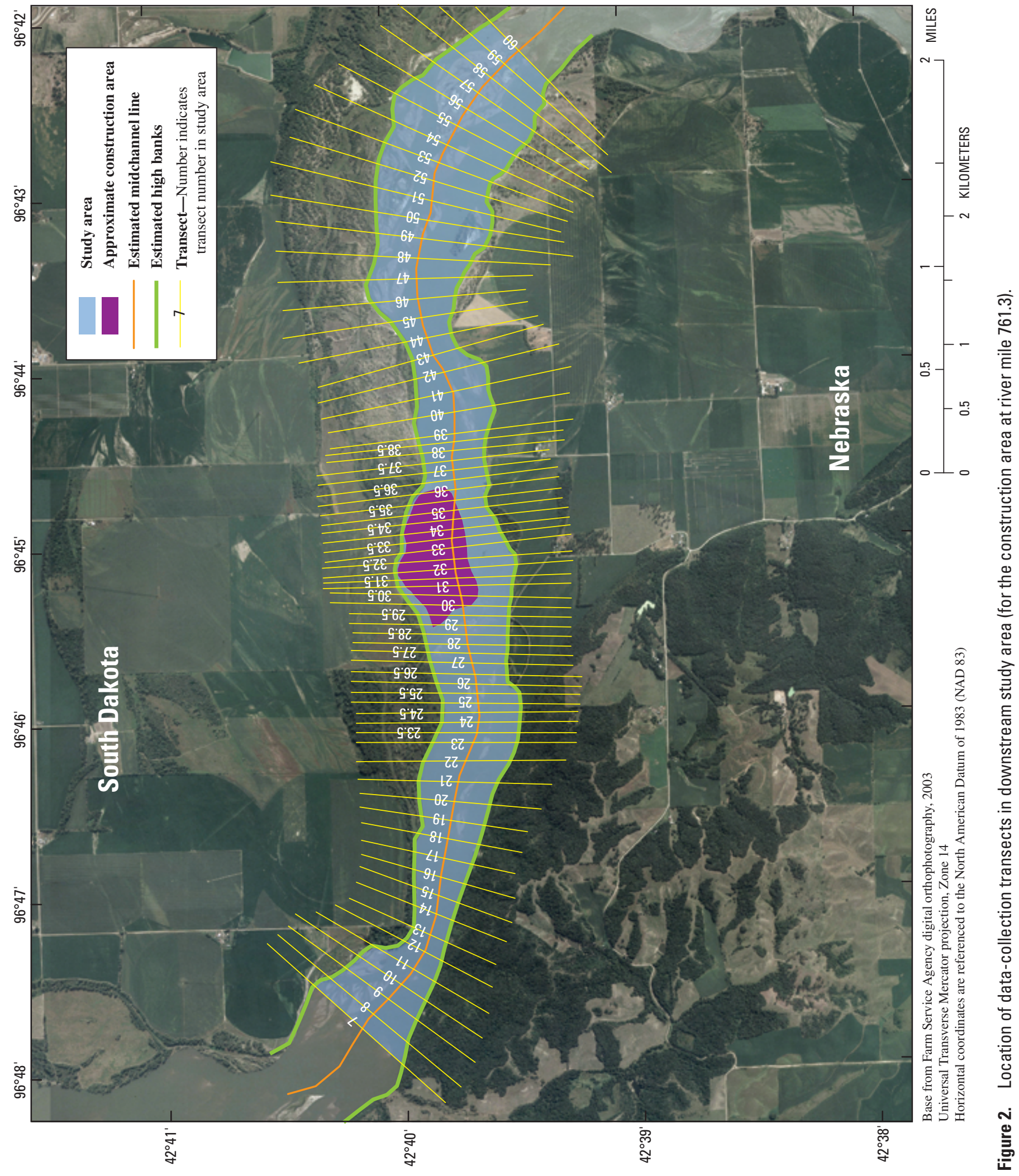




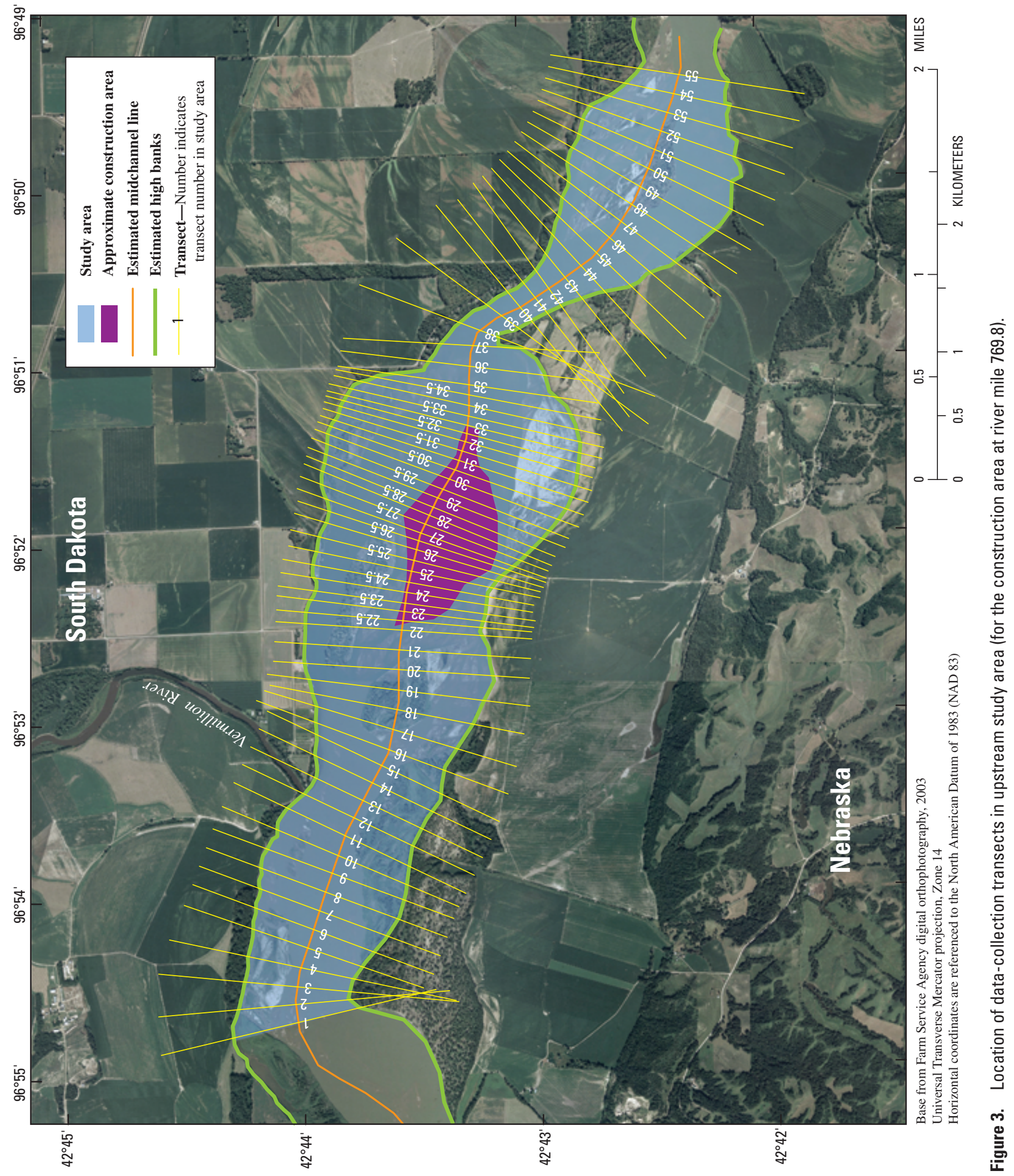


Table 1. Transects specified for pre- and postconstruction data collection.

[RM, river mile]

\begin{tabular}{|c|c|}
\hline Preconstruction & Postconstruction \\
\hline \multicolumn{2}{|c|}{ Downstream study area (encompassing RM 761.3) } \\
\hline 7 & 7 \\
\hline 8 & 10 \\
\hline 9 & 12 \\
\hline 10 & 13 \\
\hline 11 & 14 \\
\hline 12 & 15 \\
\hline 13 & 18 \\
\hline 14 & 20 \\
\hline 15 & 21 \\
\hline 16 & 22 \\
\hline 17 & 23 \\
\hline 18 & 23.5 \\
\hline 19 & 24 \\
\hline 20 & 24.5 \\
\hline 21 & 25 \\
\hline 22 & 25.5 \\
\hline 23 & 26 \\
\hline 24 & 26.5 \\
\hline 25 & 27 \\
\hline 26 & 27.5 \\
\hline 27 & 28 \\
\hline 28 & 28.5 \\
\hline 29 & 29 \\
\hline 30 & 29.5 \\
\hline 31 & 30 \\
\hline 32 & 30.5 \\
\hline 33 & 31 \\
\hline
\end{tabular}

\begin{tabular}{|c|c|}
\hline Preconstruction & Postconstruction \\
\hline 34 & 31.5 \\
\hline 35 & 32 \\
\hline 36 & 32.5 \\
\hline 37 & 33 \\
\hline 38 & 33.5 \\
\hline 39 & 34 \\
\hline 40 & 34.5 \\
\hline 41 & 35 \\
\hline 42 & 35.5 \\
\hline 43 & 36 \\
\hline 44 & 36.5 \\
\hline 45 & 37 \\
\hline 46 & 37.5 \\
\hline 47 & 38 \\
\hline 48 & 38.5 \\
\hline 49 & 39 \\
\hline 50 & 40 \\
\hline 51 & 41 \\
\hline 52 & 42 \\
\hline 53 & 43 \\
\hline 54 & 45 \\
\hline 55 & 47 \\
\hline 56 & 50 \\
\hline 57 & 53 \\
\hline 58 & 56 \\
\hline 59 & 58 \\
\hline 60 & 60 \\
\hline Total: 54 & 54 \\
\hline
\end{tabular}


Table 1. Transects specified for pre- and postconstruction data collection.-Continued [RM, river mile]

\begin{tabular}{|c|c|}
\hline Preconstruction & Postconstruction \\
\hline \multicolumn{2}{|c|}{ Upstream study area (encompassing RM 769.8) } \\
\hline 1 & 1 \\
\hline 2 & 4 \\
\hline 3 & 7 \\
\hline 4 & 10 \\
\hline 5 & 13 \\
\hline 6 & 15 \\
\hline 7 & 17 \\
\hline 8 & 19 \\
\hline 9 & 20 \\
\hline 10 & 21 \\
\hline 11 & 22 \\
\hline 12 & 22.5 \\
\hline 13 & 23 \\
\hline 14 & 23.5 \\
\hline 15 & 24 \\
\hline 16 & 24.5 \\
\hline 17 & 25 \\
\hline 18 & 25.5 \\
\hline 19 & 26 \\
\hline 20 & 26.5 \\
\hline 21 & 27 \\
\hline 22 & 27.5 \\
\hline 23 & 28 \\
\hline 24 & 28.5 \\
\hline 25 & 29 \\
\hline 26 & 29.5 \\
\hline 27 & 30 \\
\hline
\end{tabular}

\begin{tabular}{|c|c|}
\hline Preconstruction & Postconstruction \\
\hline 28 & 30.5 \\
\hline 29 & 31 \\
\hline 30 & 31.5 \\
\hline 31 & 32 \\
\hline 32 & 32.5 \\
\hline 33 & 33 \\
\hline 34 & 33.5 \\
\hline 35 & 34 \\
\hline 36 & 34.5 \\
\hline 37 & 35 \\
\hline 38 & 36 \\
\hline 39 & 37 \\
\hline 40 & 38 \\
\hline 41 & 39 \\
\hline 42 & 40 \\
\hline 43 & 41 \\
\hline 44 & 43 \\
\hline 45 & 46 \\
\hline 46 & 49 \\
\hline 47 & 52 \\
\hline 48 & 55 \\
\hline 49 & \\
\hline 50 & \\
\hline 51 & \\
\hline 52 & \\
\hline 53 & \\
\hline 54 & \\
\hline 55 & \\
\hline Total: 55 & 48 \\
\hline
\end{tabular}


Table 2. Coordinates of transect end points, in Universal Transverse Mercator feet, Zone 14, and North American Datum of 1983.

[ft, feet; RM, river mile]

\begin{tabular}{|c|c|c|c|c|}
\hline \multirow[b]{2}{*}{ Transect number } & \multicolumn{2}{|c|}{ End point in South Dakota } & \multicolumn{2}{|c|}{ End point in Nebraska } \\
\hline & $\begin{array}{c}\text { Easting } \\
(\mathrm{ft})\end{array}$ & $\begin{array}{c}\text { Northing } \\
\text { (ft) }\end{array}$ & $\begin{array}{c}\text { Easting } \\
\text { (ft) }\end{array}$ & $\begin{array}{c}\text { Northing } \\
\text { (ft) }\end{array}$ \\
\hline \multicolumn{5}{|c|}{ Downstream study area (encompassing RM 761.3) } \\
\hline 7 & $2,235,057.57$ & $15,509,263.54$ & $2,230,986.84$ & $15,504,700.72$ \\
\hline 8 & $2,235,281.23$ & $15,508,995.13$ & $2,231,389.43$ & $15,504,298.13$ \\
\hline 9 & $2,235,504.91$ & $15,508,726.73$ & $2,232,015.71$ & $15,503,940.25$ \\
\hline 10 & $2,235,818.04$ & $15,508,547.79$ & $2,232,641.96$ & $15,503,761.35$ \\
\hline 11 & $2,235,862.75$ & $15,508,010.98$ & $2,233,268.24$ & $15,503,582.41$ \\
\hline 12 & $2,236,041.69$ & $15,507,518.92$ & $2,233,939.23$ & $15,503,492.94$ \\
\hline 13 & $2,236,220.63$ & $15,507,161.05$ & $2,234,520.76$ & $15,503,403.47$ \\
\hline 14 & $2,236,623.22$ & $15,506,982.14$ & $2,235,102.29$ & $15,503,135.07$ \\
\hline 15 & $2,237,025.84$ & $15,506,892.67$ & $2,235,594.38$ & $15,503,000.85$ \\
\hline 16 & $2,237,338.96$ & $15,506,847.92$ & $2,236,220.63$ & $15,502,911.42$ \\
\hline 17 & $2,237,696.84$ & $15,506,847.92$ & $2,236,846.90$ & $15,502,911.42$ \\
\hline 18 & $2,238,144.15$ & $15,506,847.92$ & $2,237,383.68$ & $15,502,732.48$ \\
\hline 19 & $2,238,546.77$ & $15,506,892.67$ & $2,237,920.49$ & $15,502,598.26$ \\
\hline 20 & $2,238,904.64$ & $15,506,892.67$ & $2,238,502.02$ & $15,502,240.39$ \\
\hline 21 & $2,239,262.48$ & $15,506,937.39$ & $2,239,128.30$ & $15,501,972.01$ \\
\hline 22 & $2,239,709.83$ & $15,506,982.14$ & $2,239,754.58$ & $15,501,614.14$ \\
\hline 23 & $2,240,201.88$ & $15,506,982.14$ & $2,240,201.88$ & $15,501,345.74$ \\
\hline 23.5 & $2,240,451.88$ & $15,506,982.14$ & $2,240,451.88$ & $15,501,345.74$ \\
\hline 24 & $2,240,693.94$ & $15,506,982.14$ & $2,240,738.69$ & $15,501,301.02$ \\
\hline 24.5 & $2,240,943.94$ & $15,506,984.11$ & $2,240,988.69$ & $15,501,302.99$ \\
\hline 25 & $2,241,230.75$ & $15,507,116.33$ & $2,241,186.04$ & $15,501,256.27$ \\
\hline 25.5 & $2,241,480.75$ & $15,507,114.42$ & $2,241,436.03$ & $15,501,254.36$ \\
\hline 26 & $2,241,767.56$ & $15,507,116.33$ & $2,241,633.34$ & $15,501,211.55$ \\
\hline 26.5 & $2,242,017.50$ & $15,507,110.65$ & $2,241,883.28$ & $15,501,205.87$ \\
\hline 27 & $2,242,304.34$ & $15,507,116.33$ & $2,242,170.15$ & $15,501,435.20$ \\
\hline 27.5 & $2,242,554.27$ & $15,507,110.43$ & $2,242,420.08$ & $15,501,429.30$ \\
\hline 28 & $2,242,751.68$ & $15,507,071.61$ & $2,242,662.21$ & $15,501,479.92$ \\
\hline 28.5 & $2,243,001.65$ & $15,507,067.61$ & $2,242,912.18$ & $15,501,475.92$ \\
\hline 29 & $2,243,243.72$ & $15,507,161.05$ & $2,243,154.27$ & $15,501,479.92$ \\
\hline 29.5 & $2,243,493.71$ & $15,507,157.11$ & $2,243,404.24$ & $15,501,475.99$ \\
\hline 30 & $2,243,780.55$ & $15,507,653.14$ & $2,243,646.37$ & $15,501,479.92$ \\
\hline 30.5 & $2,244,030.49$ & $15,507,647.71$ & $2,243,896.31$ & $15,501,474.49$ \\
\hline 31 & $2,244,138.43$ & $15,507,832.07$ & $2,244,272.61$ & $15,501,479.92$ \\
\hline 31.5 & $2,244,276.34$ & $15,507,855.50$ & $2,244,522.56$ & $15,501,485.20$ \\
\hline 32 & $2,244,406.83$ & $15,507,832.07$ & $2,244,854.14$ & $15,501,435.20$ \\
\hline
\end{tabular}


Table 2. Coordinates of transect end points, in Universal Transverse Mercator feet, Zone 14, and North American Datum of 1983.-Continued

[ft, feet; RM, river mile]

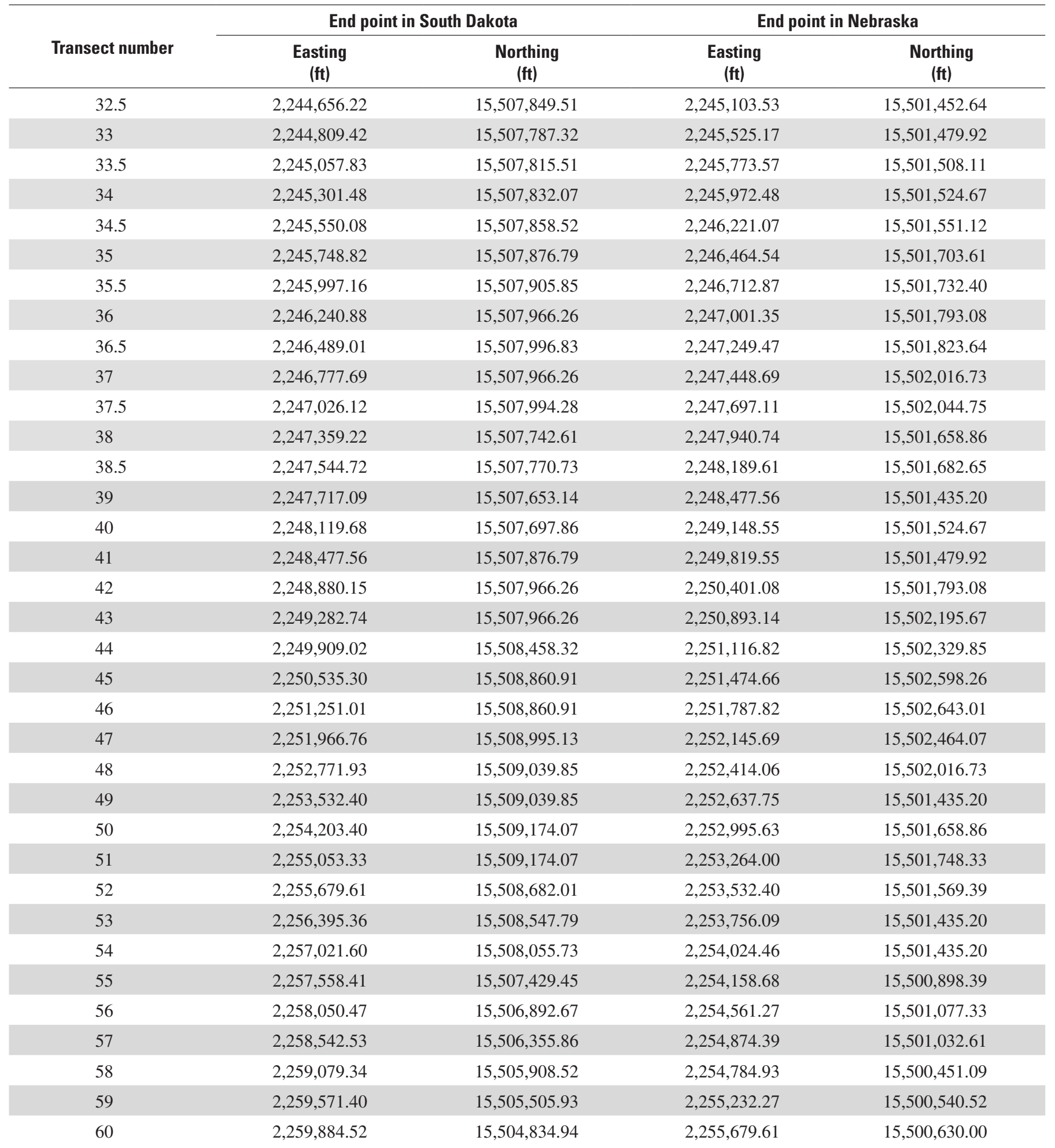


Table 2. Coordinates of transect end points, in Universal Transverse Mercator feet, Zone 14, and North American Datum of 1983.-Continued

[ft, feet; RM, river mile]

\begin{tabular}{|c|c|c|c|c|}
\hline \multirow[b]{2}{*}{ Transect number } & \multicolumn{2}{|c|}{ End point in South Dakota } & \multicolumn{2}{|c|}{ End point in Nebraska } \\
\hline & $\begin{array}{c}\text { Easting } \\
\text { (ft) }\end{array}$ & $\begin{array}{c}\text { Northing } \\
\text { (ft) }\end{array}$ & $\begin{array}{c}\text { Easting } \\
\text { (ft) }\end{array}$ & $\begin{array}{c}\text { Northing } \\
\text { (ft) }\end{array}$ \\
\hline \multicolumn{5}{|c|}{ Upstream study area (encompassing RM 769.8) } \\
\hline 1 & $2,200,344.39$ & $15,532,854.37$ & $2,202,014.17$ & $15,525,827.45$ \\
\hline 2 & $2,201,318.43$ & $15,532,923.92$ & 2,201,979.39 & $15,525,479.58$ \\
\hline 3 & $2,202,445.89$ & $15,532,923.92$ & $2,201,701.08$ & $15,525,236.08$ \\
\hline 4 & $2,203,266.49$ & $15,532,645.64$ & $2,201,735.85$ & $15,525,305.63$ \\
\hline 5 & $2,204,136.14$ & $15,532,263.00$ & $2,202,048.94$ & $15,526,140.50$ \\
\hline 6 & $2,204,658.68$ & $15,532,046.40$ & 2,202,396.09 & $15,525,887.16$ \\
\hline 7 & $2,205,128.30$ & $15,531,873.86$ & $2,202,865.74$ & $15,525,714.62$ \\
\hline 8 & $2,205,597.95$ & $15,531,701.35$ & $2,203,335.39$ & $15,525,542.11$ \\
\hline 9 & $2,206,067.60$ & $15,531,528.81$ & $2,203,805.01$ & $15,525,369.57$ \\
\hline 10 & $2,206,640.80$ & $15,531,288.95$ & $2,204,101.37$ & $15,525,236.08$ \\
\hline 11 & $2,207,241.35$ & $15,530,993.41$ & $2,204,444.11$ & $15,525,057.86$ \\
\hline 12 & 2,207,693.94 & $15,530,780.13$ & $2,204,896.71$ & $15,524,844.57$ \\
\hline 13 & $2,208,146.54$ & $15,530,597.90$ & $2,205,349.30$ & $15,524,631.29$ \\
\hline 14 & $2,208,599.13$ & $15,530,353.55$ & $2,205,801.89$ & $15,524,418.00$ \\
\hline 15 & $2,209,051.72$ & $15,530,140.27$ & $2,206,254.48$ & $15,524,204.71$ \\
\hline 16 & $2,209,319.37$ & $15,530,419.30$ & $2,206,953.89$ & $15,523,983.75$ \\
\hline 17 & $2,209,562.87$ & $15,530,210.57$ & $2,207,649.62$ & $15,523,775.02$ \\
\hline 18 & $2,209,702.04$ & $15,530,071.43$ & $2,208,470.94$ & $15,523,575.84$ \\
\hline 19 & $2,209,980.32$ & $15,530,140.99$ & $2,209,180.23$ & $15,523,427.16$ \\
\hline 20 & $2,210,307.82$ & $15,529,985.18$ & $2,209,878.85$ & $15,523,437.59$ \\
\hline 21 & $2,210,807.06$ & $15,529,952.44$ & $2,210,377.70$ & $15,523,404.85$ \\
\hline 22 & $2,211,306.31$ & $15,529,919.69$ & $2,210,876.98$ & $15,523,372.10$ \\
\hline 22.5 & $2,211,611.01$ & $15,529,902.23$ & $2,211,054.03$ & $15,523,377.54$ \\
\hline 23 & $2,211,963.16$ & $15,529,897.48$ & $2,211,163.06$ & $15,523,322.79$ \\
\hline 23.5 & $2,212,211.33$ & $15,529,867.28$ & $2,211,332.52$ & $15,523,288.03$ \\
\hline 24 & $2,212,554.53$ & $15,529,793.12$ & $2,211,545.74$ & $15,523,357.57$ \\
\hline 24.5 & $2,212,886.71$ & $15,529,745.91$ & $2,211,729.66$ & $15,523,326.13$ \\
\hline 25 & $2,213,221.43$ & $15,529,599.35$ & $2,211,913.65$ & $15,523,169.31$ \\
\hline 25.5 & $2,213,633.20$ & $15,529,546.84$ & $2,212,065.58$ & $15,523,151.95$ \\
\hline 26 & $2,214,050.36$ & $15,529,445.25$ & $2,212,171.89$ & $15,523,079.29$ \\
\hline 26.5 & $2,214,354.80$ & $15,529,360.22$ & $2,212,301.97$ & $15,523,015.10$ \\
\hline 27 & $2,214,676.54$ & $15,529,375.70$ & $2,212,415.39$ & $15,523,009.70$ \\
\hline 27.5 & $2,214,976.87$ & $15,529,298.02$ & $2,212,588.12$ & $15,522,952.89$ \\
\hline
\end{tabular}


Table 2. Coordinates of transect end points, in Universal Transverse Mercator feet, Zone 14, and North American Datum of 1983.-Continued

[ft, feet; RM, river mile]

\begin{tabular}{|c|c|c|c|c|}
\hline \multirow[b]{2}{*}{ Transect number } & \multicolumn{2}{|c|}{ End point in South Dakota } & \multicolumn{2}{|c|}{ End point in Nebraska } \\
\hline & $\begin{array}{l}\text { Easting } \\
\text { (ft) }\end{array}$ & $\begin{array}{l}\text { Northing } \\
\text { (ft) }\end{array}$ & $\begin{array}{c}\text { Easting } \\
\text { (ft) }\end{array}$ & $\begin{array}{l}\text { Northing } \\
\text { (ft) }\end{array}$ \\
\hline 28.5 & $2,215,499.41$ & $15,529,136.28$ & $2,212,960.07$ & $15,522,776.40$ \\
\hline 29 & $2,215,720.14$ & $15,529,097.39$ & $2,213,180.71$ & $15,522,522.70$ \\
\hline 30 & $2,216,172.37$ & $15,528,923.47$ & $2,213,702.50$ & $15,522,174.83$ \\
\hline 30.5 & $2,216,357.87$ & $15,528,850.12$ & $2,214,006.44$ & $15,522,057.11$ \\
\hline 31 & $2,216,520.24$ & $15,528,784.30$ & $2,214,328.68$ & $15,521,931.32$ \\
\hline 31.5 & $2,216,693.79$ & $15,528,713.27$ & $2,214,628.51$ & $15,521,845.60$ \\
\hline 32 & $2,216,868.11$ & $15,528,679.97$ & $2,214,954.82$ & $15,521,826.96$ \\
\hline 33.5 & $2,217,328.30$ & $15,528,563.97$ & $2,215,710.93$ & $15,521,534.57$ \\
\hline 34 & $2,217,529.06$ & $15,528,436.43$ & $2,216,033.23$ & $15,521,618.23$ \\
\hline 34.5 & $2,217,676.66$ & $15,528,389.79$ & $2,216,407.64$ & $15,521,609.22$ \\
\hline 35 & $2,217,772.57$ & $15,528,332.07$ & $2,216,833.30$ & $15,521,687.82$ \\
\hline 36 & $2,218,155.21$ & $15,528,262.51$ & $2,217,389.89$ & $15,521,826.96$ \\
\hline 37 & $2,218,477.58$ & $15,528,200.67$ & $2,218,108.49$ & $15,521,649.37$ \\
\hline 38 & $2,219,824.96$ & $15,527,775.51$ & $2,217,007.25$ & $15,520,226.77$ \\
\hline 39 & $2,221,007.73$ & $15,526,836.27$ & $2,216,311.51$ & $15,520,400.72$ \\
\hline 40 & $2,221,842.60$ & $15,525,827.45$ & $2,216,137.56$ & $15,521,061.64$ \\
\hline 46 & $2,223,208.48$ & $15,523,995.43$ & $2,218,986.77$ & $15,518,972.21$ \\
\hline 47 & $2,223,444.04$ & $15,523,809.41$ & $2,219,536.41$ & $15,518,538.19$ \\
\hline 48 & $2,223,690.50$ & $15,523,638.11$ & $2,220,111.47$ & $15,518,138.45$ \\
\hline 49 & $2,223,946.93$ & $15,523,482.14$ & $2,220,709.86$ & $15,517,774.54$ \\
\hline 50 & $2,224,212.42$ & $15,523,342.08$ & $2,221,329.32$ & $15,517,447.77$ \\
\hline 51 & $2,224,418.49$ & $15,523,218.49$ & $2,221,967.51$ & $15,517,159.35$ \\
\hline 52 & $2,224,766.48$ & $15,523,111.80$ & $2,222,622.13$ & $15,516,910.40$ \\
\hline 53 & $2,225,053.00$ & $15,523,022.40$ & $2,223,290.70$ & $15,516,701.84$ \\
\hline 54 & $2,225,344.47$ & $15,522,950.65$ & $2,223,970.72$ & $15,516,534.39$ \\
\hline 55 & $2,225,639.74$ & $15,522,896.81$ & $2,224,659.72$ & $15,516,408.73$ \\
\hline
\end{tabular}


Data collected along each transect included (1) water depth (bathymetry) in areas with depths generally greater than $3 \mathrm{ft}$; and (2) land-surface elevation (topographic) on the high bank and shore and in sandbar areas and riverbed elevation in areas with water depths generally less than $3 \mathrm{ft}$. In addition, water-velocity data were collected on selected transects in the upstream study area. Water-surface elevation data also were collected at many points throughout both study areas. Specific information regarding instruments used, data-collection methods, and quality-assurance/quality-control (QA/QC) measures are described in the following sections.

The hydrographic surveys consisted of bathymetric and topographic surveys along each transect. The transects were bathymetrically and/or topographically surveyed from high bank to high bank using global positioning system (GPS) guidance to remain on the pre-determined transect line. The topographic surveys were conducted where bathymetric data collection was not possible. Transects generally were perpendicular to the direction of flow. Data acquisition, processing, accuracy, and QA/QC conformed to the recommended standards in "Other General Surveys and Studies" (U.S. Army Corps of Engineers, 2002, table 3-1, p. 3-1) and USGS guidelines.

\section{Bathymetric Survey}

Bathymetric data were collected by navigating a survey vessel along the data-collection transects. Navigational maps of the two study areas were developed by creating a georeferenced drawing interface file (DXF file) from digital orthophoto quadrangles (DOQs) of the study areas. The DXF files for approximate high bank and midchannel locations (fig. 1) were imported into Hypack hydrographic software (Coastal Oceanographics, 2006) where channel design and boundaries were used to create border files (.BRD) and planned line files (.LNW) used for data-collection activities. The planned line system used a cross-section spacing of $500 \mathrm{ft}$, generally oriented perpendicular to channel flow, extending roughly $2 \mathrm{mi}$ upstream and $2 \mathrm{mi}$ downstream from each construction area. Individual cross sections extended from high bank to high bank across the river channel. Prior to postconstruction data collection, additional transects were added between original transects in selected parts of the study areas.

\section{Instrumentation}

All instrumentation and equipment used during this project met the requirements noted in "Engineering and Design - Hydrographic Surveying” (U.S. Army Corps of Engineers, 2002; chapter 9). Streambed geometry data were collected using a survey grade Innerspace Technology 456 echosounder coupled with a 200-kilohertz ( $\mathrm{kHz})$ 3 -degree beam transducer. The transducer was mounted off the starboard side of a 14-ft USGS boat. A 12-channel differentially corrected Trimble Ag132 GPS (DGPS) was used for horizontal positioning for the bathymetric and watervelocity data sets collected by boat. The DGPS antenna was mounted directly above the transducer (fig. 4). An Innerspace Technology 443A Velocity Profiler was used to measure and record speed-of-sound profiles in the water column. Speedof-sound profiles for the water column then were used to calibrate the echosounder. All digital bathymetric data were logged into a ruggedized field laptop computer running Hypack hydrographic software.

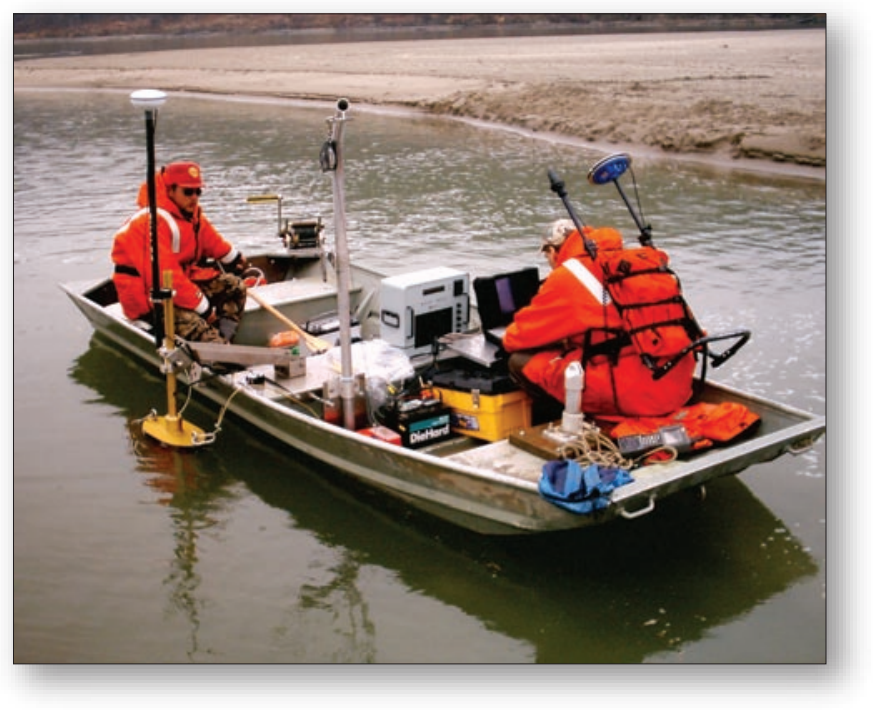

Figure 4. Configuration of instruments used in bathymetric and water-velocity data collection.

\section{Methods}

Equipment setup, calibration, and data-collection procedures complied with the guidelines described by the USACE (2002, chapter 9). To map the streambed geometry of the study area, a continuous series of acoustic pulses were emitted from the bathymetric transducer. The pulses traveled through the water column, reflected off of the streambed, and returned to the transducer. A time-of-travel equation (U.S. Army Corps of Engineers, 2002, chapter 9) was applied to the returning acoustical pulse to determine water depth and streambed geometry. Because bathymetric data collection on rough water was avoided, errors associated with pitch and roll of the survey vessel were not corrected. Digital bathymetric data and horizontal positioning were logged concurrently on a computer running Hypack software. Digital data were processed within Hypack to remove erroneous readings in the data set. Instrument draft and water depth at each horizontal location were then subtracted from a water-surface elevation determined using real-time kinematic (RTK) GPS to calculate streambed elevation. 


\section{Quality Assurance/Quality Control}

Data accuracies for the bathymetric survey satisfied the requirements set forth in "Other General Surveys and Studies" (U.S. Army Corps of Engineers, 2002, table 3-1, p. 3-1). Equipment setup and calibration required an initial measurement of the transducer draft, a speed-of-sound velocity profile, and a bar check (fig. 5). Approximately 10 percent of the cross sections were re-run with a different deployment of the equipment. This duplicate data allowed an assessment of repeatability of both horizontal location and elevation to assure the quality of the data. In addition, the bathymetric analog data were captured and downloaded for future referencing.

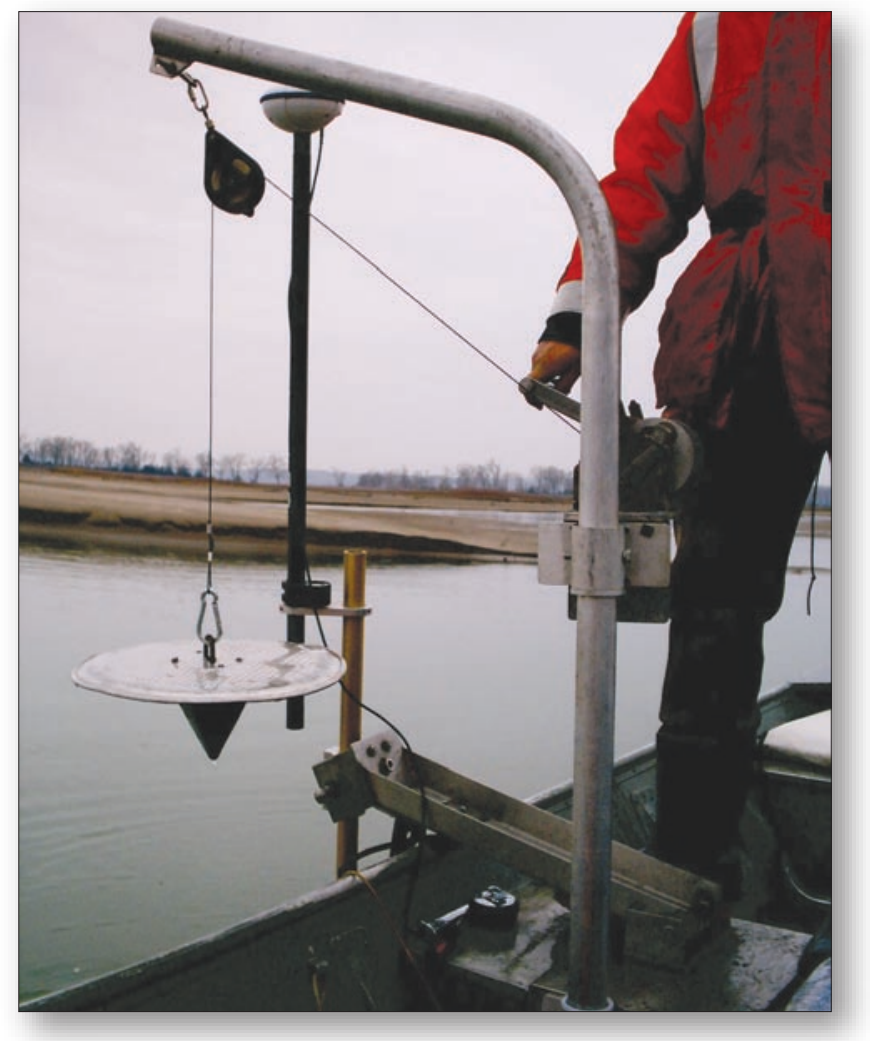

Figure 5. Apparatus used to conduct bar check of echosounder transducer.

\section{Topographic Survey}

GPS instruments utilize satellite signals to determine the position of a point on the earth. For the topographic survey, GPS instruments were used to collect land-surface elevation data on the dry-land parts of the transects and stream-bottom elevation data on areas of the transects too shallow for the bathymetric vessel. The GPS data then were combined with the bathymetric data to form cross sections along each transect. GPS instruments also were used to record watersurface elevations throughout the study area and for horizontal location determination of water-velocity data points.

\section{Instrumentation}

GPS instruments used to survey shallow water habitat and banks of the study areas on the Missouri River near RM 761.3 and RM 769.8 included Ashtech Z-Xtreme receivers and Geodetic IV revision A antennae. The Geodetic IV revision A GPS antenna is a dual-frequency (L1/L2) model. The horizontal accuracy of the Z-Xtreme was explained in the reference manual as 5 millimeters $(\mathrm{mm})+1$ part per million $(\mathrm{ppm})$ when collection type is set to static and the data are post-processed. The accuracy was 1.6 centimeters $(\mathrm{cm})+2 \mathrm{ppm}$ for RTK points with five satellites and position dilution of precision (PDOP) less than 4 (Ashtech Precision Products, 2001).

To minimize distance-related errors, base stations were established near the area to be surveyed rather than relying on benchmarks distant from the site. Most established bases were set using static GPS surveying procedures and post-processed against three first-order vertical and high-order horizontal National Geodetic Survey (NGS) benchmarks. The accuracy for these bases was determined by the accuracy of the NGS benchmark plus the quality of the data collected. Table 3 shows the NGS benchmark relative accuracy and an estimated spatial accuracy (Doyle and McKay, 2000). Post-processing a network with a time span of at least 2 hours usually produced positions with a 95-percent error of less than 0.02 meter (m) horizontal and vertical. If time did not allow for NGS benchmarks to be located, static sessions were processed through the NGS Online Positioning Users Service (OPUS, at $h t t p: / / w w w$. ngs.noaa.gov/OPUS/), which processed the session against three continuously operating reference stations. A 2- or 3-hour session processed through OPUS usually produced a position with a peak-to-peak error of less than 0.02-m horizontal and less than $0.03-\mathrm{m}$ vertical.

Table 3. National Geodetic Survey benchmark relative accuracy and estimated spatial accuracy.

$[\mathrm{cm}$, centimeters]

\begin{tabular}{ccc}
\hline Order & Relative accuracy & $\begin{array}{c}\text { Estimated spatial } \\
\text { accuracy }\end{array}$ \\
\hline A & $1: 10,000,000$ & $3 \mathrm{~cm}$ \\
B & $1: 1,000,000$ & $5 \mathrm{~cm}$ \\
1 & $1: 100,000$ & $10 \mathrm{~cm}$ \\
2 & $1: 50,000$ & $20 \mathrm{~cm}$ \\
3 & $1: 10,000$ & $50 \mathrm{~cm}$ \\
\hline
\end{tabular}


Thus, accuracy in each RTK point was the accuracy of the established base plus $1.6 \mathrm{~cm}+2 \mathrm{ppm}$. During surveys of the ESH sites, RTK data usually were collected within $6 \mathrm{mi}$ of the base station, so each RTK point had an approximate horizontal error of $3 \mathrm{~cm}$ and a vertical error of $10 \mathrm{~cm}$ when collected by backpack mount (fig. 6).

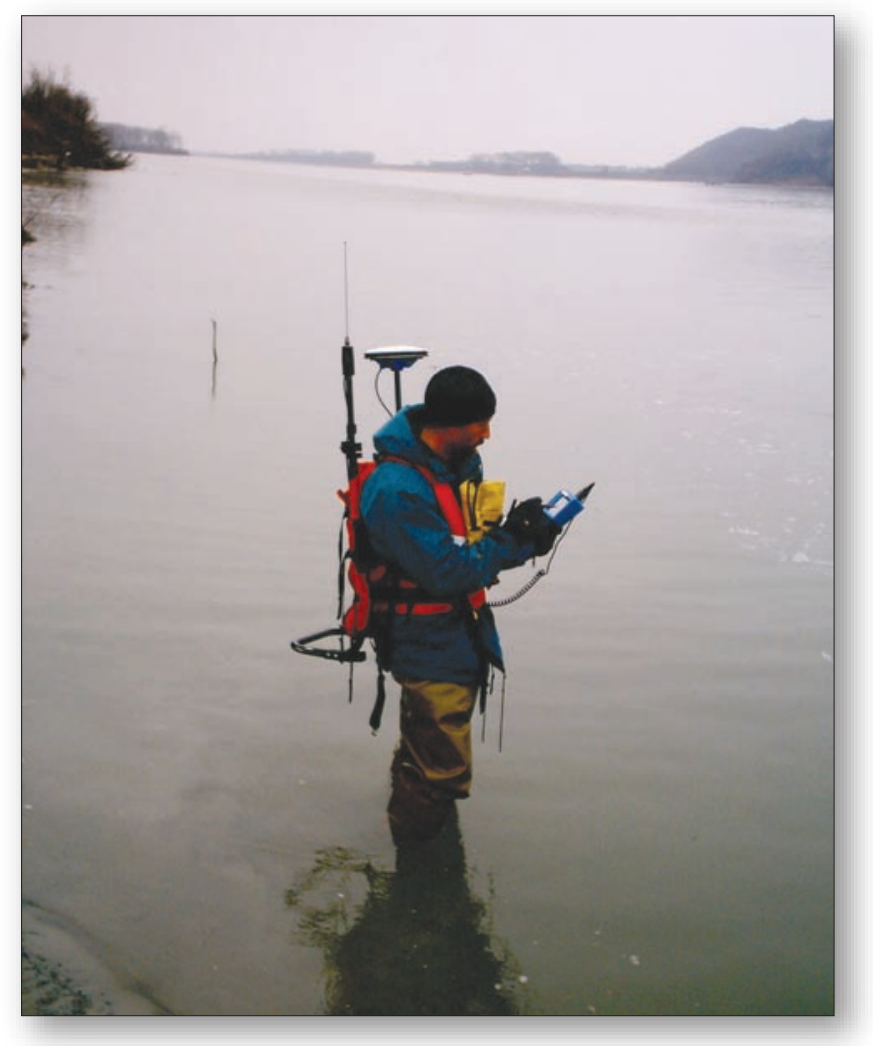

Figure 6. Configuration of real-time kinematic global positioning system using backpack mount.

\section{Methods}

For the topographic survey, the surveyed transects were set up using DOQs in Hypack software as described previously in the Bathymetric Survey section. The spacing was established by the USACE and modified between pre- and postconstruction data collection. The preconstruction transects were spaced uniformly at $500 \mathrm{ft}$. For postconstruction data collection, transects were added in the construction areas between the existing transects. The spacing was reduced to $250 \mathrm{ft}$ at the center, then remained at $500 \mathrm{ft}$, then increased to $1,000 \mathrm{ft}$ and $1,500 \mathrm{ft}$ at the ends of each reach. The transects were set up perpendicular to the channel, usually along the thalweg (figs. 2 and 3).
Elevation measurements were collected using RTK GPS with a backpack mount (fig. 6). Transects were traversed to measure overbank, shore/bank, sandbar, and riverbed elevations for shallow parts of the cross sections not able to be navigated by boat (sections that were typically $3 \mathrm{ft}$ deep or less). Horizontal distances between elevation measurements along the transects generally were less than $2 \mathrm{ft}$. Horizontal deviation did not exceed $10 \mathrm{ft}$ on either side of a given transect. GPS measurements were only recorded when the error associated with each point was within $0.03 \mathrm{~m}$ horizontal and $0.05 \mathrm{~m}$ vertical.

\section{Quality Assurance/Quality Control}

QA/QC activities for the topographic survey were conducted on a daily basis. Accuracy of the RTK GPS horizontal-position and elevation data was assessed by having each surveyor occupy (stand with the GPS antenna approximately over) points of known horizontal position and elevation a minimum of two times per day (before and after data-collection activities). For example, the standard deviations of the northing and easting and the elevation of 40 occupations at the upstream checkpoint were $0.30,0.47$, and $0.27 \mathrm{ft}$, respectively. The standard deviations of the northing and easting and the elevation of 32 occupations at the downstream checkpoint was $0.44,0.35$, and $0.26 \mathrm{ft}$, respectively. Approximately 10 percent of the transects were surveyed in duplicate to provide additional QA/QC information to verify the accuracy and precision of datacollection activities.

\section{Water-Velocity Data Collection}

Water-velocity data were collected along selected transects of the upstream study area (RM 769.8) using two methods. In deep water (generally greater than $3 \mathrm{ft}$ ), watervelocity profile data were collected using an Acoustic Doppler Current Profiler (ADCP) tethered to the survey vessel. In shallow water (generally less than $3 \mathrm{ft}$ ), point water-velocity data were collected using a handheld Acoustic Doppler Velocimeter (ADV).

\section{Instrumentation}

The ADCP used to collect water-velocity profiles was a 1200-kHz Teledyne Rowe Deines Instruments (RDI) Rio Grande. The ADCP was mounted directly in front of the bathymetric transducer, off the starboard side near the bow of the survey vessel. ADCP data were logged concurrently with the bathymetric data into a separate ruggedized field laptop computer running WinRiver software (RD Instruments, 2003). The ADCP derived its horizontal location from the same DGPS antenna as the bathymetric transducer. 
The handheld ADV used to collect point water-velocity data was a SonTek/Yellow Springs Instruments (YSI) FlowTracker. The ADV was mounted on a top-setting wading rod and used to measure the velocity at a point six-tenths of the water depth. Horizontal positioning of the FlowTracker was recorded with either a Total Station or RTK GPS.

\section{Methods}

ADCP water-velocity profiles were obtained on 47 predetermined transects at the upstream study area (RM 769.8) only. Because of instrument limitations and vessel draft, the ADCP was used only in depths greater than approximately $3 \mathrm{ft}$. Equipment setup, calibration, and data-collection procedures complied with the guidelines described by Oberg and others (2005). Velocity data were collected using Mode 12 with a $10-\mathrm{cm}$ bin cell size and a blanking distance of 20-25 cm. Multiple bins arranged in a vertical column or "ensemble" occurred every 1.45 seconds. Boat speed was maintained at or near 2.0 knots. Data acquisition based on boat speed and DGPS coverage occurred approximately every $5 \mathrm{ft}$. Velocity and DGPS data were logged on a separate computer running WinRiver ADCP software.

FlowTrackers were used to collect point water-velocity data in parts of the selected transects that were too shallow for the ADCP (less than approximately $3 \mathrm{ft}$ ). Point water-velocity data were collected approximately every $50 \mathrm{ft}$ along the transect and included a velocity and a compass direction of flow. The average velocity measured over a 40 -second time interval was recorded. Horizontal positioning of the point velocity data was accomplished with a Total Station set up on the transect or by utilizing RTK GPS. Total Station data and FlowTracker data were later combined by point name. When RTK GPS was used for positioning, the velocity and compass direction were used as the point description stored in the GPS.

\section{Quality Assurance/Quality Control}

The ADCP was calibrated before data collection on a daily basis and consisted of a measurement of equipment draft, an internal diagnostics test, and a compass calibration. Each FlowTracker was calibrated daily before data collection by submersing in water and running an internal diagnostics test.

\section{Bathymetric, Topographic, and Water- Velocity Data}

Raw data collected as described previously were processed and corrected, where applicable. The resulting data sets were used to generate GIS shapefiles, which then were used to develop the maps and cross sections that help visualize the data. The specific processes used are described in the following sections.

\section{Bathymetric and Topographic Data}

The processed bathymetric data were output in an XYZ format, where $\mathrm{X}$ and $\mathrm{Y}$ are the horizontal locations of a specific point and $\mathrm{Z}$ is the riverbed elevation at that point. The XYZ file was used to generate a geospatial data set, and GIS software programs were used to change the data to a different projection and/or visualize the data. Although vertical data were collected in the North American Vertical Datum of 1988, they are presented here in the National Geodetic Vertical Datum of 1929 to facilitate comparison with historical USACE and USGS data. Topographic data collected with GPS were output in a similar format although each point with an XYZ location also has a label to provide an abbreviated description of the point. For example, a label of LEW denotes left edge of water, and TRB denotes top of right bank. A more complete list of label descriptors is found in the metadata associated with the GIS shapefiles.

\section{Transect Cross Sections}

For data visualization purposes, a series of cross-section plots were generated for each transect. These plots are located in Supplemental Information Section A at the end of this report. ArcMap software (Harlow and others, 2000) was used to select from the geospatial bathymetric and topographic data sets all data points that were within $10 \mathrm{ft}$ of a transect. The resulting data points were output into an XYZ text file and sorted by transect number, northing, and point identifier. The sorted file was imported into a database using Geosoft's Oasis Montaj version 6.3.0 (Geosoft, 2006) and organized by transect number. In Oasis Montaj, the projection was defined as UTM Zone 14, NAD 83, feet, and data were once again sorted by northing and easting. A non-linear filter (despike) was applied using nlfilt.gx with a width of 1 (fiducials) and a tolerance of 1 . This defines the magnitude of the filter as $1 \mathrm{ft}$. The filtered database then was loaded into Encom's Profile Analyst version 6.00 (Rockware, 2006) (expert-user forced entry), where curve profiles were generated.

Because of various field conditions encountered during data collection, small data gaps occasionally are present in cross sections. Every effort was made to navigate the bathymetric vessel such that the data would be collected within $10 \mathrm{ft}$ of the transect. Most of the time, this was achieved, but swift currents, wind, and obstacles sometimes made this impossible. Submerged timber, beaver dams, and log jams occasionally prevented the bathymetric vessel from staying within $10 \mathrm{ft}$ of the transect. Similarly, steep or unstable banks sometimes prevented collection of RTK GPS data every $2 \mathrm{ft}$. Log jams and beaver dams in shallow water also occasionally affected GPS data collection. In high bank and island areas, thick tree and 
brush cover sometimes made it difficult to stay on the transect. Some of the same areas had a vegetative canopy sufficiently thick to interfere with satellite reception. In such areas, it was difficult to maintain continuous lock on the satellites, and occasionally the signal was blocked completely. Because the areas with thick canopy were always on the bank or on the larger islands, they were generally at or above the high-bank elevation.

\section{Surface Visualization Maps}

A color-shaded relief map was generated for the postconstruction land surface from transect 23 to 39 at the downstream study area near RM 761.3 (fig. 7) and from transect 22 to 35 at the upstream study area near RM 769.8 (fig. 8). Comparisons of the hydrographic data collected in 2004 before ESH construction and in 2005 and 2006 following ESH construction were made to document changes in channel and overbank geometry. This was accomplished by subtracting the postconstruction surface from the preconstruction surface. The resulting maps show the elevation difference between preand postconstruction surfaces at the downstream (fig. 9) and upstream (fig. 10) study areas.

\section{Water-Velocity Data}

ADCP water-velocity data collected in WinRiver software were output in an XYZ comma-delimited file format.
The data then were sorted in Microsoft Excel by transect number and northing, and easting and saved as a new file. The sorted file was imported into a database using Geosoft's Oasis Montaj version 6.3.0 (Geosoft, 2006) and organized by transect number. Depth (D1) and velocity (R1) fields were loaded as arrays. In Oasis Montaj, the projection was defined as UTM Zone 14, NAD 83, feet, and data once again were sorted by northing and easting. An average velocity channel (column) was generated from the velocity array (R1), and the final database was saved.

The final database was loaded into Encom's Profile Analyst version 6.00 (Rockware, 2006), where watervelocity profiles were generated as section plots (by D1 and R1). A data map was generated using the Look Up Table (LUT) editor, displaying values at 0 as transparent and a linear gradation of colors between -0.5 and 4.0 feet per second (ft/s). Color-shaded velocity cross sections are presented in Supplemental Information Section B for all transects with water-velocity data.

Point water-velocity data from the handheld ADV units were pooled from the two collection sources (GPS and Total Station) and used to generate a GIS shapefile. Because the water velocity was already a vertical average for the depth of flow (as opposed to the vertical profile of velocities collected by the ADCP), point water-velocity data can be visualized by color-shaded points on a plan view (fig. 11). 


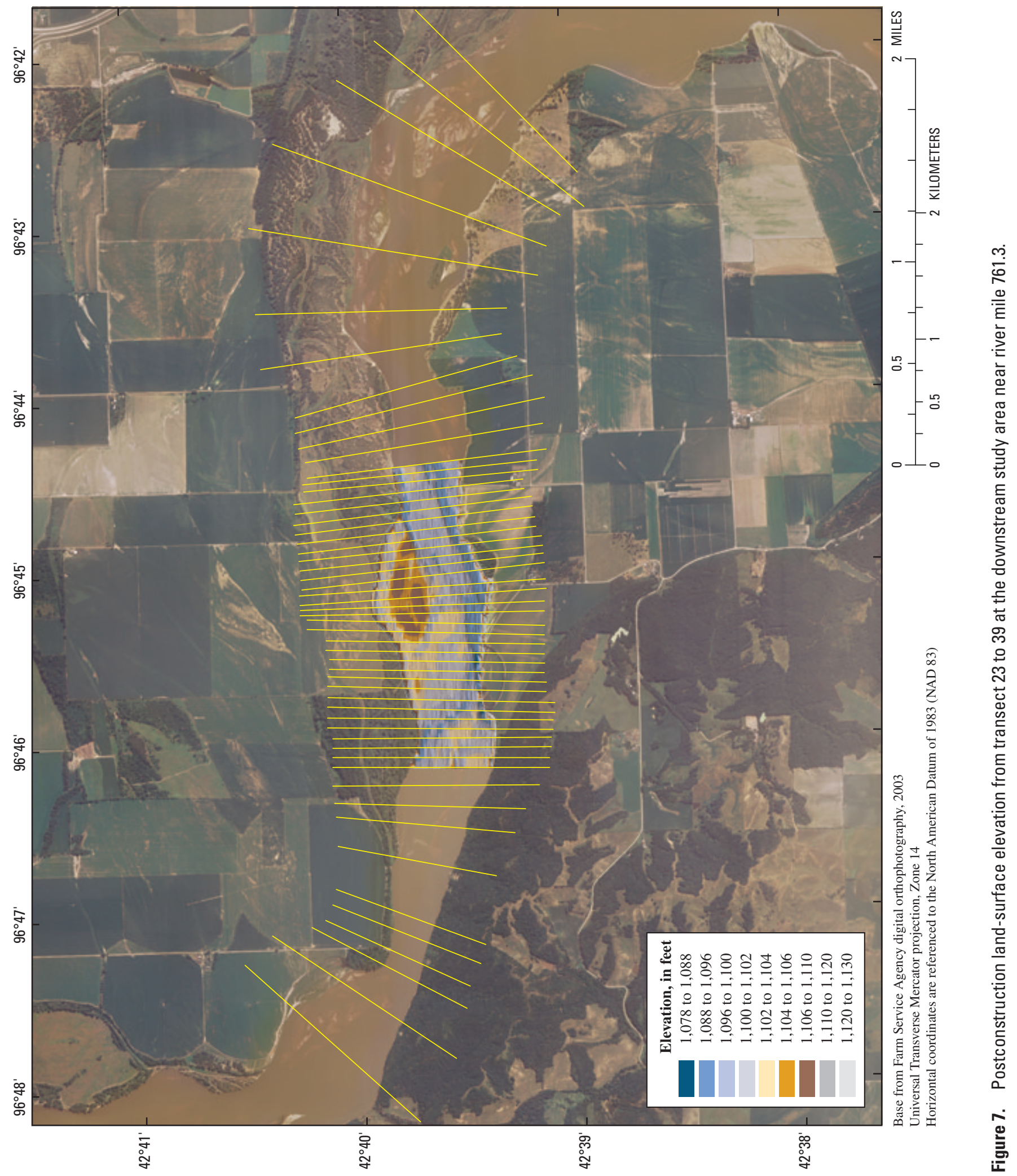




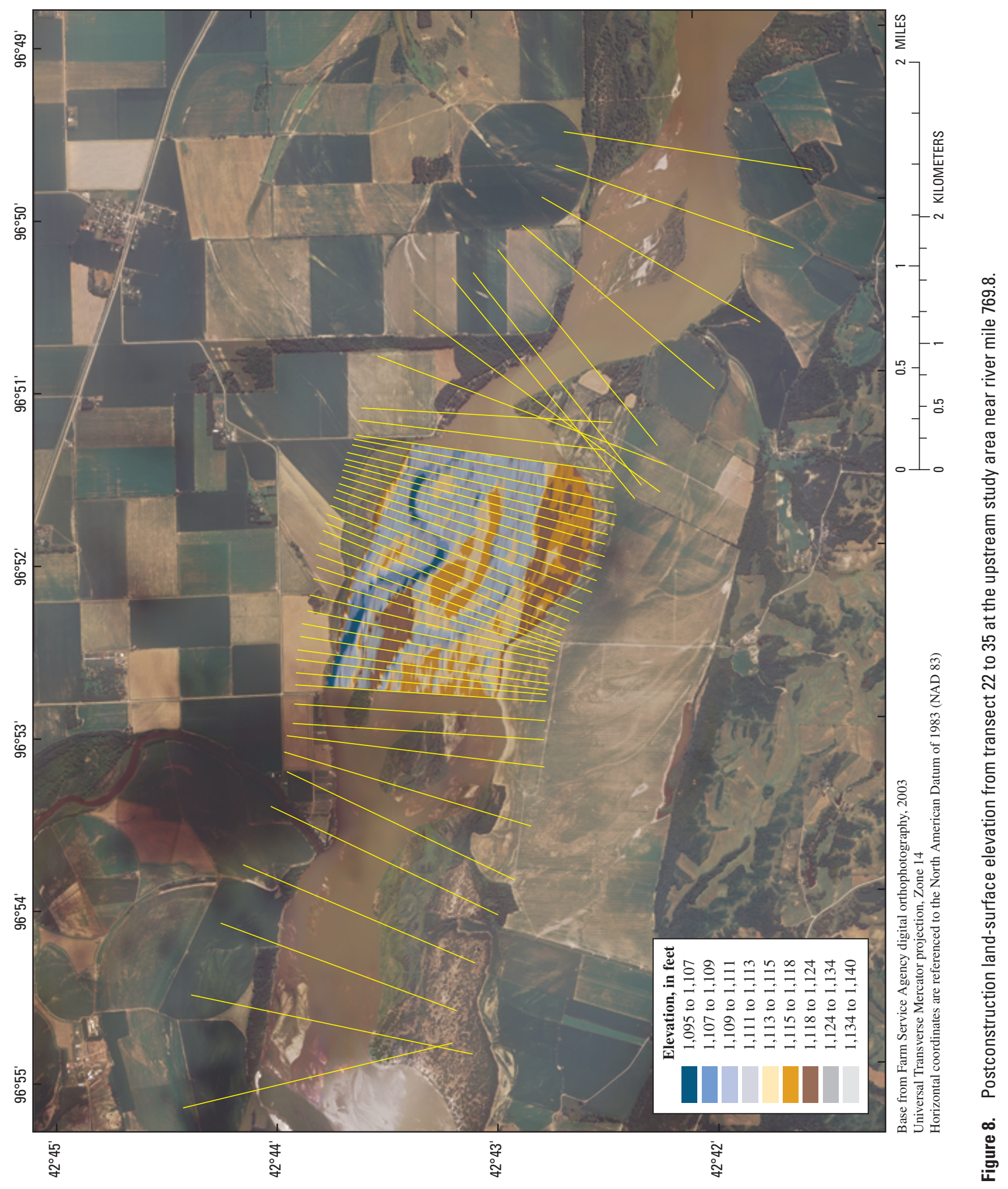




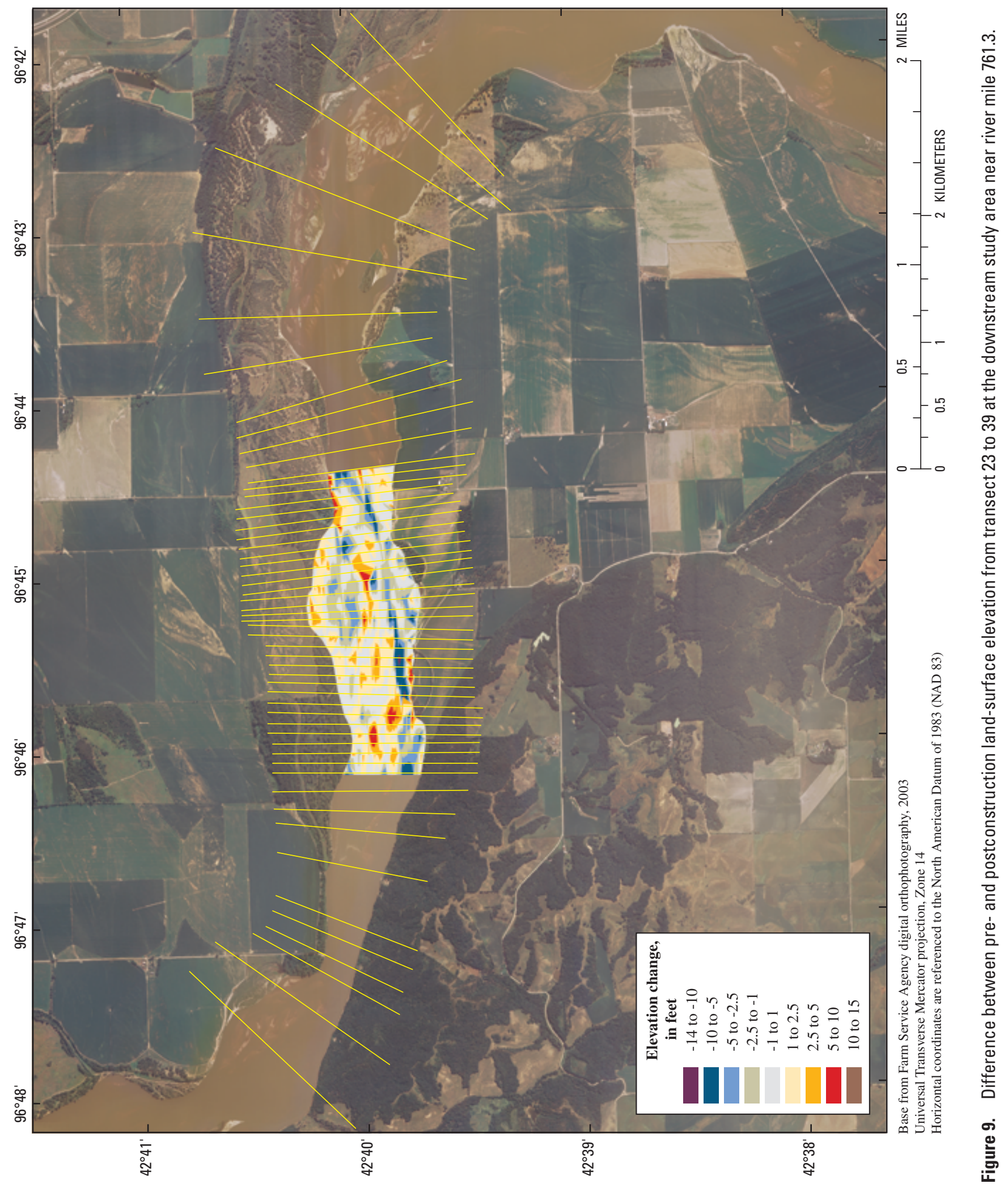




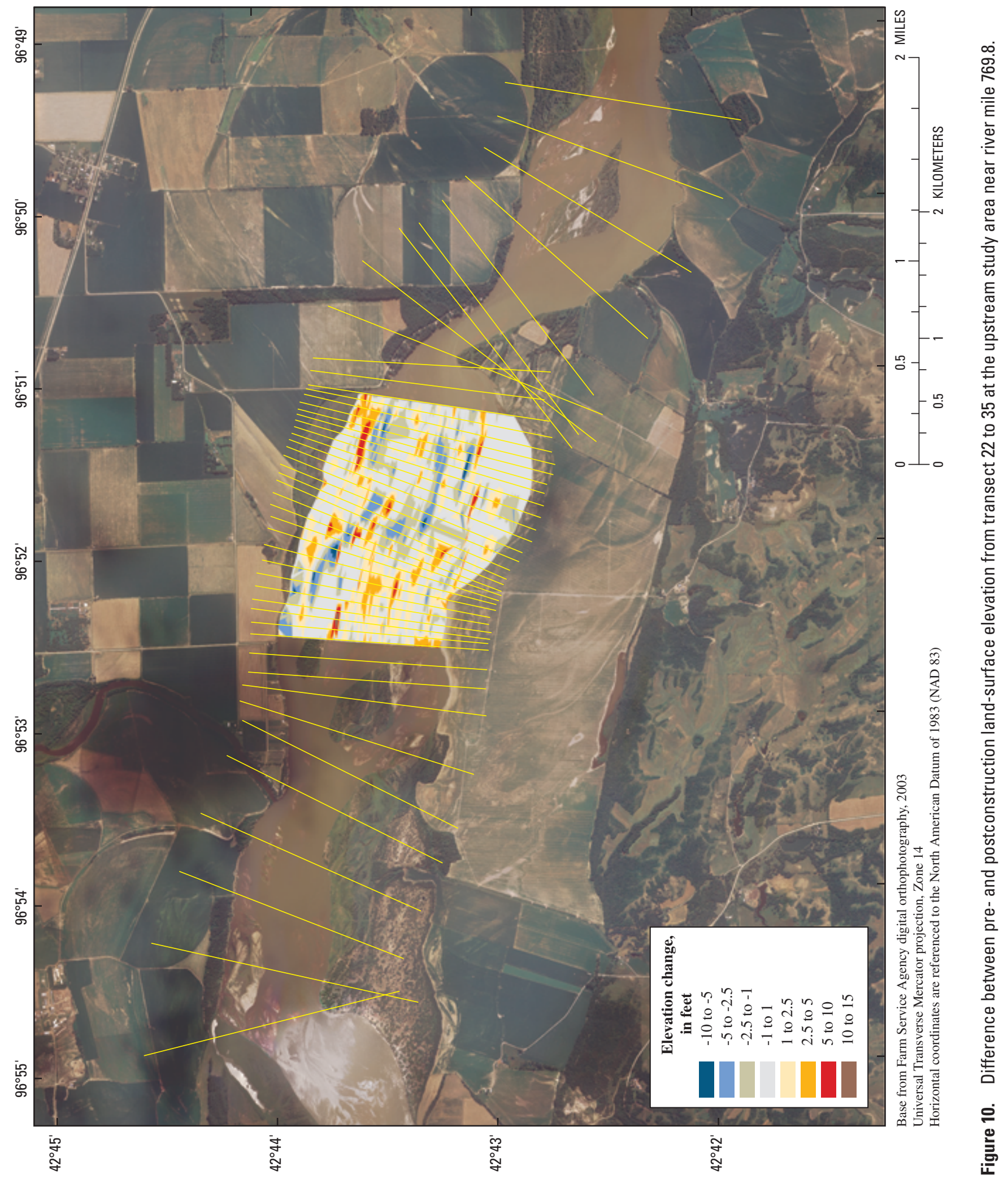




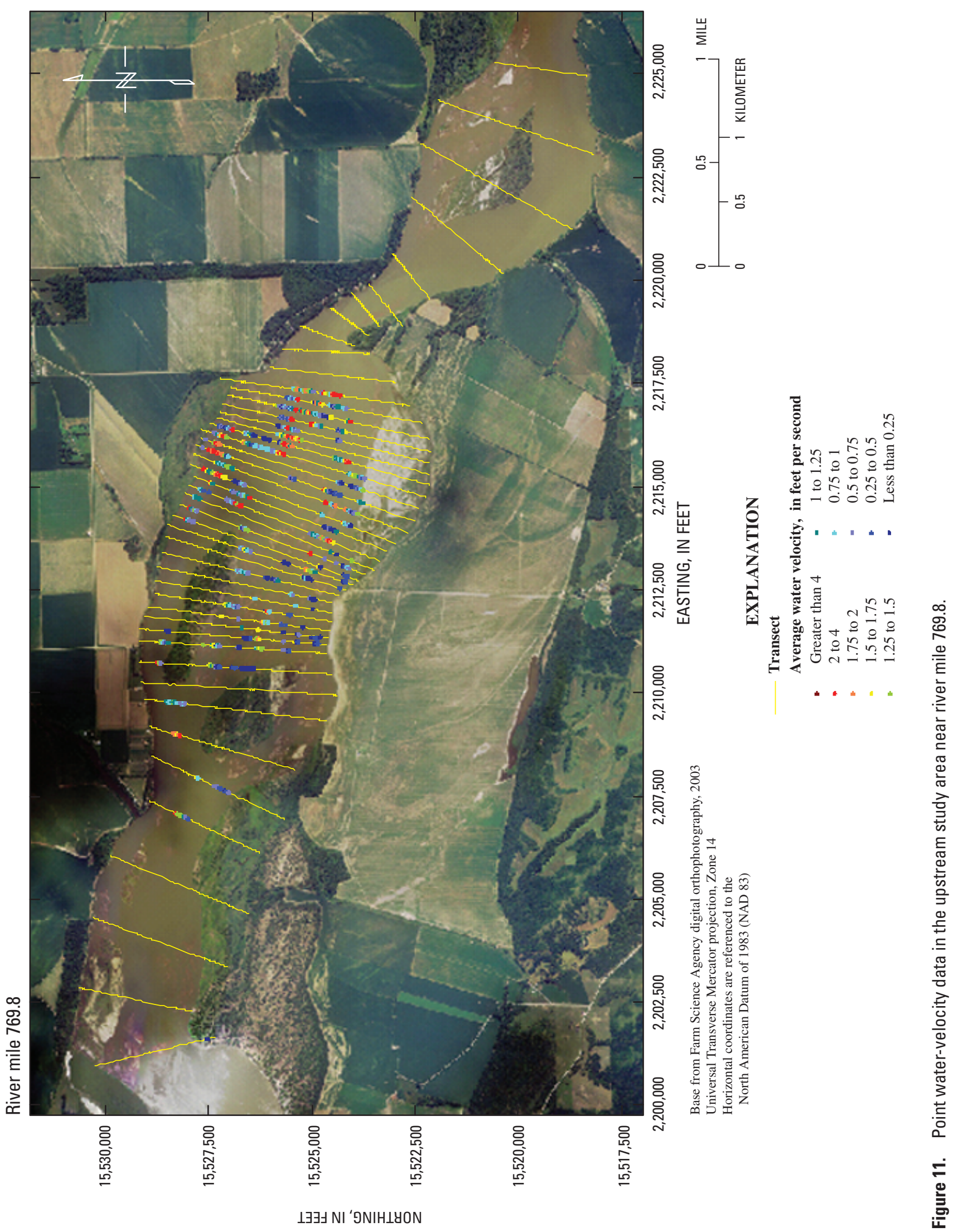




\section{Summary}

The U.S. Army Corps of Engineers (USACE) authorized a project to alter part of the Missouri River channel downstream from Gavins Point Dam in South Dakota and Nebraska. Channel alterations involved construction of emergent sandbar habitat (ESH) for endangered least terns and threatened piping plovers, as outlined in the 2000 Biological Opinion and the amended 2003 Biological Opinion (2003 BiOp). The 2003 Biological Opinion also mandates an intensive program to monitor and evaluate the success of habitat management techniques used by the USACE to meet specific habitat goals. Therefore, to assess the physical and ecological effects of the channel alterations, accurate information was required to define the Missouri River channel geometry prior to and after construction of the project. Two areas were selected by the USACE for creation and enhancement of ESH. The U.S. Geological Survey, in cooperation with the USACE, collected data to document the pre- and postconstruction channel geometry within the two areas. This report describes the methods used in data collection and presents channelgeometry (bathymetric and topographic) and water-velocity data.

The areas selected for creation and enhancement of ESH are located near river mile 761.3 and river mile 769.8 near the South Dakota towns of Elk Point and Vermillion, respectively. The USACE specified that for each ESH site, preconstruction channel-geometry information was required for a reach extending from 2 miles upstream to 2 miles downstream from the site boundaries. Thus, each of the study areas is composed of a construction area and the 2-mile upstream and down- stream buffers adjacent to the construction area. Data were collected approximately every 2 feet along the length of each transect from high bank to high bank and deviated no more than 10 feet on either side of the transect. Data collected along each transect included (1) water depths in areas with depth generally greater than 3 feet; and (2) land-surface elevation on the high bank and shore and sandbar areas and riverbed elevation in areas with water depths generally less than 3 feet. In addition, water-velocity data were collected on selected transects in the upstream study area.

Bathymetric data were collected by navigating a survey vessel along the data-collection transects. Navigational maps of the two study areas were developed by creating a georeferenced drawing interface file from digital orthophoto quadrangles of the study areas. Global positioning system instruments were used to collect land-surface elevation data on the dry-land parts of the transects and stream-bottom elevation data on areas of the transects too shallow for the bathymetric vessel. The global positioning system data then were combined with the bathymetric data to form cross sections along each transect. Water-velocity data were collected on selected transects of the upstream study area (river mile 769.8) using two methods. In deep water (generally greater than 3 feet), water-velocity profile data were collected using an Acoustic Doppler Current Profiler tethered to the survey vessel. In shallow water (generally less than 3 feet), point water-velocity data were collected using a handheld Acoustic Doppler Velocimeter. Raw data were processed and corrected, where applicable, and the resulting data sets were used to generate geographic information system shapefiles, which then were used to develop maps and cross sections. 


\section{References Cited}

Ashtech Precision Products, 2001, Operation and Reference Manual Z-Xtreme: Magellan Corporation, Santa Clara, Calif., $54 \mathrm{p}$.

Coastal Oceanographics, 2006, Hypack user's manual: Middletown, Conn., accessed October 23, 2006, at ftp://ftp.hypack.com/Documents/English/ HYPACK\%20Manual/PDF/HYpack.pdf

Doyle, D., and McKay, E., 2000, NGS survey marker accuracy: accessed March 14, 2006, at http://gpsinformation.net/main/ngs-accuracy.html

Geosoft, 2006, Geosoft Oasis Montaj product description: accessed October 23, 2006, at http://www.geosoft.com/ pinfo/oasismontaj/index.asp

Harlow, Melanie; Pfaff, Rhonda; Minami, Michael; Hatakeyama, Alan; Mitchell, Andy; Booth, Bob; Payne, Bruce; Eicher, Cory; Blades, Eleanor; Sims, Ian; Baily, Jonathan; Brennan, Pat; Stephens, Sandy; and Woo, Simon, 2000, ArcGIS 9 using ArcMap: Redlands, Calif., Environmental Systems Resources, Incorporated, 585 p.

Oberg, K.A., Morlock, S.E., and Caldwell, S.W., 2005, Quality-assurance plan for discharge measurements using acoustic doppler current profilers: U.S. Geological Survey Scientific Investigations Report 2005-5183, 435 p.
RD Instruments, 2003, WinRiver Application, Version 10.06: San Diego, Calif.

Rockware, 2006, Encom Profile Analyst product description: accessed October 23, 2006, at http://www.rockware.com/ catalog/pages/profioleanalyst.html

U.S. Army Corps of Engineers, 2002, Engineering and design - hydrographic surveying: U.S. Army Corps of Engineers Manual No. 1110-2-1003, 579 p.

U.S. Fish and Wildlife Service, 2000, Biological opinion on the operation of the Missouri River main stem reservoir system, operation and maintenance of the Missouri River Bank Stabilization and Navigation Project, and operation of the Kansas River reservoir system: Minneapolis, Minn., U.S. Fish and Wildlife Service, 286 p.

U.S. Fish and Wildlife Service, 2003, U.S. Fish and Wildlife Service 2003 amendment to the 2000 biological opinion on the operation of the Missouri River main stem reservoir system, operation and maintenance of the Missouri River Bank Stabilization and Navigation Project, and operation of the Kansas River reservoir system: Minneapolis, Minn., U.S. Fish and Wildlife Service, 298 p. 
This page intentionally left blank. 
Supplemental Information 
This page intentionally left blank. 


\section{Section A. Transect Cross Sections}

Cross sections showing pre- and postconstruction land-surface elevation for each transect are presented for the downstream (figs. A1-A54) and upstream (figs. A55-A102) study areas. Each figure contains an overhead view showing the location of a highlighted transect and the cross section for that transect. 

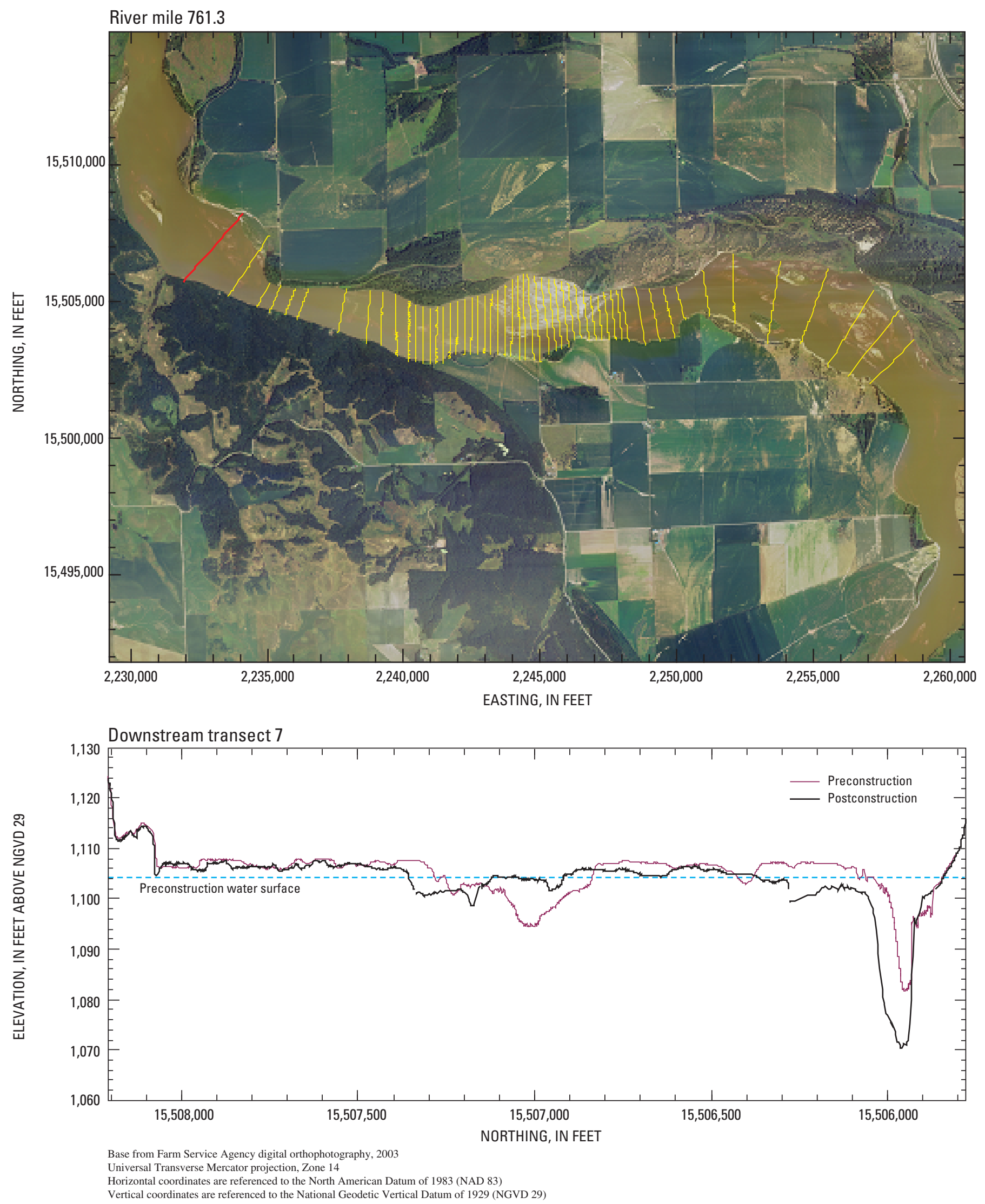

Figure A1. Location and cross section for downstream transect 7. 

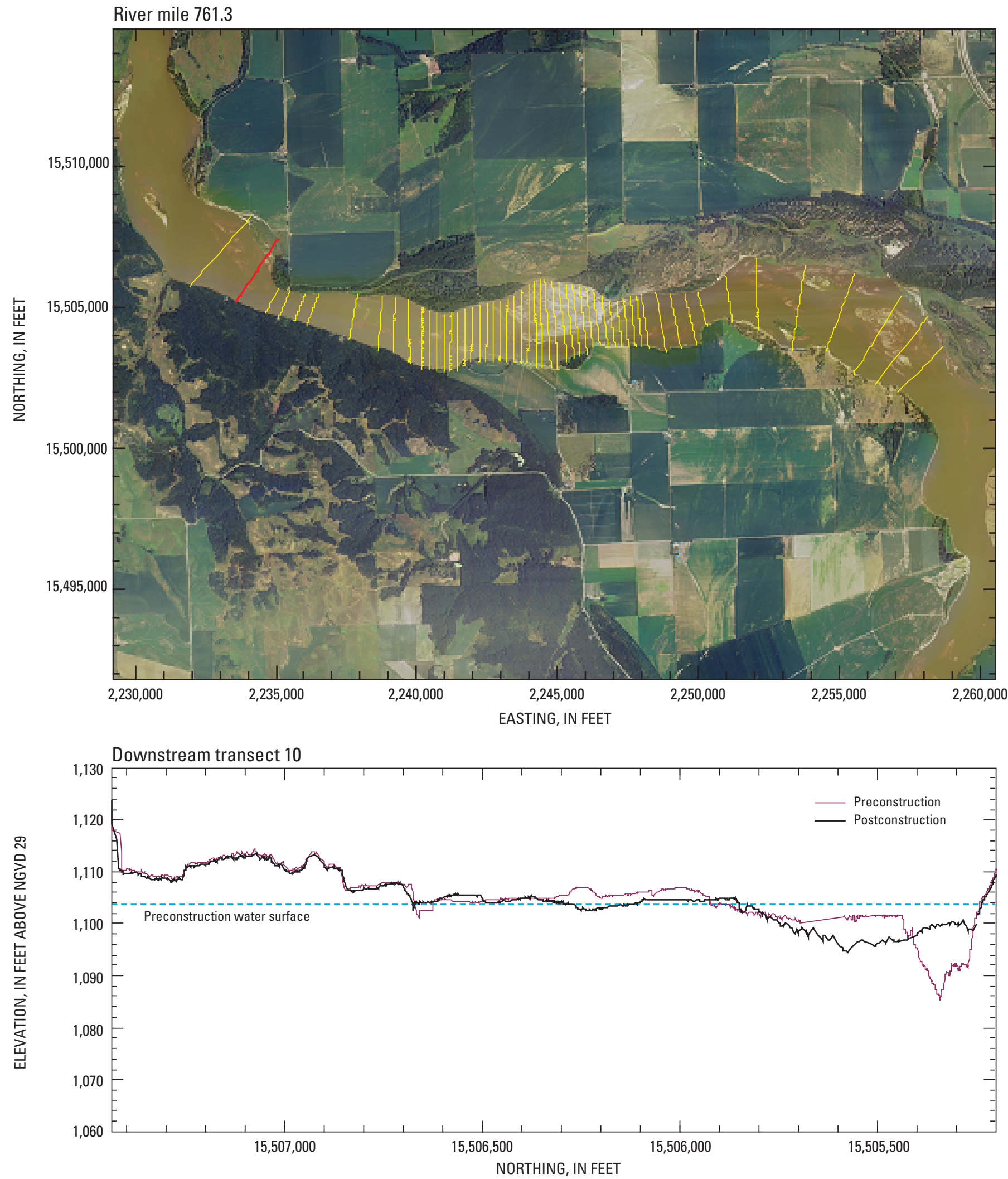

Base from Farm Service Agency digital orthophotography, 2003

Universal Transverse Mercator projection, Zone 14

Horizontal coordinates are referenced to the North American Datum of 1983 (NAD 83)

Vertical coordinates are referenced to the National Geodetic Vertical Datum of 1929 (NGVD 29)

Figure A2. Location and cross section for downstream transect 10. 

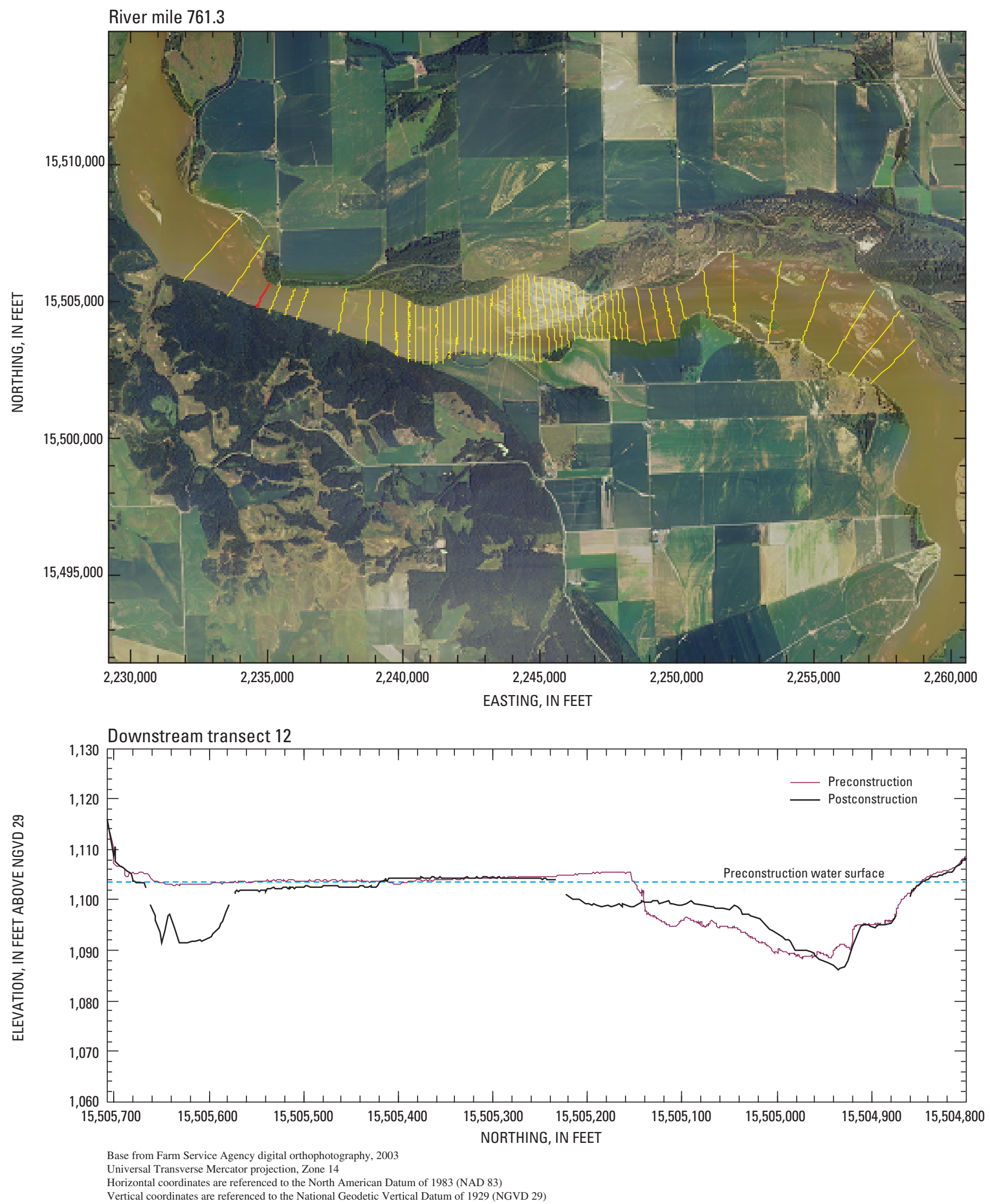

Figure A3. Location and cross section for downstream transect 12. 

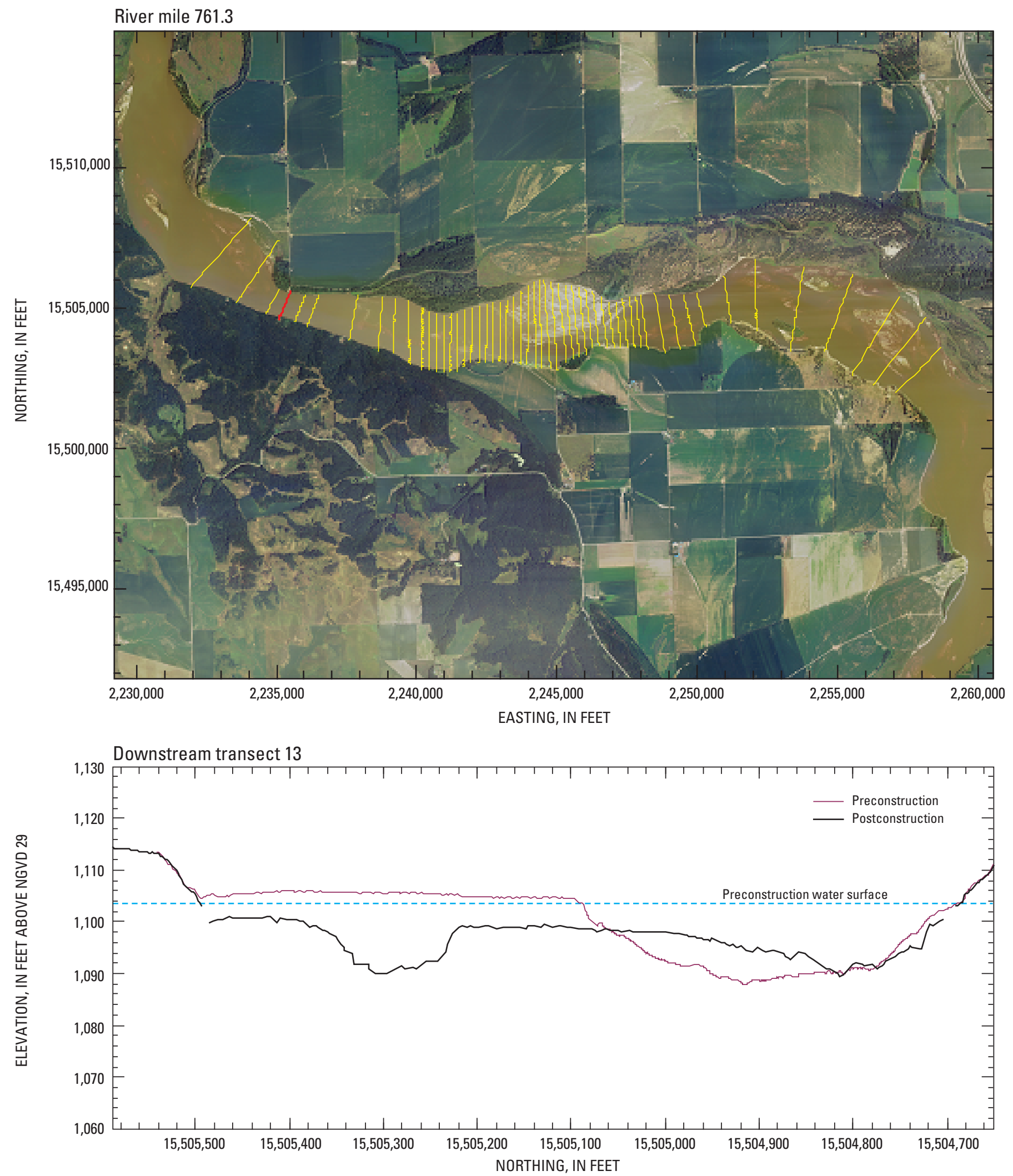

Base from Farm Service Agency digital orthophotography, 2003

Universal Transverse Mercator projection, Zone 14

Horizontal coordinates are referenced to the North American Datum of 1983 (NAD 83)

Vertical coordinates are referenced to the National Geodetic Vertical Datum of 1929 (NGVD 29)

Figure A4. Location and cross section for downstream transect 13. 

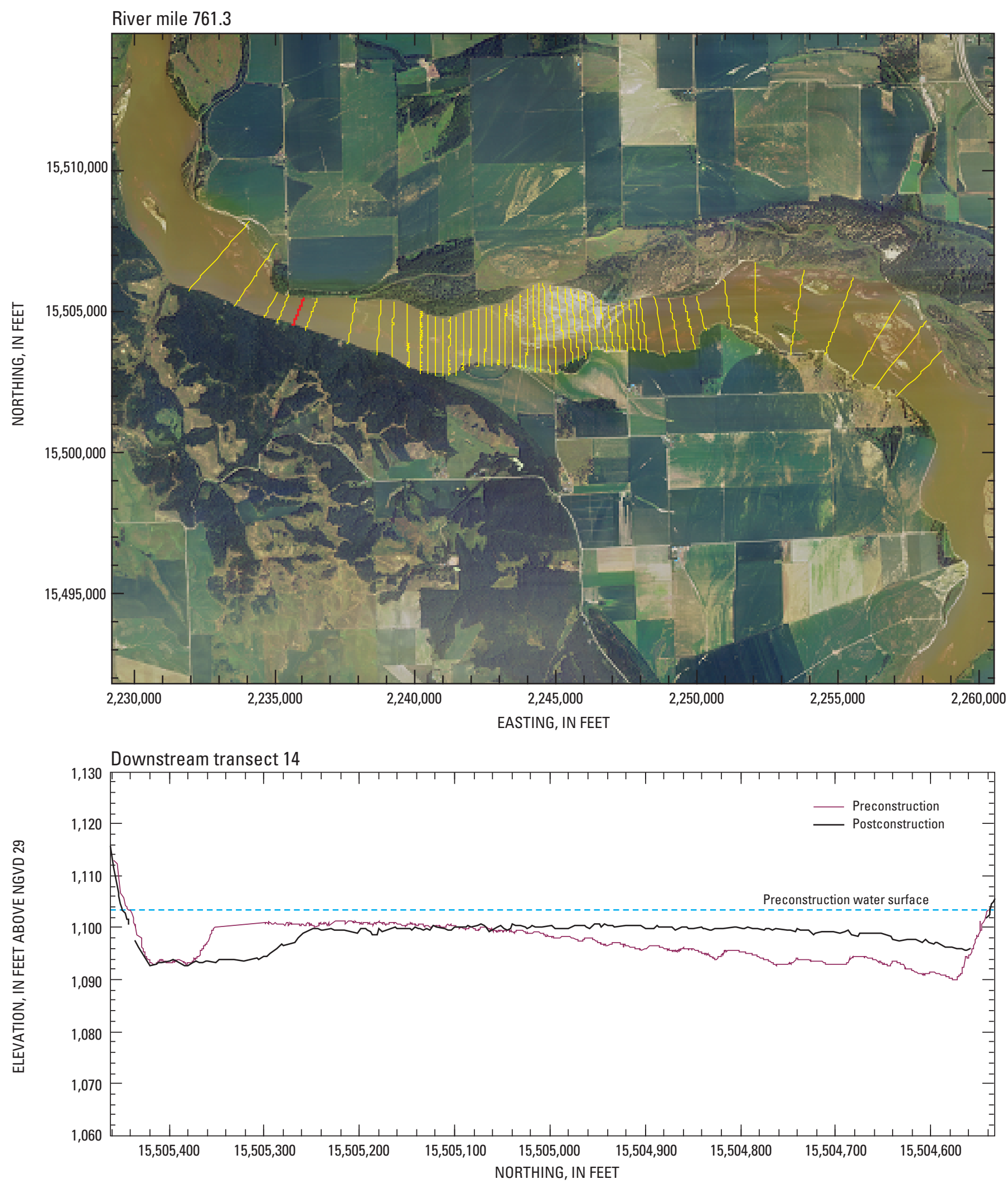

Base from Farm Service Agency digital orthophotography, 2003

Universal Transverse Mercator projection, Zone 14

Horizontal coordinates are referenced to the North American Datum of 1983 (NAD 83)

Vertical coordinates are referenced to the National Geodetic Vertical Datum of 1929 (NGVD 29)

Figure A5. Location and cross section for downstream transect 14. 

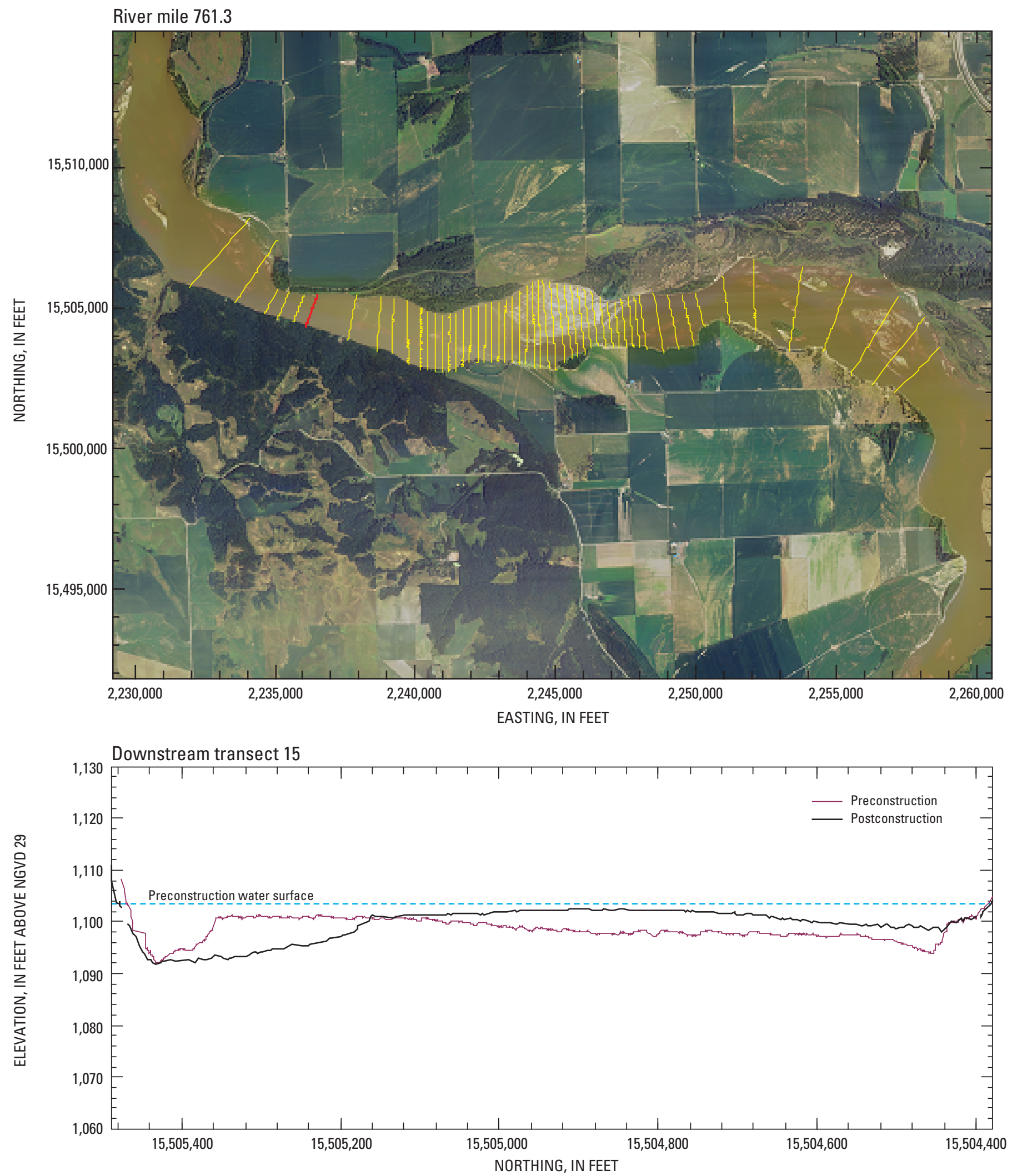

Base from Farm Service Agency digital orthophotography, 2003

Universal Transverse Mercator projection, Zone 14

Horizontal coordinates are referenced to the North American Datum of 1983 (NAD 83)

Vertical coordinates are referenced to the National Geodetic Vertical Datum of 1929 (NGVD 29)

Figure A6. Location and cross section for downstream transect 15. 

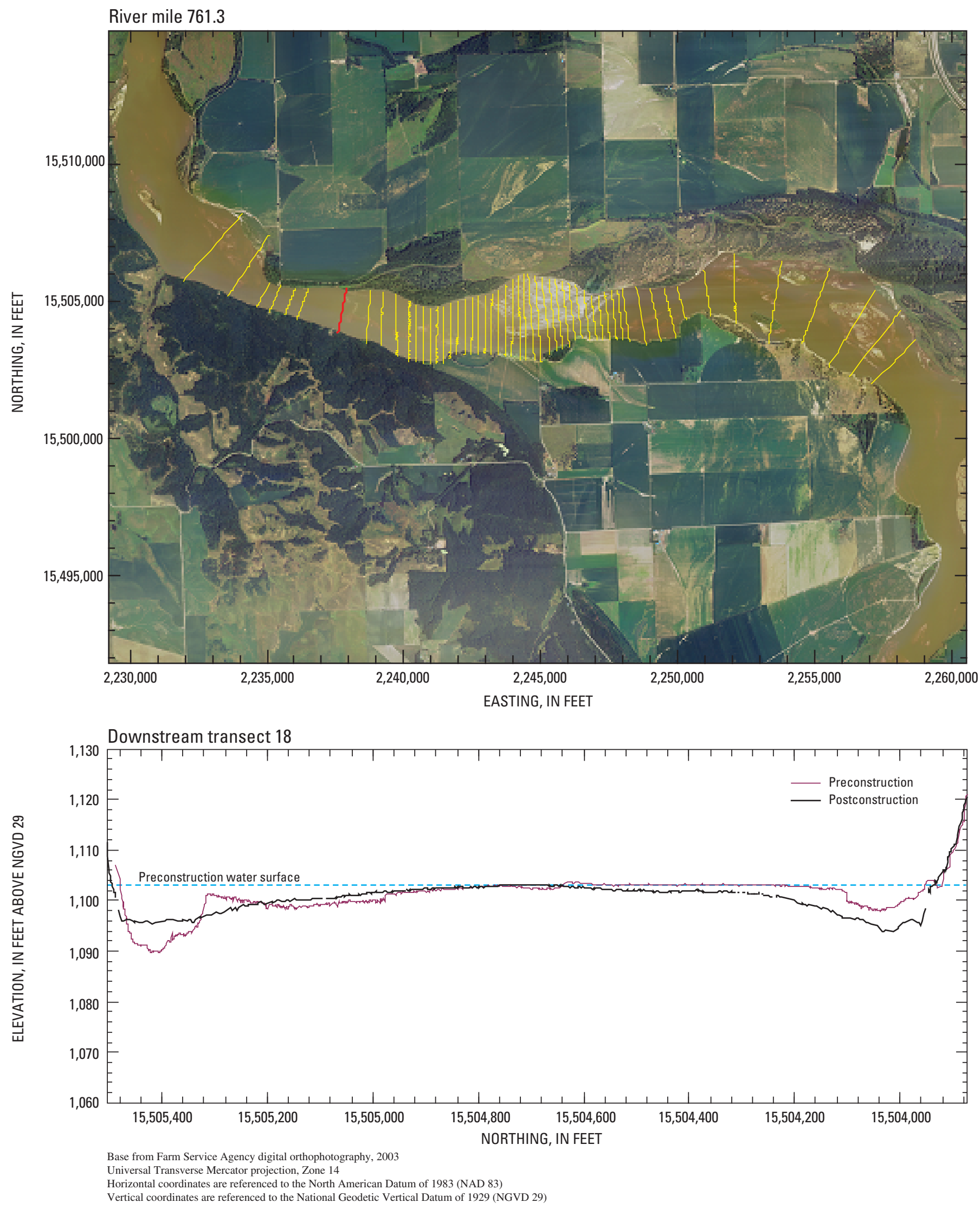

Figure A7. Location and cross section for downstream transect 18. 

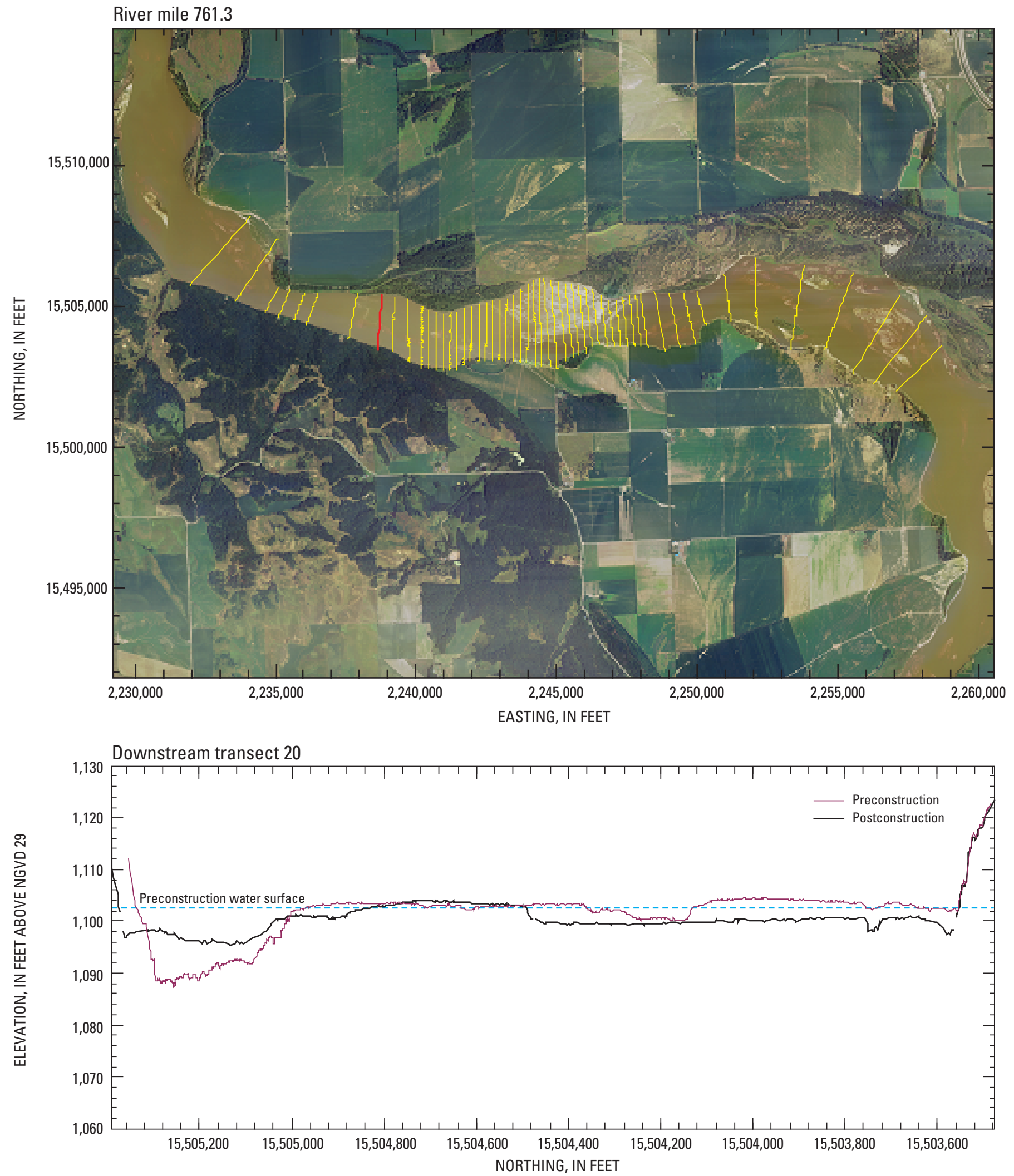

Base from Farm Service Agency digital orthophotography, 2003

Universal Transverse Mercator projection, Zone 14

Horizontal coordinates are referenced to the North American Datum of 1983 (NAD 83)

Vertical coordinates are referenced to the National Geodetic Vertical Datum of 1929 (NGVD 29)

Figure A8. Location and cross section for downstream transect 20. 

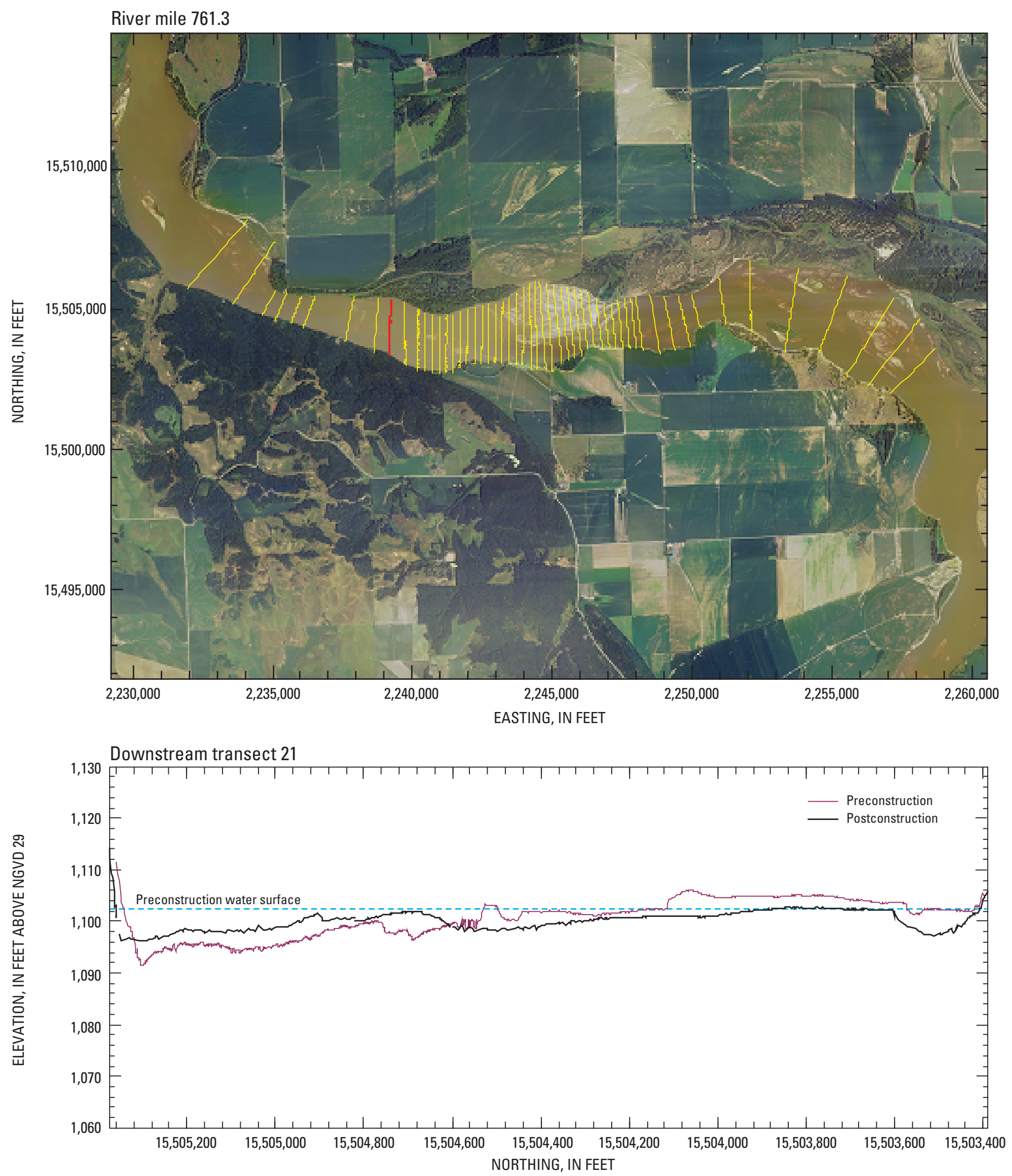

Base from Farm Service Agency digital orthophotography, 2003

Universal Transverse Mercator projection, Zone 14

Horizontal coordinates are referenced to the North American Datum of 1983 (NAD 83)

Vertical coordinates are referenced to the National Geodetic Vertical Datum of 1929 (NGVD 29)

Figure A9. Location and cross section for downstream transect 21. 

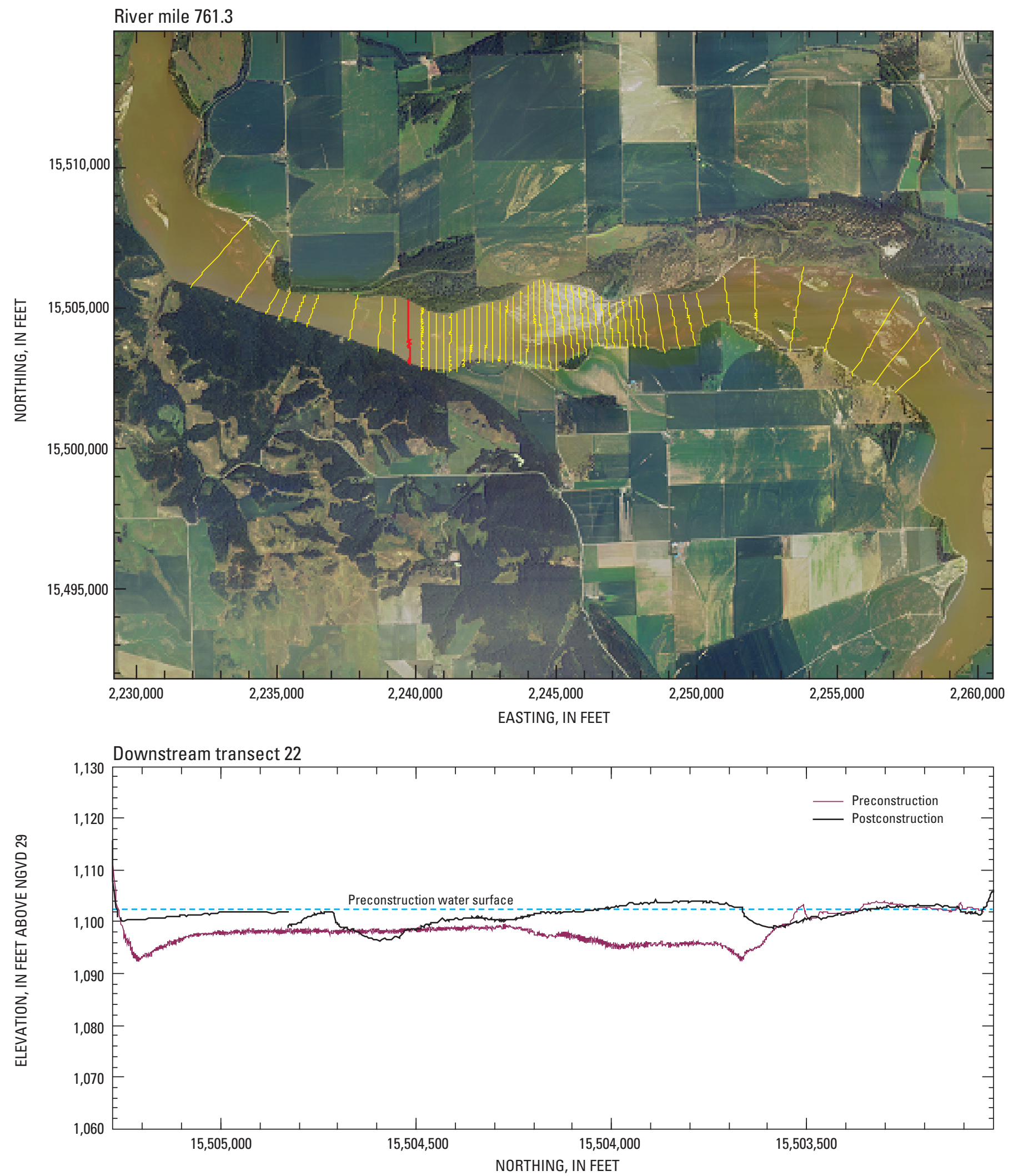

Base from Farm Service Agency digital orthophotography, 2003

Universal Transverse Mercator projection, Zone 14

Horizontal coordinates are referenced to the North American Datum of 1983 (NAD 83)

Vertical coordinates are referenced to the National Geodetic Vertical Datum of 1929 (NGVD 29)

Figure A10. Location and cross section for downstream transect 22. 

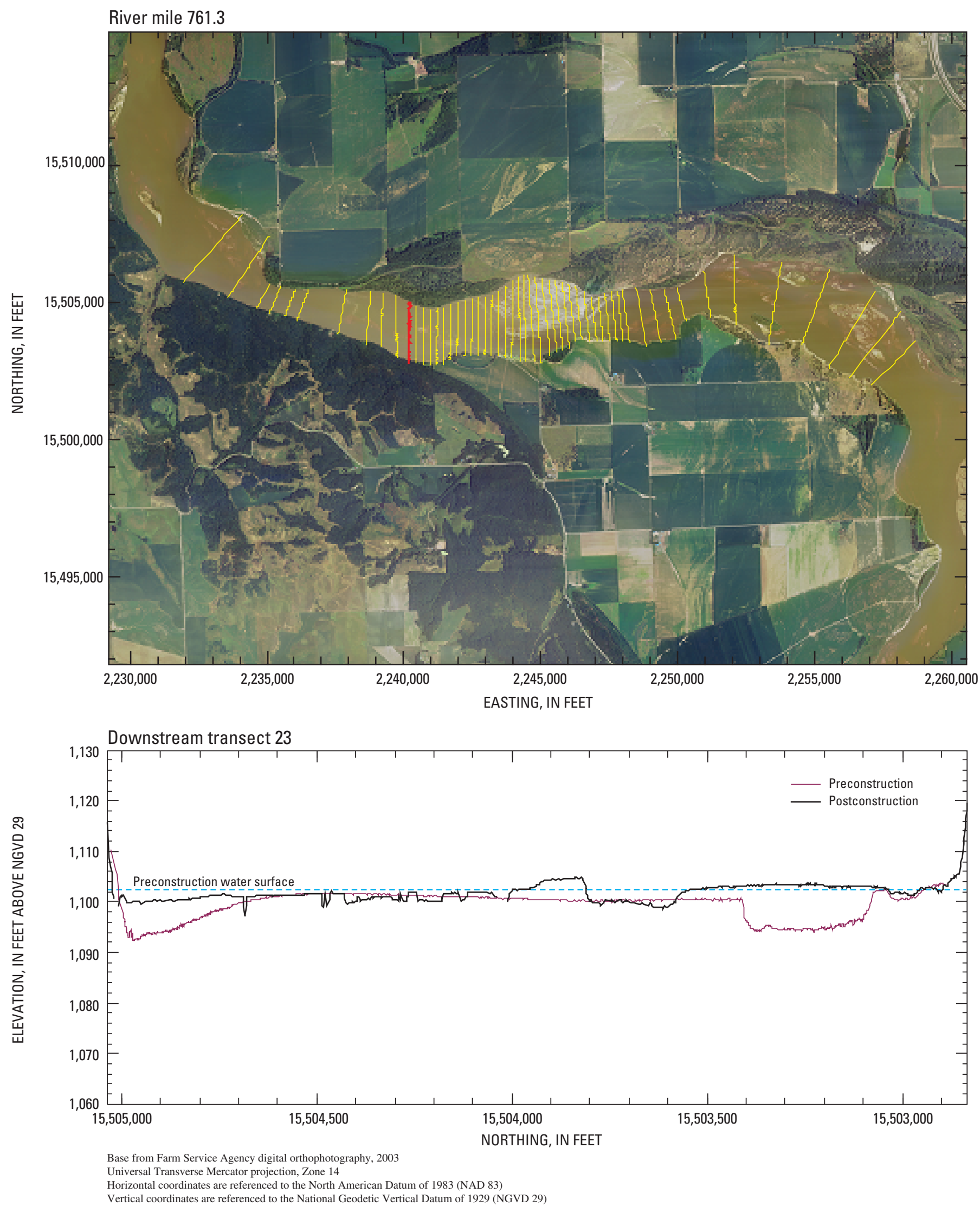

Figure A11. Location and cross section for downstream transect 23. 

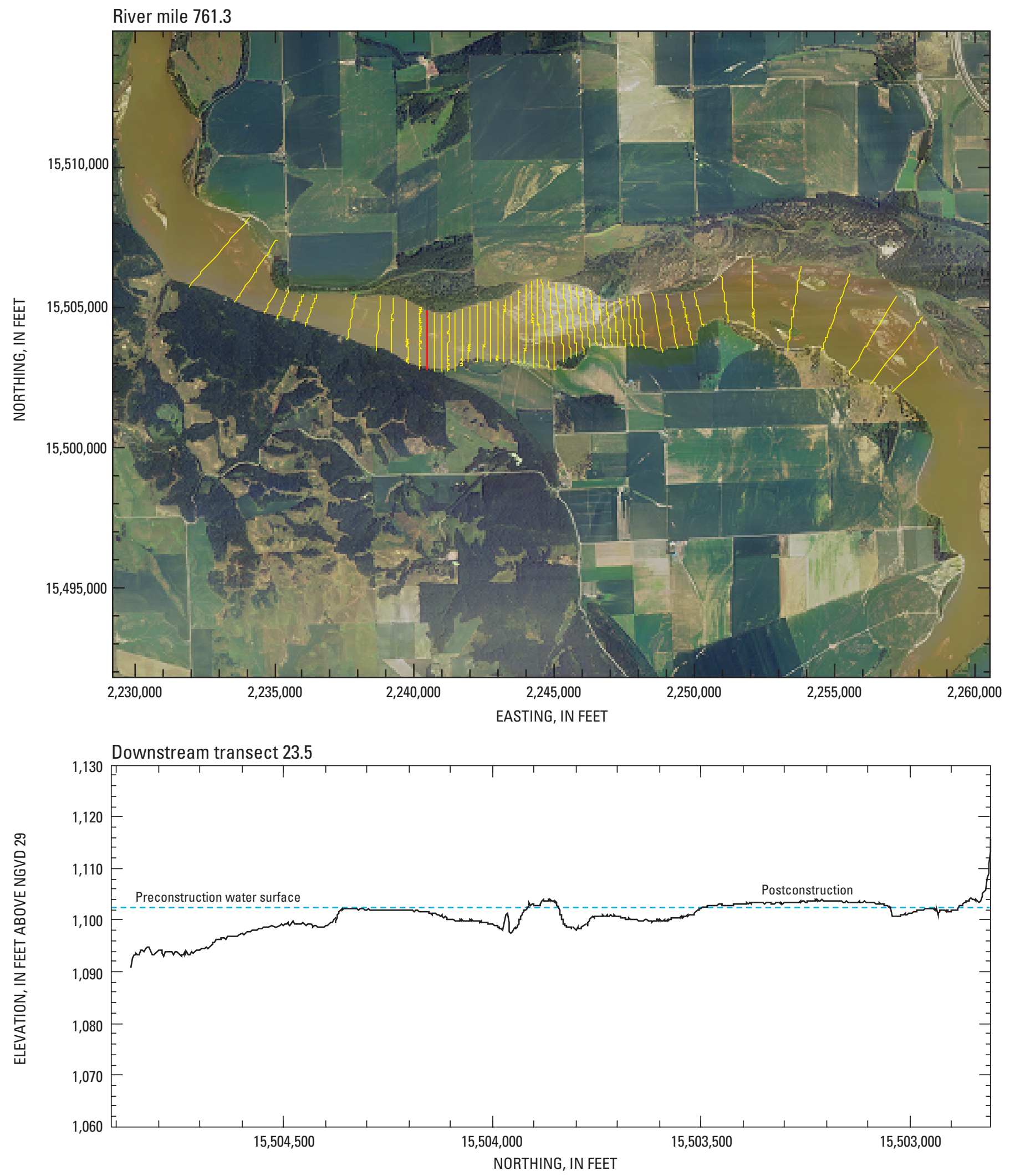

Base from Farm Service Agency digital orthophotography, 2003

Universal Transverse Mercator projection, Zone 14

Horizontal coordinates are referenced to the North American Datum of 1983 (NAD 83)

Vertical coordinates are referenced to the National Geodetic Vertical Datum of 1929 (NGVD 29)

Figure A12. Location and cross section for downstream transect 23.5. 

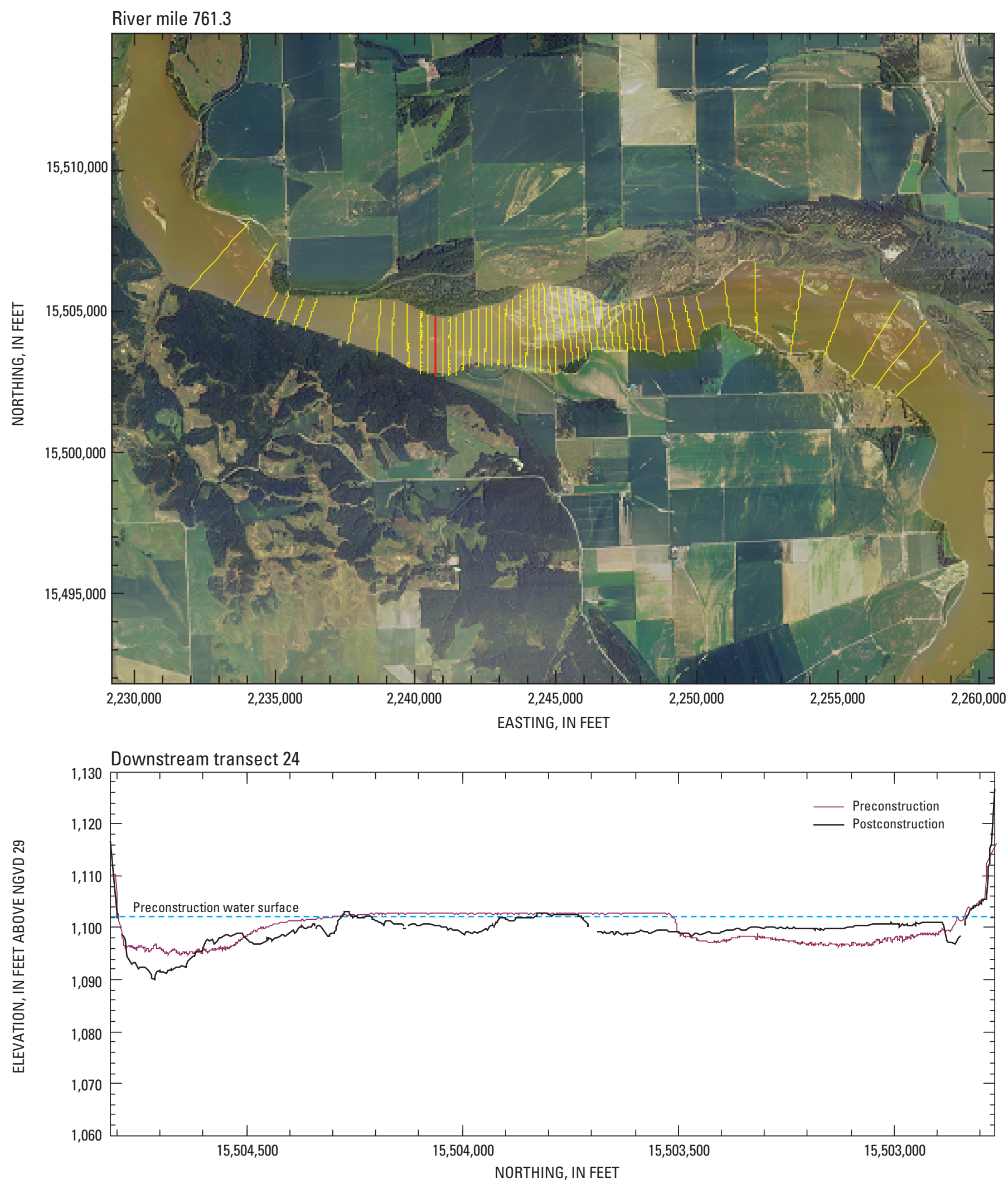

Base from Farm Service Agency digital orthophotography, 2003

Universal Transverse Mercator projection, Zone 14

Horizontal coordinates are referenced to the North American Datum of 1983 (NAD 83)

Vertical coordinates are referenced to the National Geodetic Vertical Datum of 1929 (NGVD 29)

Figure A13. Location and cross section for downstream transect 24. 

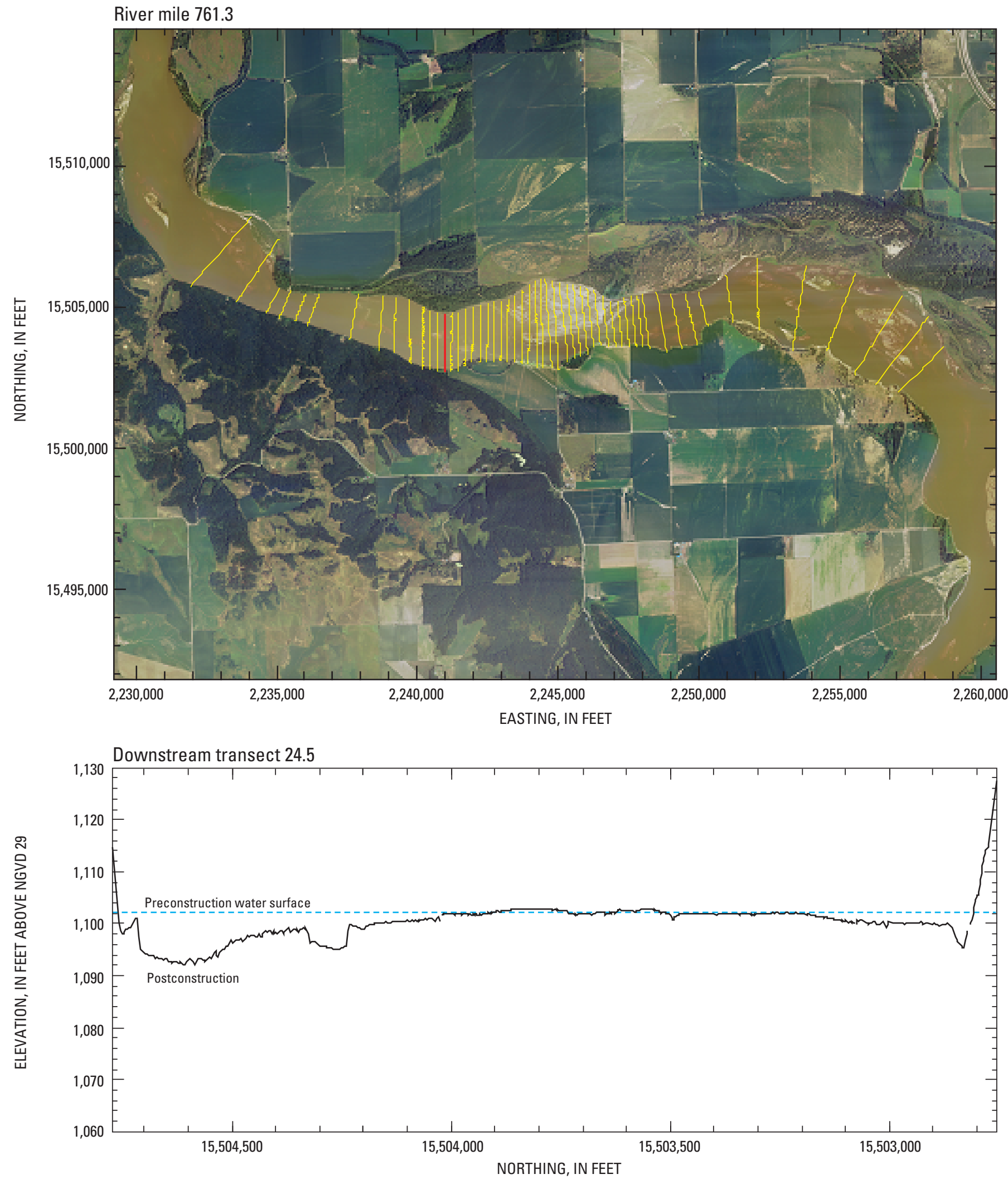

Base from Farm Service Agency digital orthophotography, 2003

Universal Transverse Mercator projection, Zone 14

Horizontal coordinates are referenced to the North American Datum of 1983 (NAD 83)

Vertical coordinates are referenced to the National Geodetic Vertical Datum of 1929 (NGVD 29)

Figure A14. Location and cross section for downstream transect 24.5. 

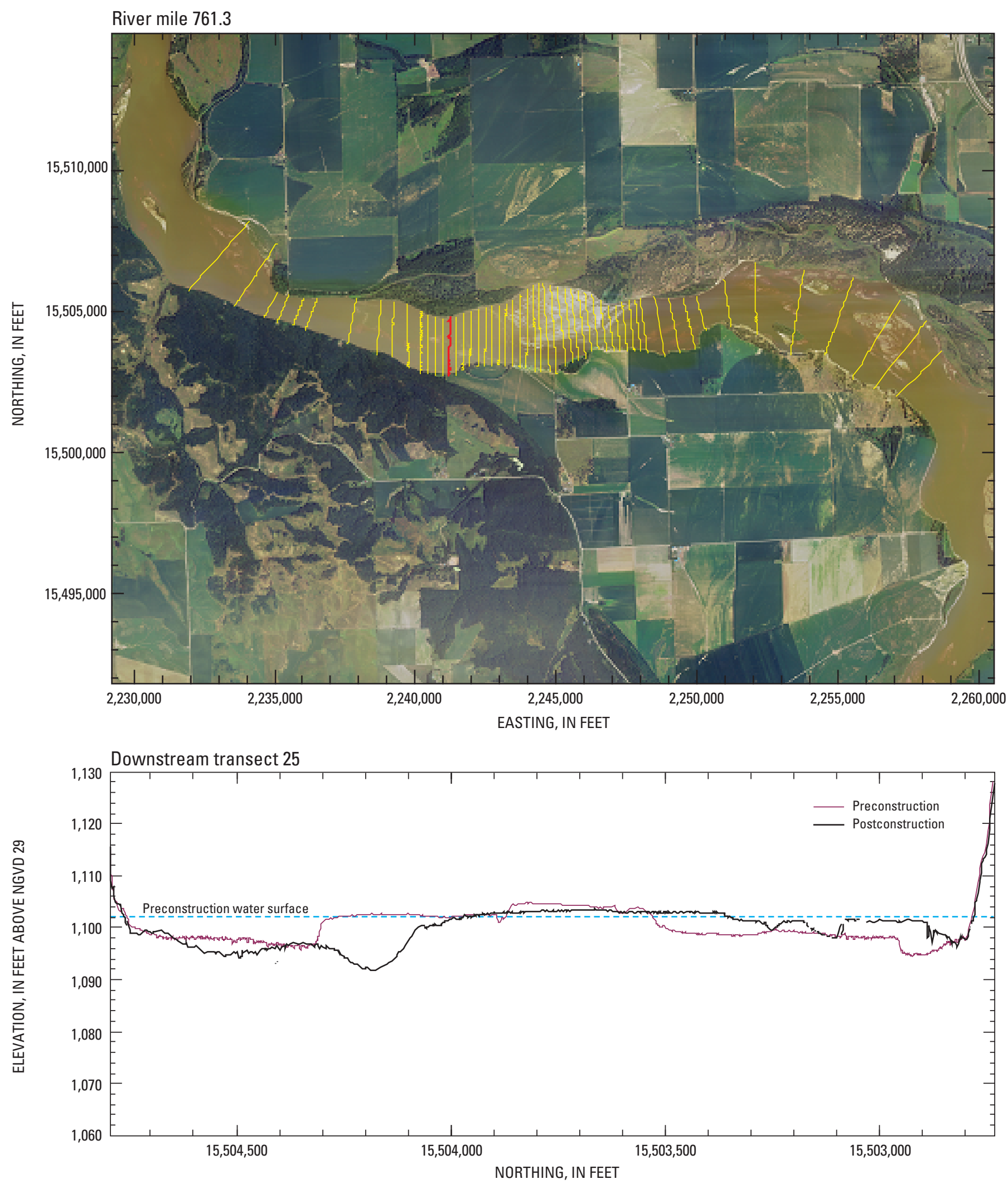

Base from Farm Service Agency digital orthophotography, 2003

Universal Transverse Mercator projection, Zone 14

Horizontal coordinates are referenced to the North American Datum of 1983 (NAD 83)

Vertical coordinates are referenced to the National Geodetic Vertical Datum of 1929 (NGVD 29)

Figure A15. Location and cross section for downstream transect 25. 

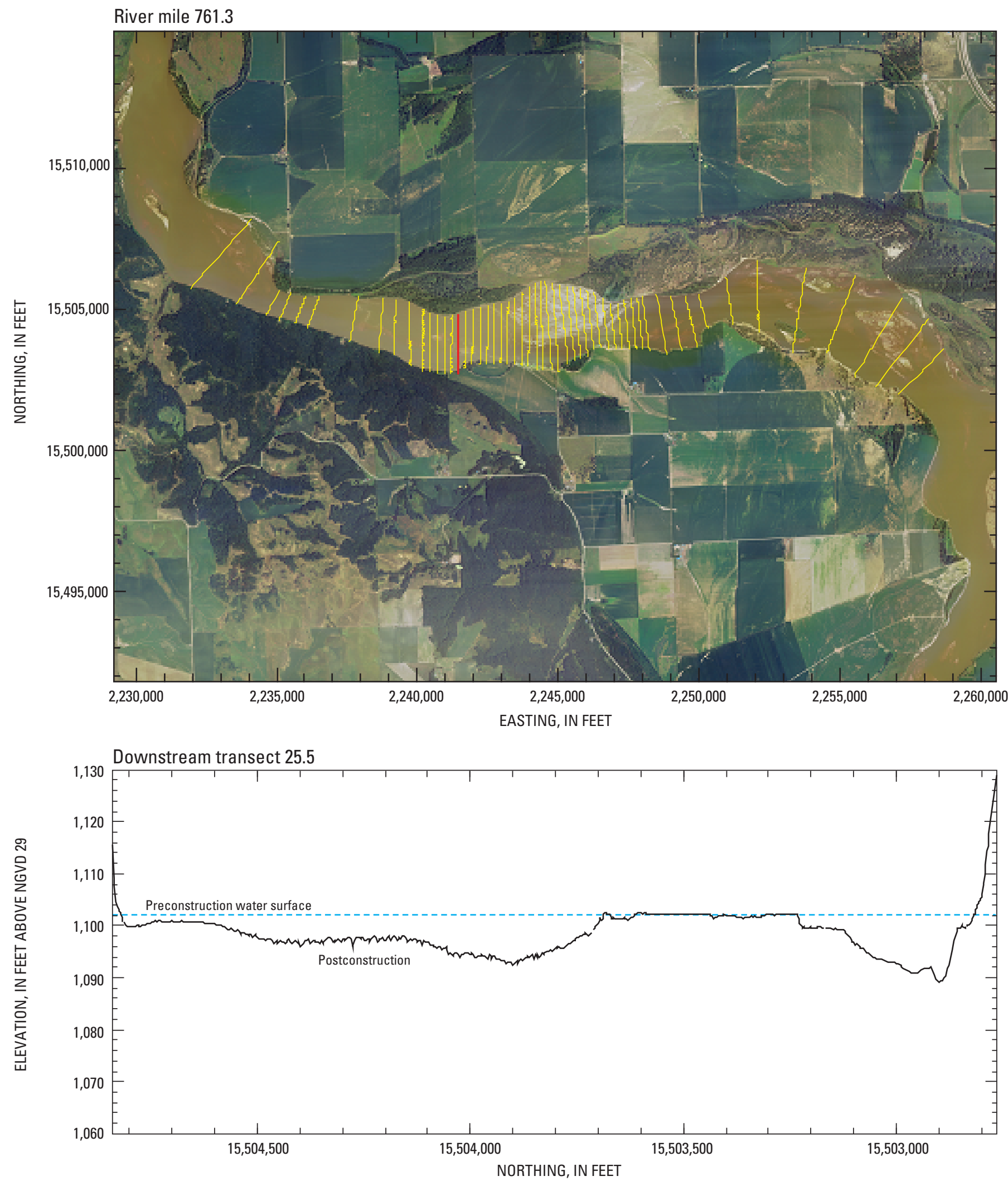

Base from Farm Service Agency digital orthophotography, 2003

Universal Transverse Mercator projection, Zone 14

Horizontal coordinates are referenced to the North American Datum of 1983 (NAD 83)

Vertical coordinates are referenced to the National Geodetic Vertical Datum of 1929 (NGVD 29)

Figure A16. Location and cross section for downstream transect 25.5. 

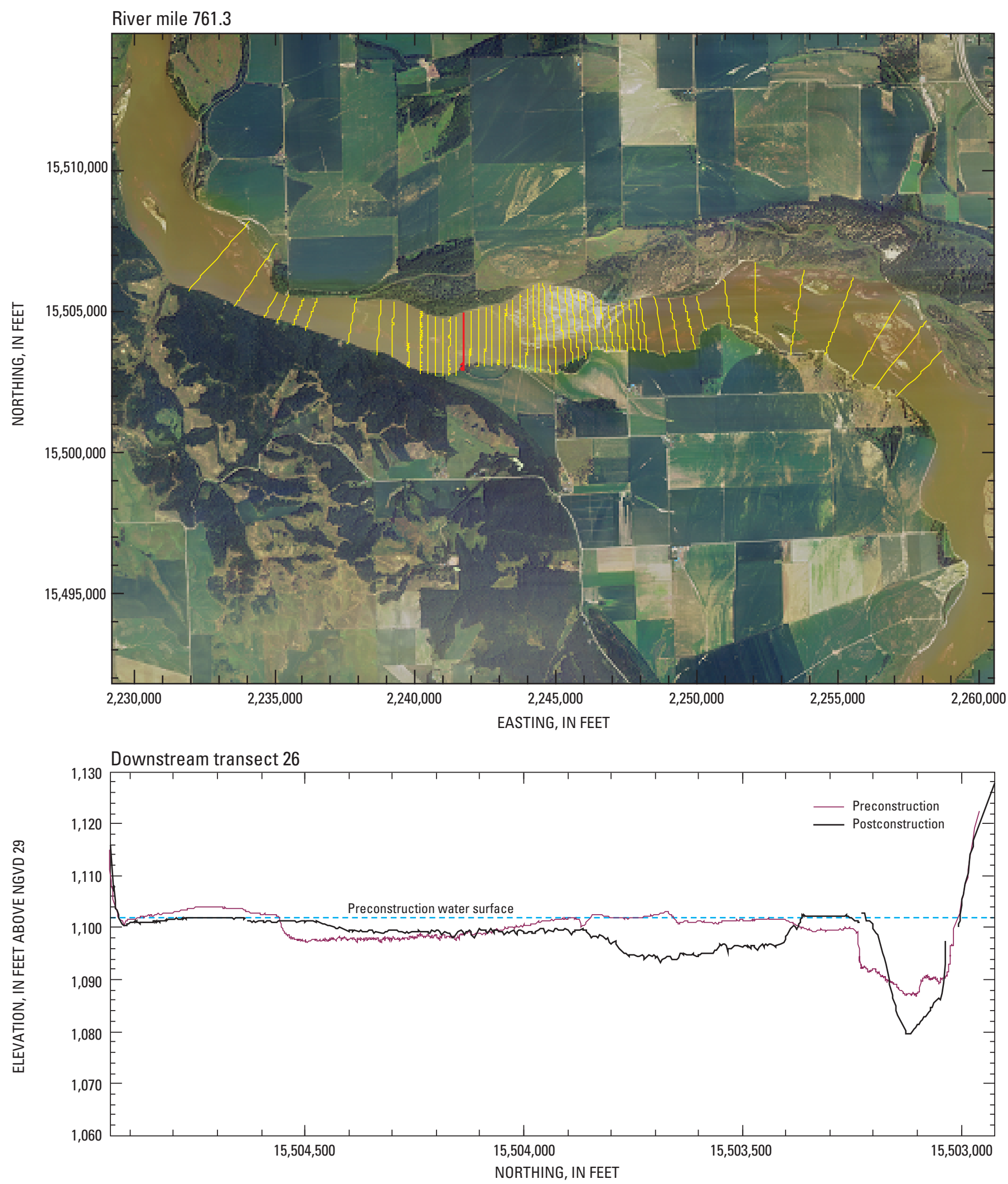

Base from Farm Service Agency digital orthophotography, 2003

Universal Transverse Mercator projection, Zone 14

Horizontal coordinates are referenced to the North American Datum of 1983 (NAD 83)

Vertical coordinates are referenced to the National Geodetic Vertical Datum of 1929 (NGVD 29)

Figure A17. Location and cross section for downstream transect 26. 

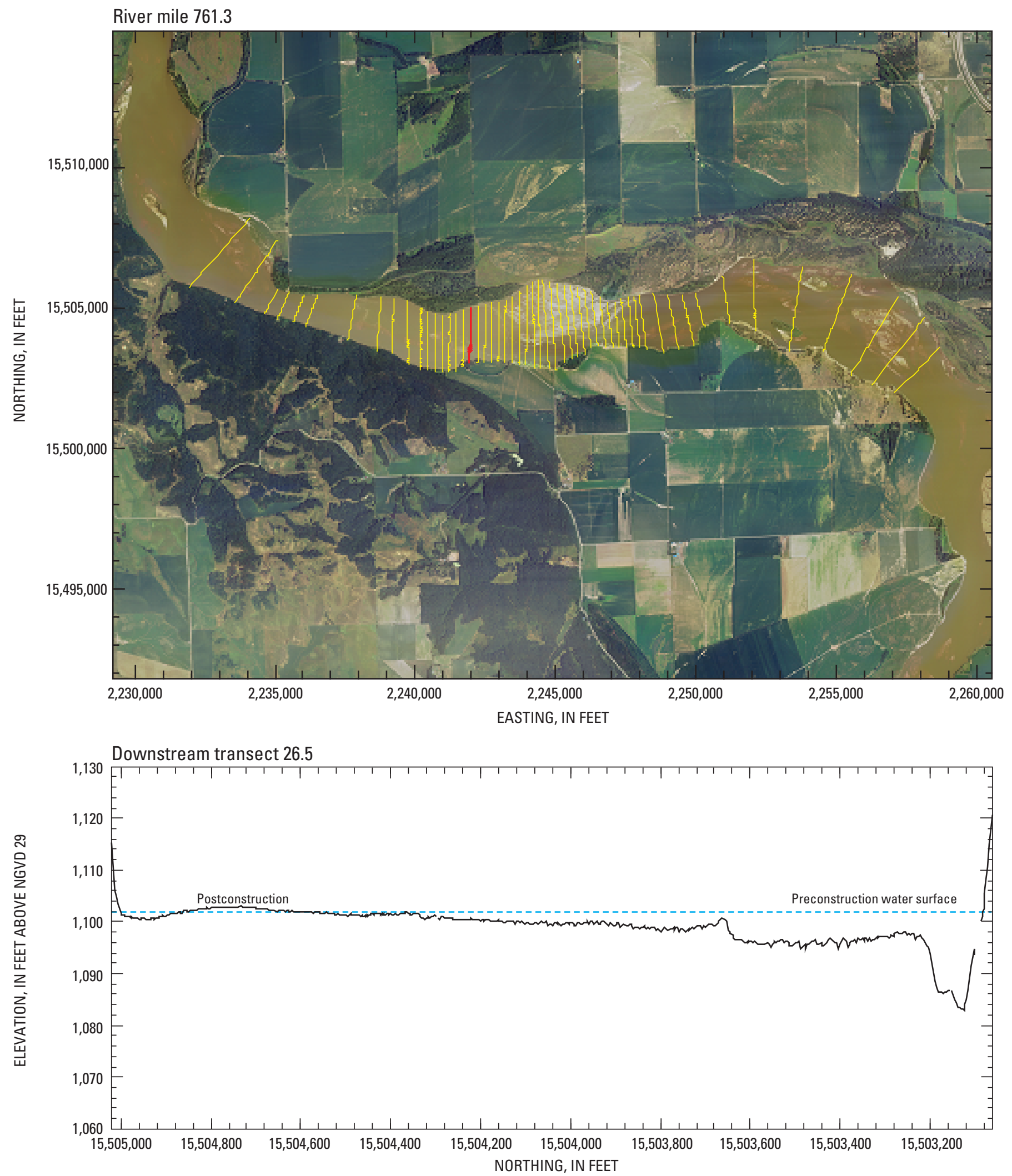

Base from Farm Service Agency digital orthophotography, 2003

Universal Transverse Mercator projection, Zone 14

Horizontal coordinates are referenced to the North American Datum of 1983 (NAD 83)

Vertical coordinates are referenced to the National Geodetic Vertical Datum of 1929 (NGVD 29)

Figure A18. Location and cross section for downstream transect 26.5. 

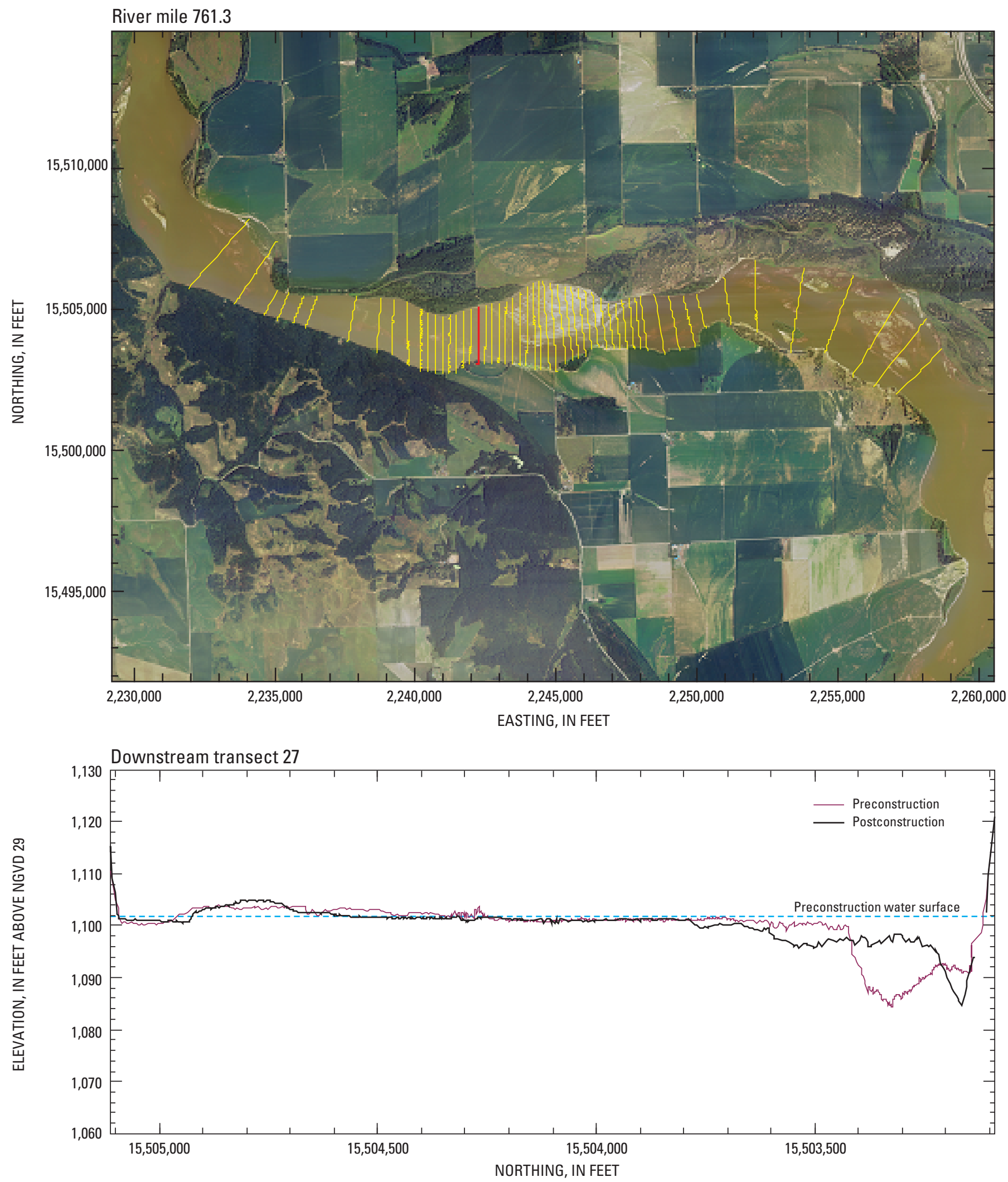

Base from Farm Service Agency digital orthophotography, 2003

Universal Transverse Mercator projection, Zone 14

Horizontal coordinates are referenced to the North American Datum of 1983 (NAD 83)

Vertical coordinates are referenced to the National Geodetic Vertical Datum of 1929 (NGVD 29)

Figure A19. Location and cross section for downstream transect 27. 

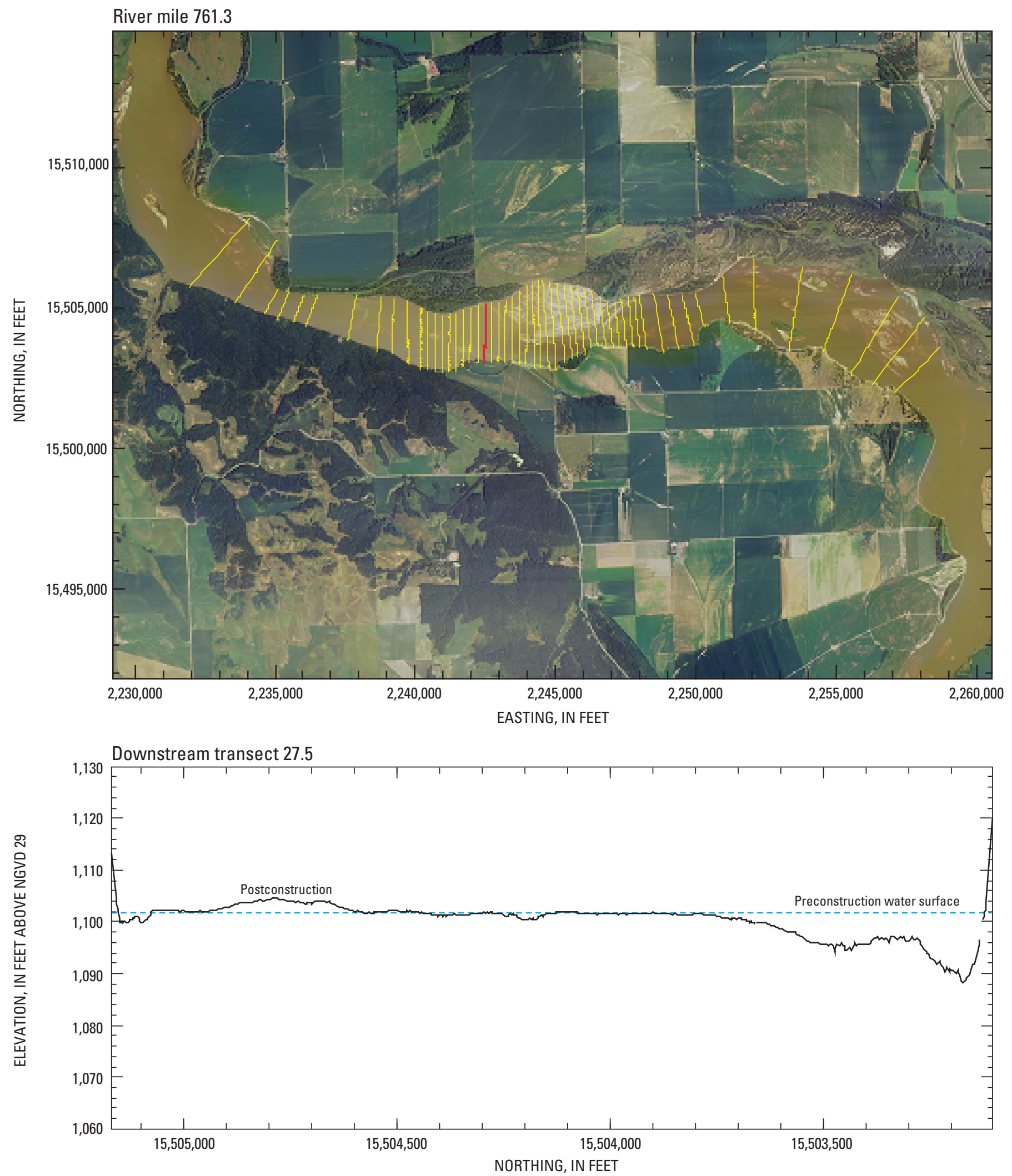

Base from Farm Service Agency digital orthophotography, 2003

Universal Transverse Mercator projection, Zone 14

Horizontal coordinates are referenced to the North American Datum of 1983 (NAD 83)

Vertical coordinates are referenced to the National Geodetic Vertical Datum of 1929 (NGVD 29)

Figure A20. Location and cross section for downstream transect 27.5. 

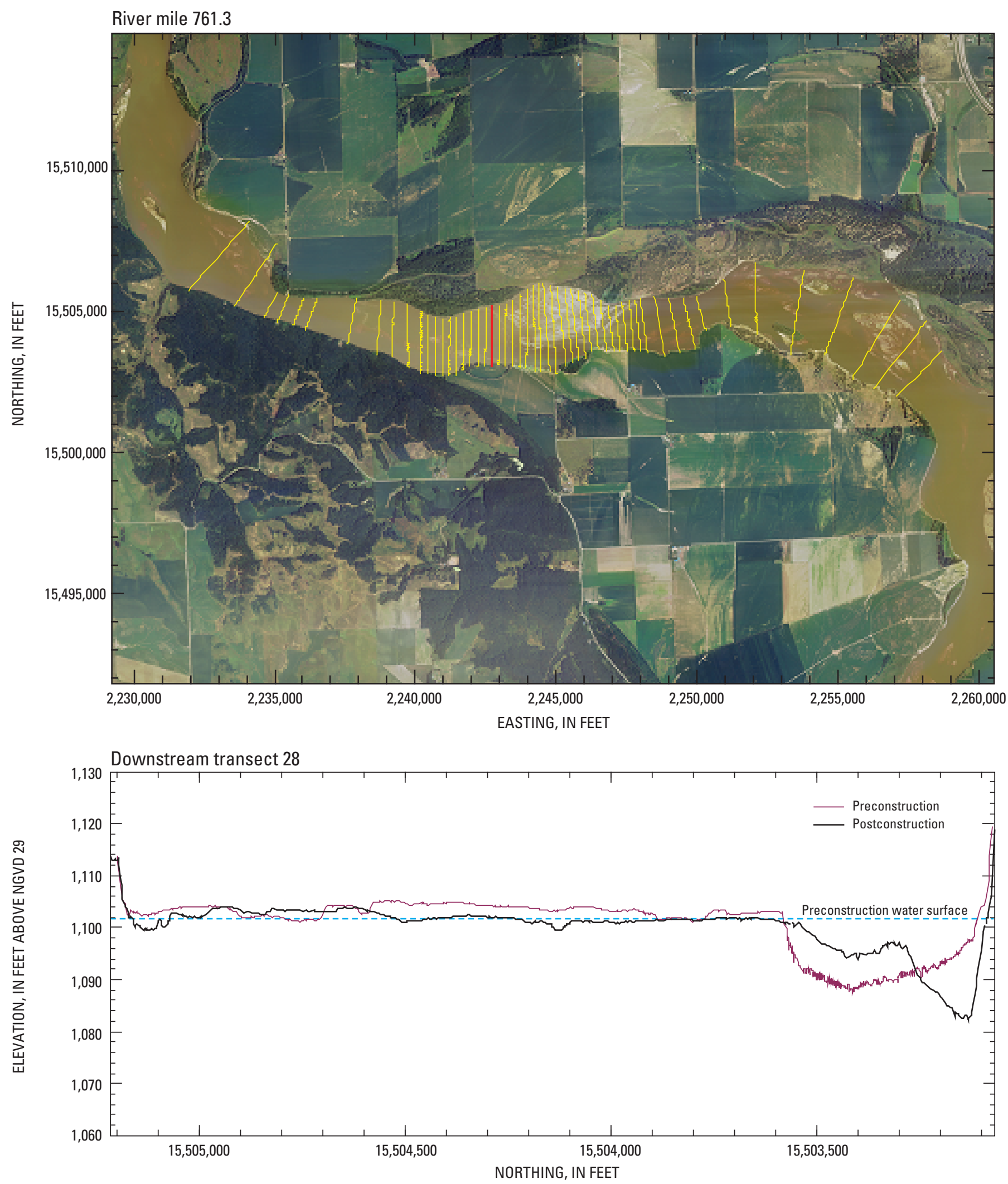

Base from Farm Service Agency digital orthophotography, 2003

Universal Transverse Mercator projection, Zone 14

Horizontal coordinates are referenced to the North American Datum of 1983 (NAD 83)

Vertical coordinates are referenced to the National Geodetic Vertical Datum of 1929 (NGVD 29)

Figure A21. Location and cross section for downstream transect 28. 

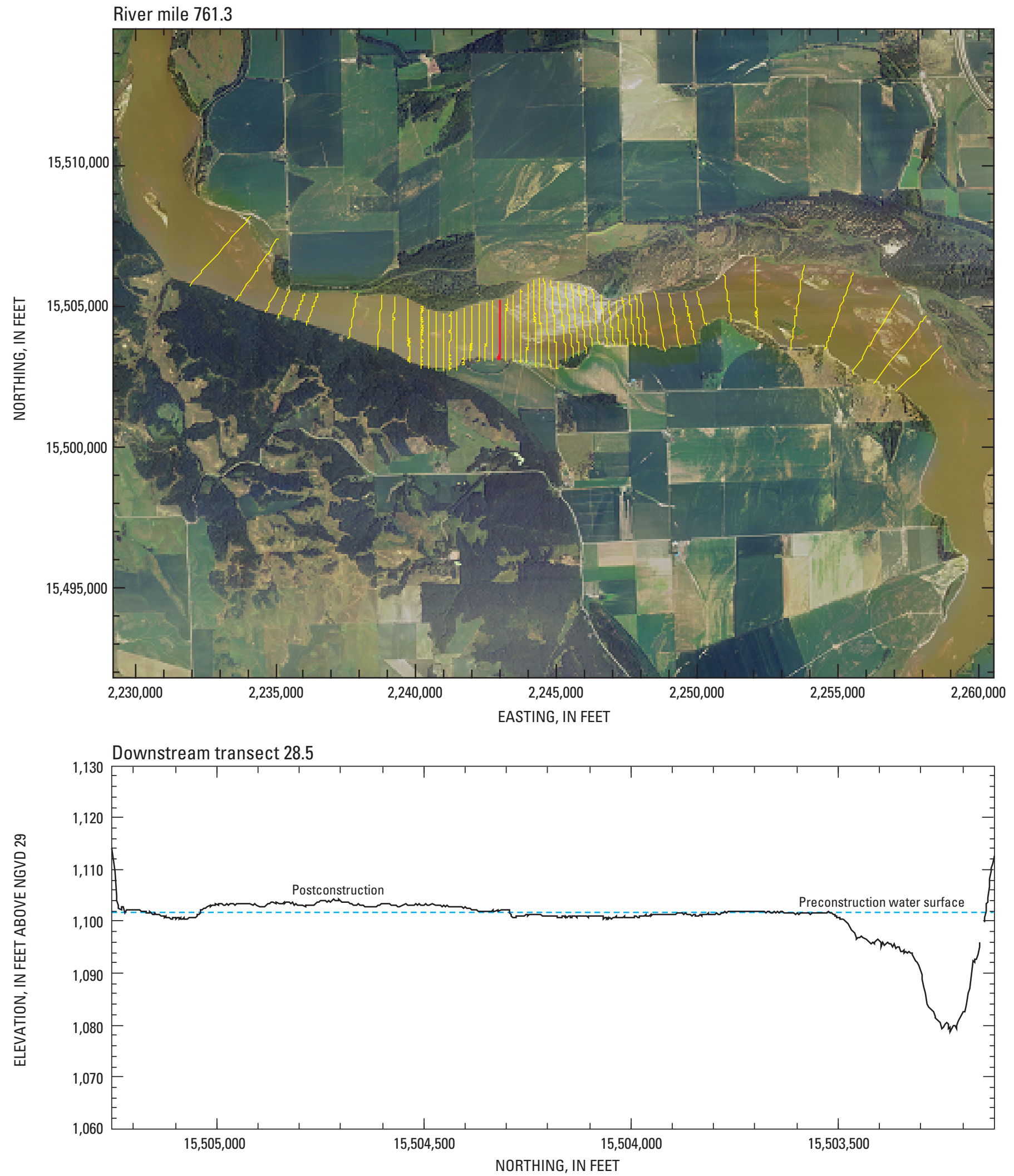

Base from Farm Service Agency digital orthophotography, 2003

Universal Transverse Mercator projection, Zone 14

Horizontal coordinates are referenced to the North American Datum of 1983 (NAD 83)

Vertical coordinates are referenced to the National Geodetic Vertical Datum of 1929 (NGVD 29)

Figure A22. Location and cross section for downstream transect 28.5. 

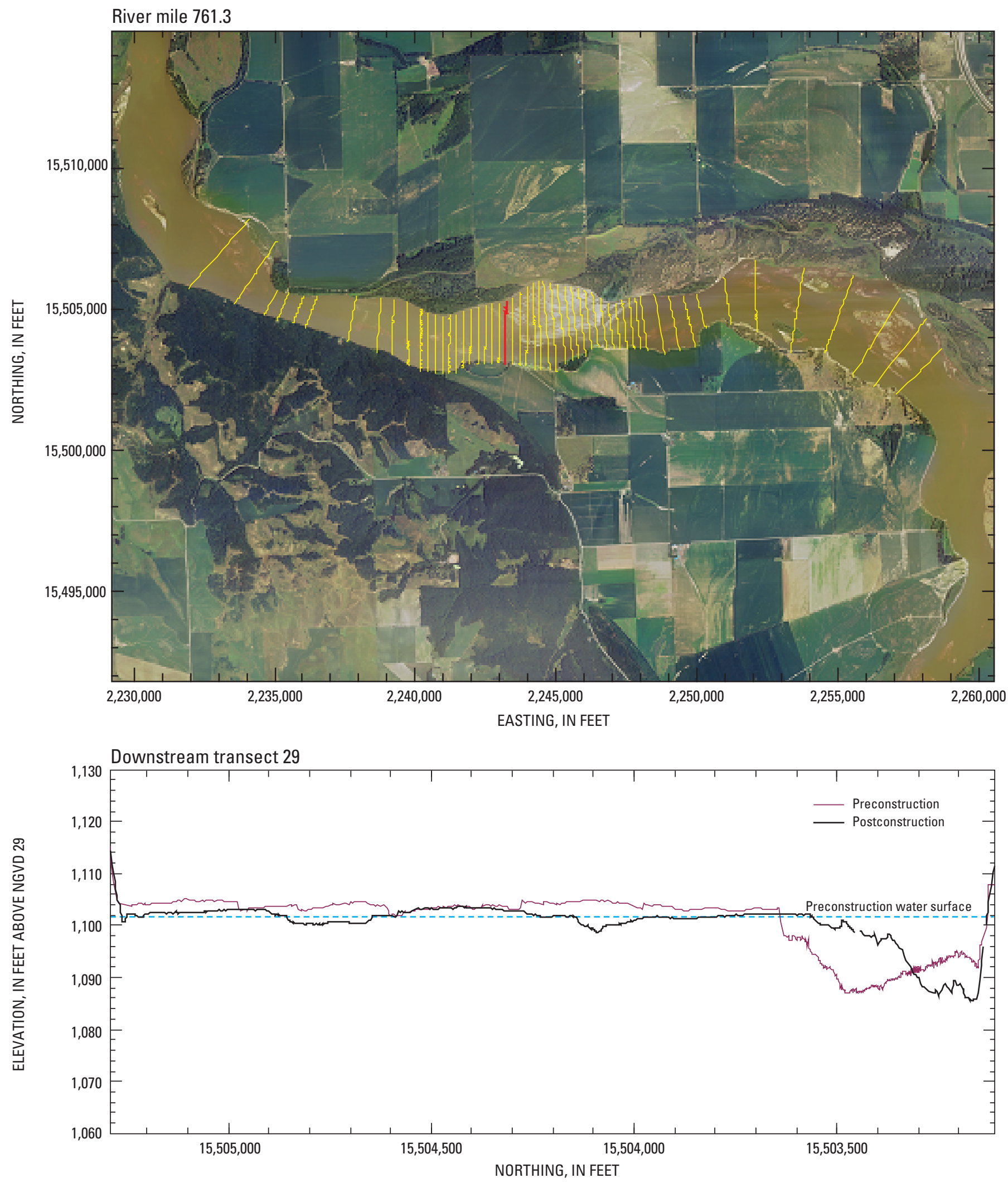

Base from Farm Service Agency digital orthophotography, 2003

Universal Transverse Mercator projection, Zone 14

Horizontal coordinates are referenced to the North American Datum of 1983 (NAD 83)

Vertical coordinates are referenced to the National Geodetic Vertical Datum of 1929 (NGVD 29)

Figure A23. Location and cross section for downstream transect 29. 

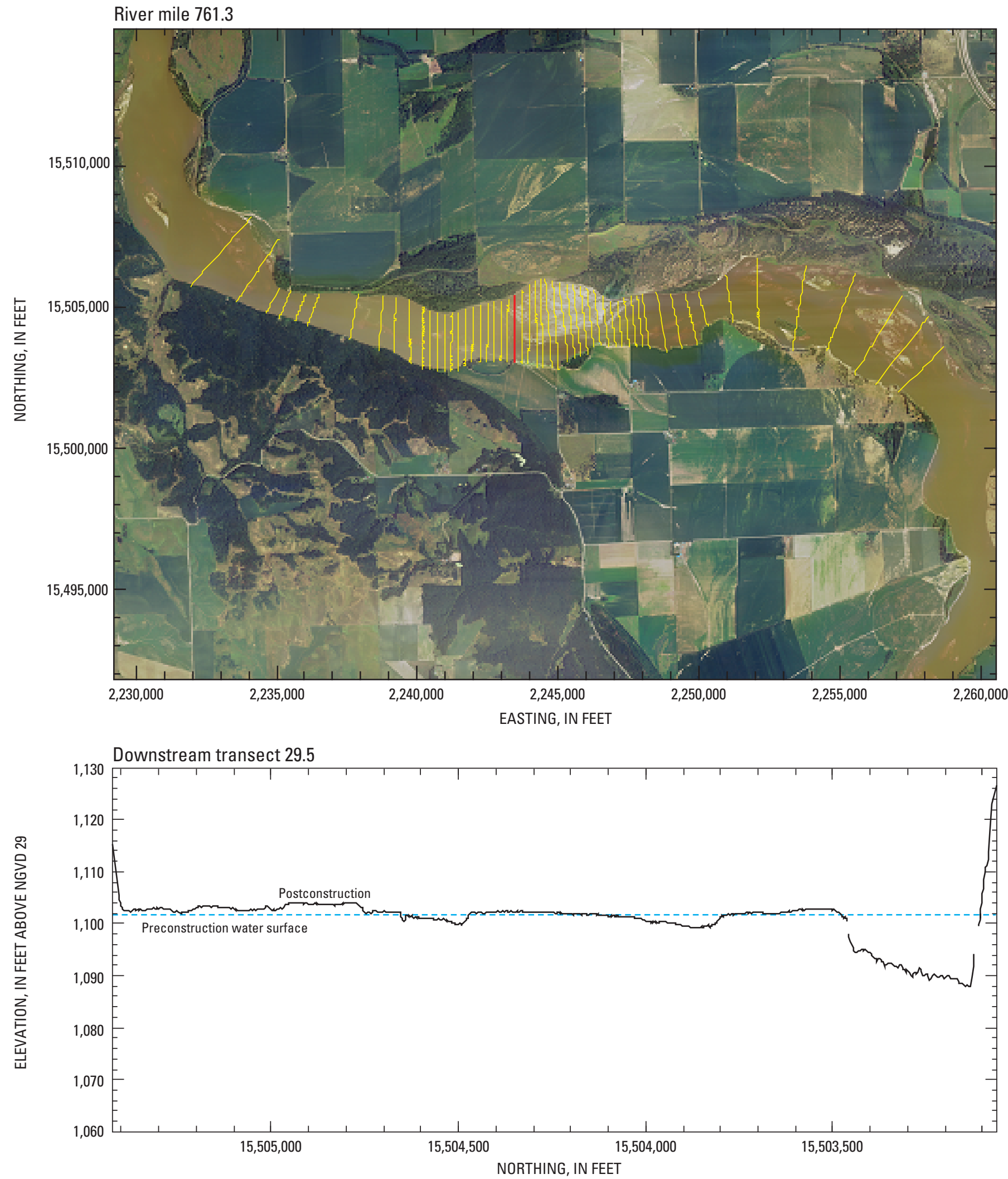

Base from Farm Service Agency digital orthophotography, 2003

Universal Transverse Mercator projection, Zone 14

Horizontal coordinates are referenced to the North American Datum of 1983 (NAD 83)

Vertical coordinates are referenced to the National Geodetic Vertical Datum of 1929 (NGVD 29)

Figure A24. Location and cross section for downstream transect 29.5. 



Figure A25. Location and cross section for downstream transect 30. 

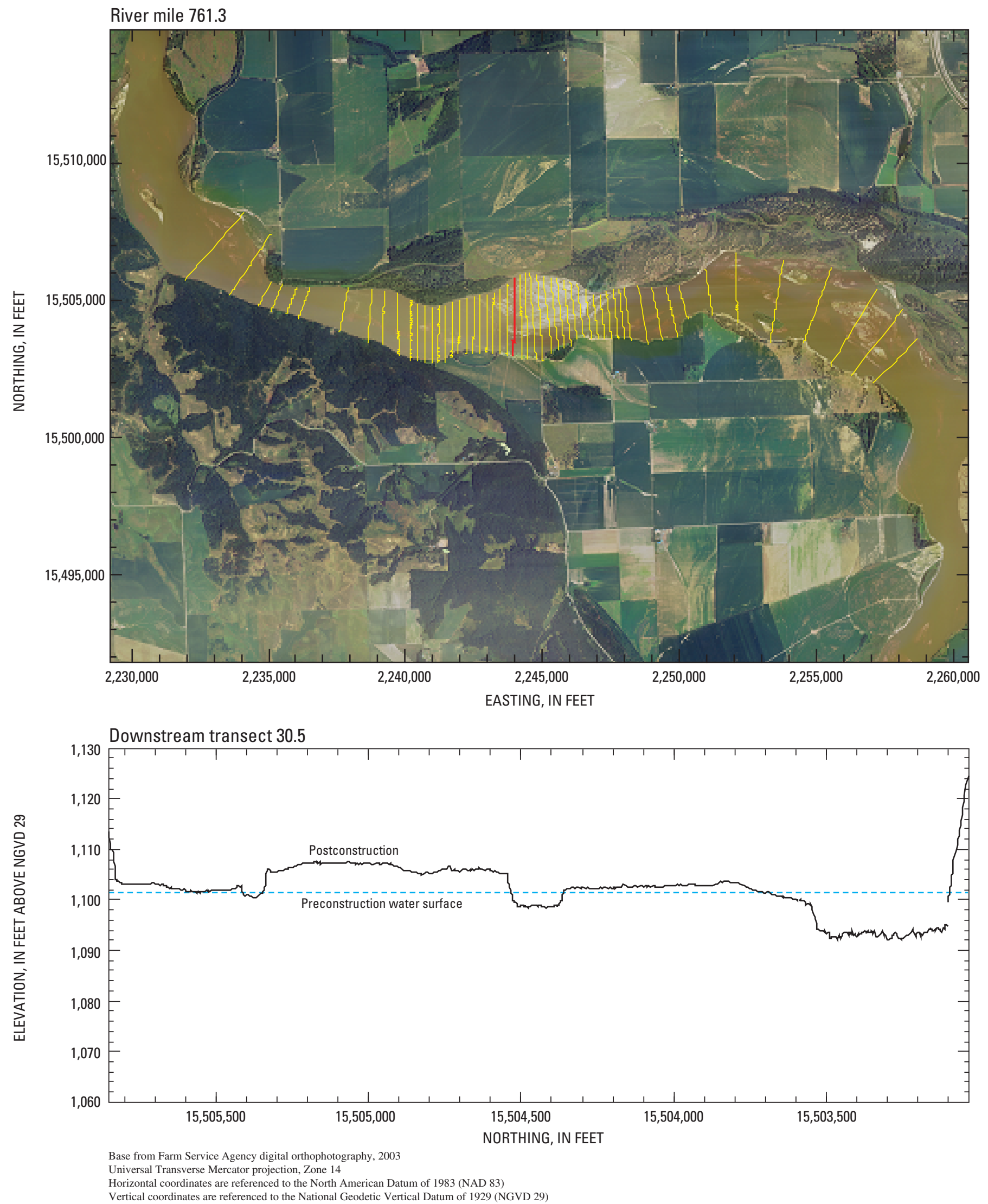

Figure A26. Location and cross section for downstream transect 30.5. 

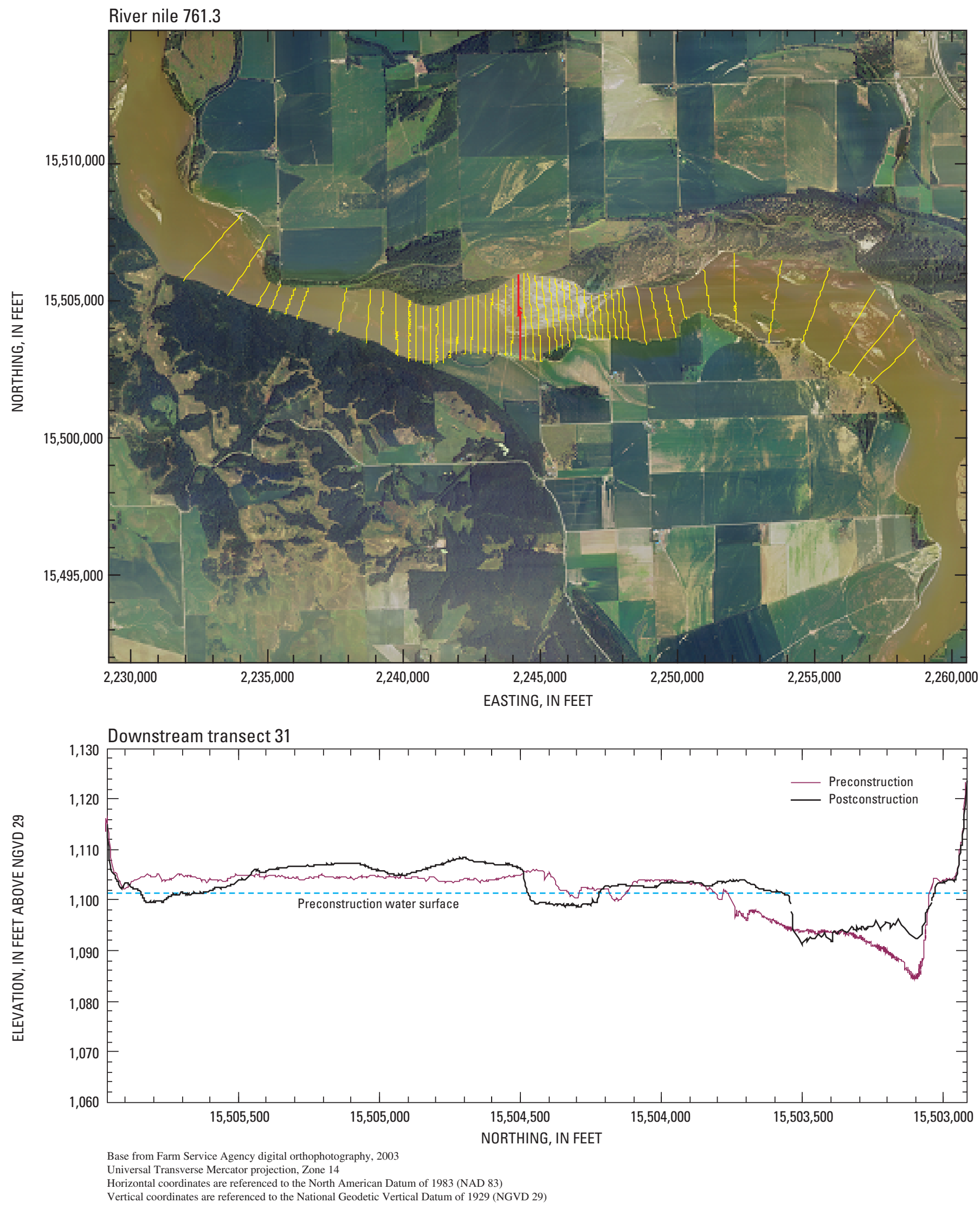

Figure A27. Location and cross section for downstream transect 31. 

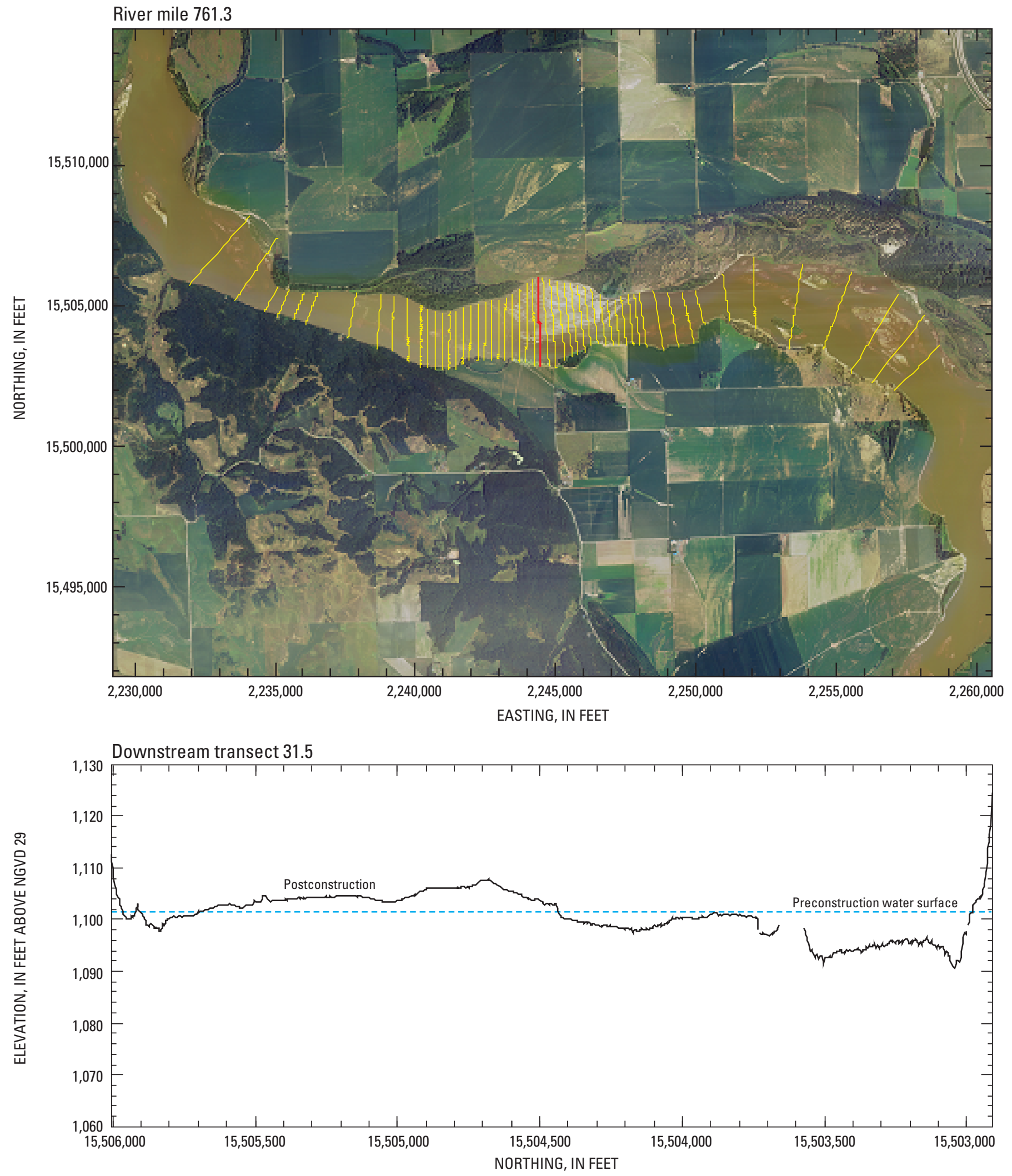

Base from Farm Service Agency digital orthophotography, 2003

Universal Transverse Mercator projection, Zone 14

Horizontal coordinates are referenced to the North American Datum of 1983 (NAD 83)

Vertical coordinates are referenced to the National Geodetic Vertical Datum of 1929 (NGVD 29)

Figure A28. Location and cross section for downstream transect 31.5. 

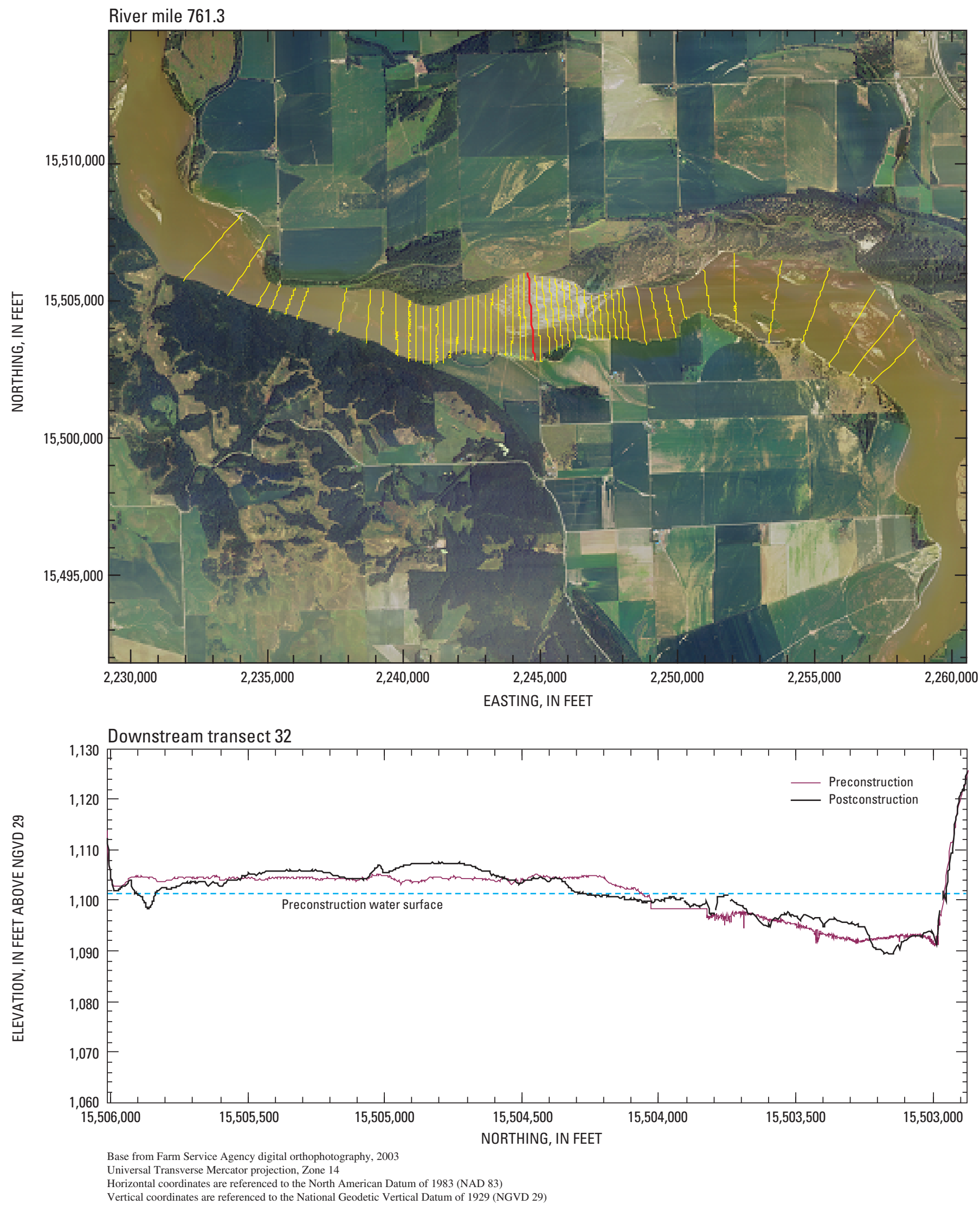

Figure A29. Location and cross section for downstream transect 32. 

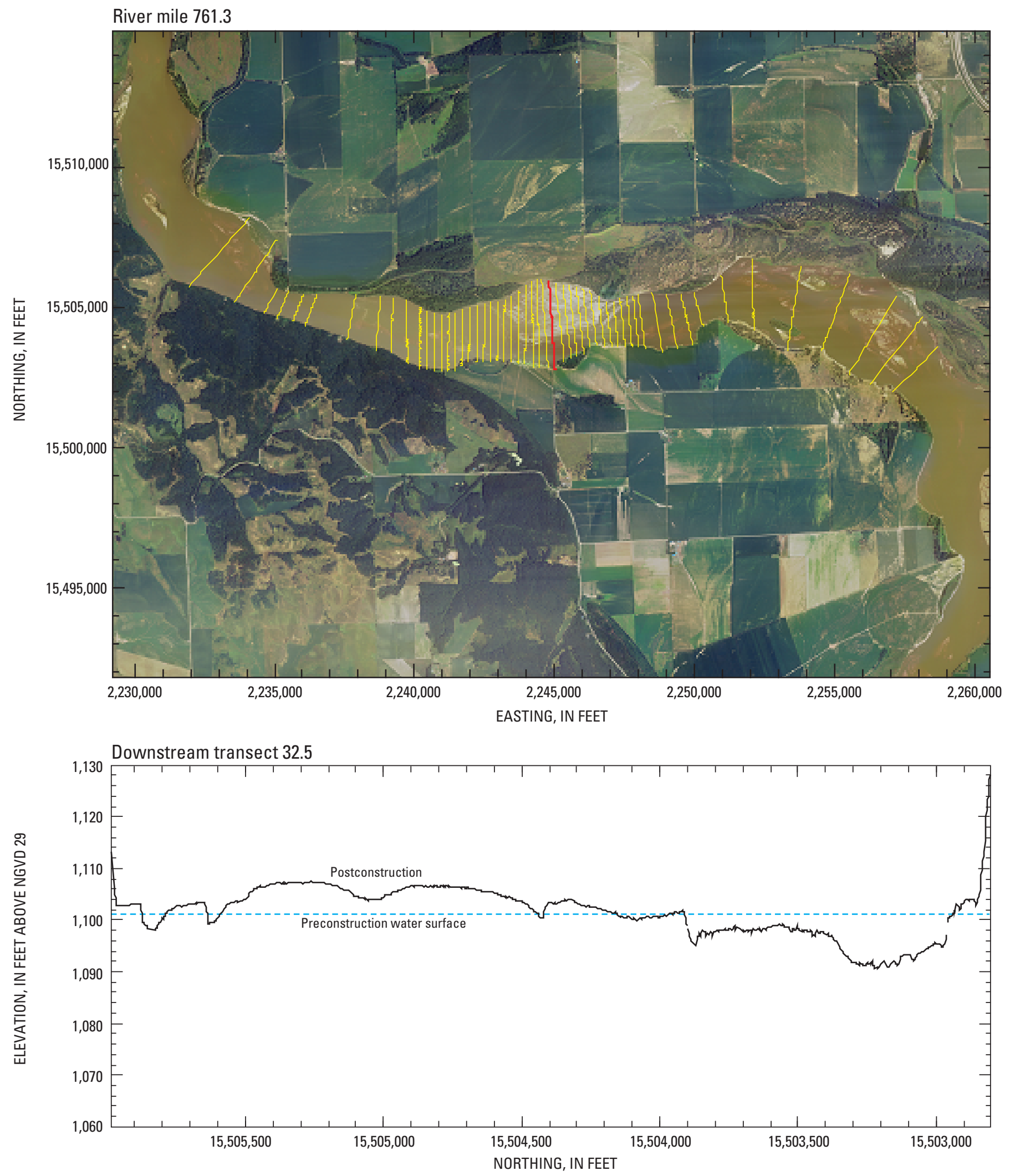

Base from Farm Service Agency digital orthophotography, 2003

Universal Transverse Mercator projection, Zone 14

Horizontal coordinates are referenced to the North American Datum of 1983 (NAD 83)

Vertical coordinates are referenced to the National Geodetic Vertical Datum of 1929 (NGVD 29)

Figure A30. Location and cross section for downstream transect 32.5. 

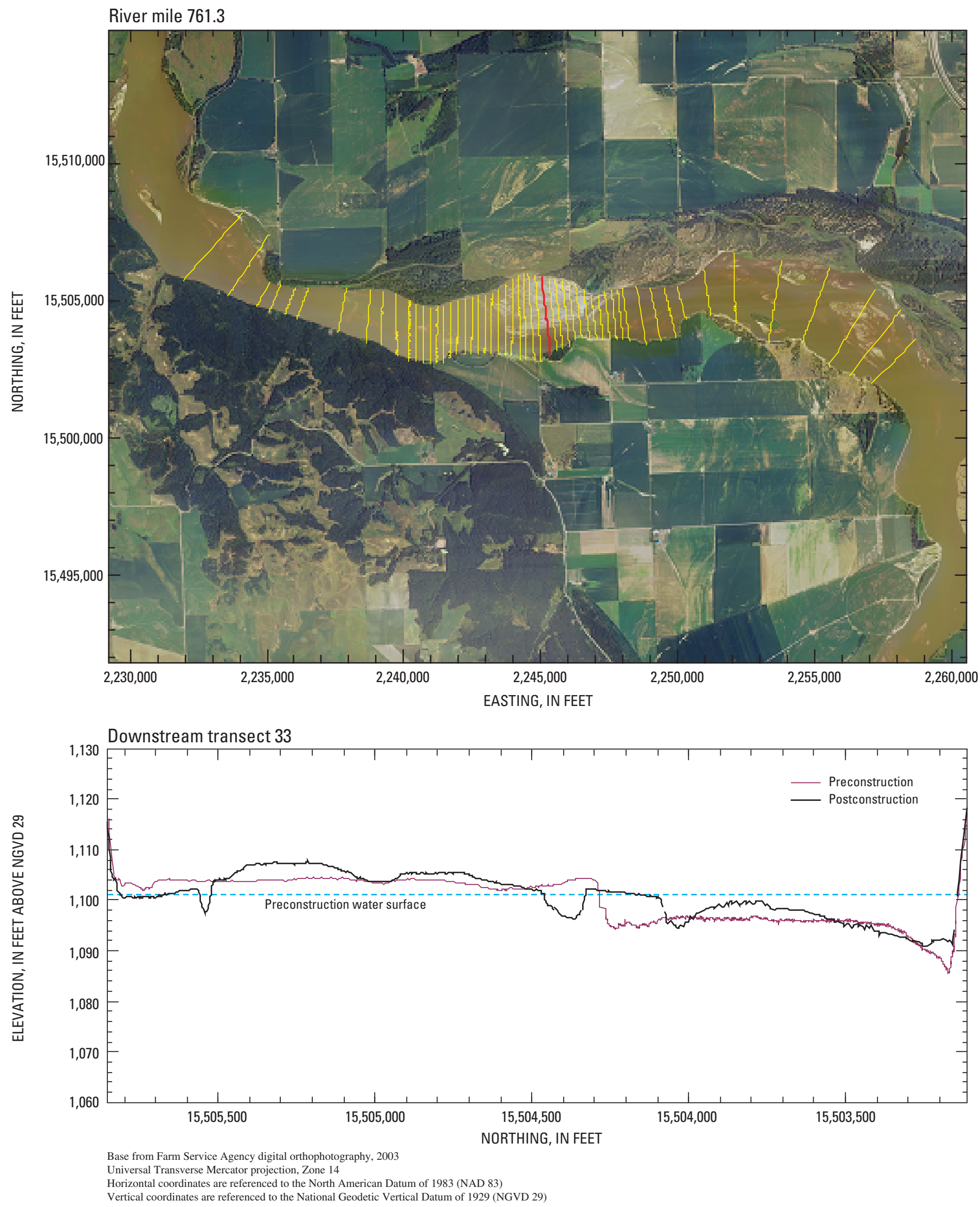

Figure A31. Location and cross section for downstream transect 33 . 

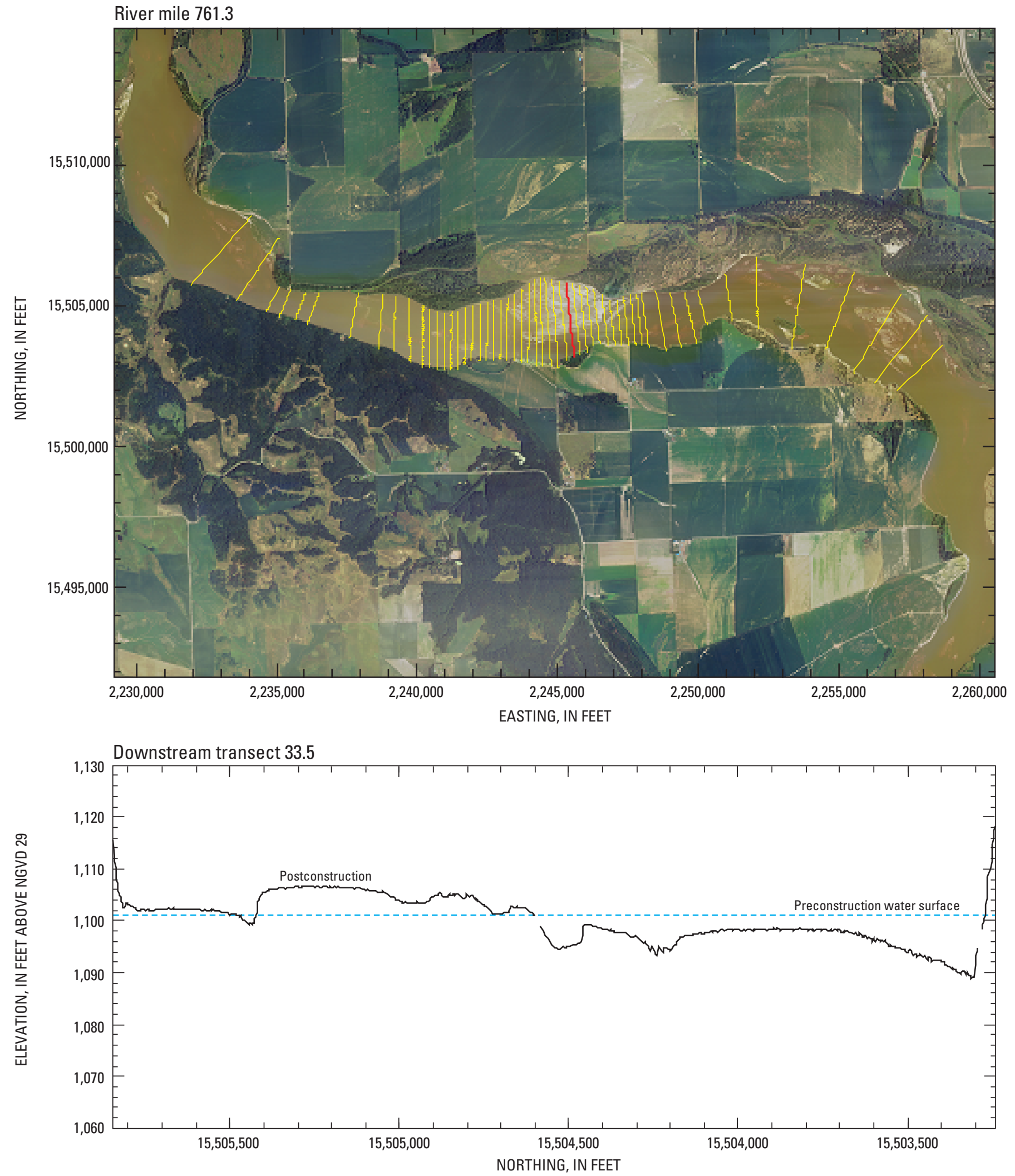

Base from Farm Service Agency digital orthophotography, 2003

Universal Transverse Mercator projection, Zone 14

Horizontal coordinates are referenced to the North American Datum of 1983 (NAD 83)

Vertical coordinates are referenced to the National Geodetic Vertical Datum of 1929 (NGVD 29)

Figure A32. Location and cross section for downstream transect 33.5. 

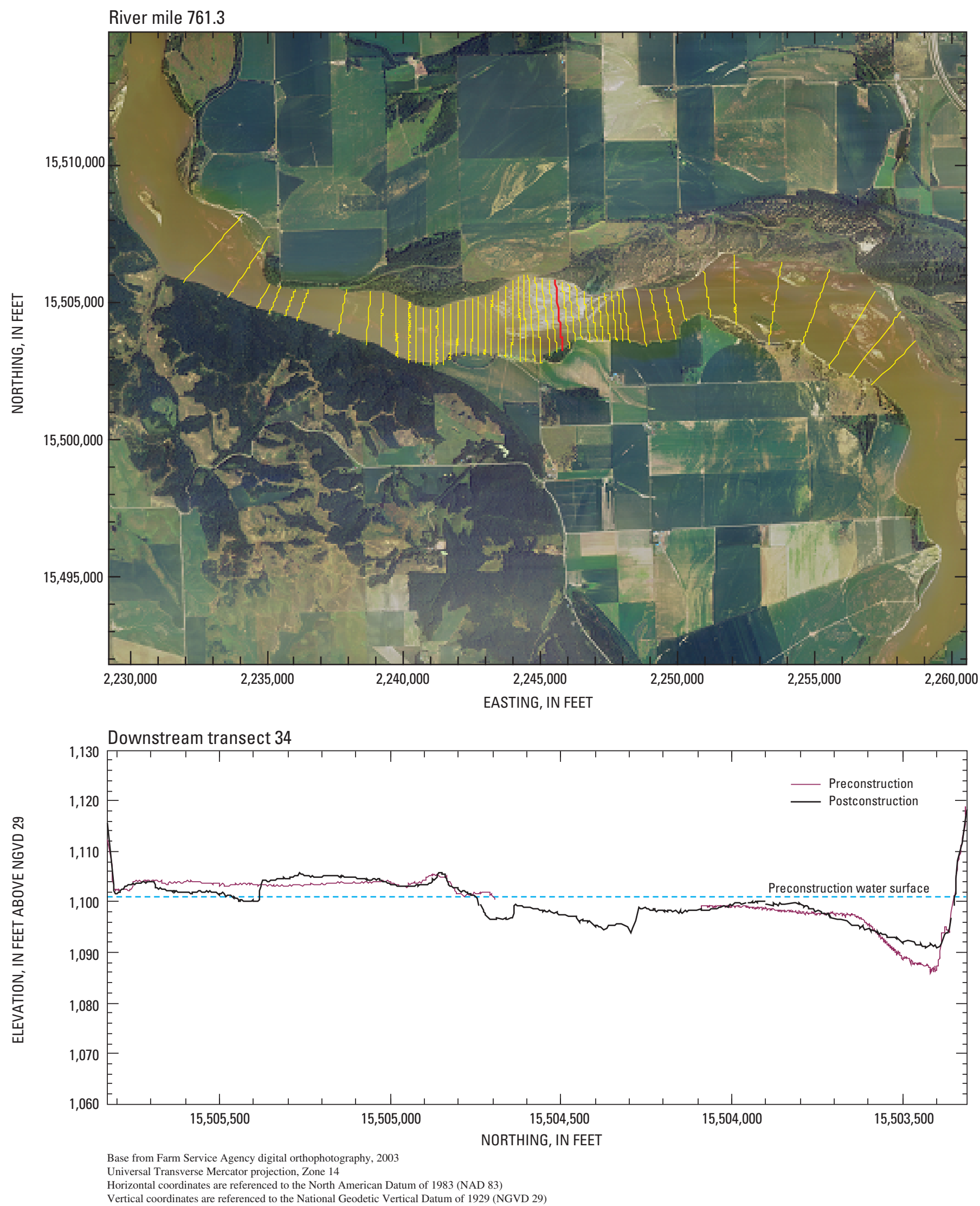

Figure A33. Location and cross section for downstream transect 34 . 

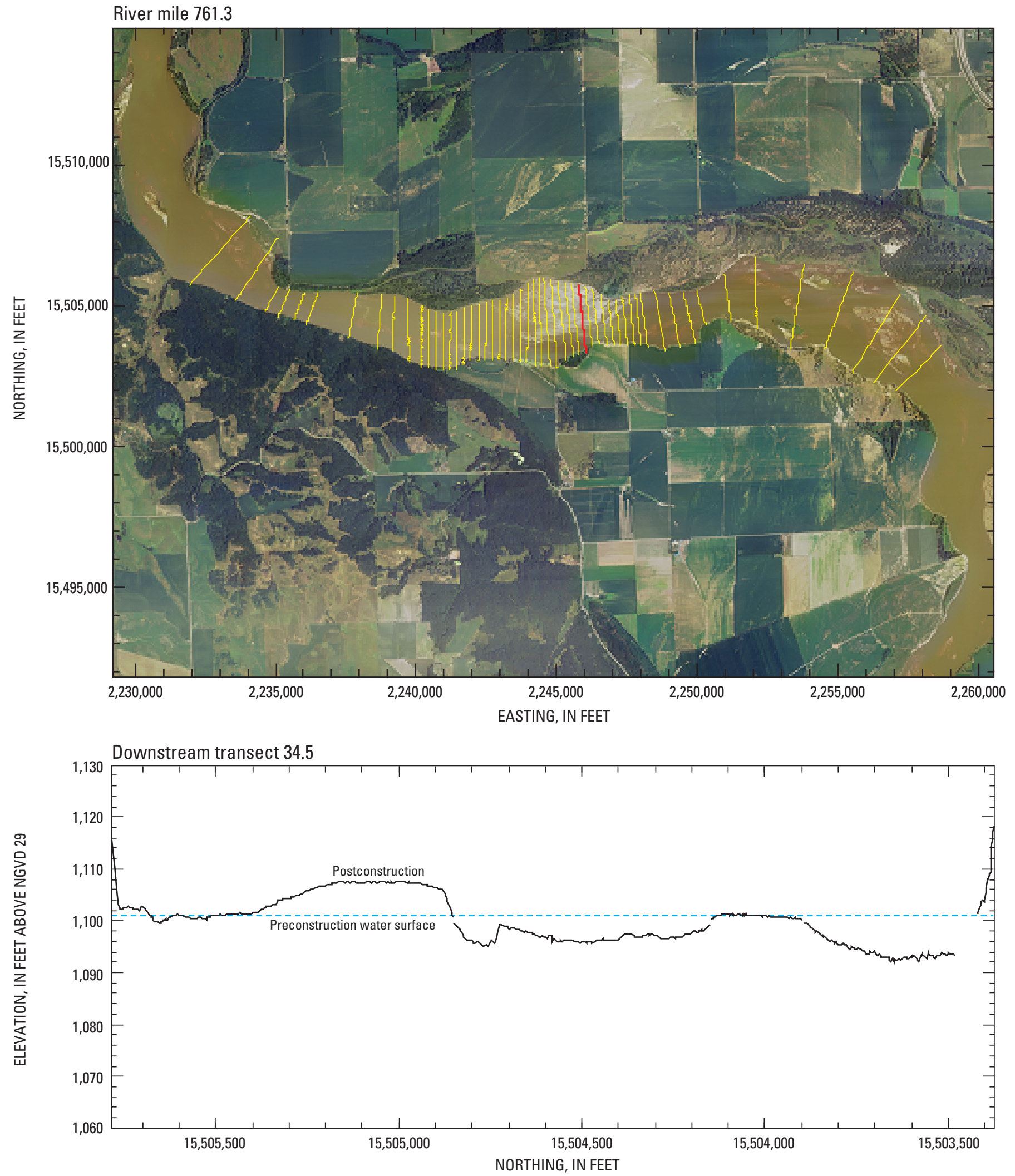

Base from Farm Service Agency digital orthophotography, 2003

Universal Transverse Mercator projection, Zone 14

Horizontal coordinates are referenced to the North American Datum of 1983 (NAD 83)

Vertical coordinates are referenced to the National Geodetic Vertical Datum of 1929 (NGVD 29)

Figure A34. Location and cross section for downstream transect 34.5. 

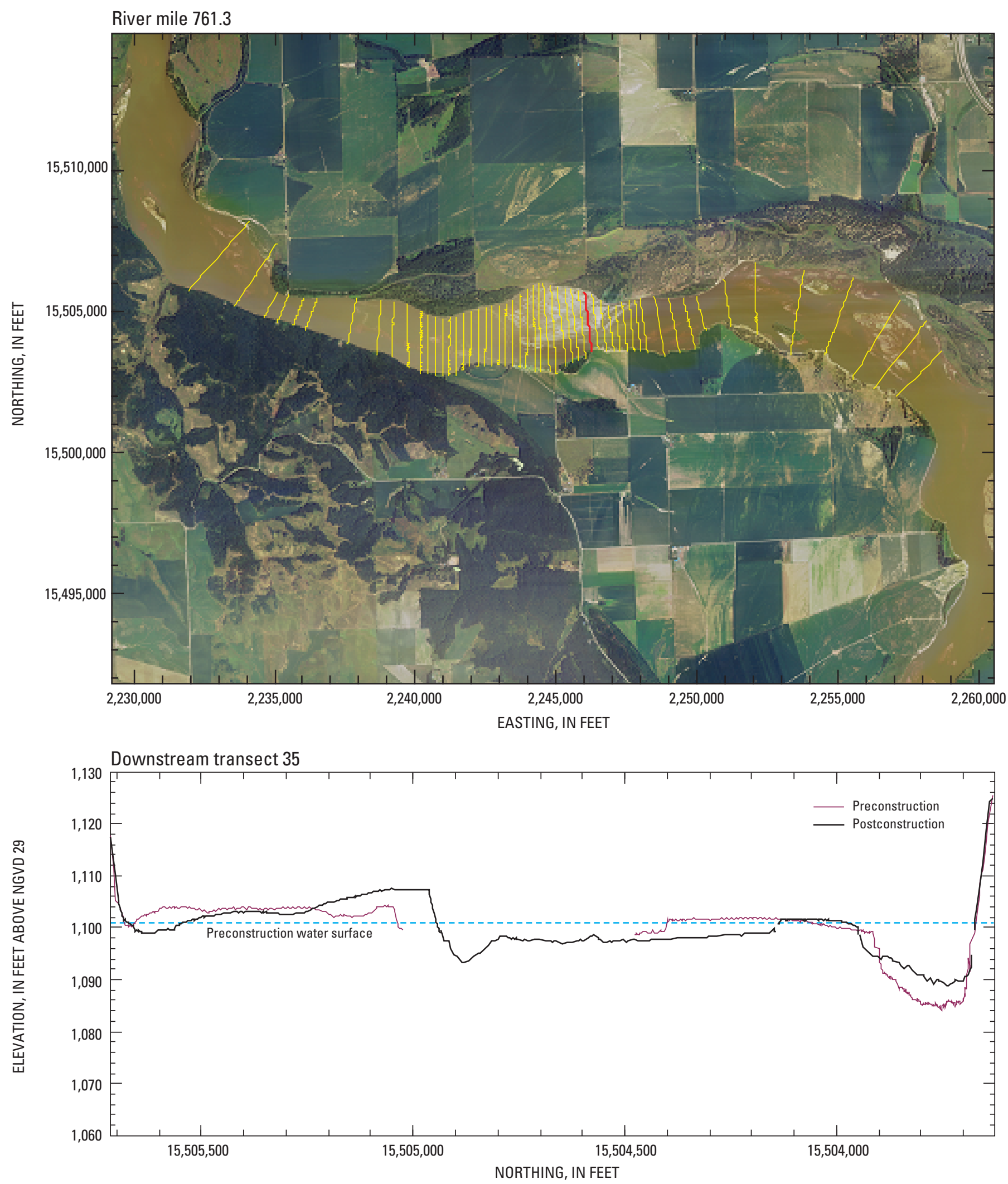

Base from Farm Service Agency digital orthophotography, 2003

Universal Transverse Mercator projection, Zone 14

Horizontal coordinates are referenced to the North American Datum of 1983 (NAD 83)

Vertical coordinates are referenced to the National Geodetic Vertical Datum of 1929 (NGVD 29)

Figure A35. Location and cross section for downstream transect 35 . 

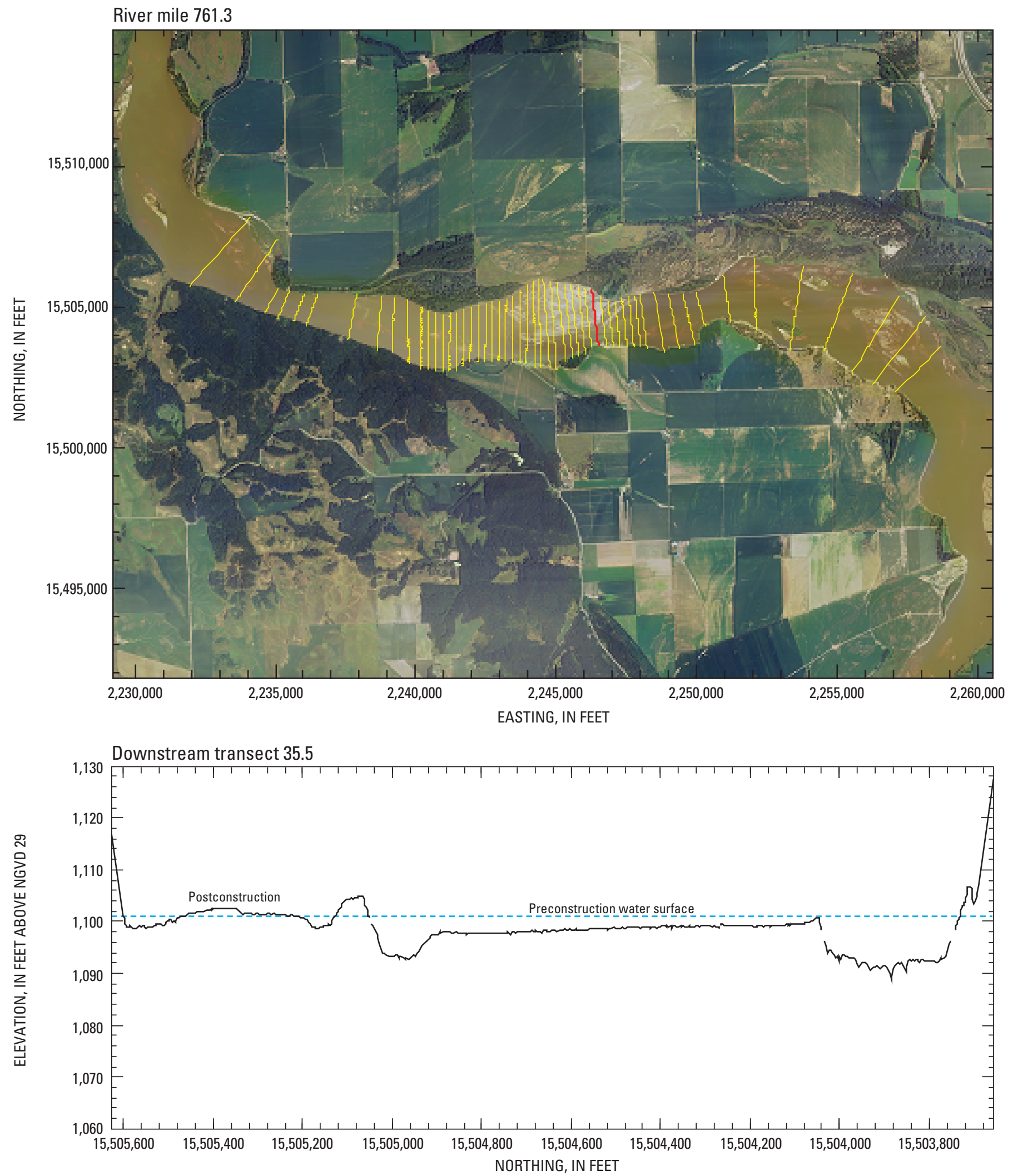

Base from Farm Service Agency digital orthophotography, 2003

Universal Transverse Mercator projection, Zone 14

Horizontal coordinates are referenced to the North American Datum of 1983 (NAD 83)

Vertical coordinates are referenced to the National Geodetic Vertical Datum of 1929 (NGVD 29)

Figure A36. Location and cross section for downstream transect 35.5. 

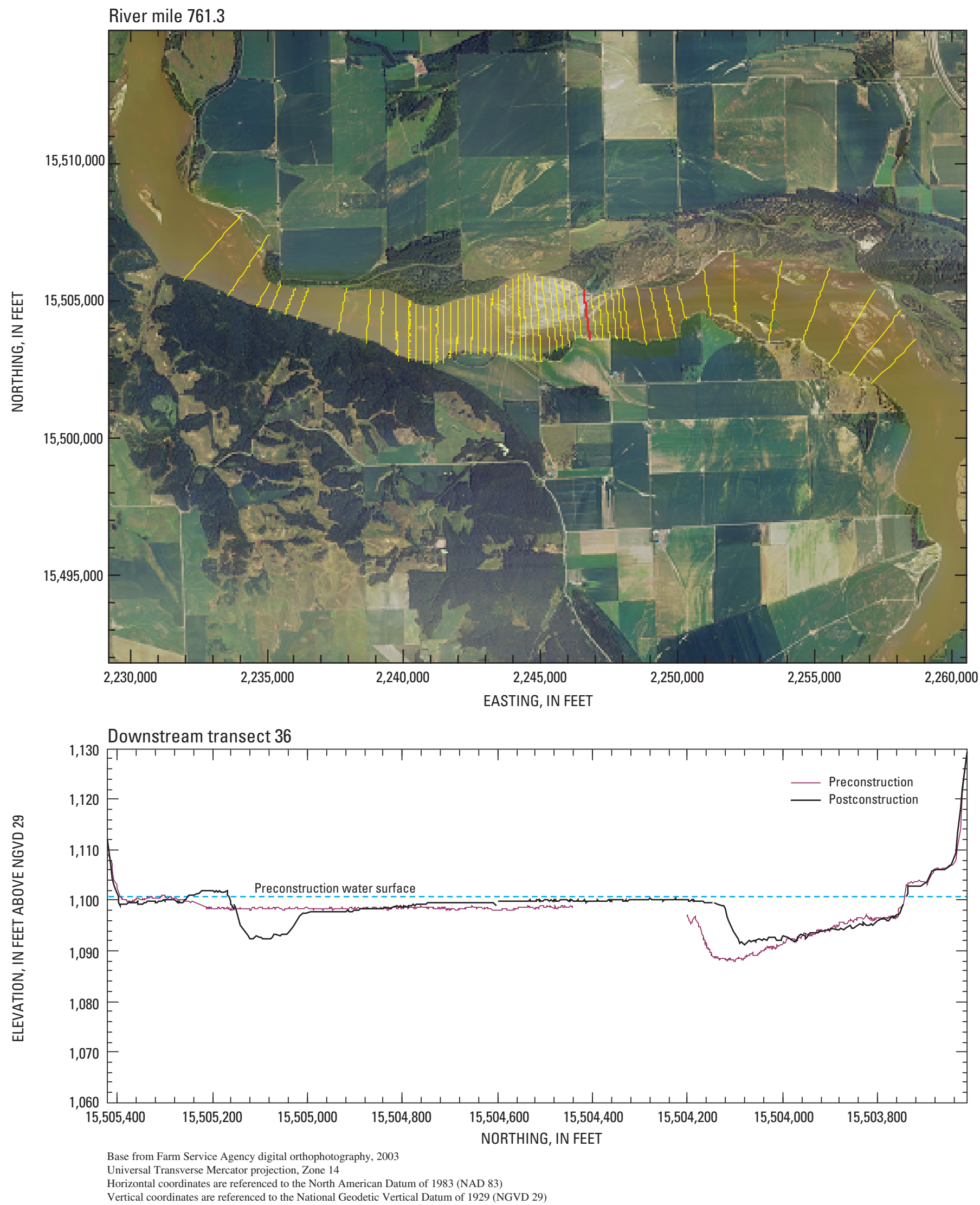

Figure A37. Location and cross section for downstream transect 36 . 

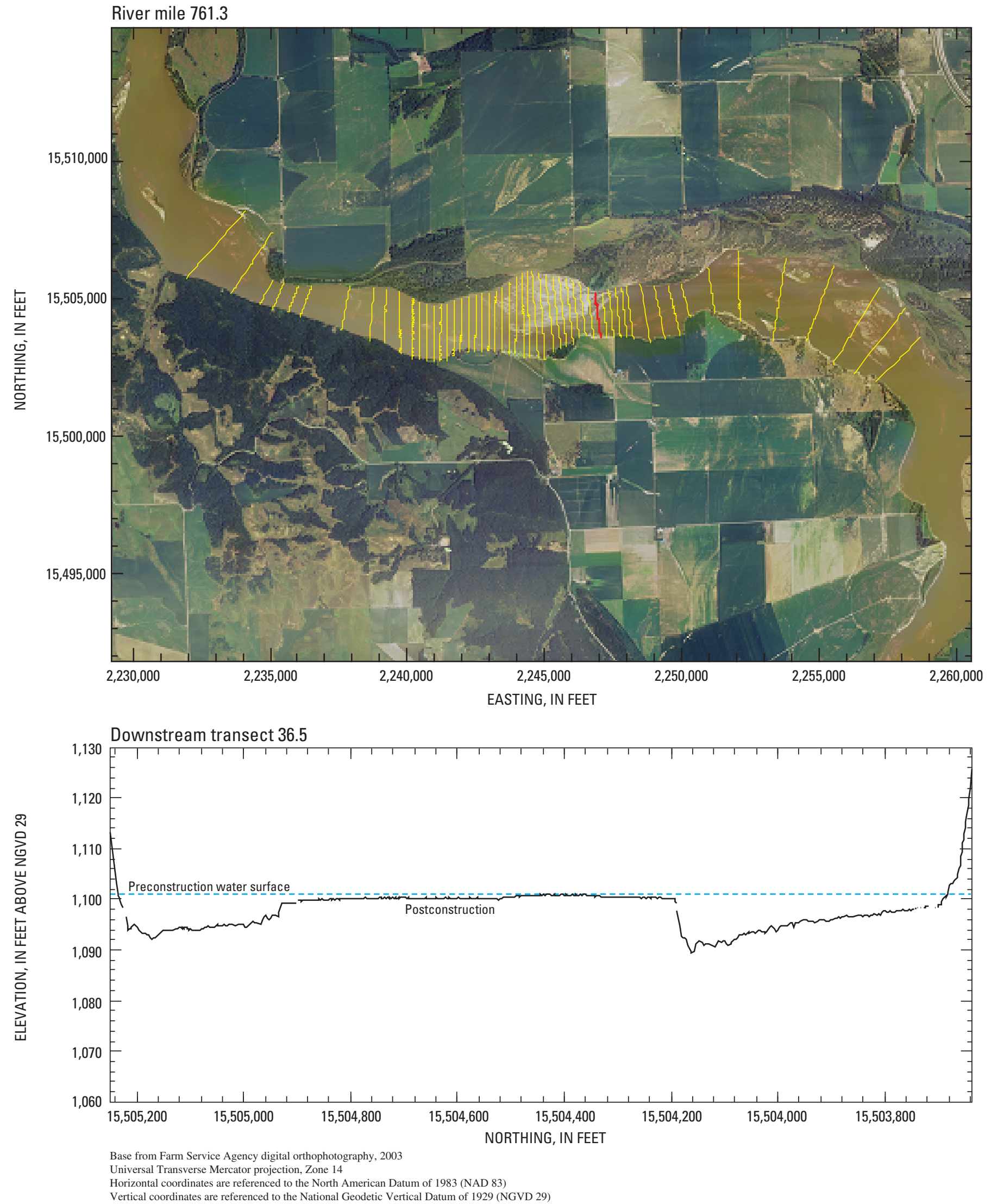

Figure A38. Location and cross section for downstream transect 36.5. 

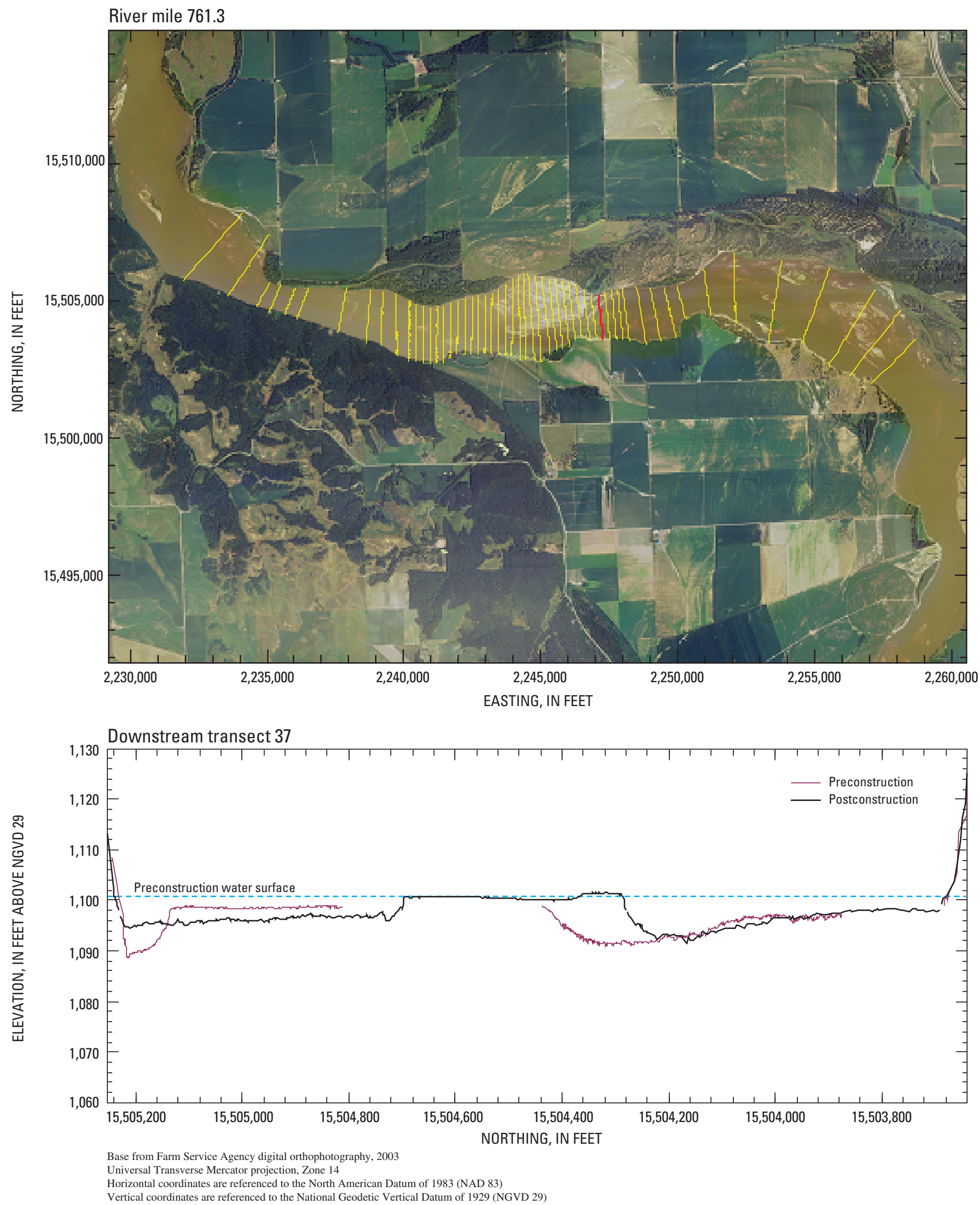

Figure A39. Location and cross section for downstream transect 37 . 

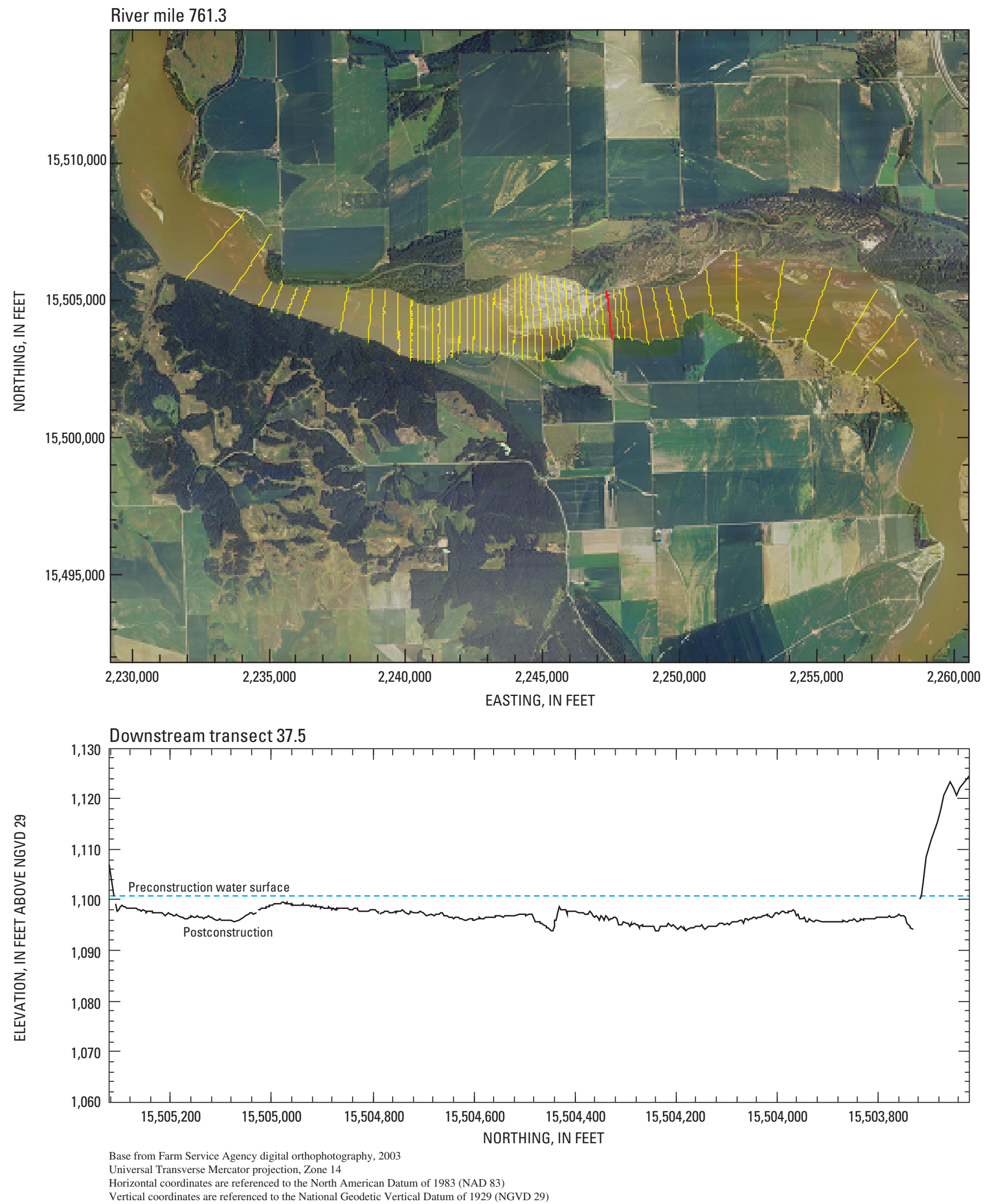

Figure A40. Location and cross section for downstream transect 37.5. 

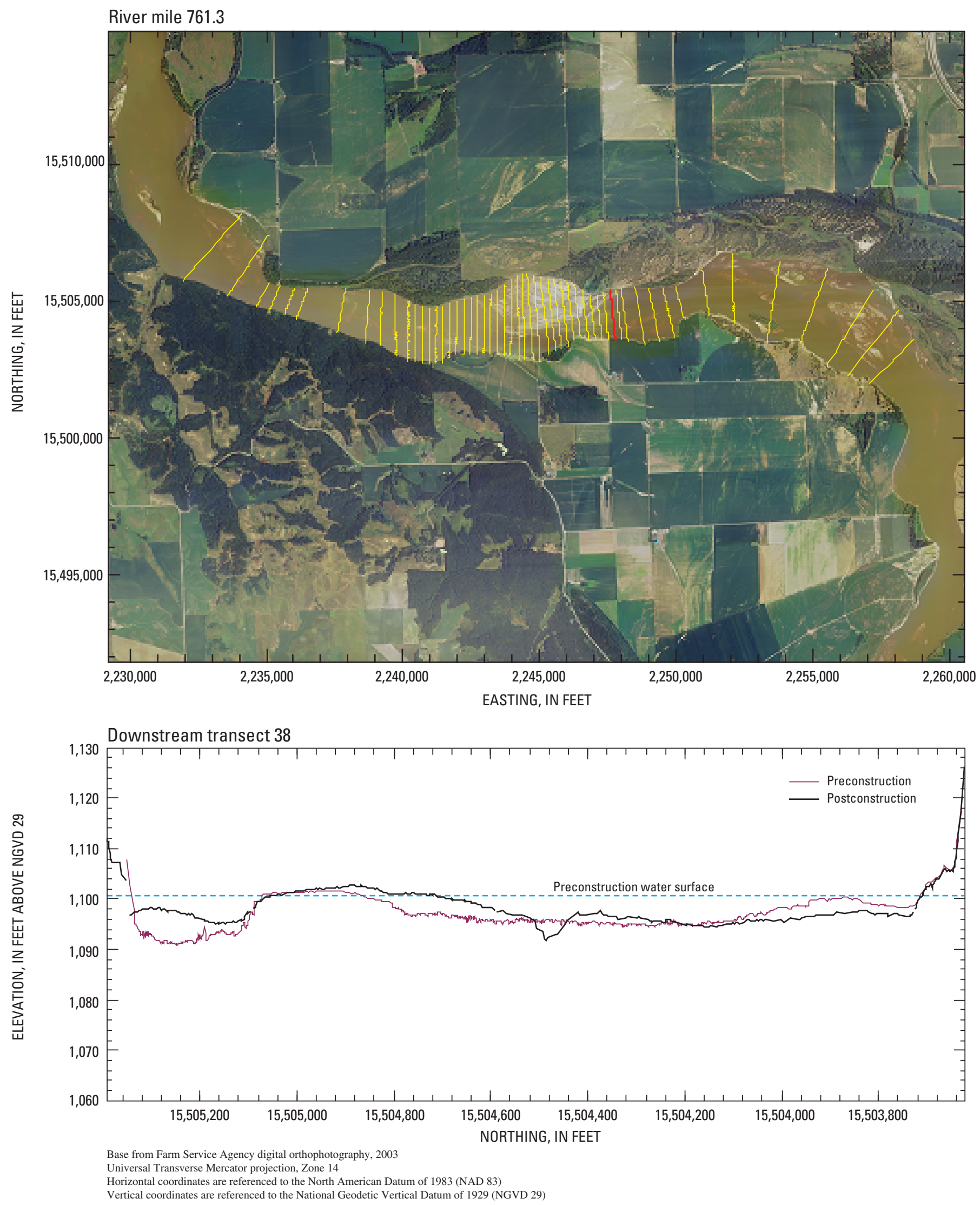

Figure A41. Location and cross section for downstream transect 38. 

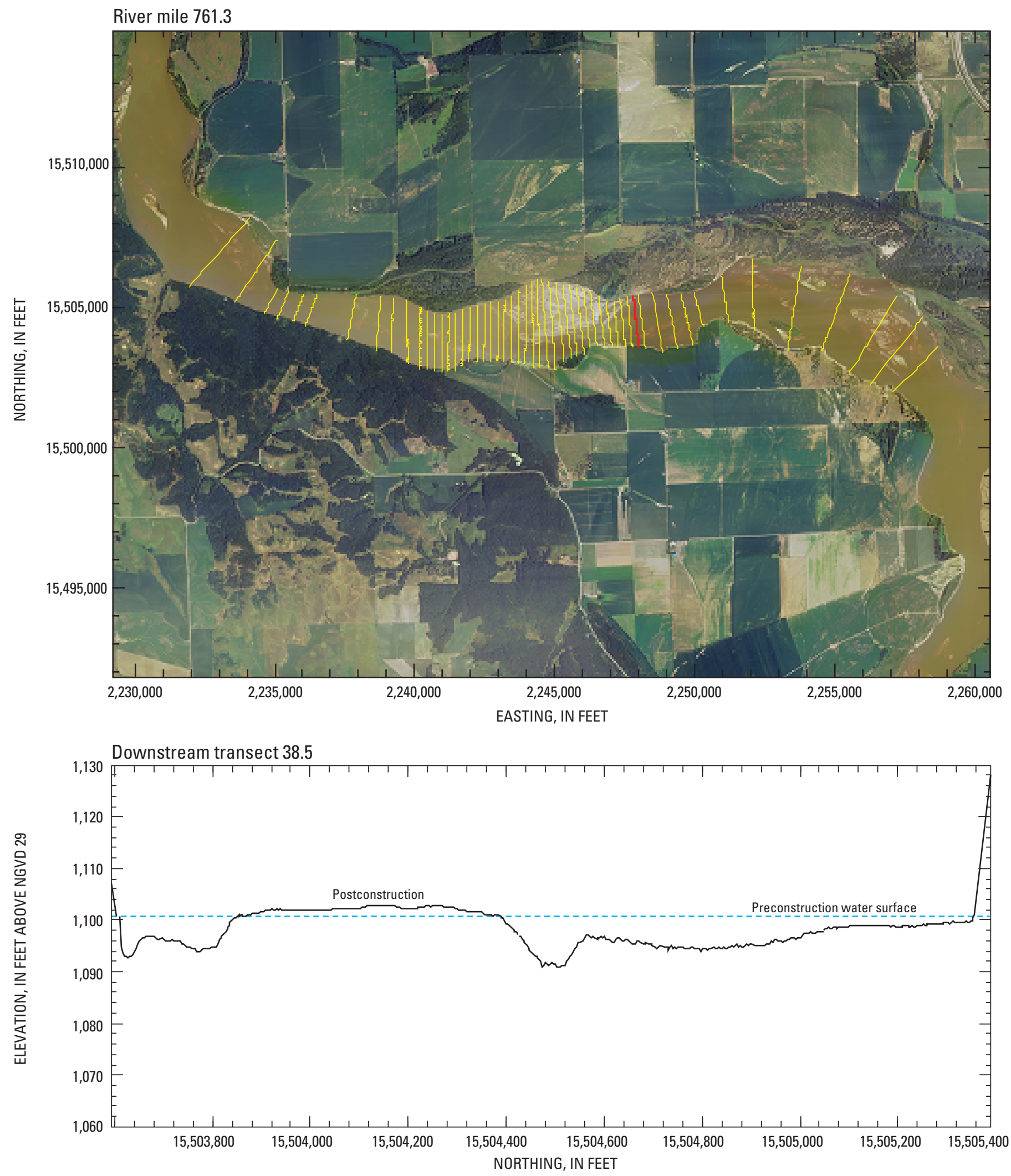

Base from Farm Service Agency digital orthophotography, 2003

Universal Transverse Mercator projection, Zone 14

Horizontal coordinates are referenced to the North American Datum of 1983 (NAD 83)

Vertical coordinates are referenced to the National Geodetic Vertical Datum of 1929 (NGVD 29)

Figure A42. Location and cross section for downstream transect 38.5. 

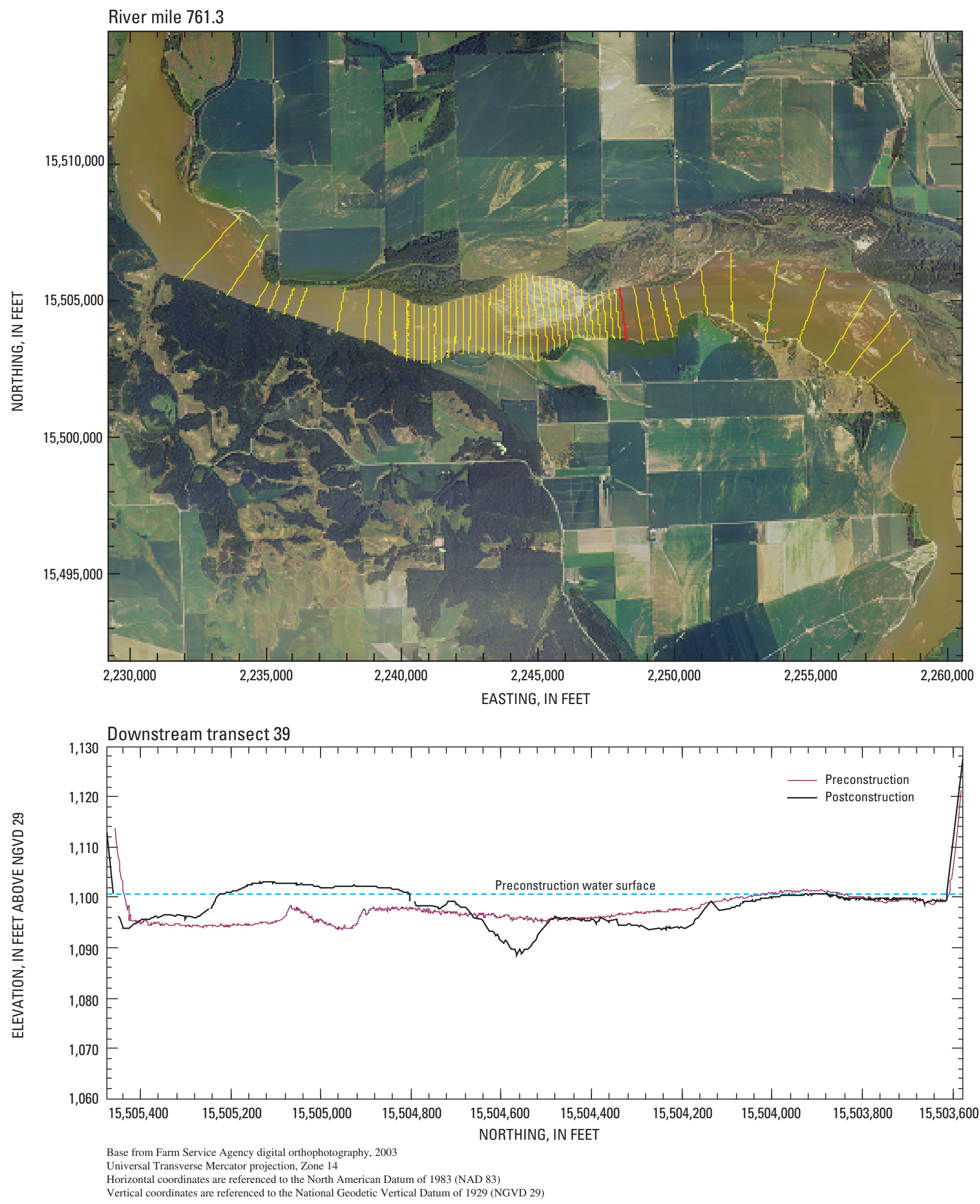

Figure A43. Location and cross section for downstream transect 39 . 

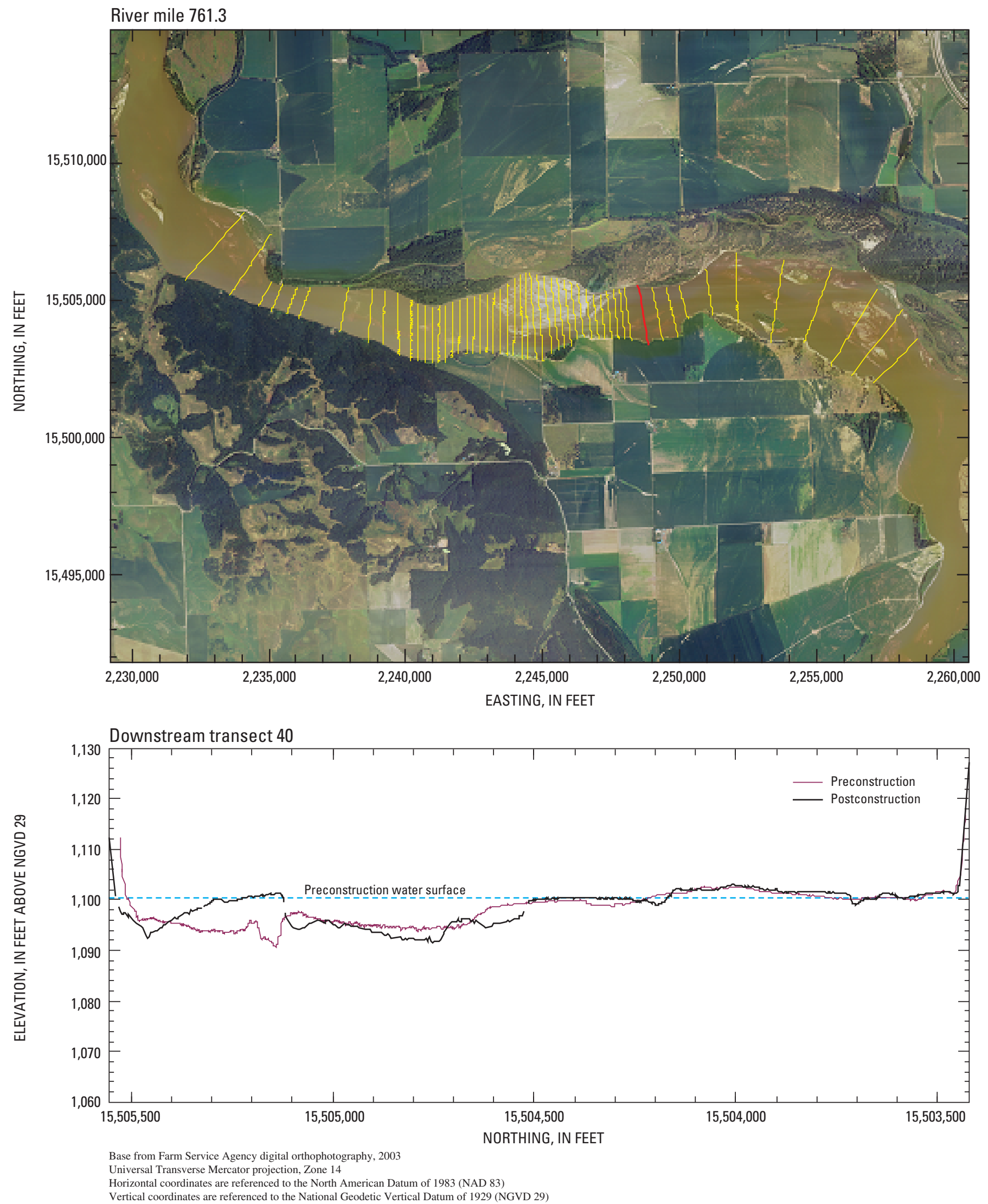

Figure A44. Location and cross section for downstream transect 40. 

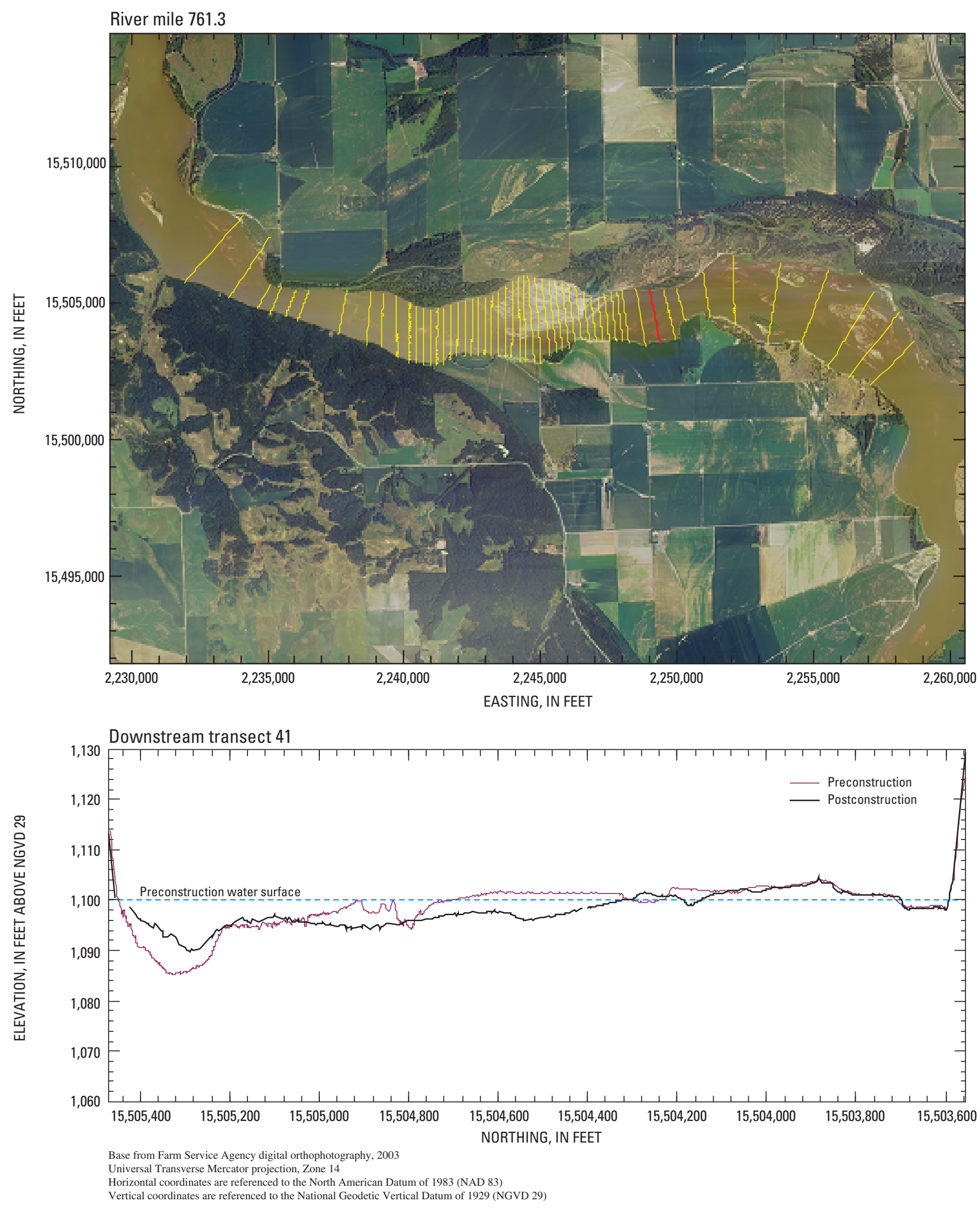

Figure A45. Location and cross section for downstream transect 41. 

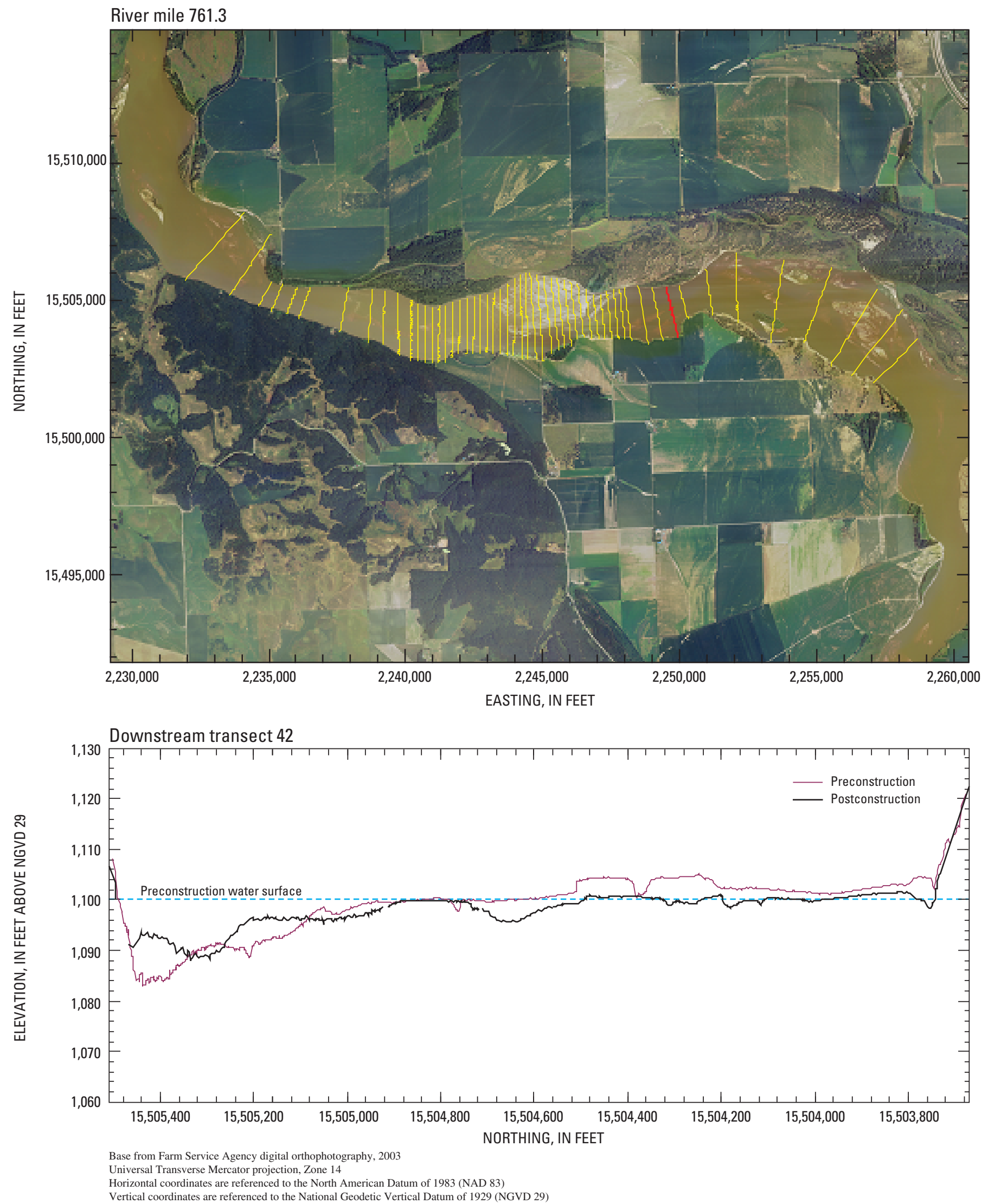

Figure A46. Location and cross section for downstream transect 42. 

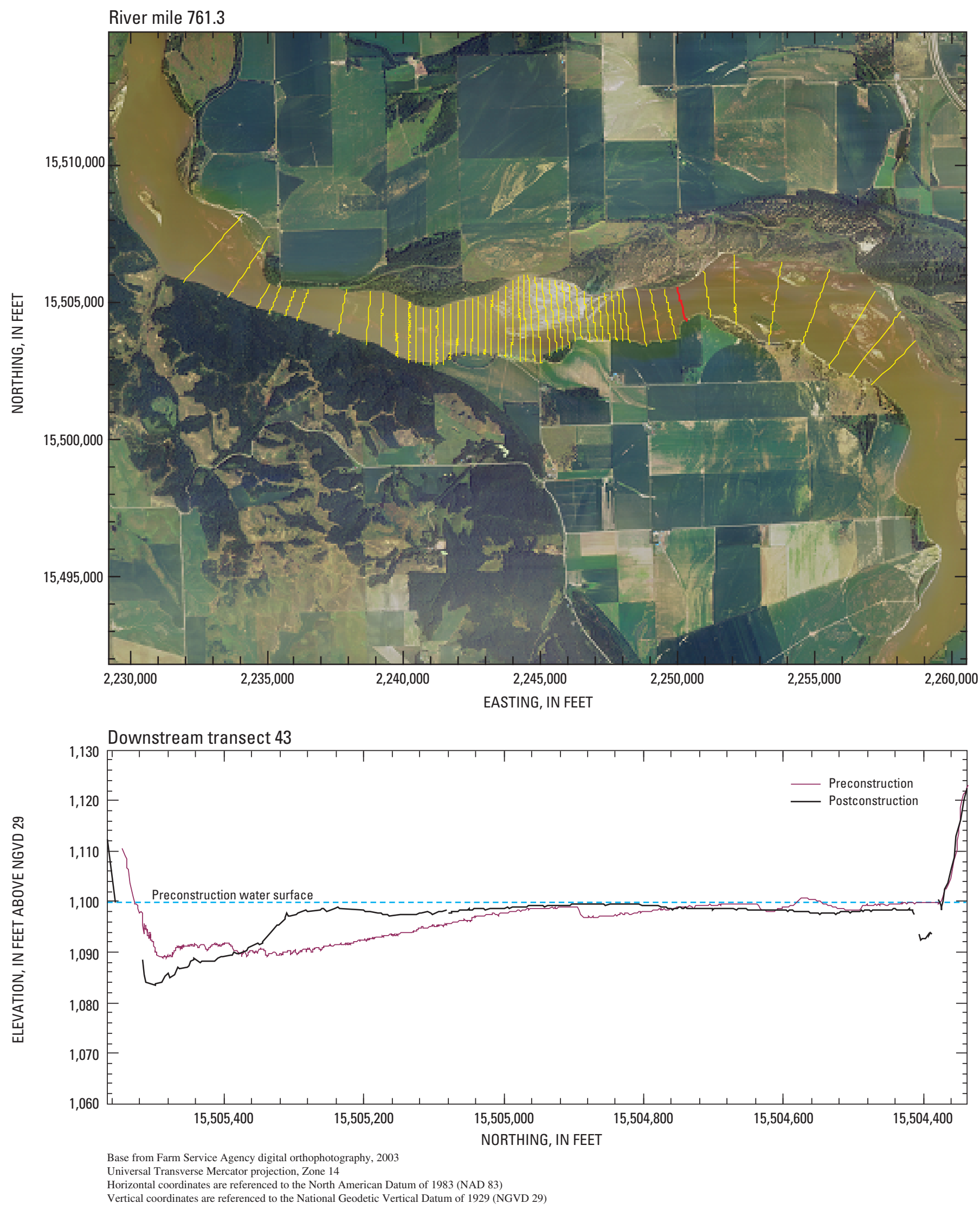

Figure A47. Location and cross section for downstream transect 43. 

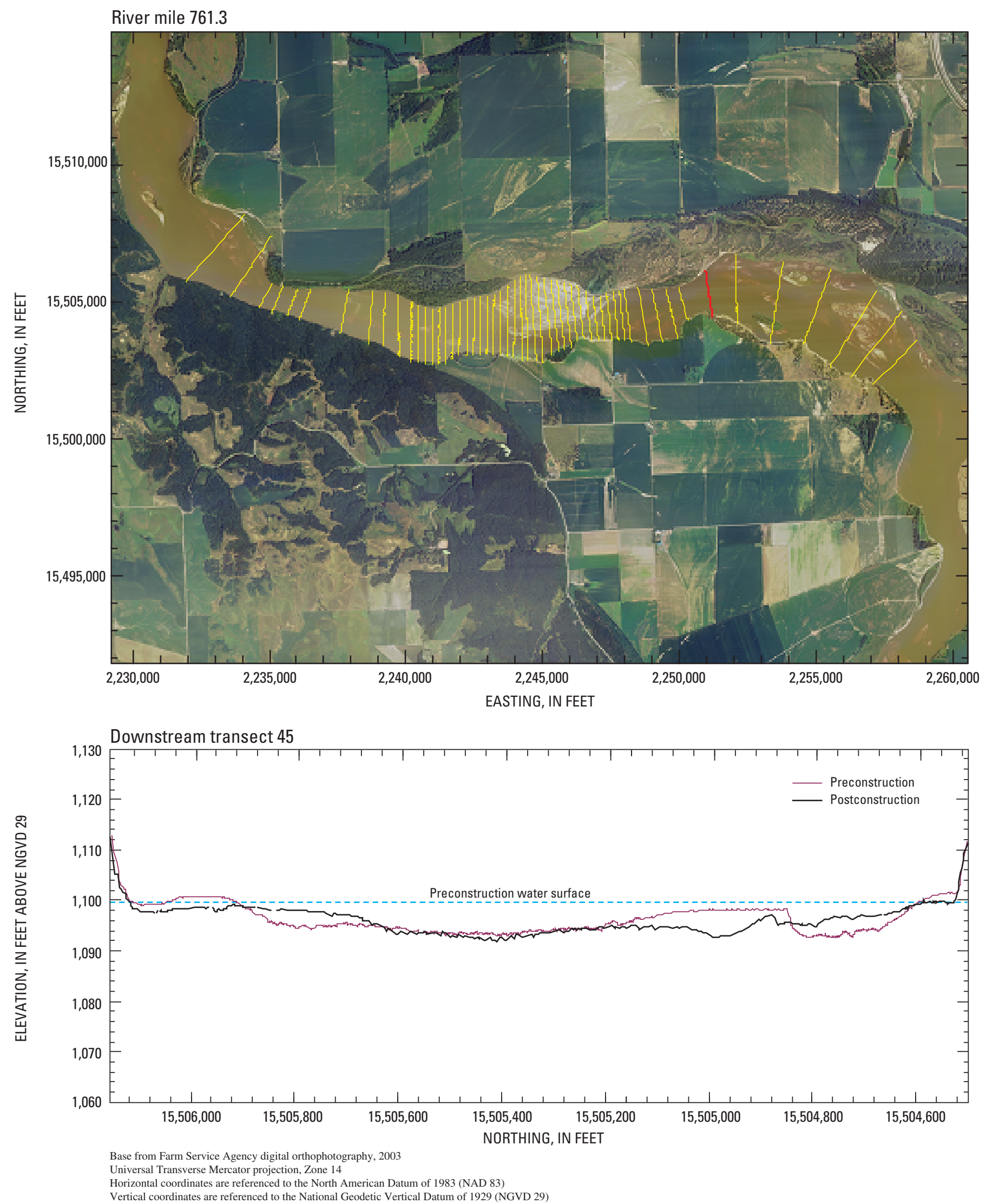

Figure A48. Location and cross section for downstream transect 45 . 

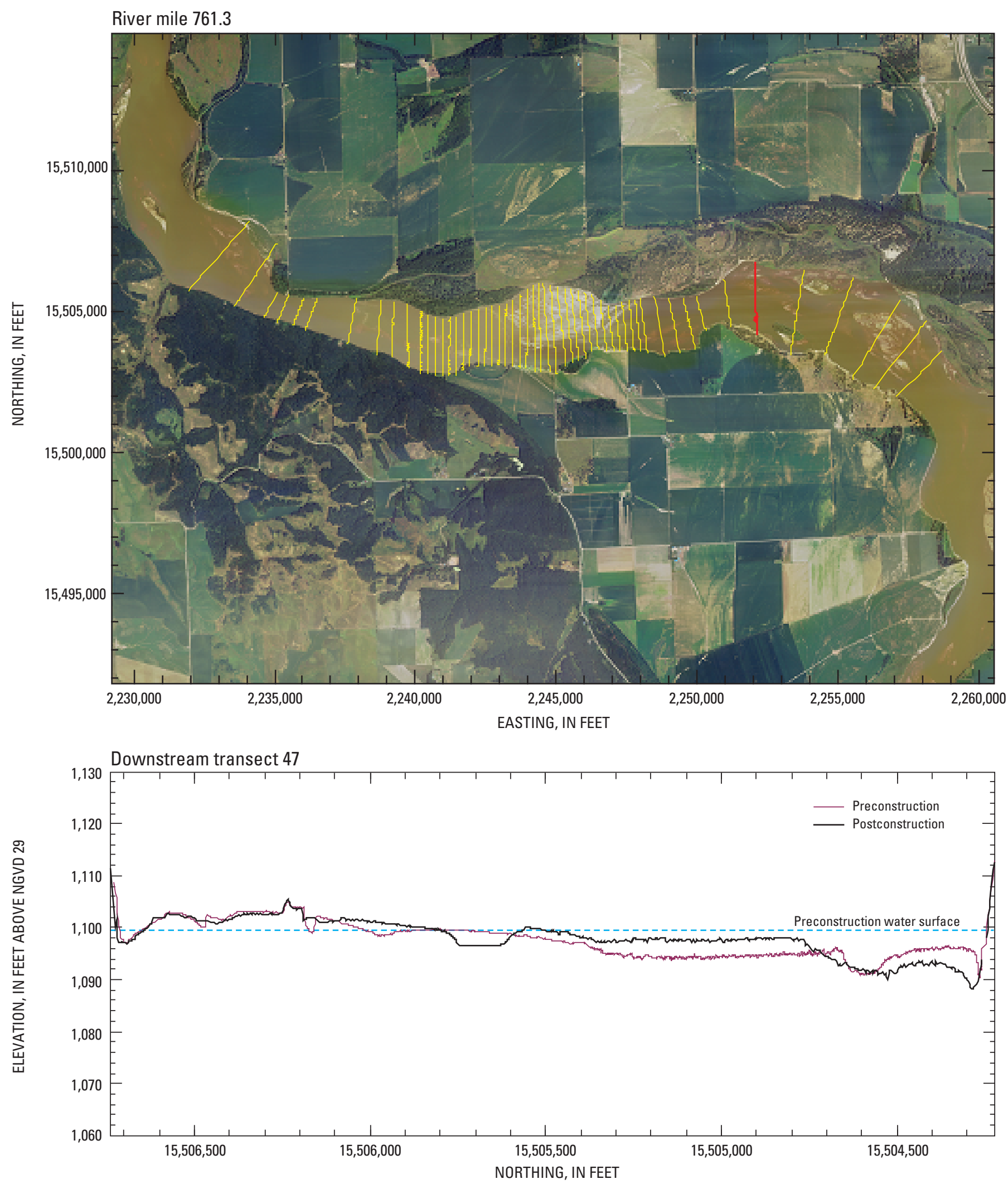

Base from Farm Service Agency digital orthophotography, 2003

Universal Transverse Mercator projection, Zone 14

Horizontal coordinates are referenced to the North American Datum of 1983 (NAD 83)

Vertical coordinates are referenced to the National Geodetic Vertical Datum of 1929 (NGVD 29)

Figure A49. Location and cross section for downstream transect 47. 

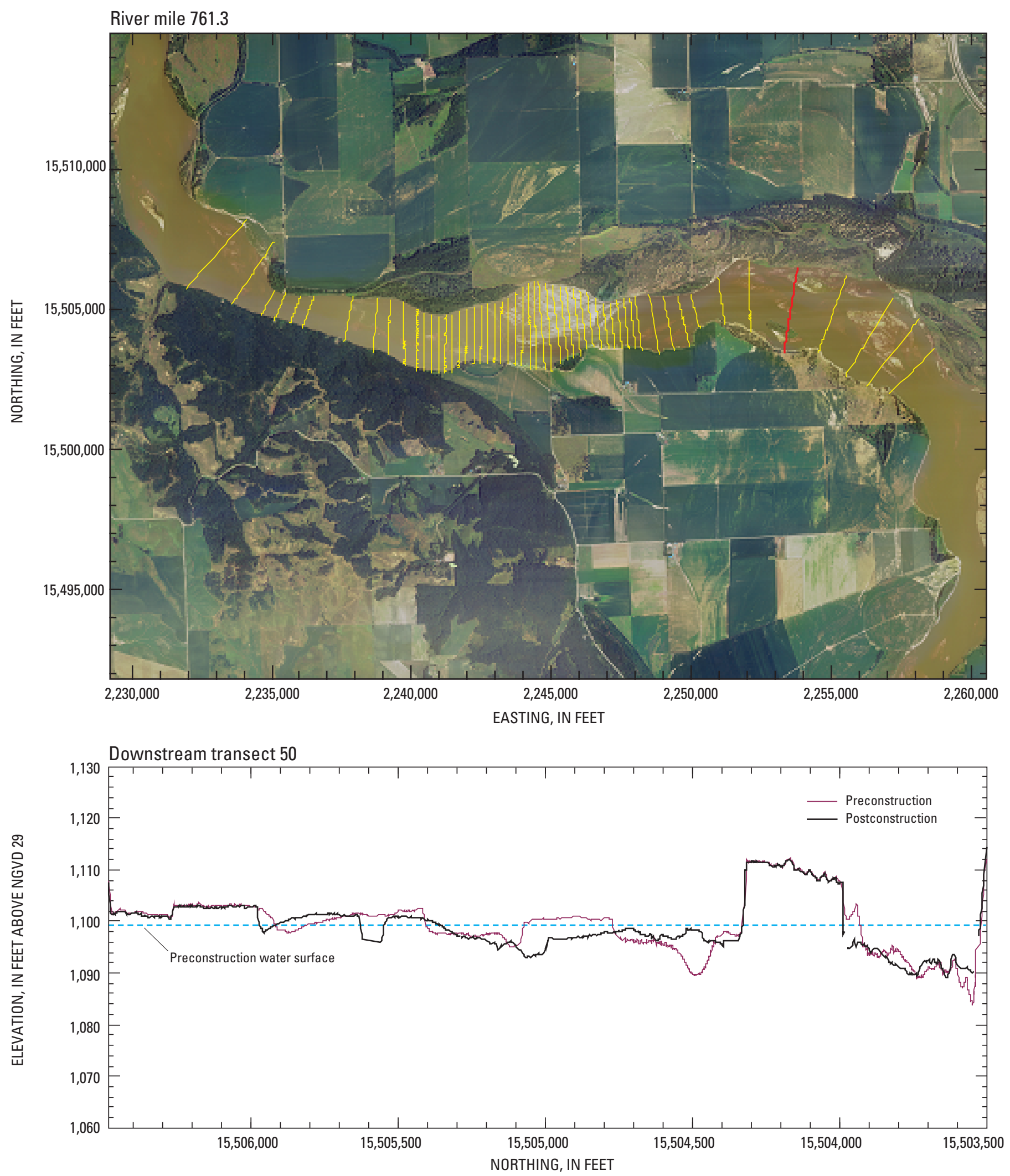

Base from Farm Service Agency digital orthophotography, 2003

Universal Transverse Mercator projection, Zone 14

Horizontal coordinates are referenced to the North American Datum of 1983 (NAD 83)

Vertical coordinates are referenced to the National Geodetic Vertical Datum of 1929 (NGVD 29)

Figure A50. Location and cross section for downstream transect 50. 

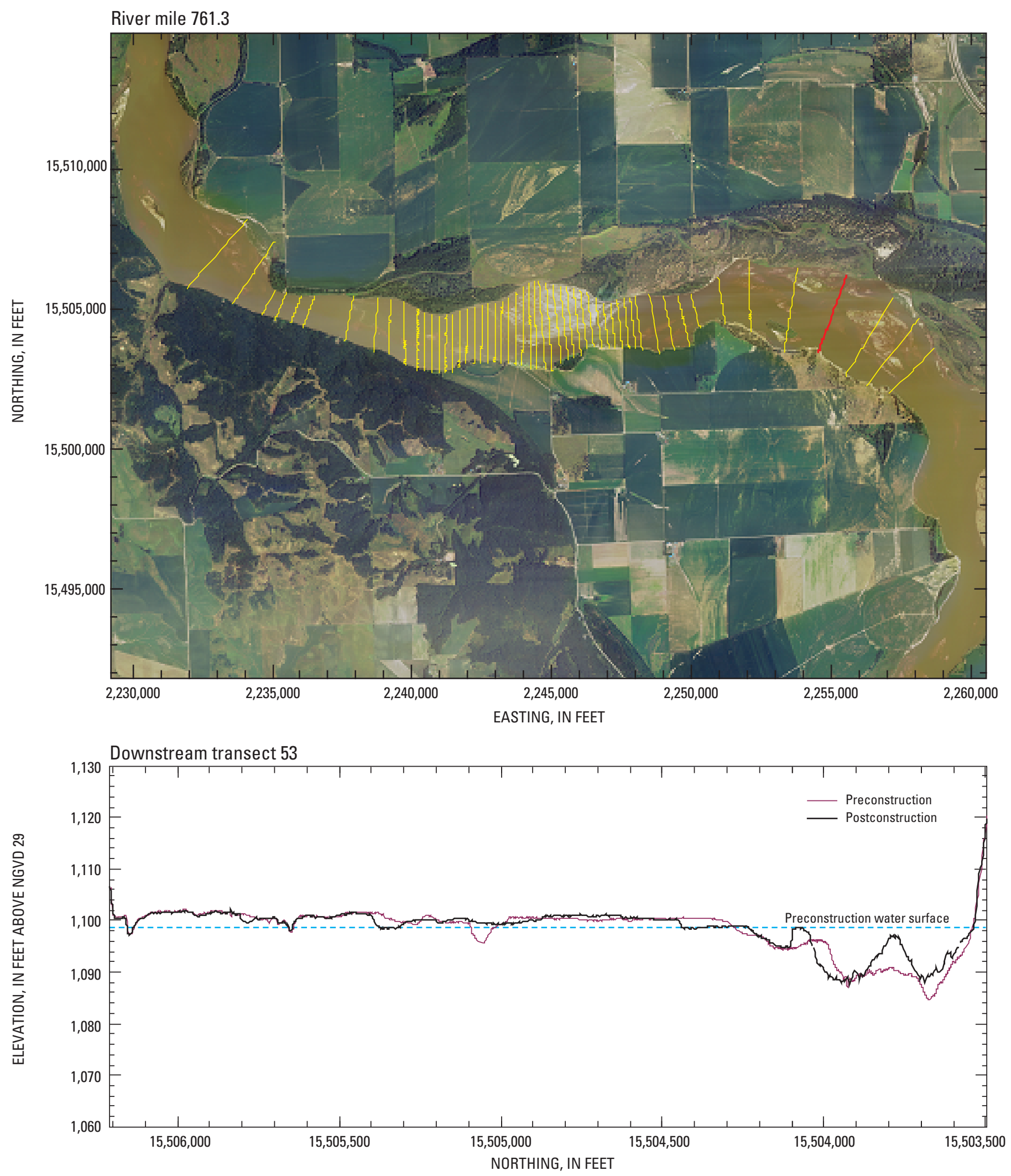

Base from Farm Service Agency digital orthophotography, 2003

Universal Transverse Mercator projection, Zone 14

Horizontal coordinates are referenced to the North American Datum of 1983 (NAD 83)

Vertical coordinates are referenced to the National Geodetic Vertical Datum of 1929 (NGVD 29)

Figure A51. Location and cross section for downstream transect 53. 

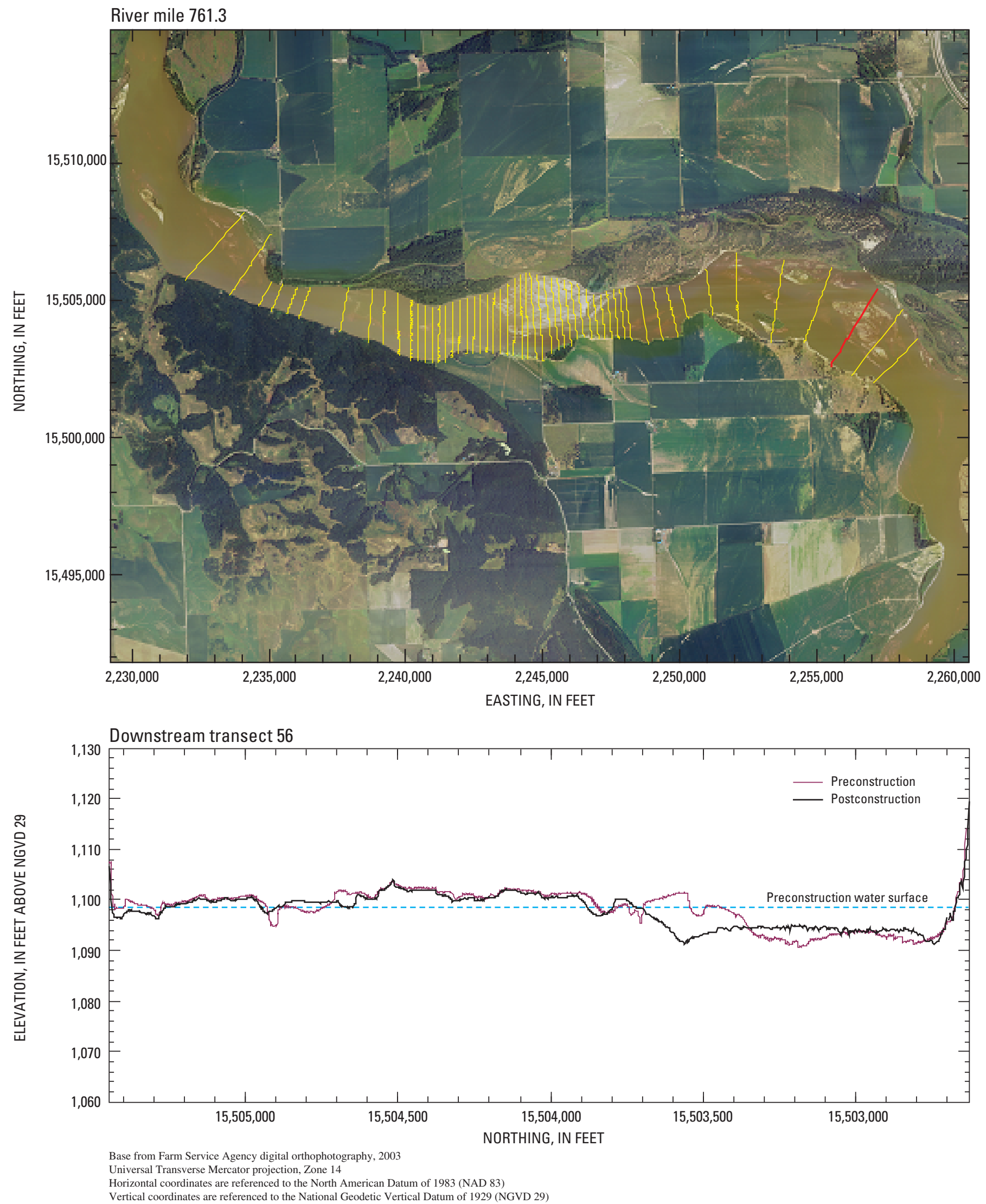

Figure A52. Location and cross section for downstream transect 56 . 

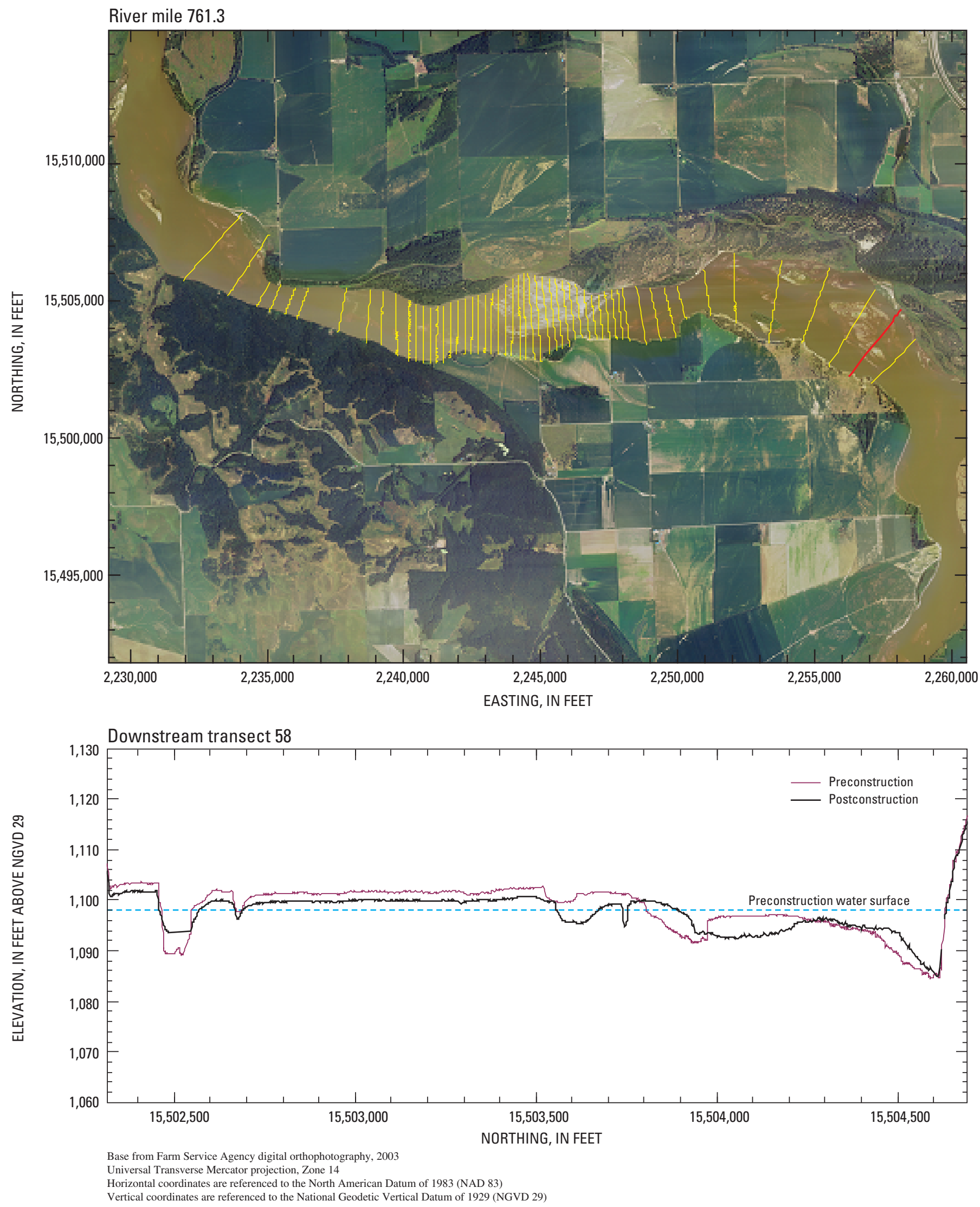

Figure A53. Location and cross section for downstream transect 58 . 

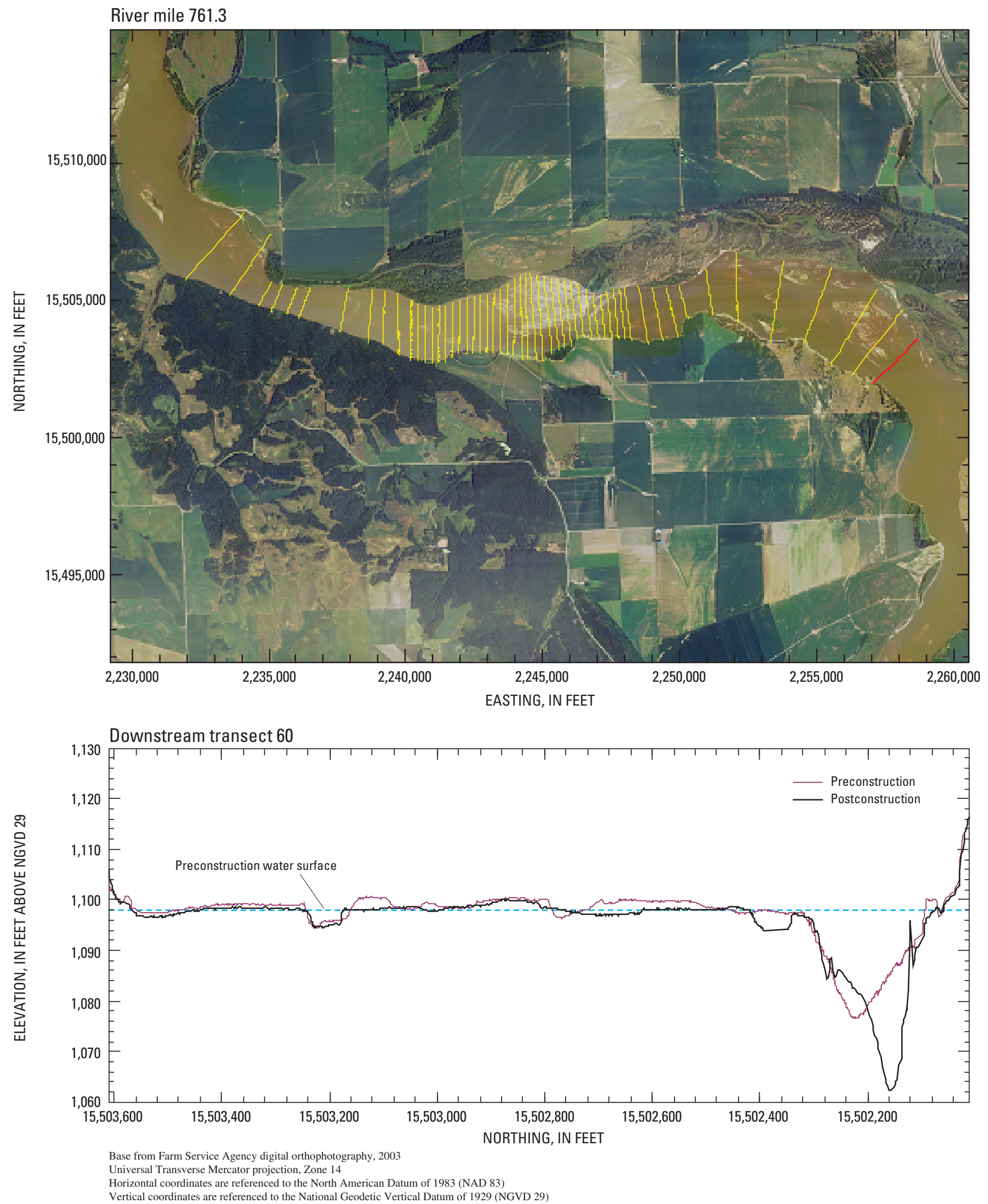

Figure A54. Location and cross section for downstream transect 60 . 

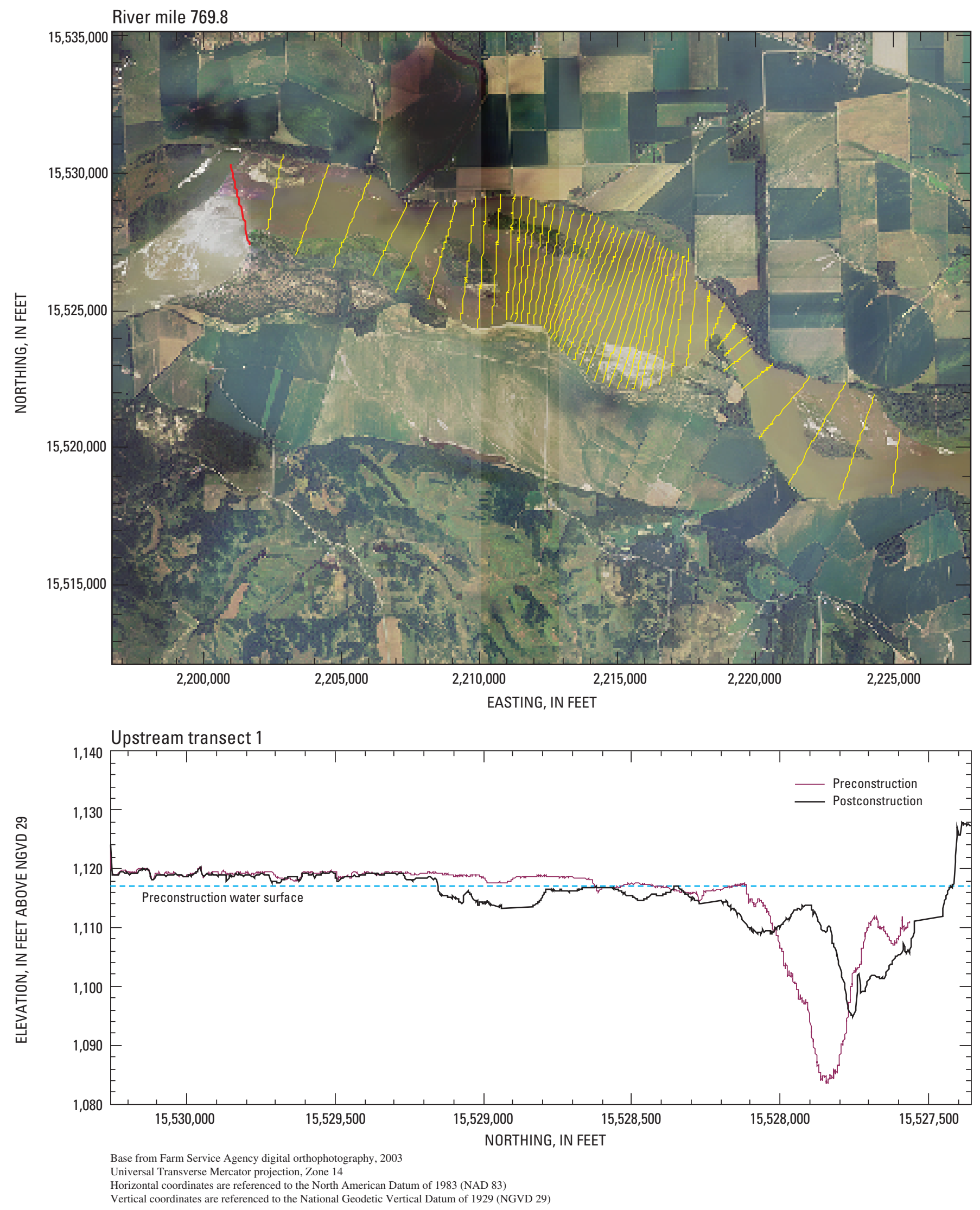

Figure A55. Location and cross section for upstream transect 1. 
River mile 769.8
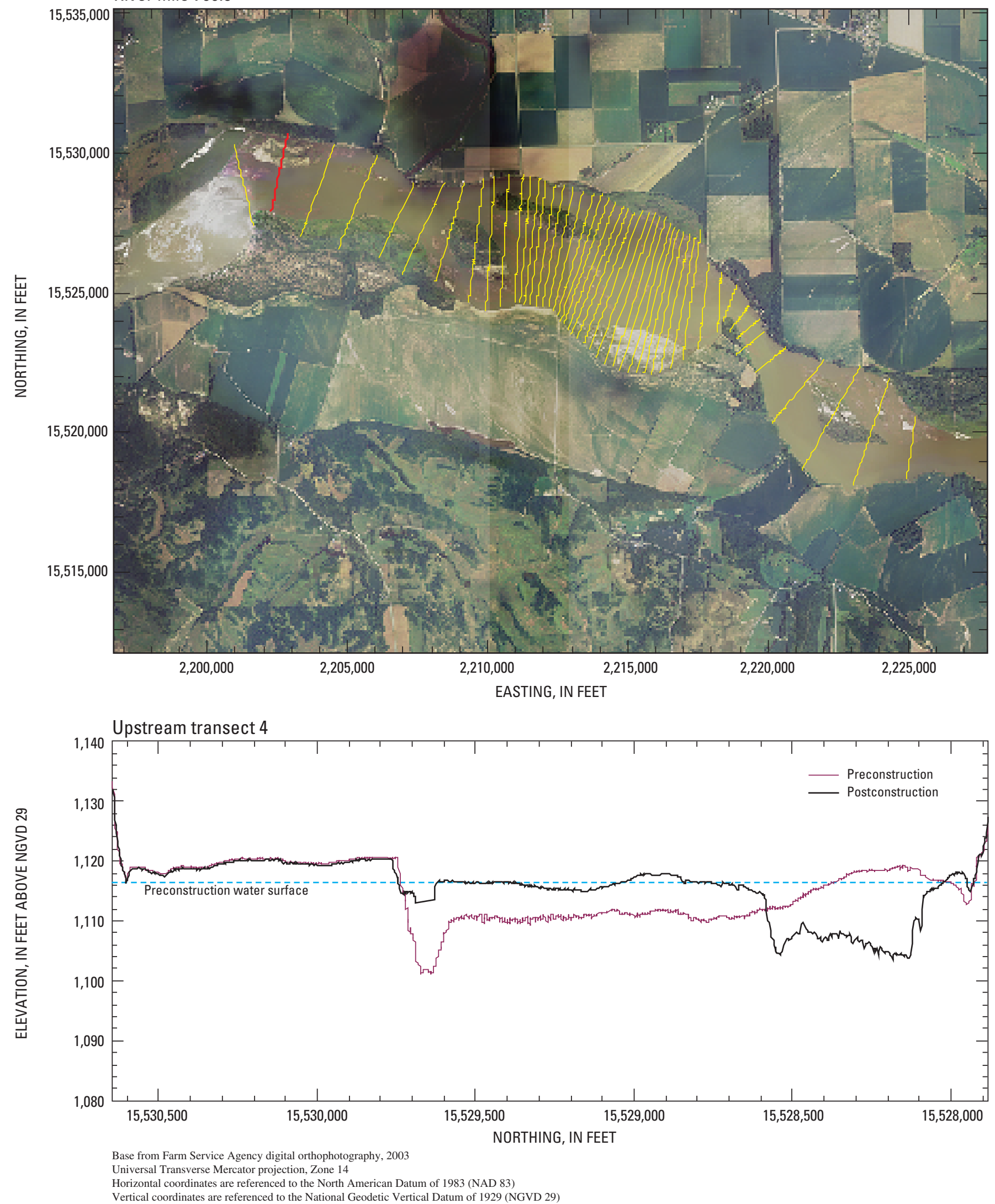

Figure A56. Location and cross section for upstream transect 4. 

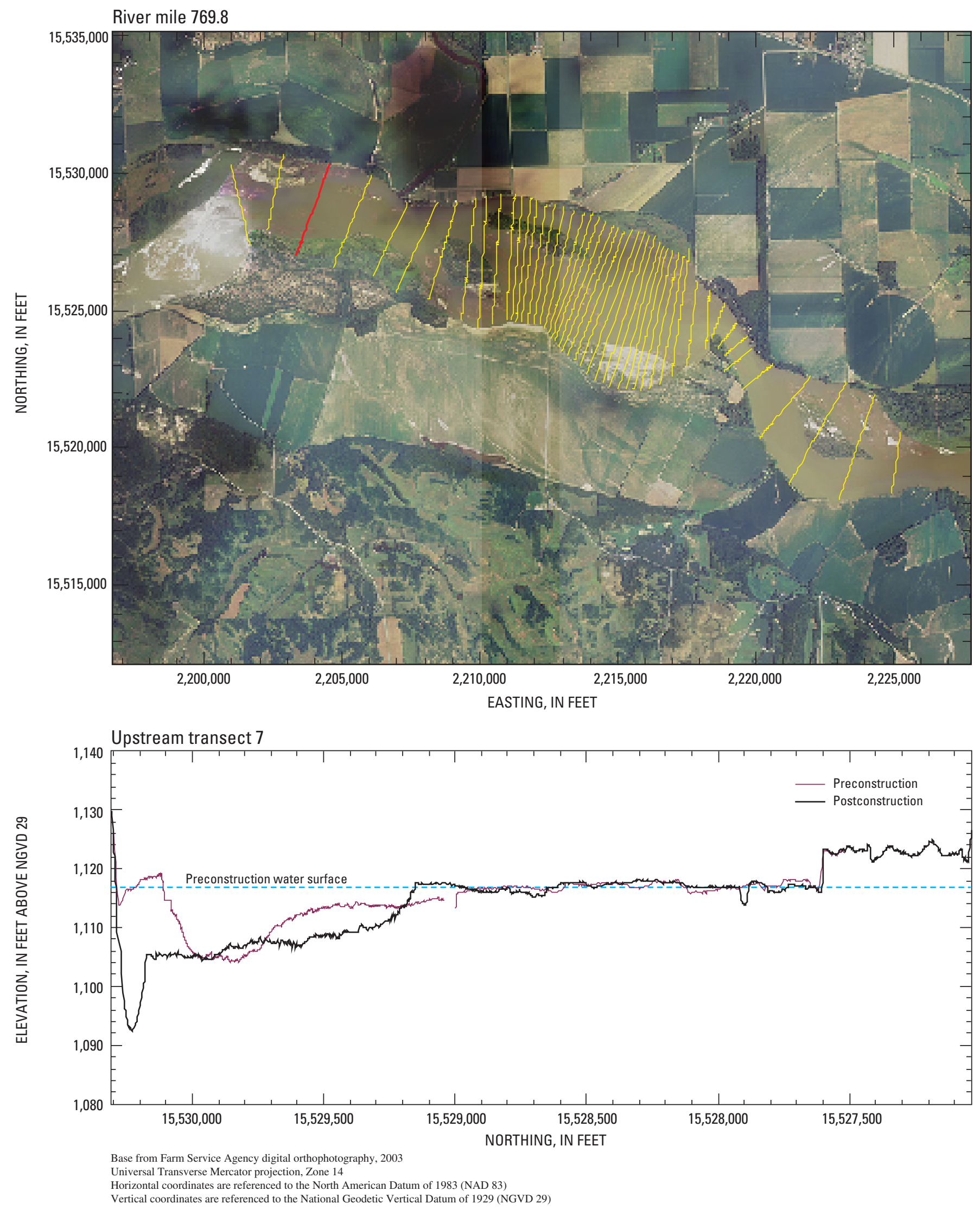

Figure A57. Location and cross section for upstream transect 7. 
River mile 769.8
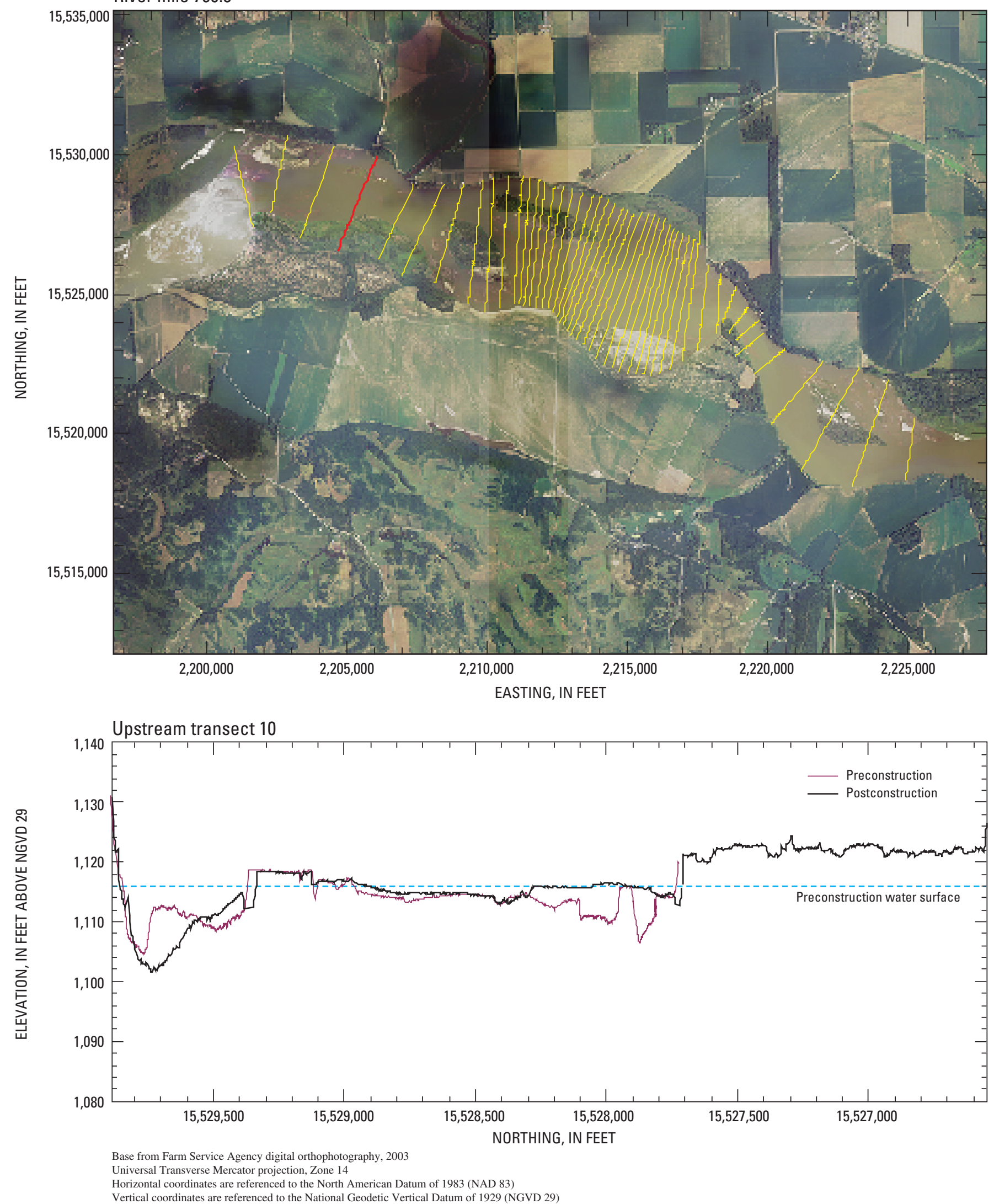

Figure A58. Location and cross section for upstream transect 10. 

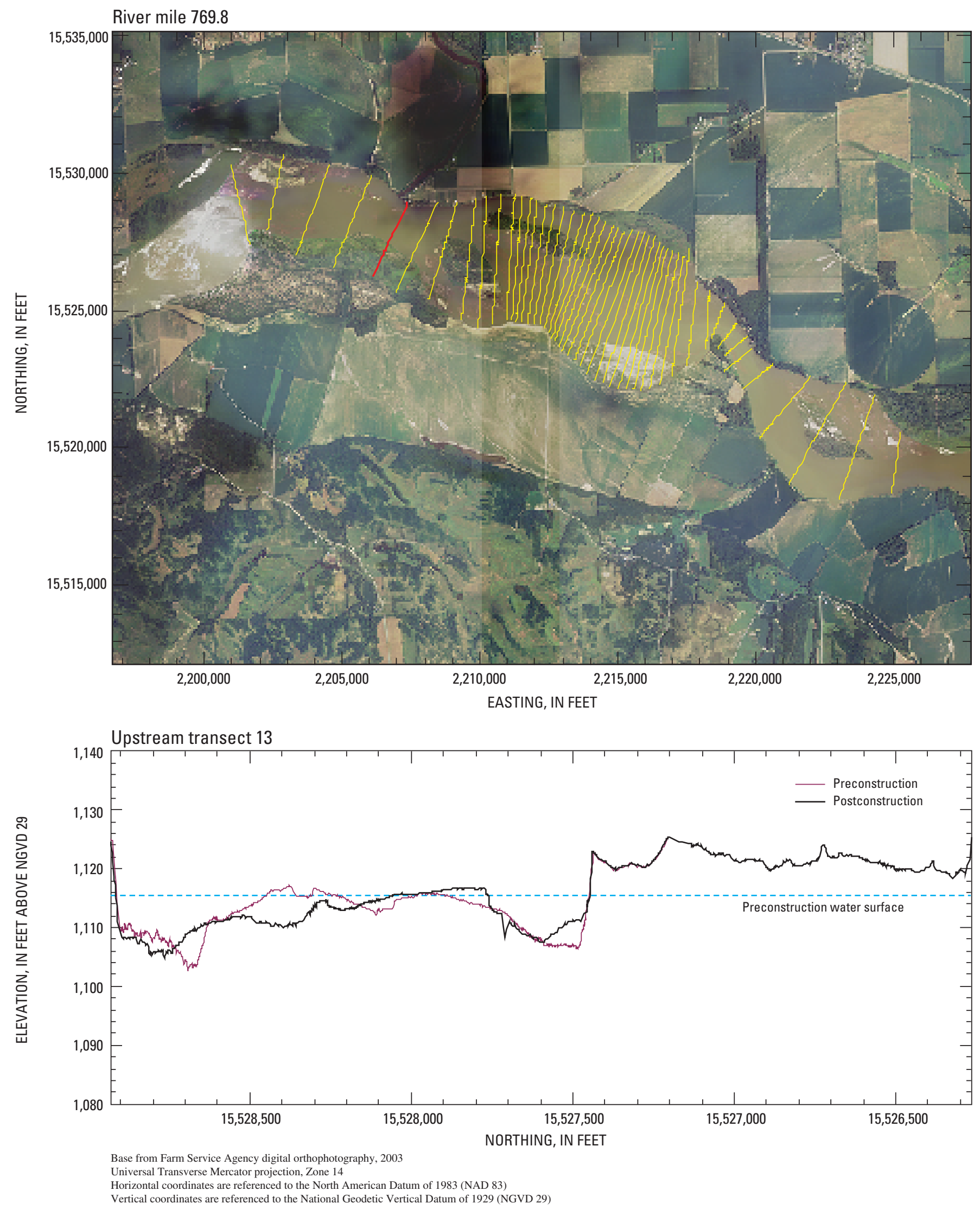

Figure A59. Location and cross section for upstream transect 13. 
River mile 769.8
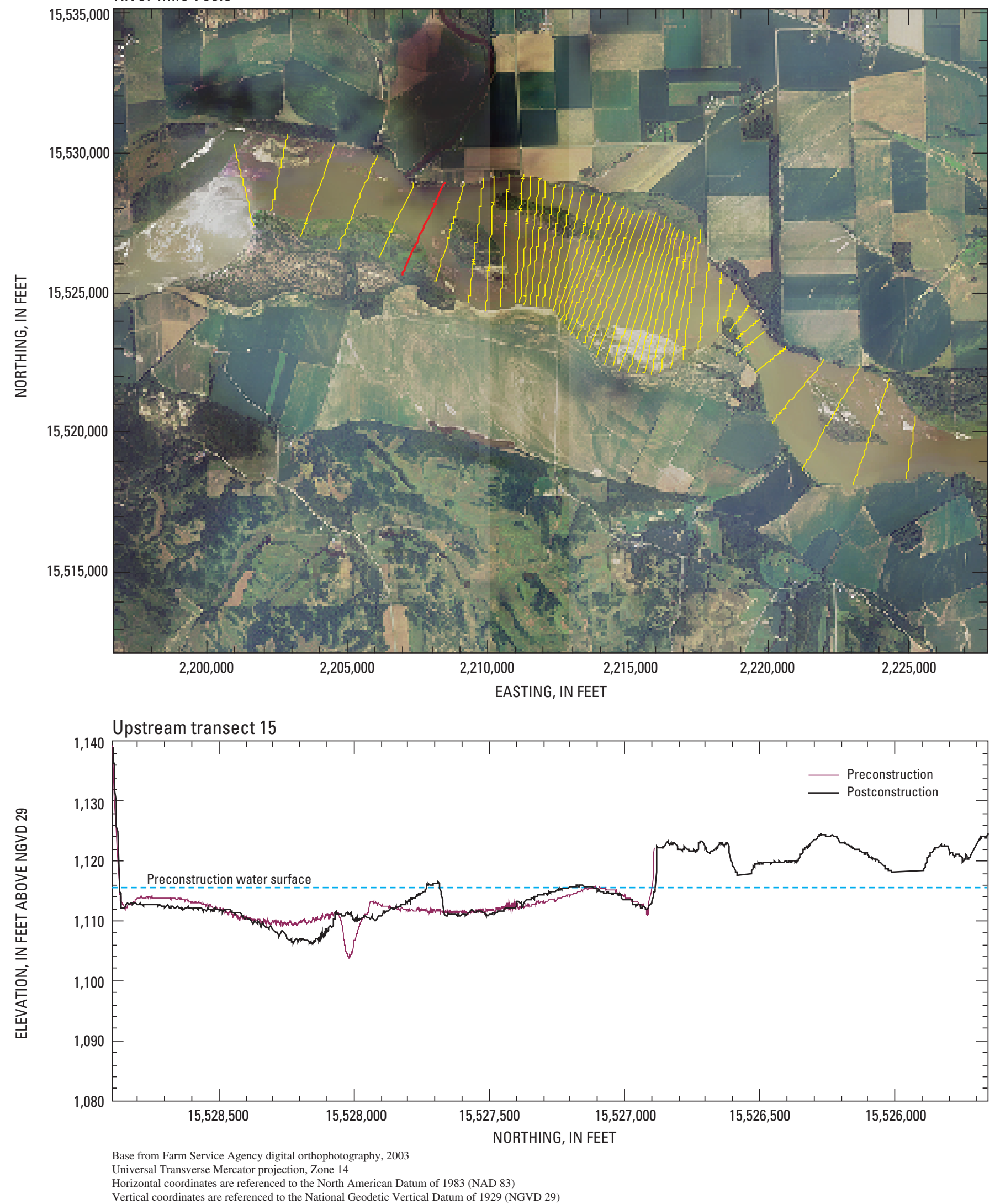

Figure A60. Location and cross section for upstream transect 15. 

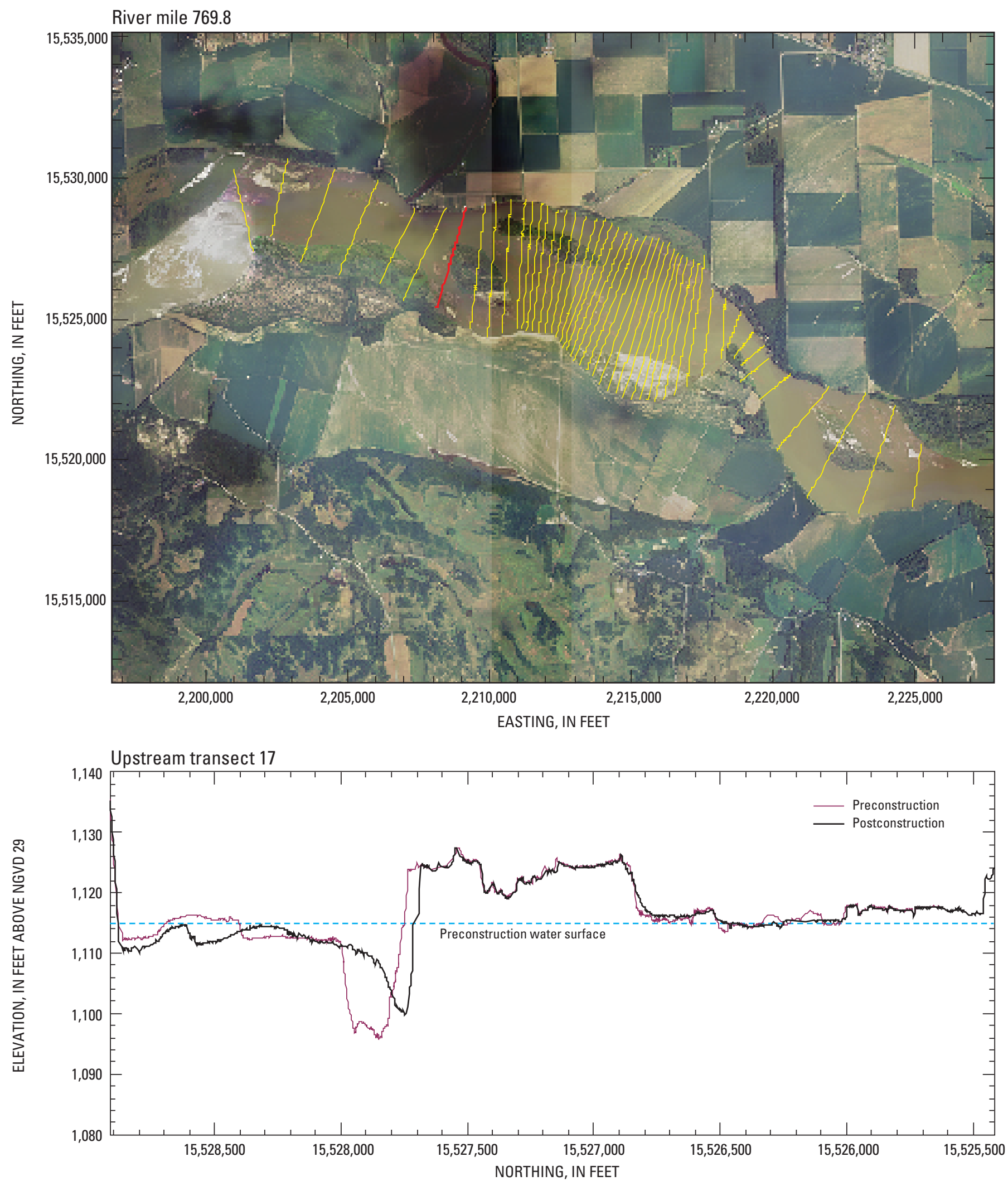

Base from Farm Service Agency digital orthophotography, 2003

Universal Transverse Mercator projection, Zone 14

Horizontal coordinates are referenced to the North American Datum of 1983 (NAD 83)

Vertical coordinates are referenced to the National Geodetic Vertical Datum of 1929 (NGVD 29)

Figure A61. Location and cross section for upstream transect 17. 
River mile 769.8
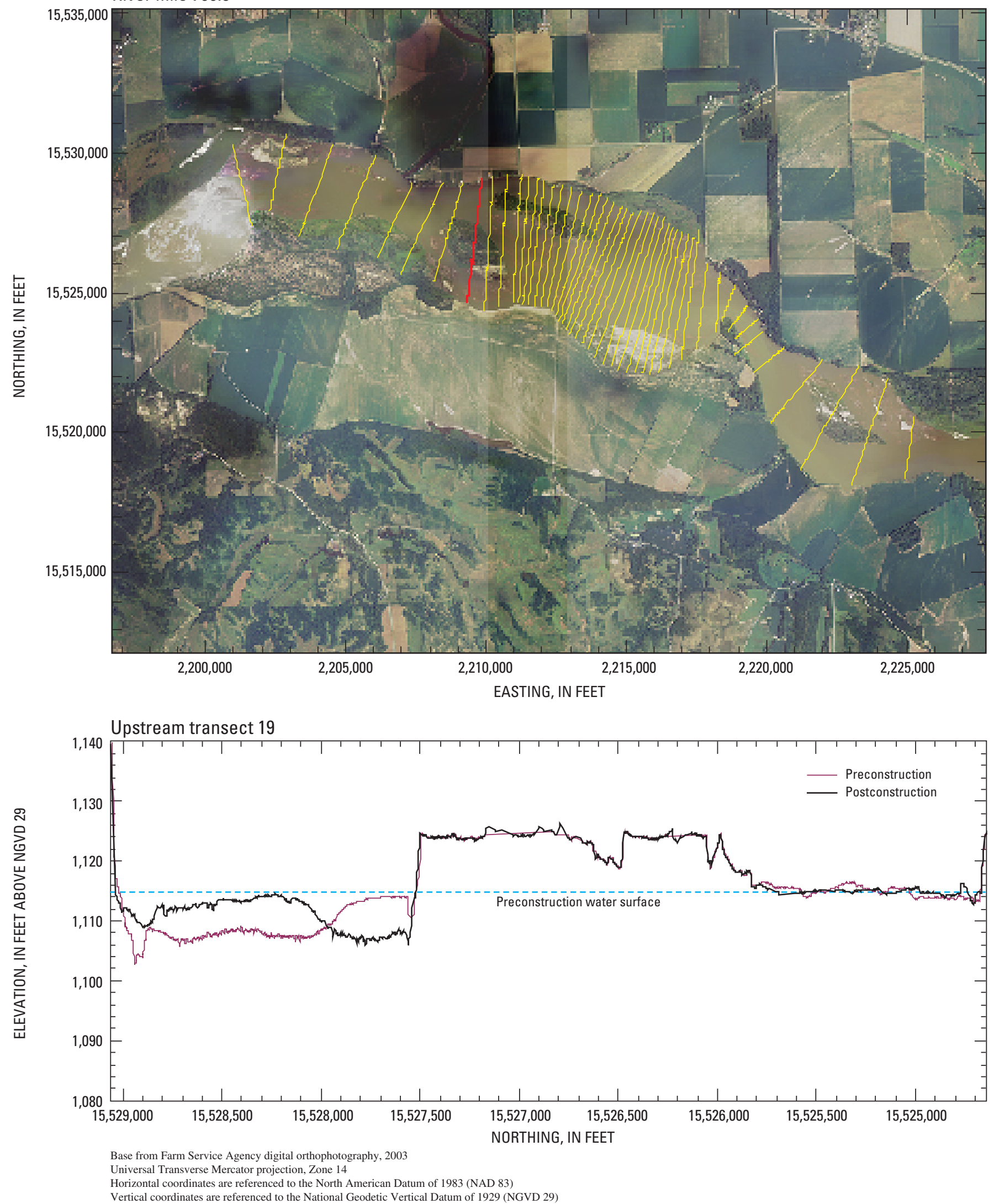

Figure A62. Location and cross section for upstream transect 19. 

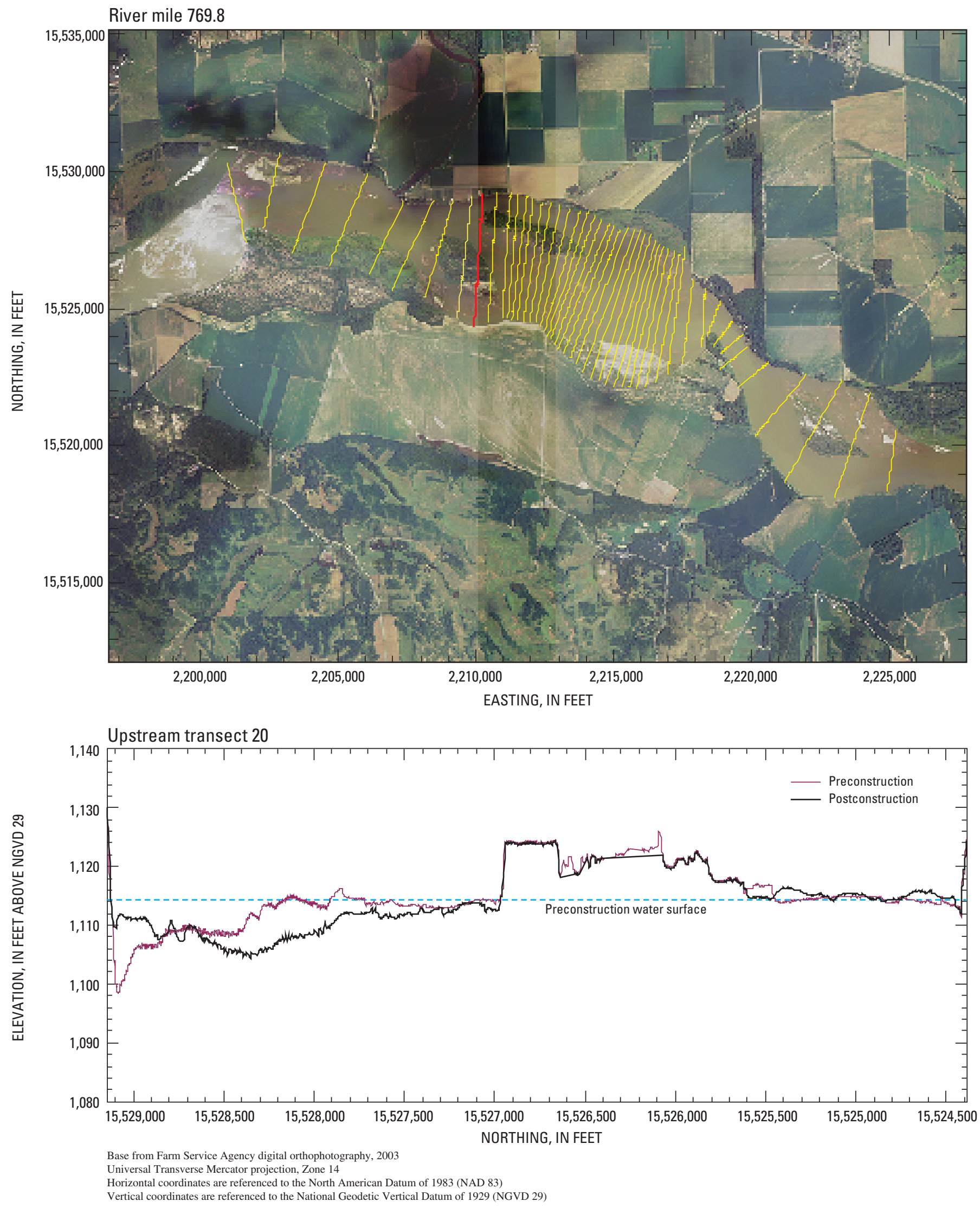

Figure A63. Location and cross section for upstream transect 20. 
River mile 769.8
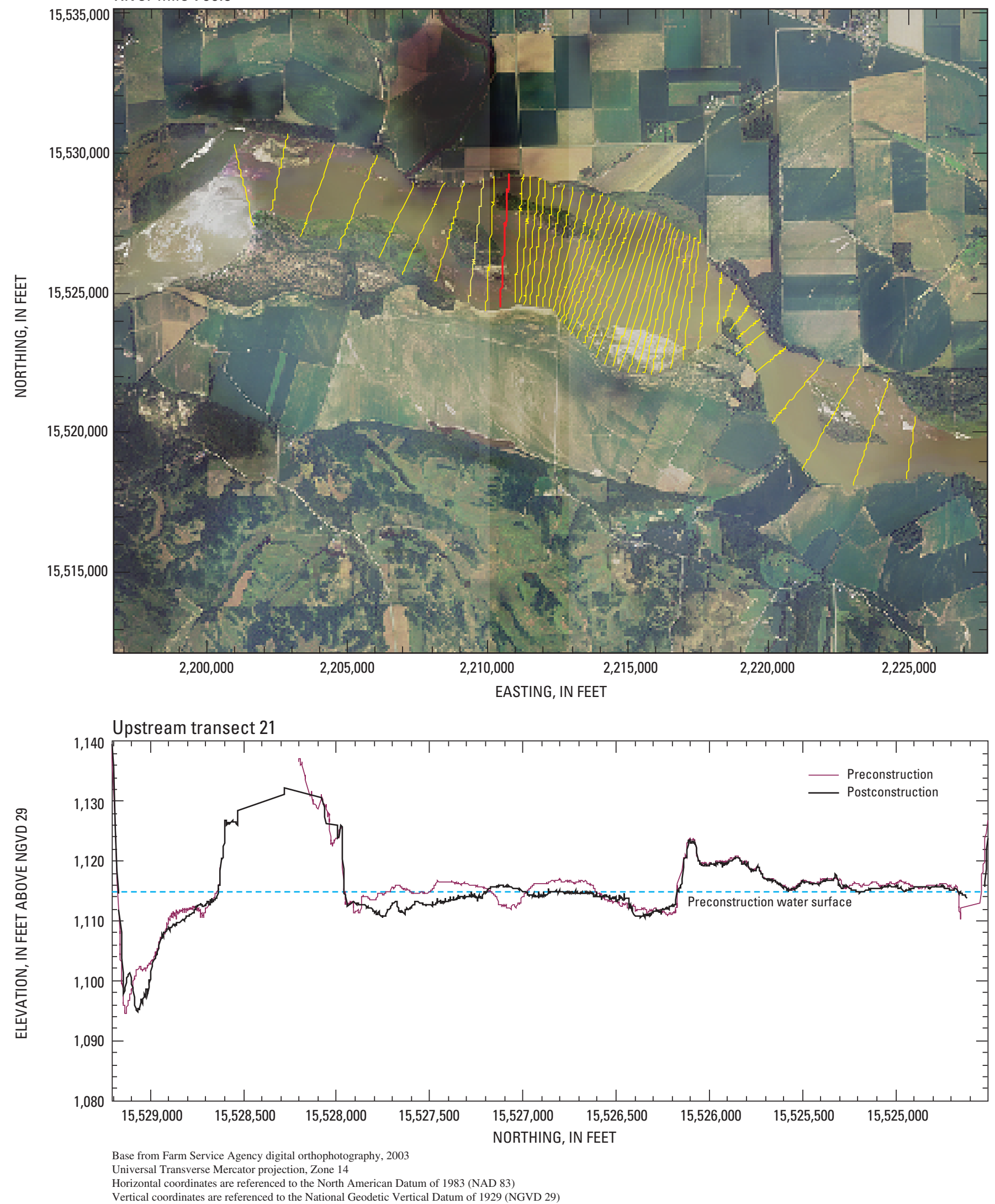

Figure A64. Location and cross section for upstream transect 21. 

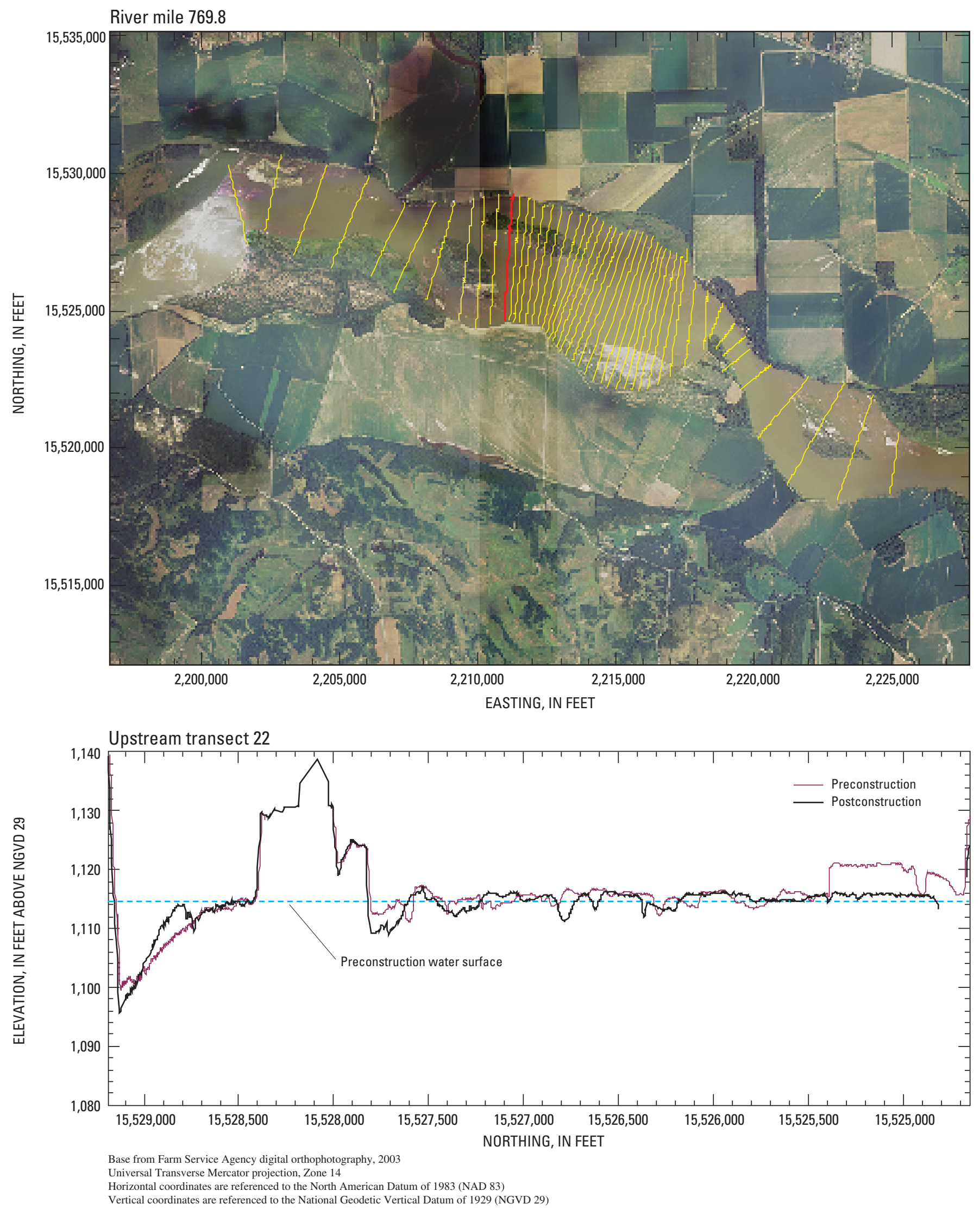

Figure A65. Location and cross section for upstream transect 22. 
River mile 769.8
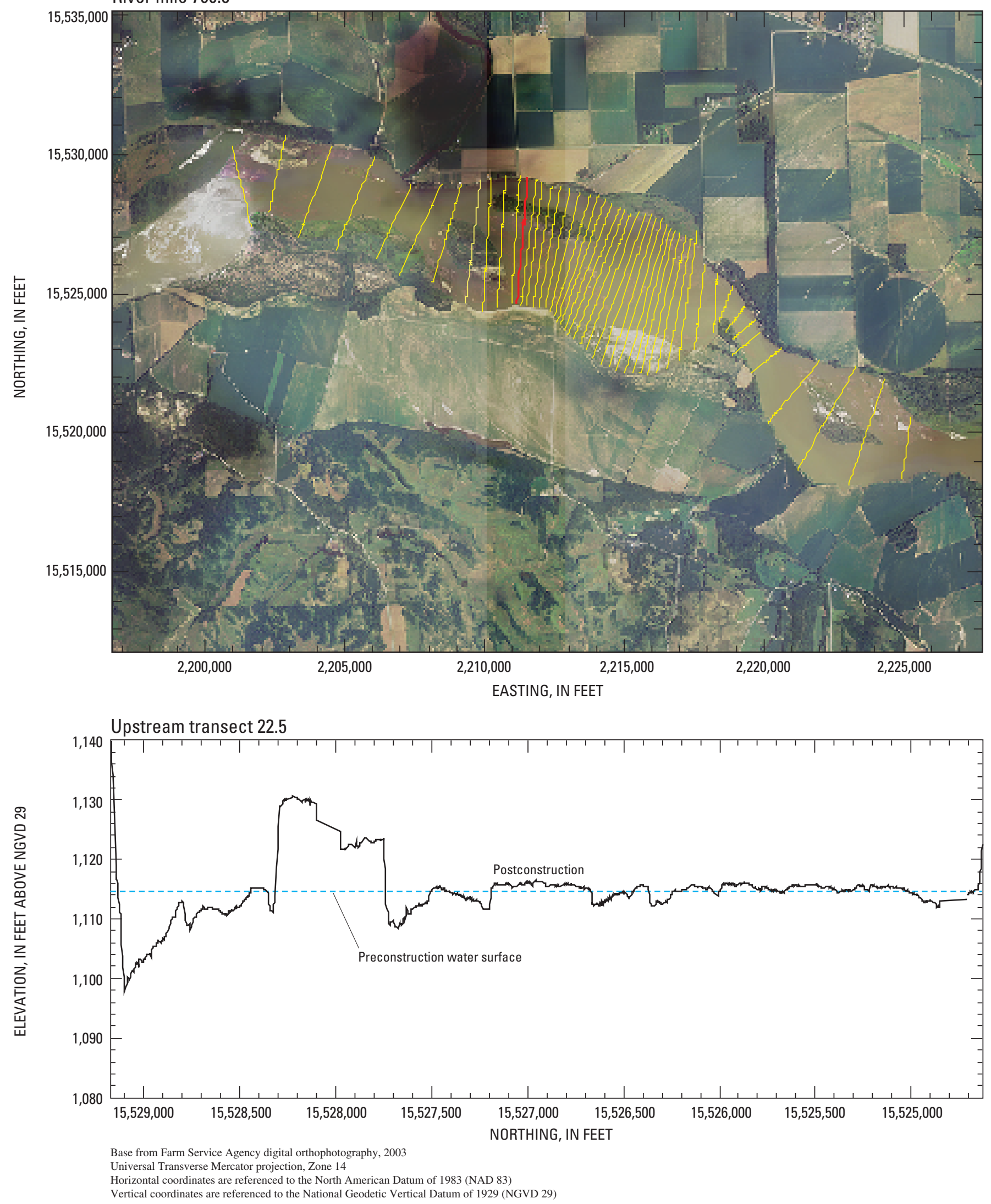

Figure A66. Location and cross section for upstream transect 22.5. 

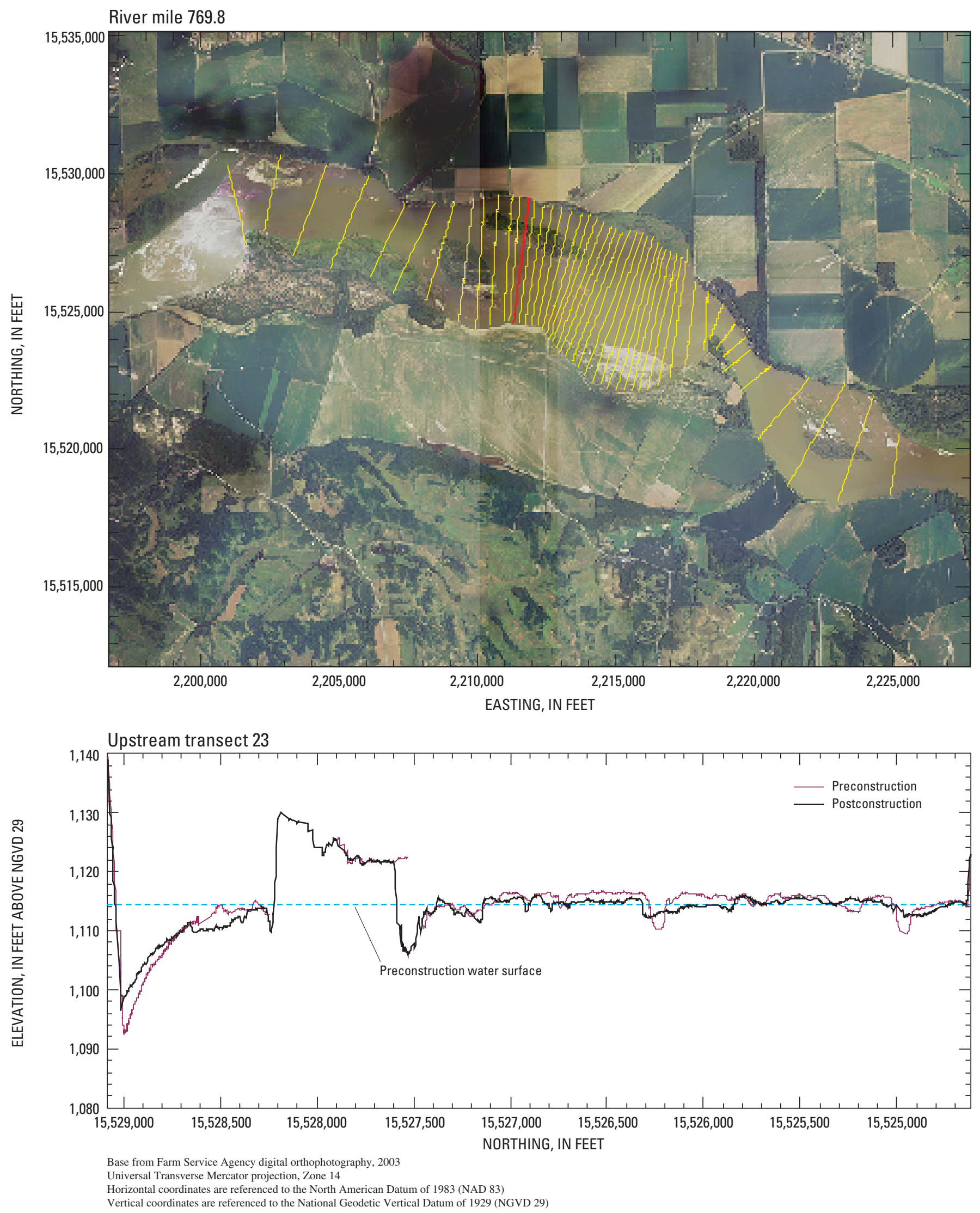

Figure A67. Location and cross section for upstream transect 23. 
River mile 769.8
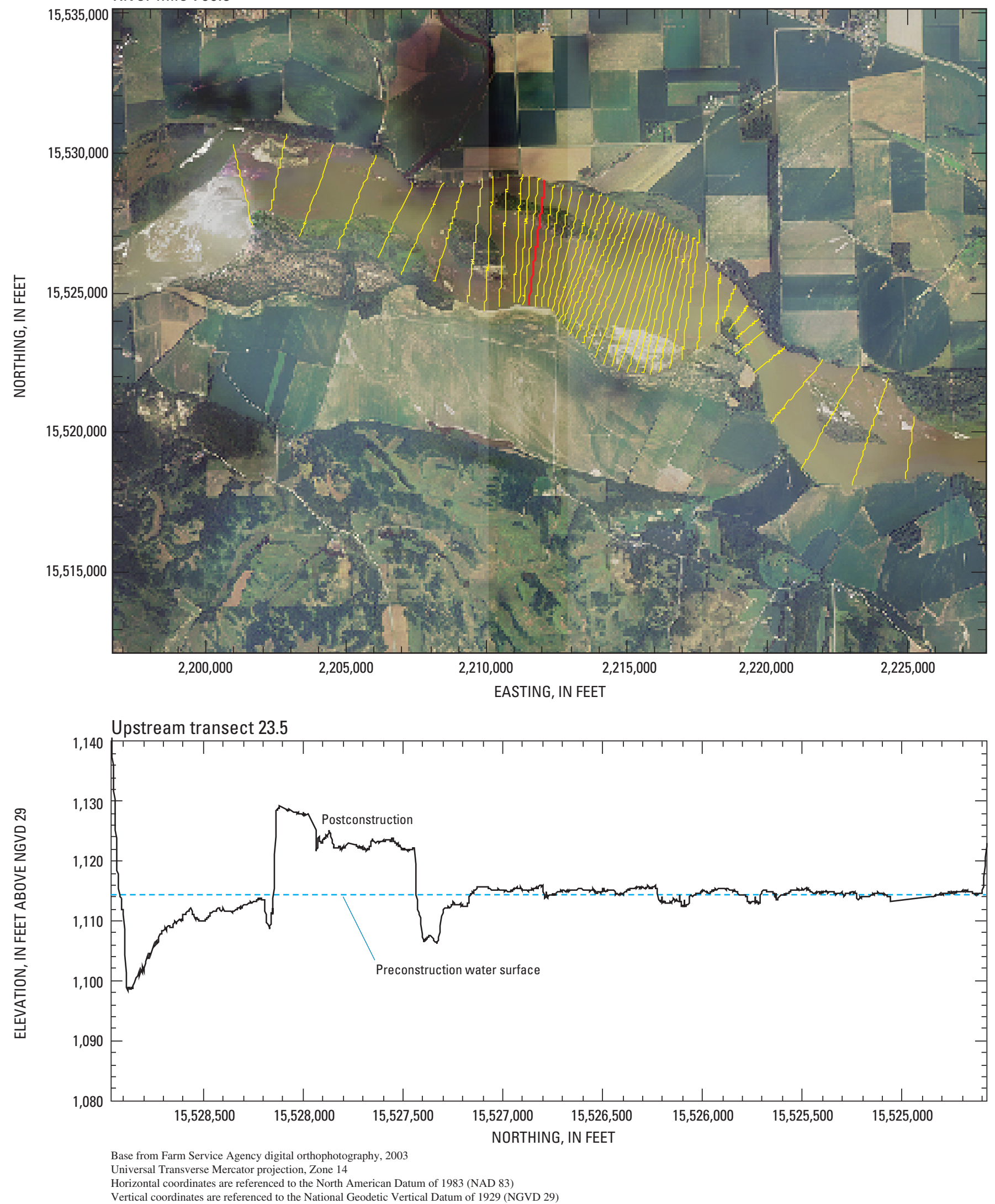

Figure A68. Location and cross section for upstream transect 23.5. 

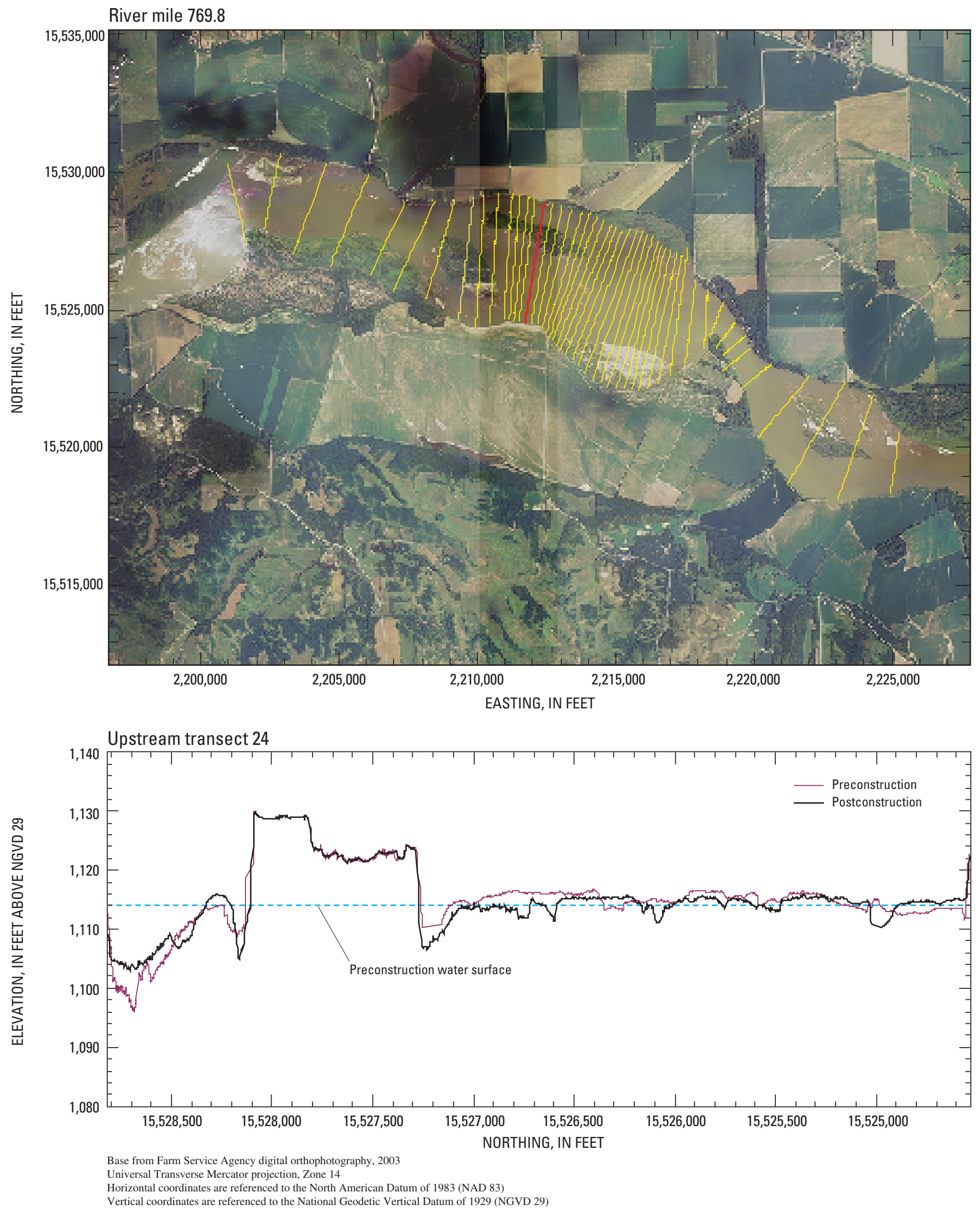

Figure A69. Location and cross section for upstream transect 24. 
River mile 769.8
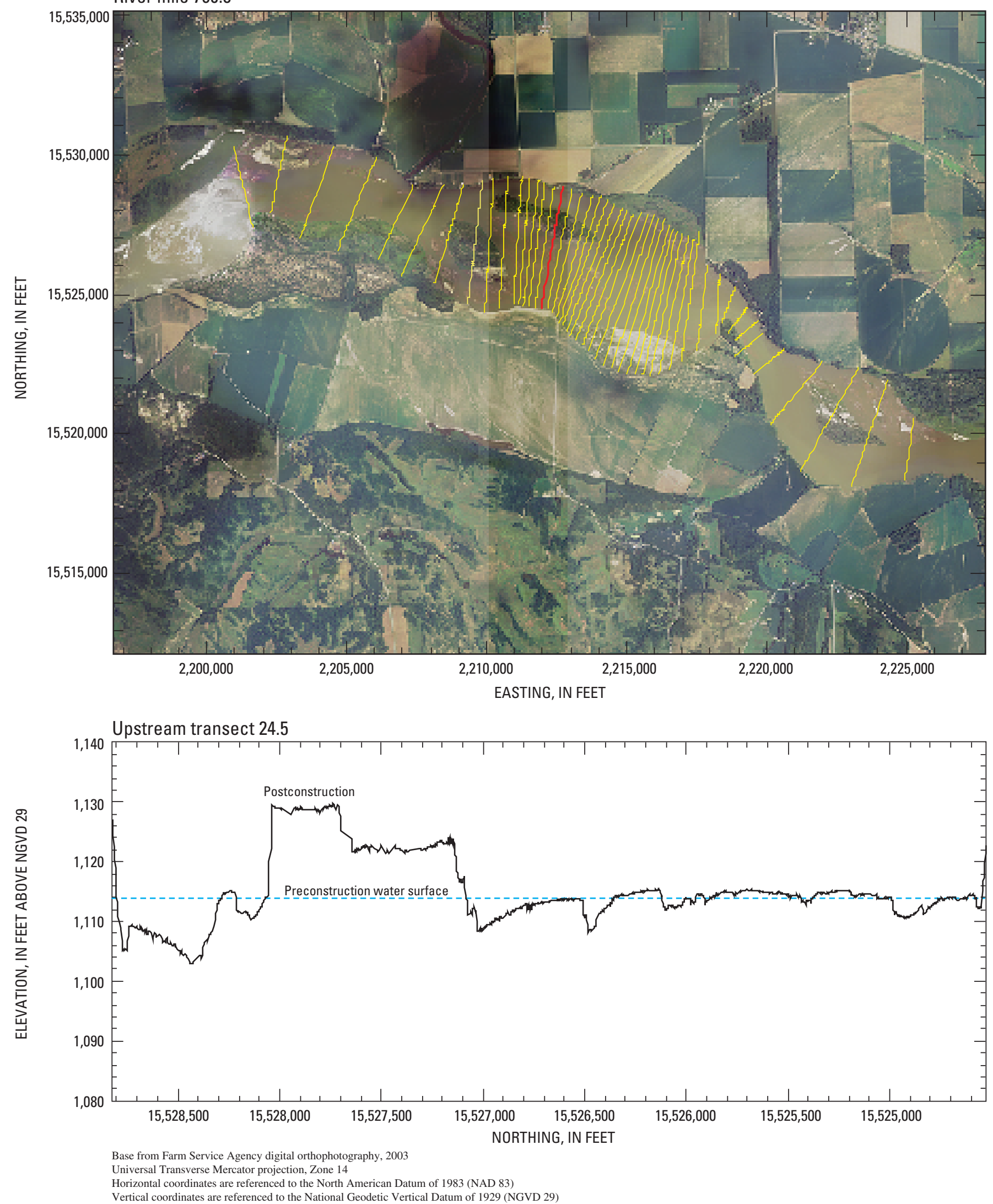

Figure A70. Location and cross section for upstream transect 24.5. 

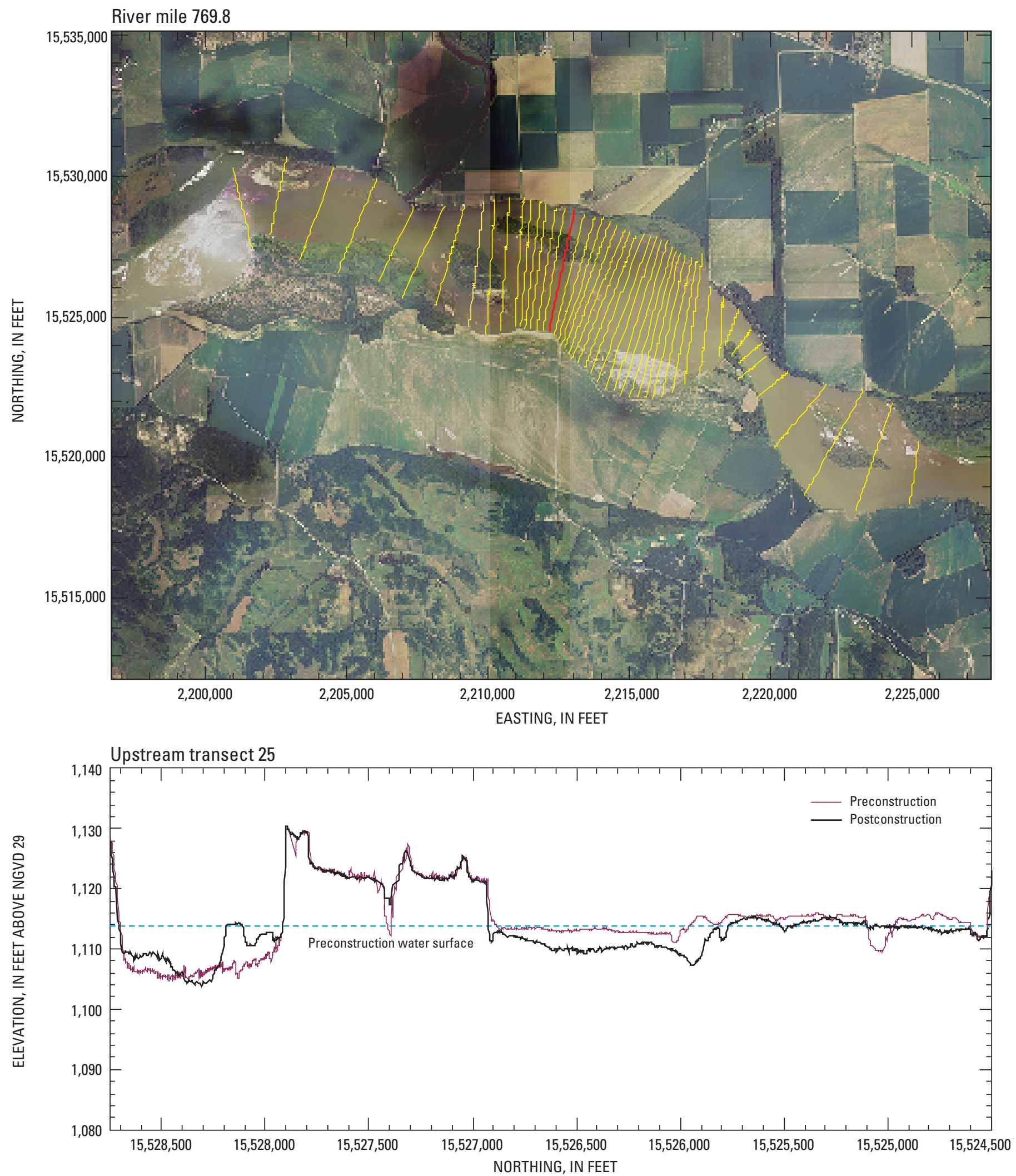

Base from Farm Service Agency digital orthophotography, 2003

Universal Transverse Mercator projection, Zone 14

Horizontal coordinates are referenced to the North American Datum of 1983 (NAD 83)

Vertical coordinates are referenced to the National Geodetic Vertical Datum of 1929 (NGVD 29)

Figure A71. Location and cross section for upstream transect 25. 

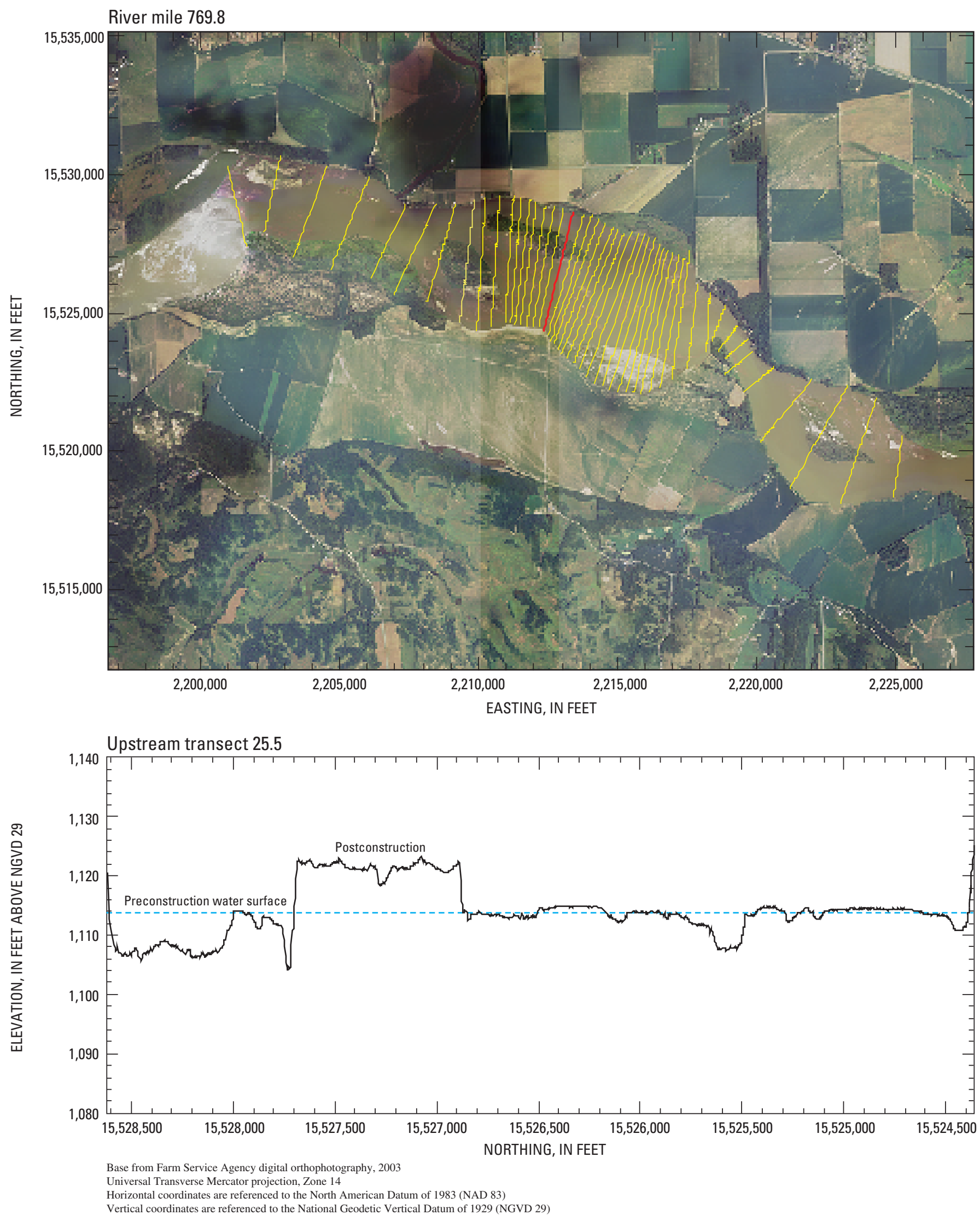

Figure A72. Location and cross section for upstream transect 25.5. 

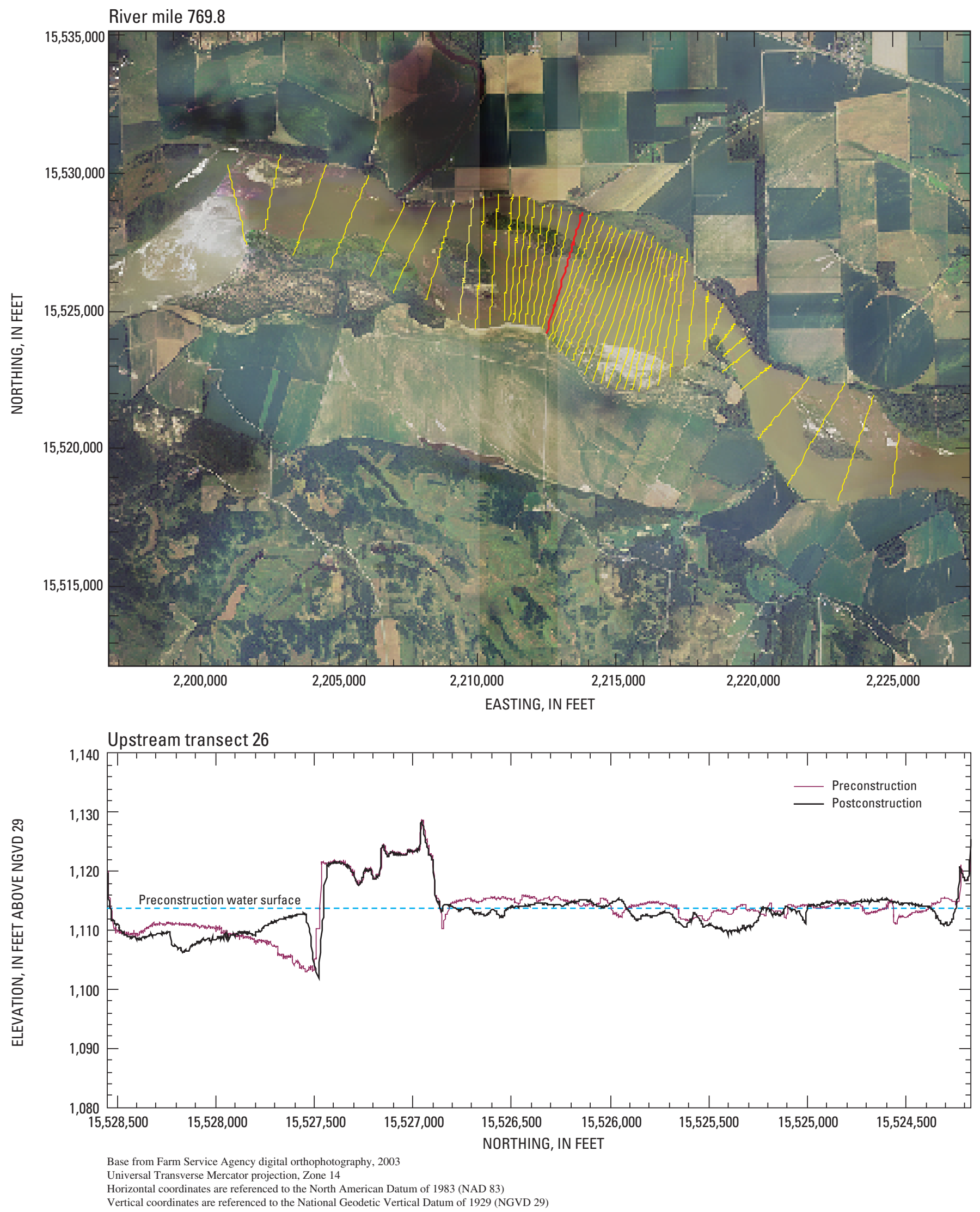

Figure A73. Location and cross section for upstream transect 26. 

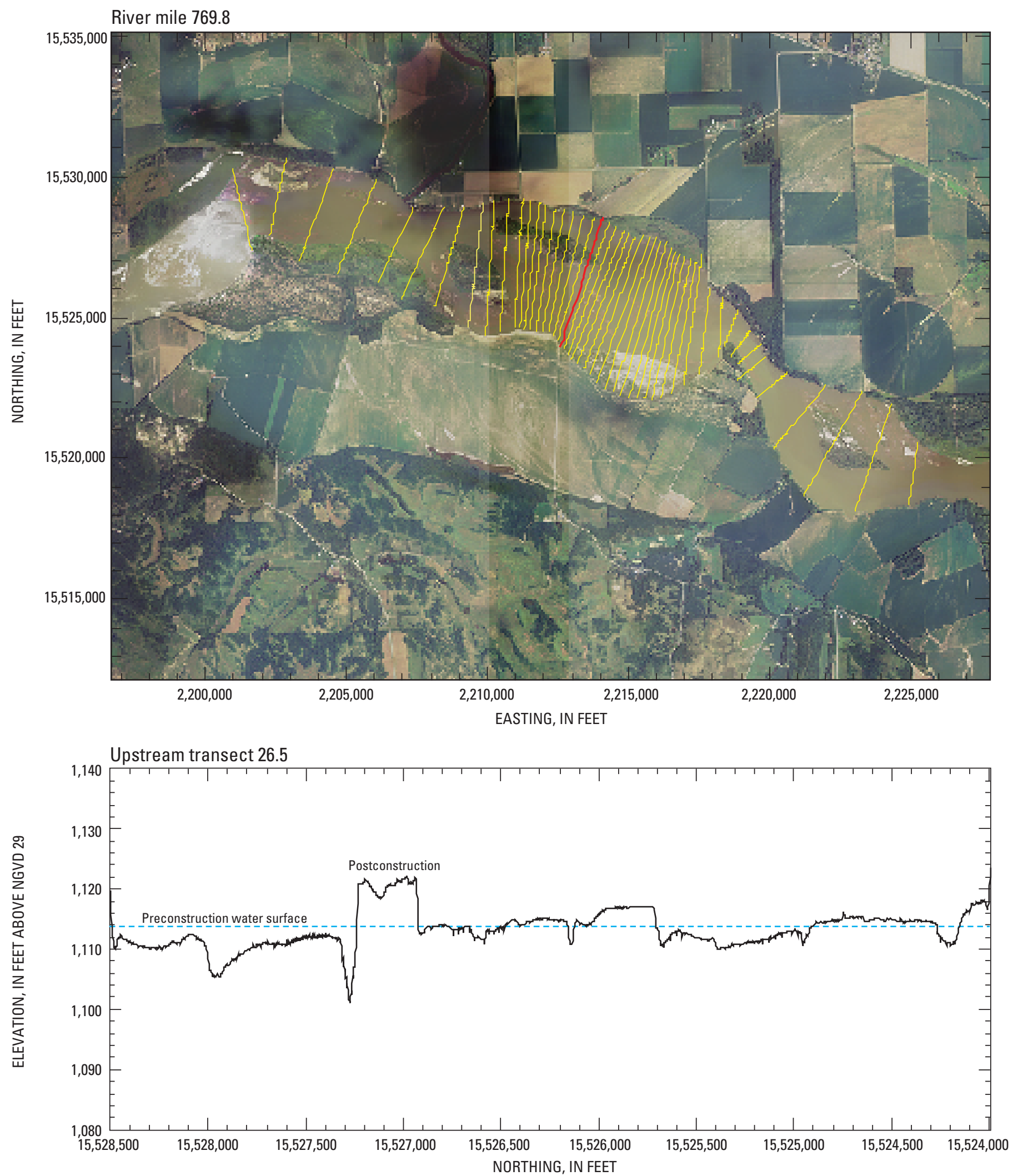

Base from Farm Service Agency digital orthophotography, 2003

Universal Transverse Mercator projection, Zone 14

Horizontal coordinates are referenced to the North American Datum of 1983 (NAD 83)

Vertical coordinates are referenced to the National Geodetic Vertical Datum of 1929 (NGVD 29)

Figure A74. Location and cross section for upstream transect 26.5. 

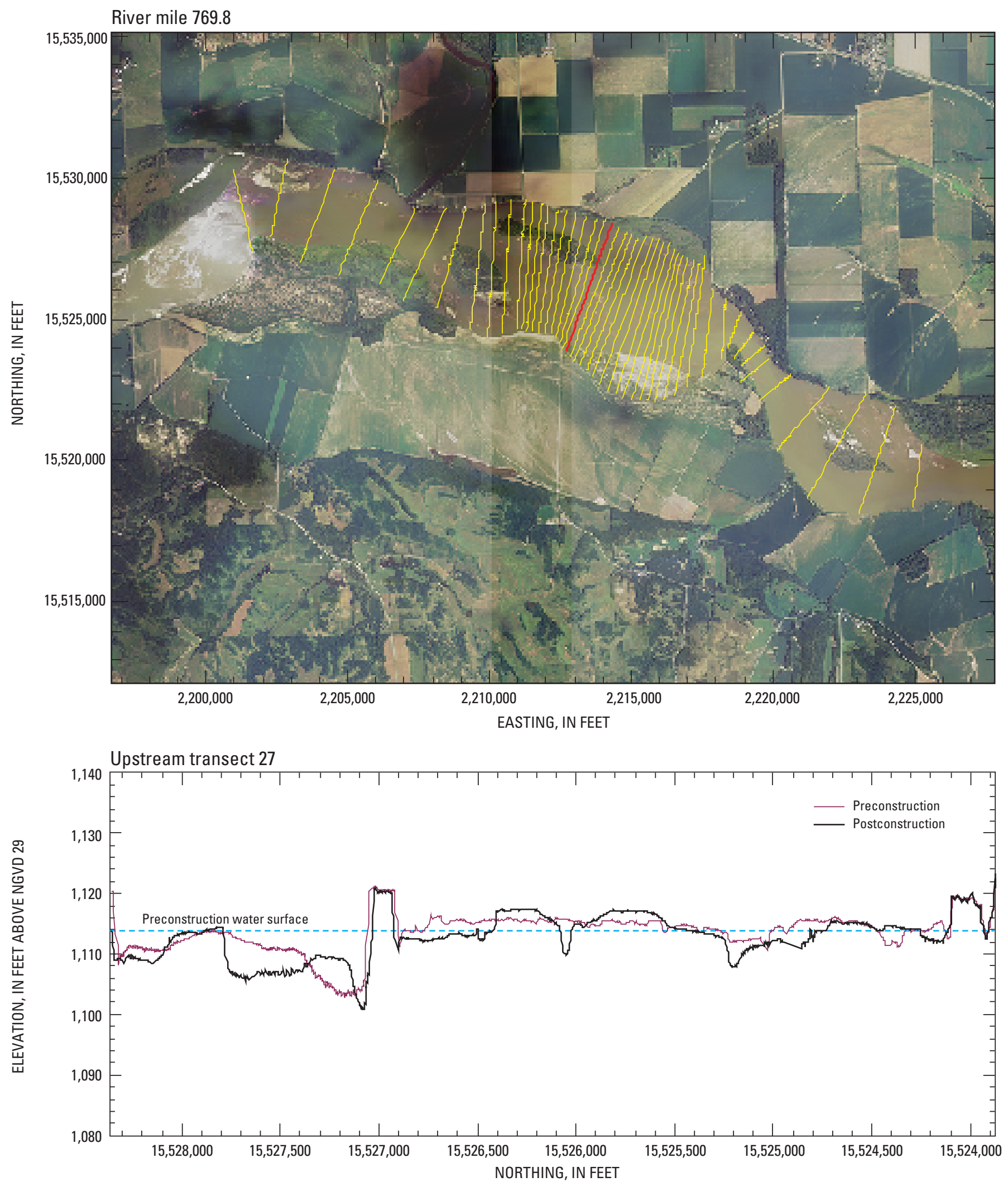

Base from Farm Service Agency digital orthophotography, 2003

Universal Transverse Mercator projection, Zone 14

Horizontal coordinates are referenced to the North American Datum of 1983 (NAD 83)

Vertical coordinates are referenced to the National Geodetic Vertical Datum of 1929 (NGVD 29)

Figure A75. Location and cross section for upstream transect 27. 

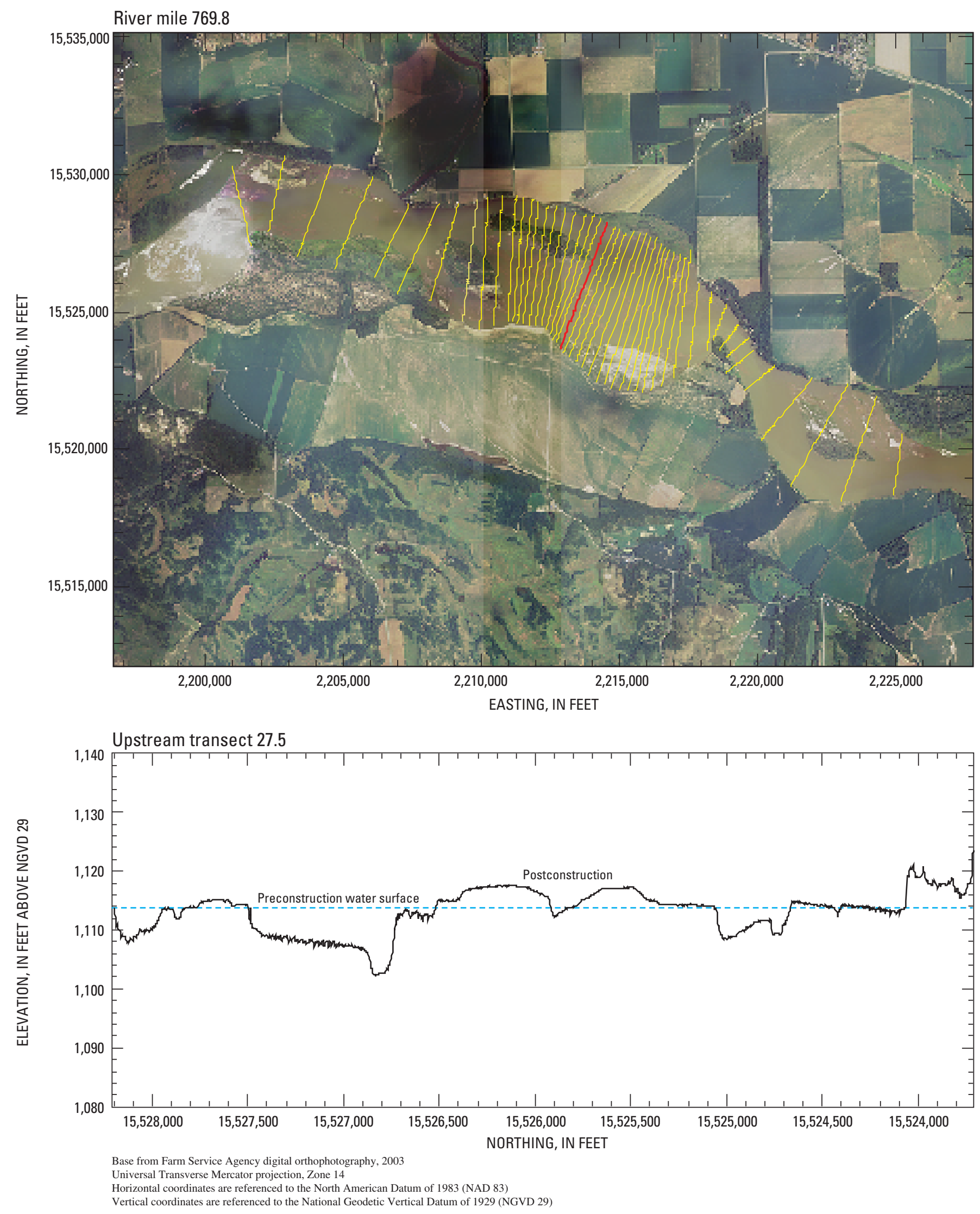

Figure A76. Location and cross section for upstream transect 27.5. 

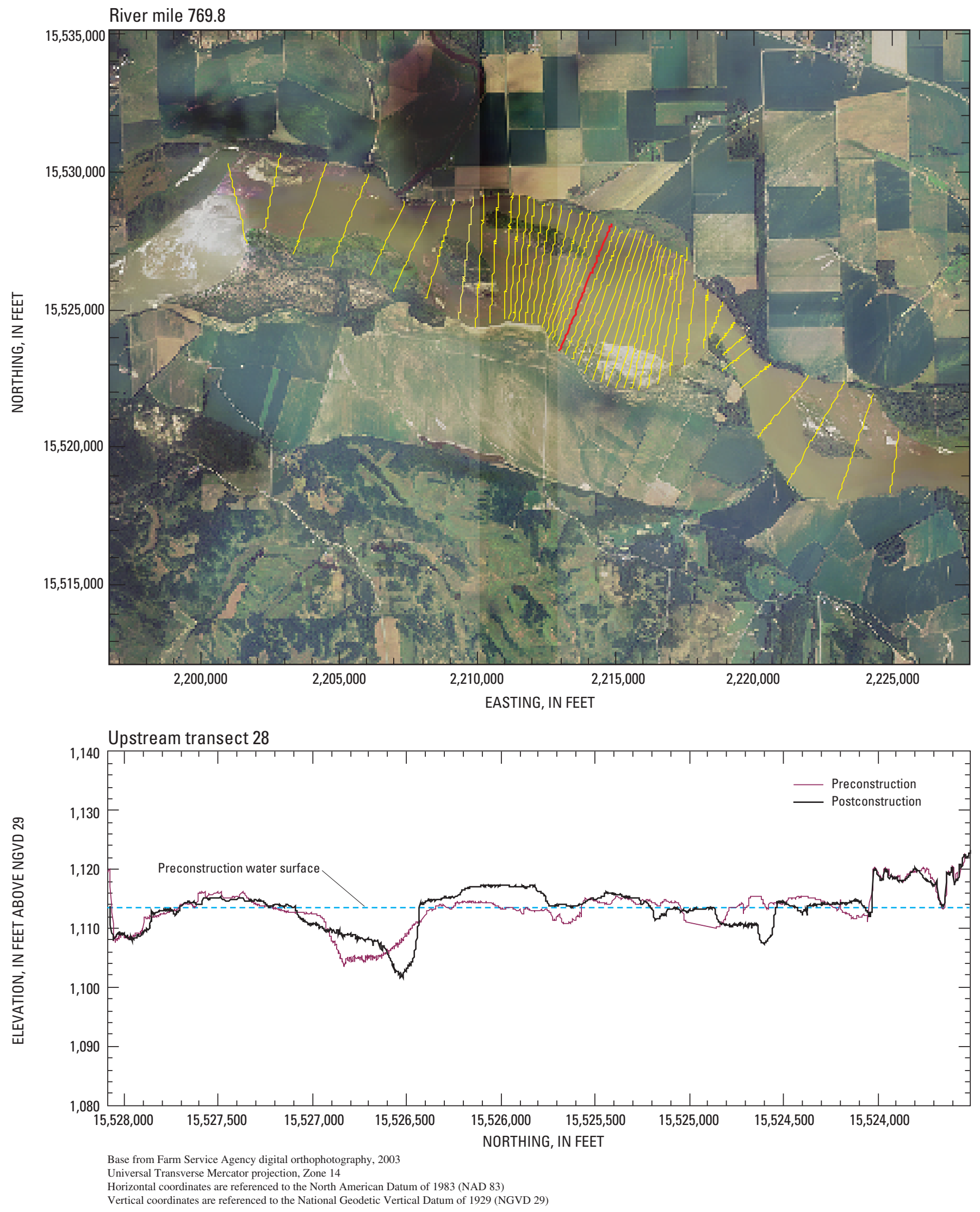

Figure A77. Location and cross section for upstream transect 28. 
River mile 769.8
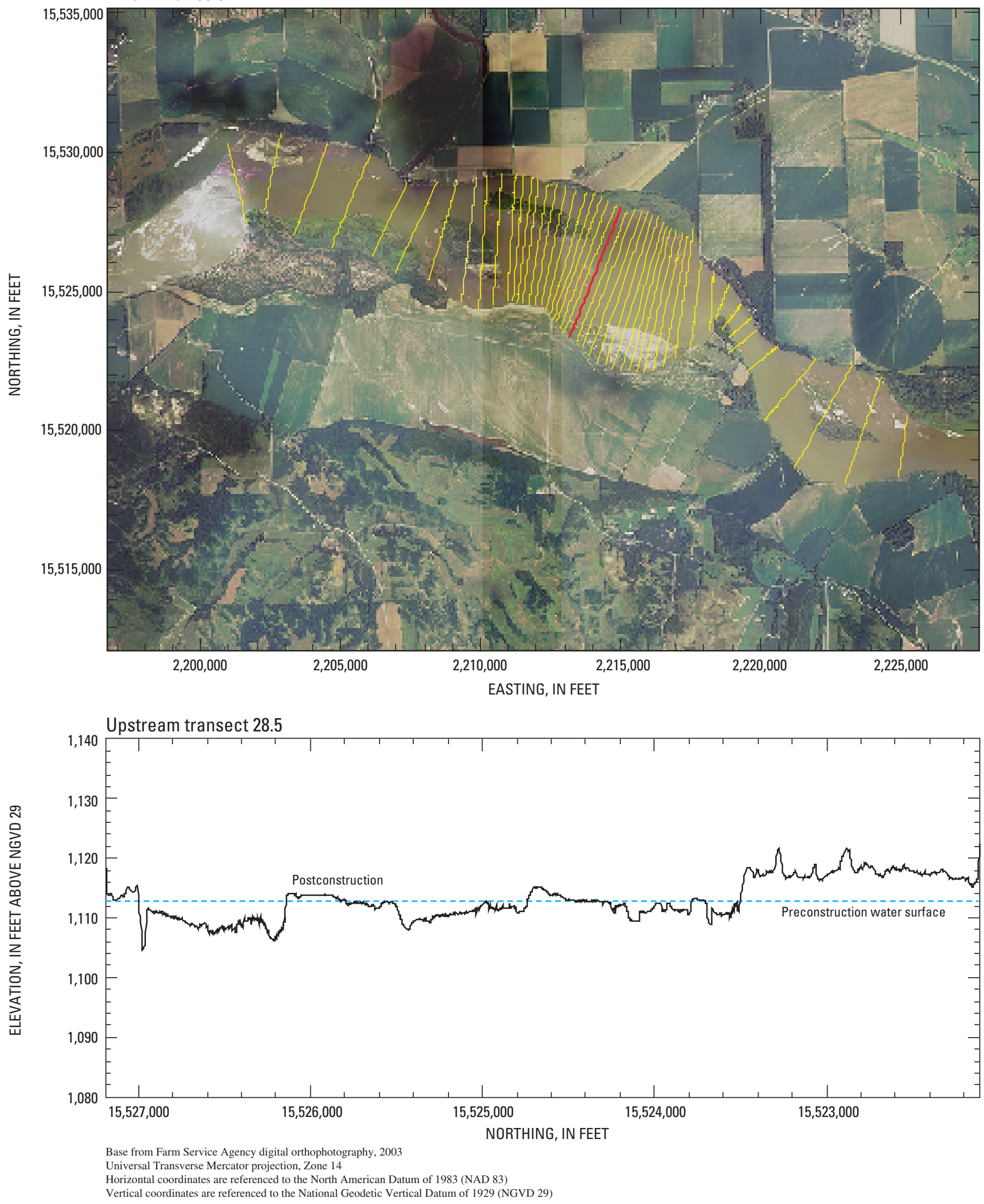

Figure A78. Location and cross section for upstream transect 28.5. 

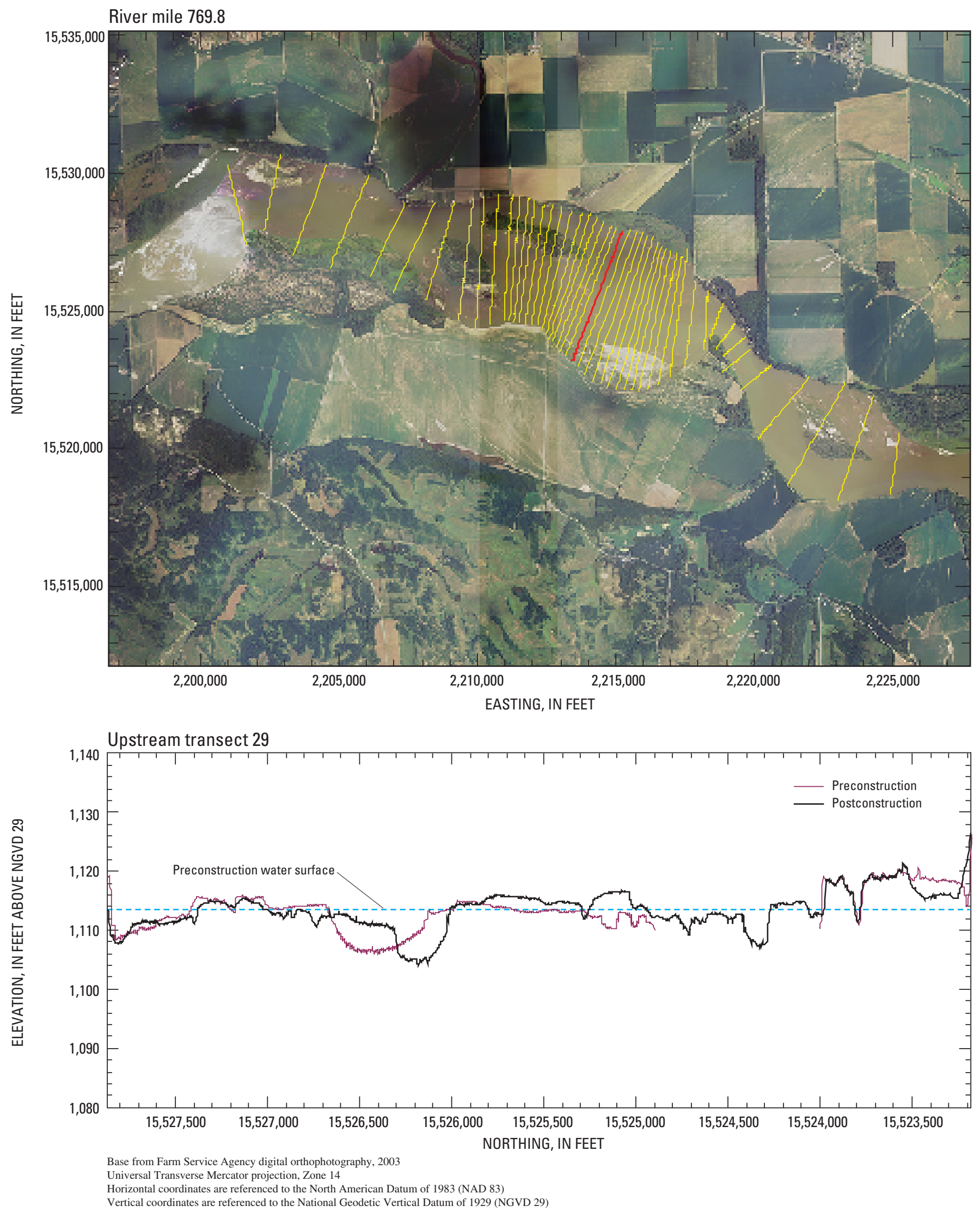

Figure A79. Location and cross section for upstream transect 29. 

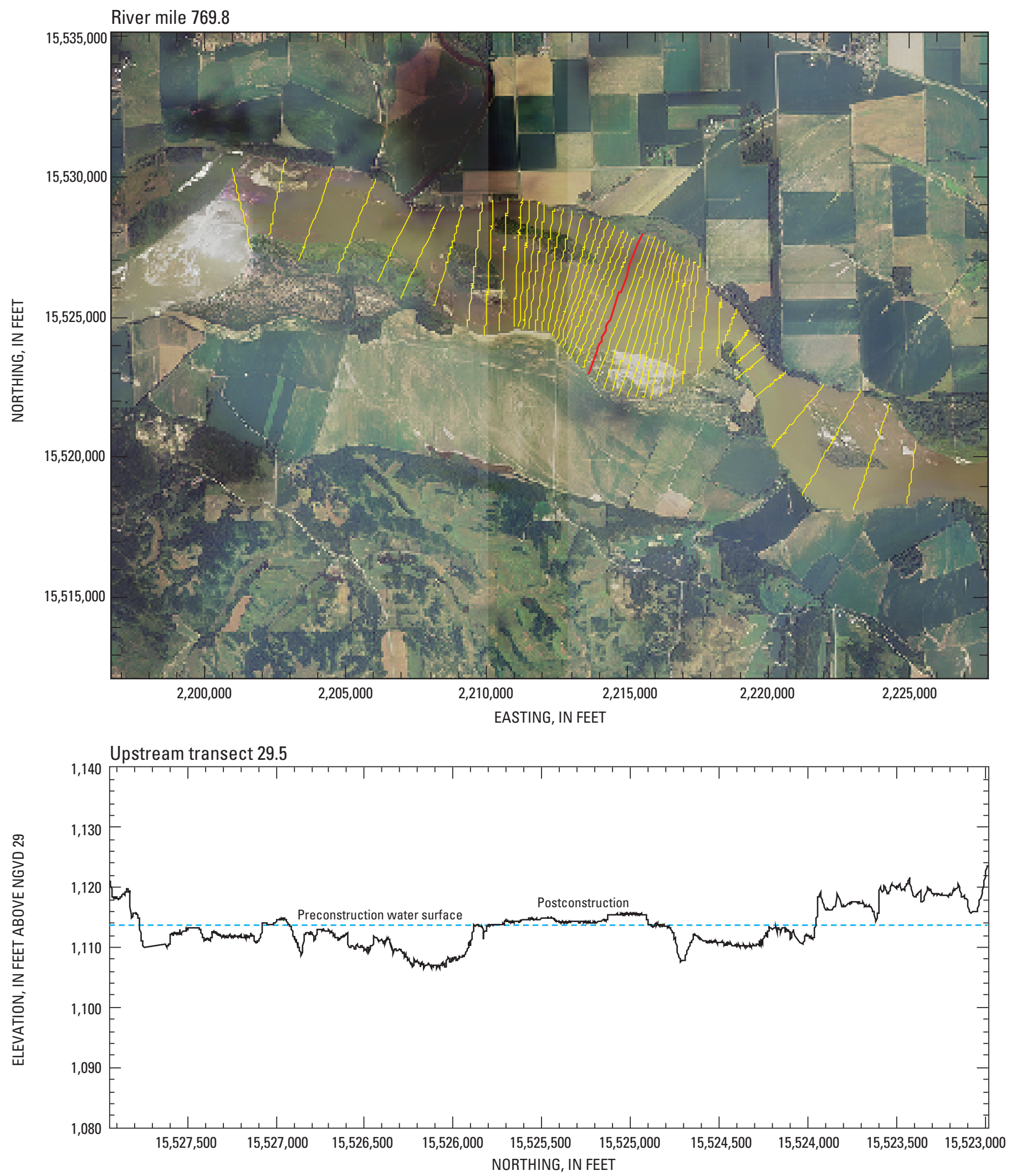

Base from Farm Service Agency digital orthophotography, 2003

Universal Transverse Mercator projection, Zone 14

Horizontal coordinates are referenced to the North American Datum of 1983 (NAD 83)

Vertical coordinates are referenced to the National Geodetic Vertical Datum of 1929 (NGVD 29)

Figure A80. Location and cross section for upstream transect 29.5. 

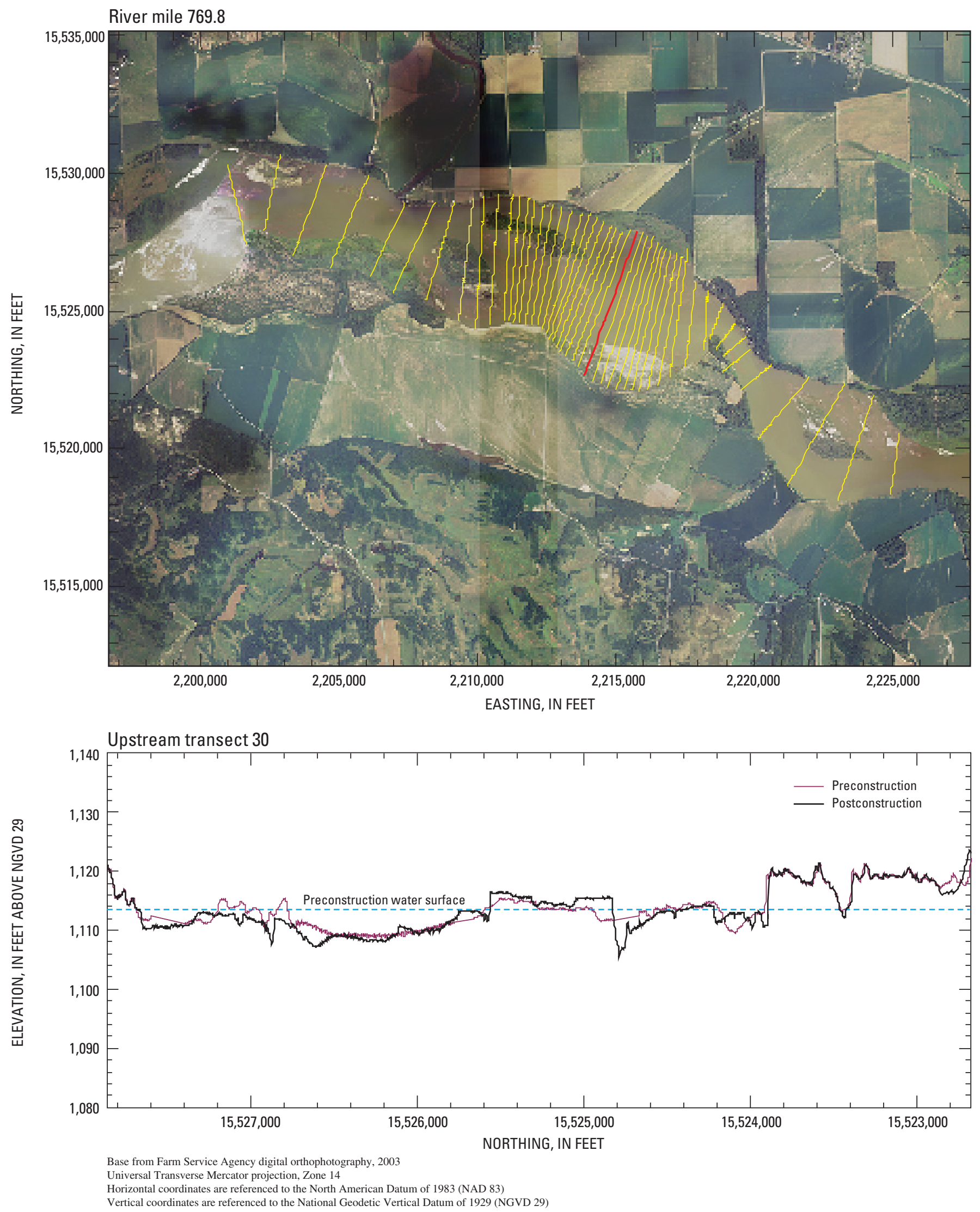

Figure A81. Location and cross section for upstream transect 30 . 

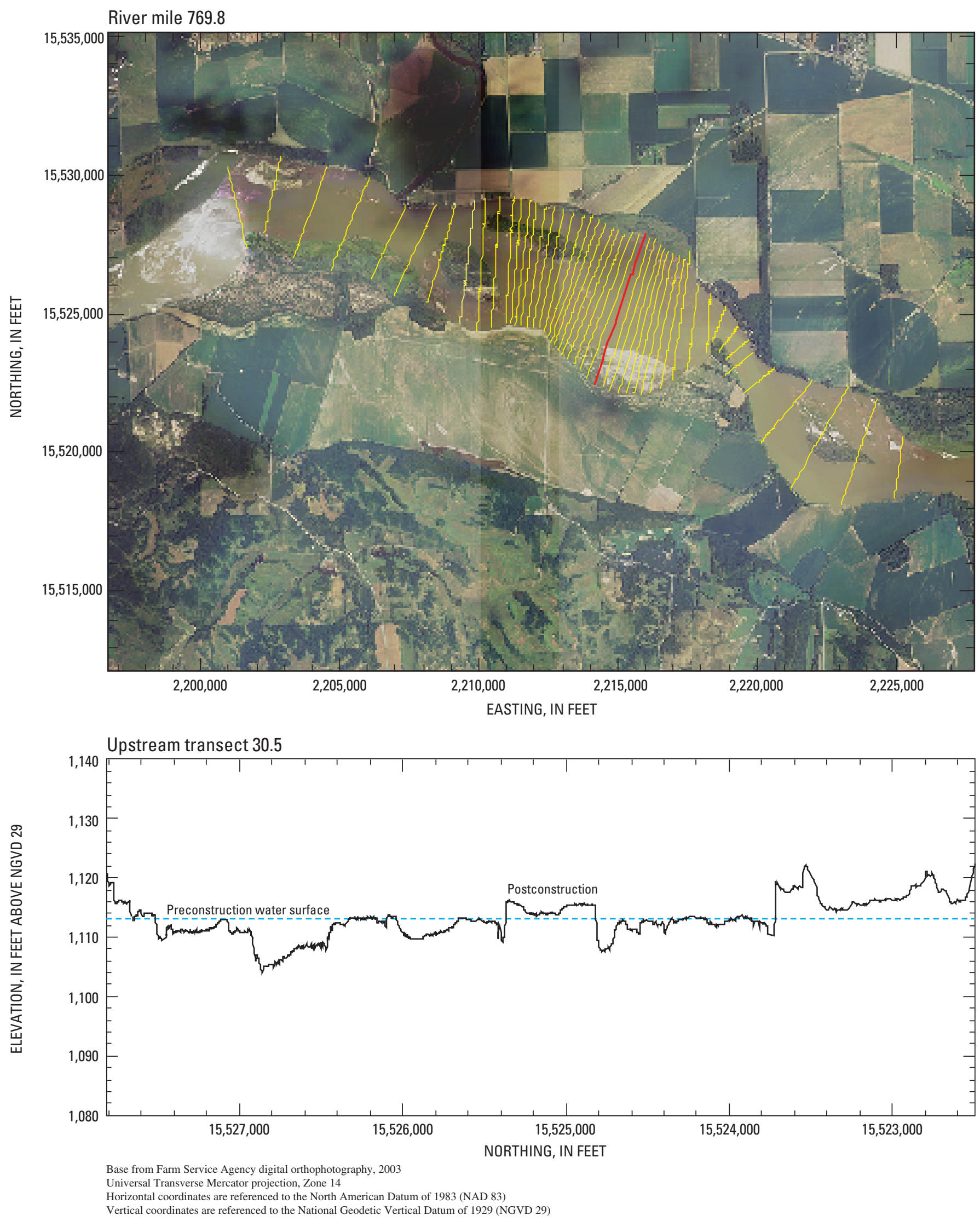

Figure A82. Location and cross section for upstream transect 30.5. 

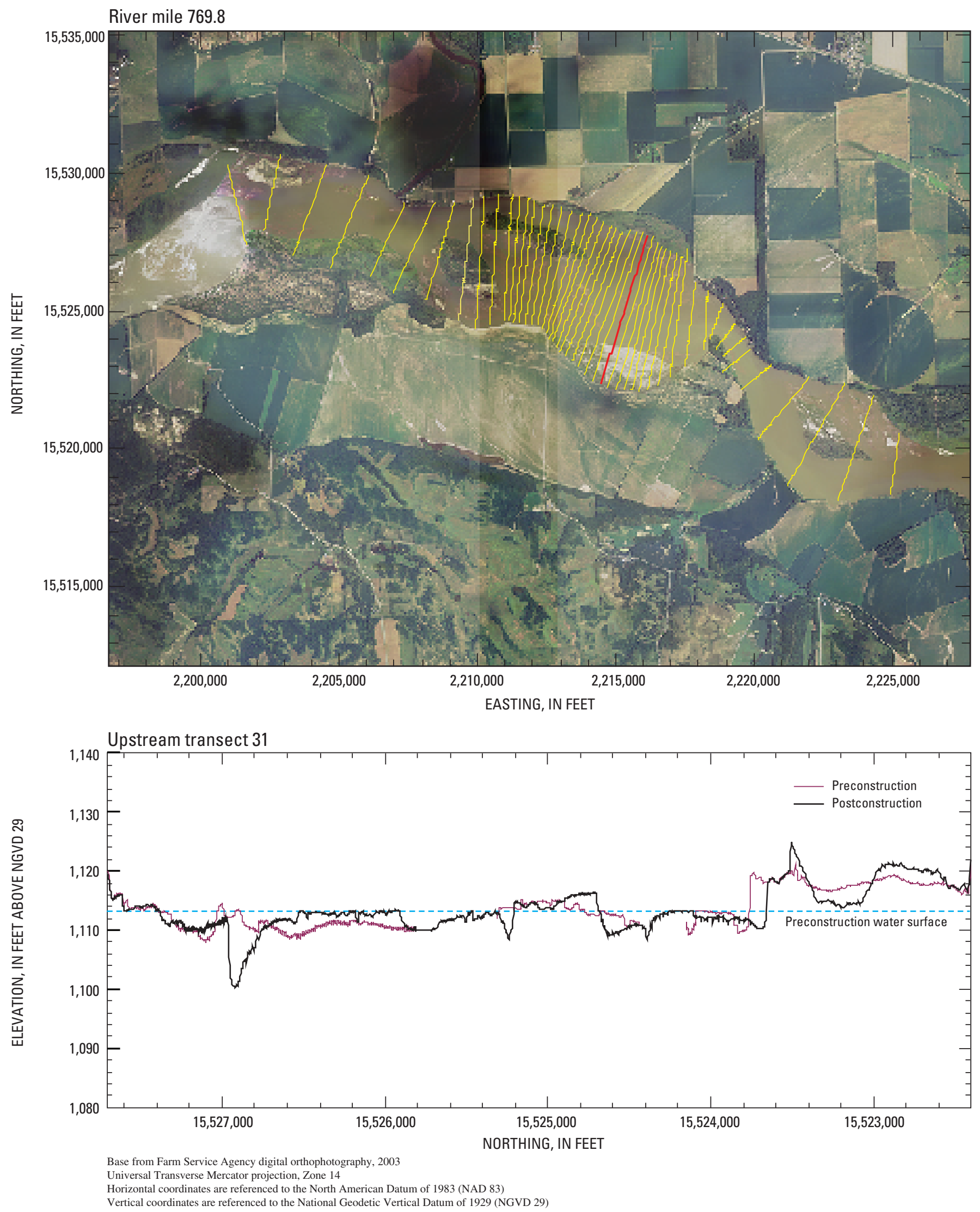

Figure A83. Location and cross section for upstream transect 31. 

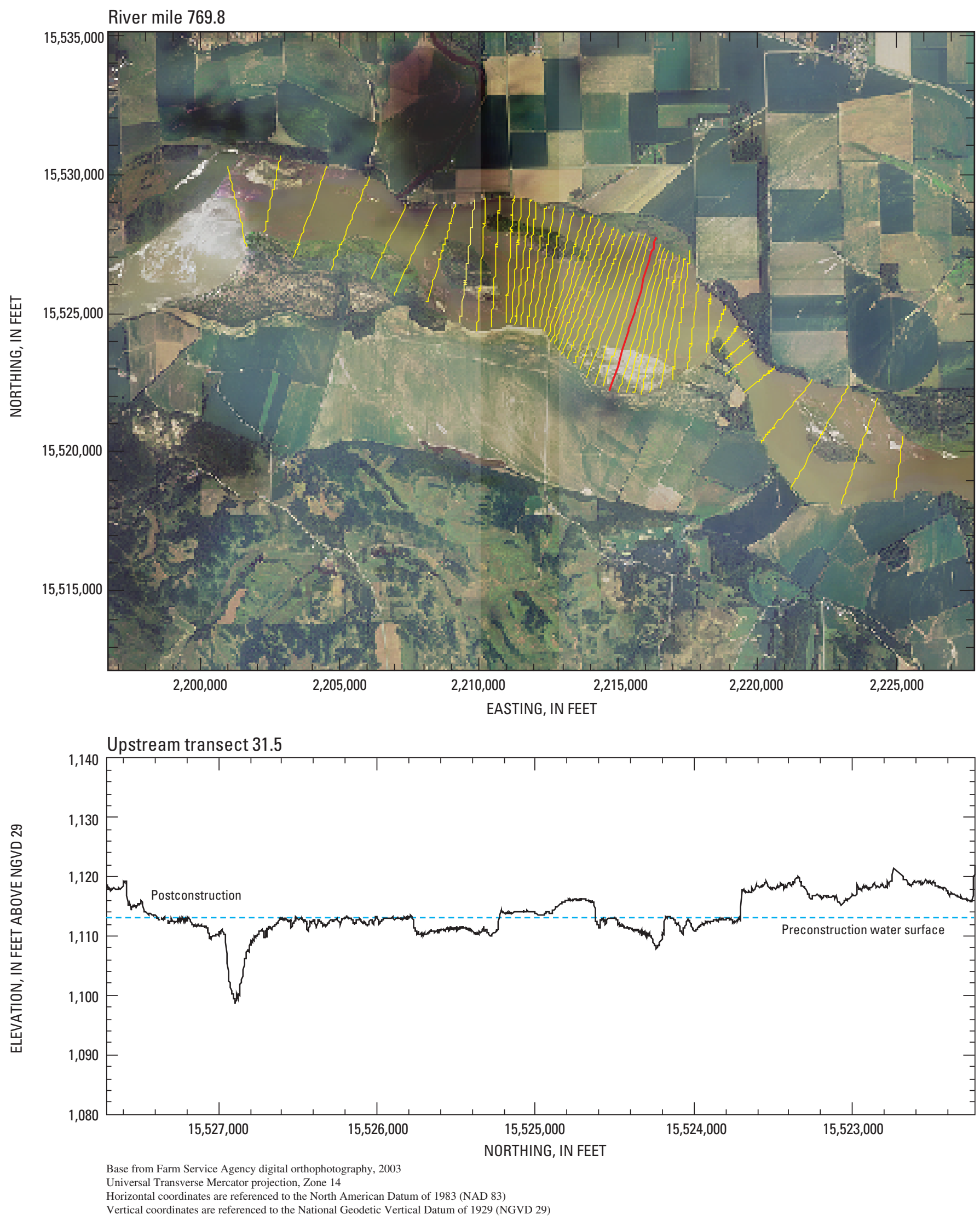

Figure A84. Location and cross section for upstream transect 31.5. 

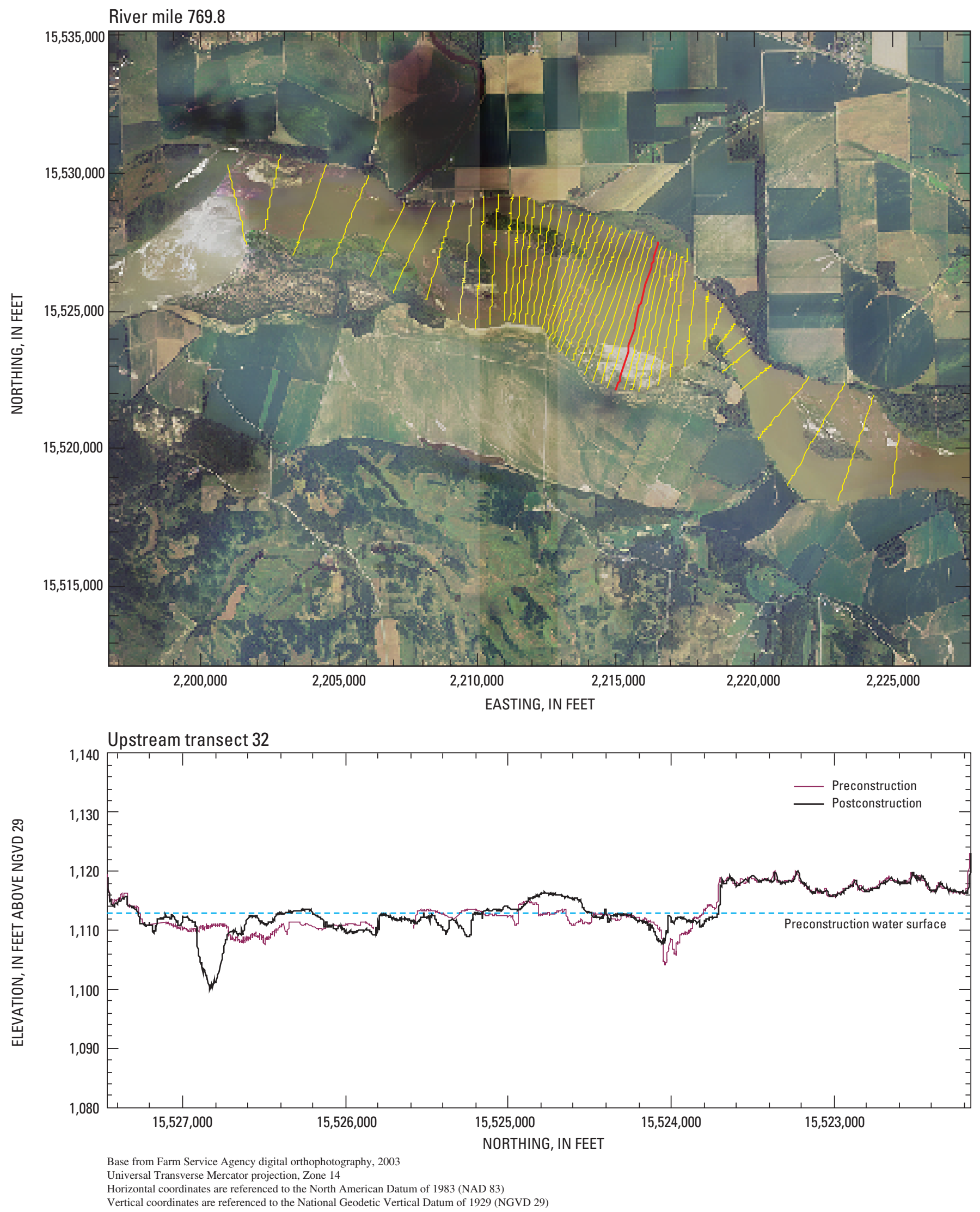

Figure A85. Location and cross section for upstream transect 32. 

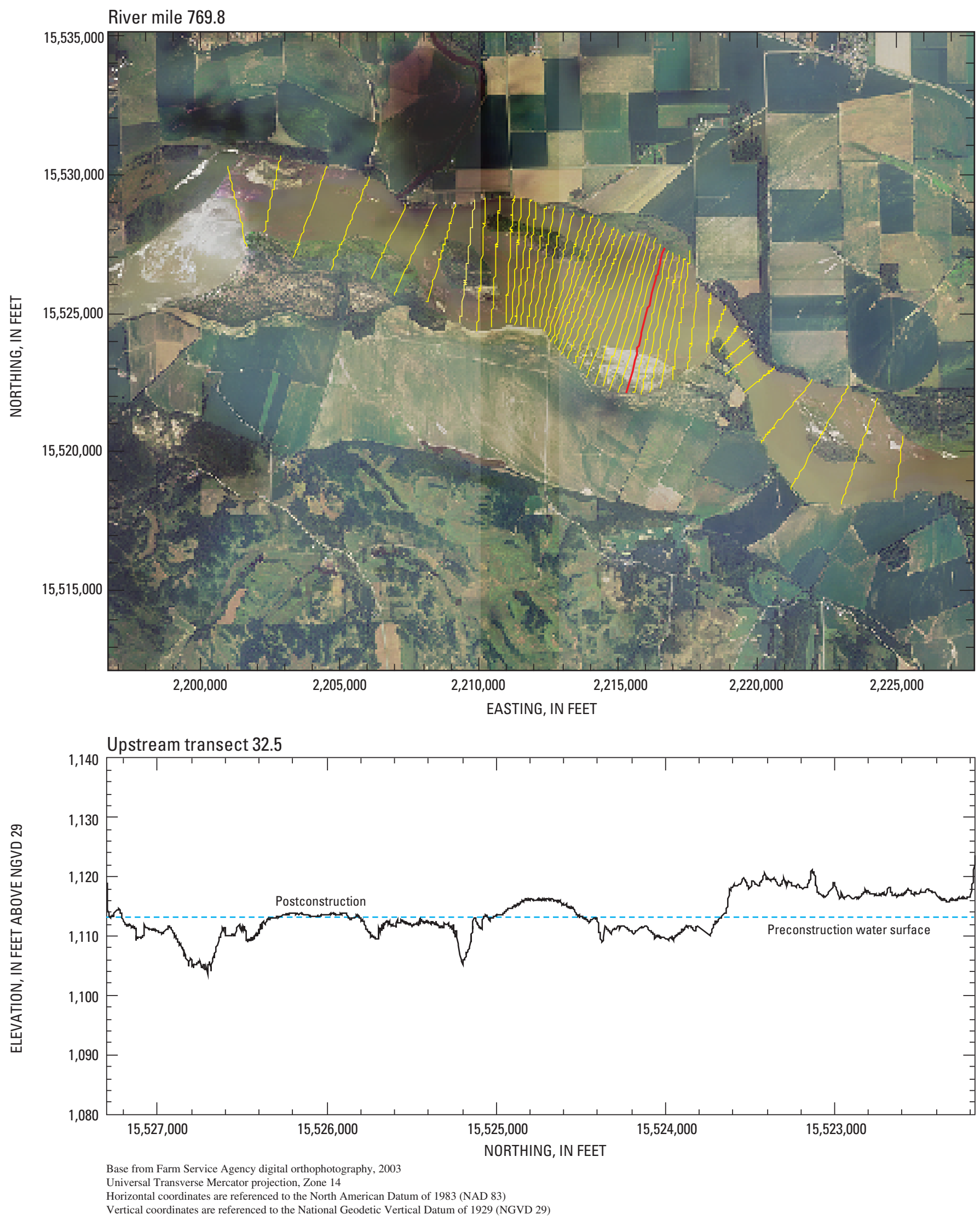

Figure A86. Location and cross section for upstream transect 32.5. 

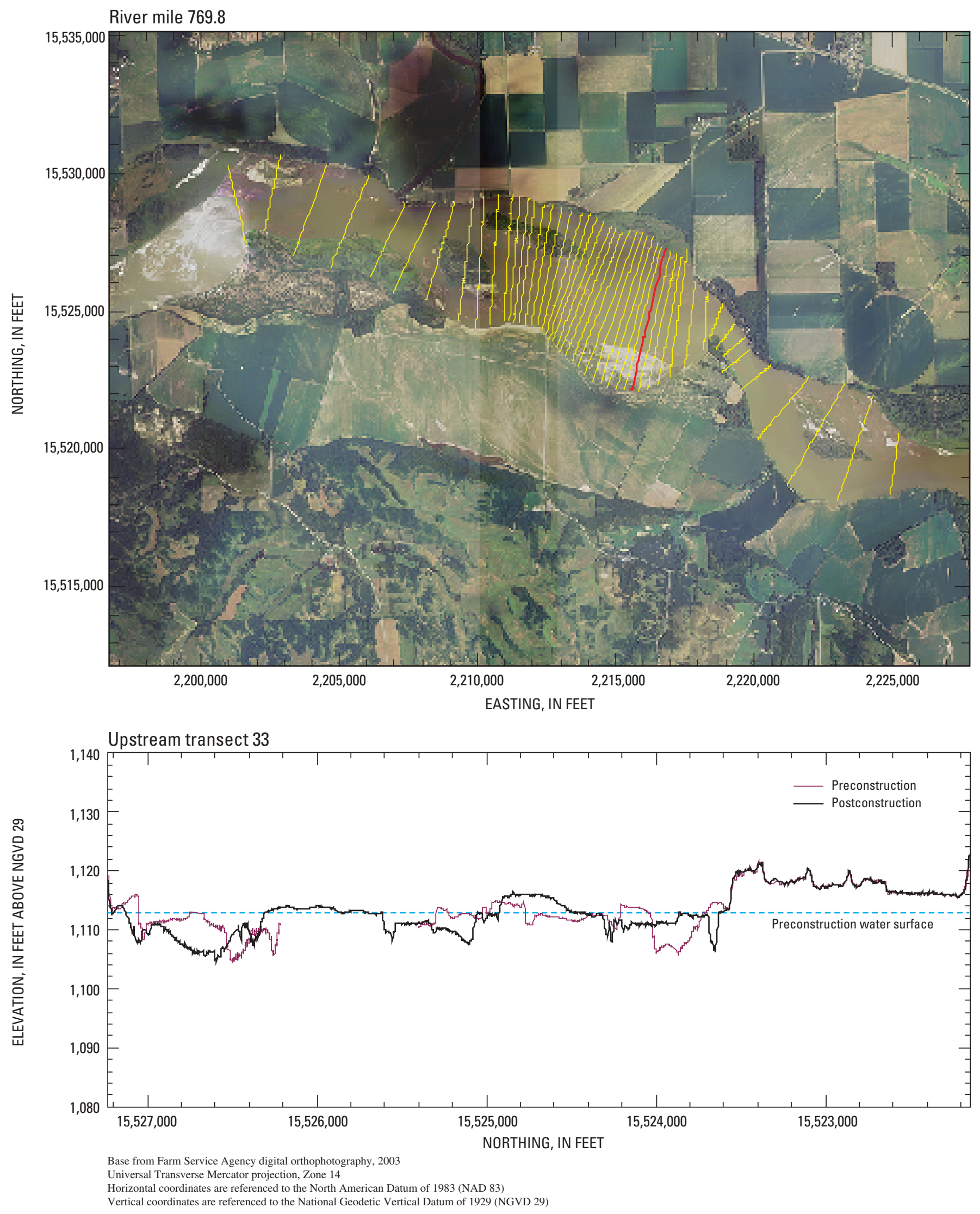

Figure A87. Location and cross section for upstream transect 33. 

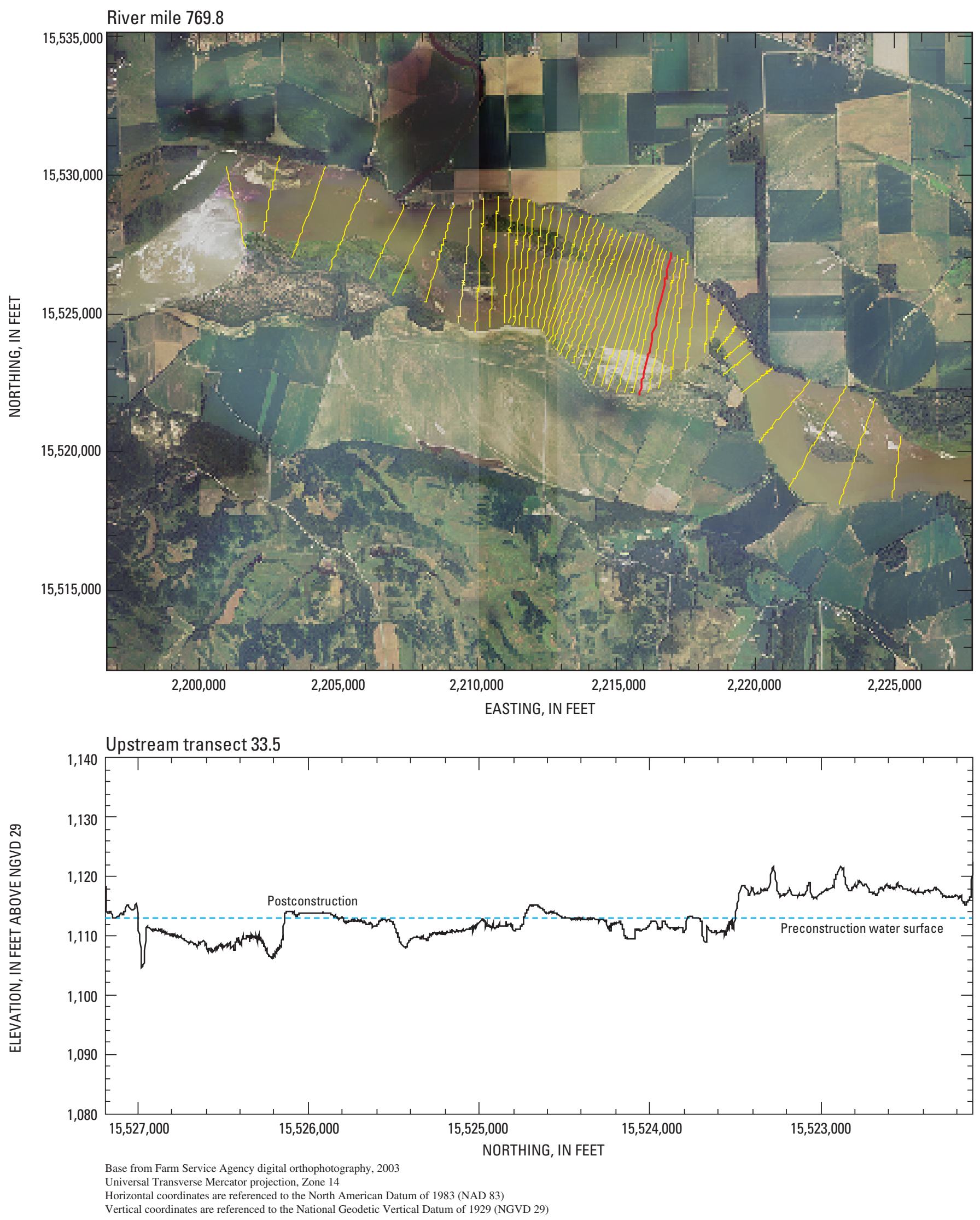

Figure A88. Location and cross section for upstream transect 33.5. 

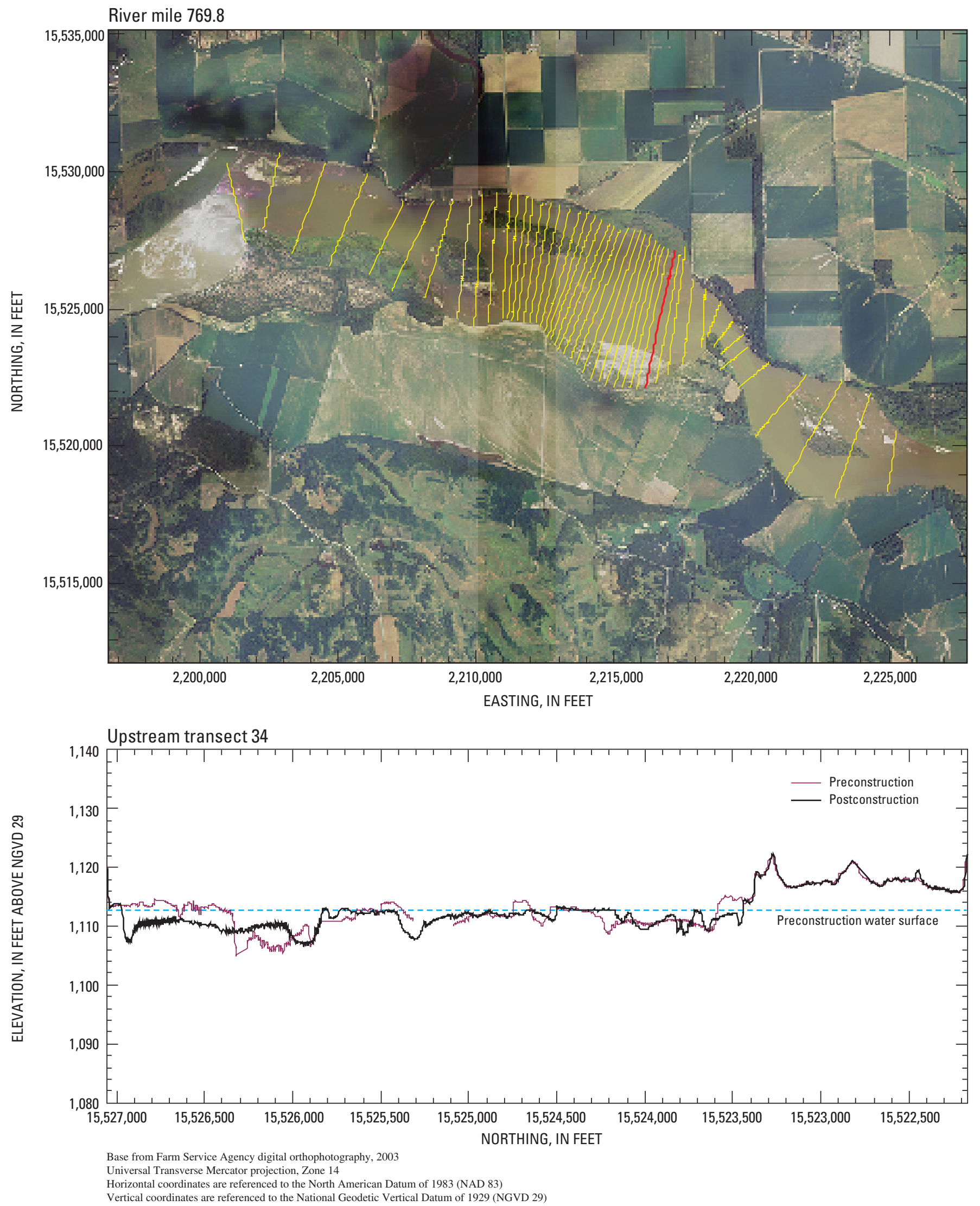

Figure A89. Location and cross section for upstream transect 34 . 

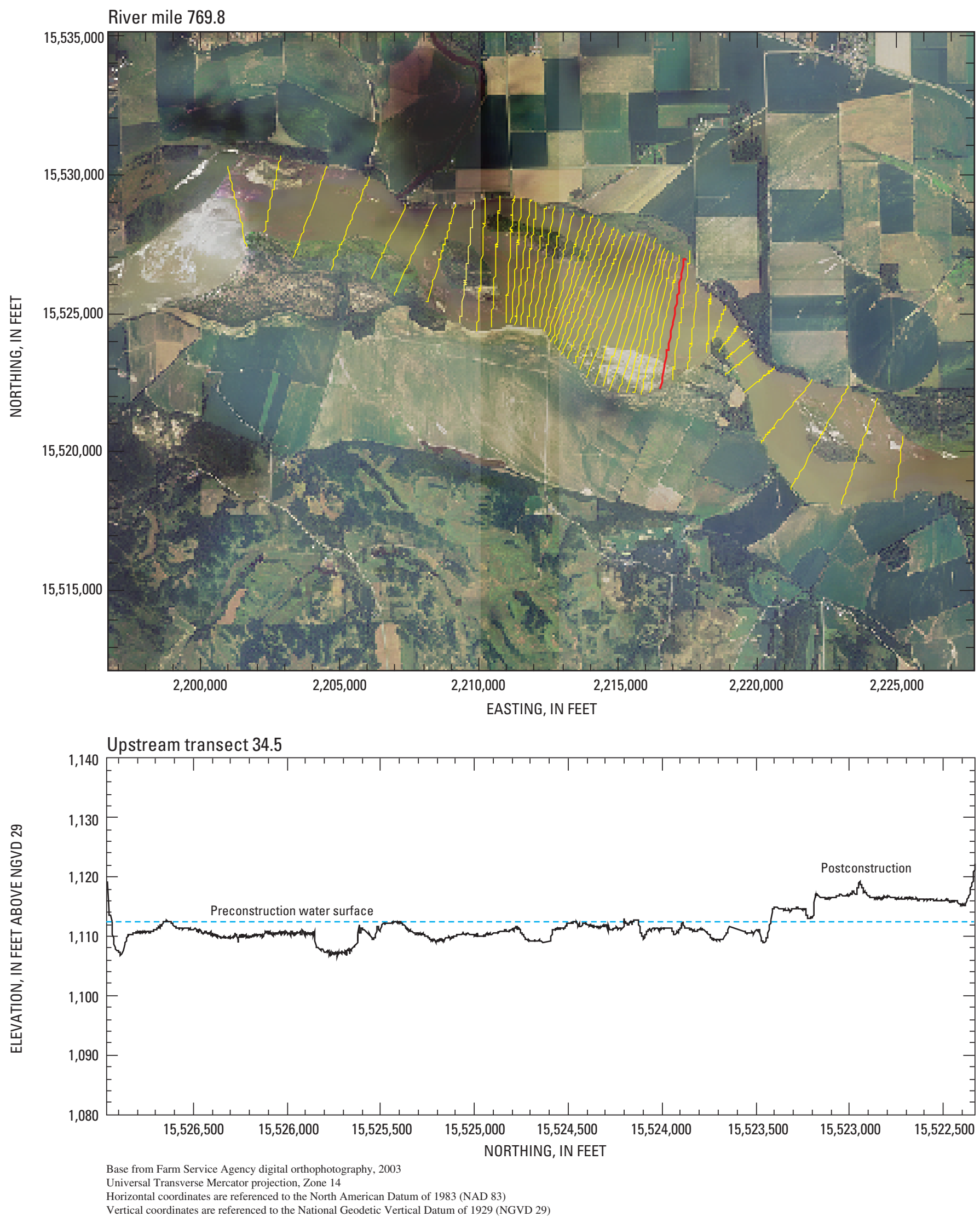

Figure A90. Location and cross section for upstream transect 34.5. 

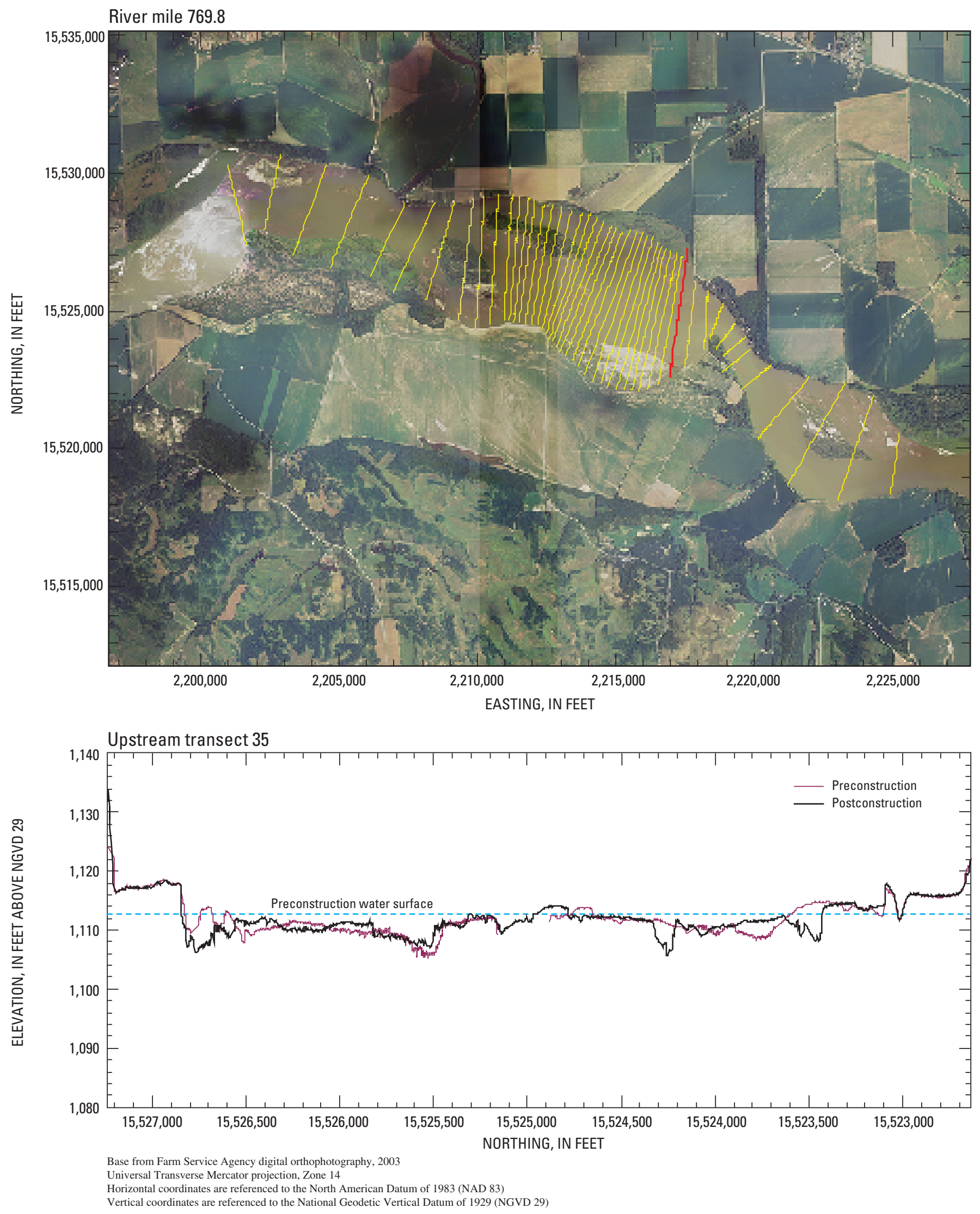

Figure A91. Location and cross section for upstream transect 35. 

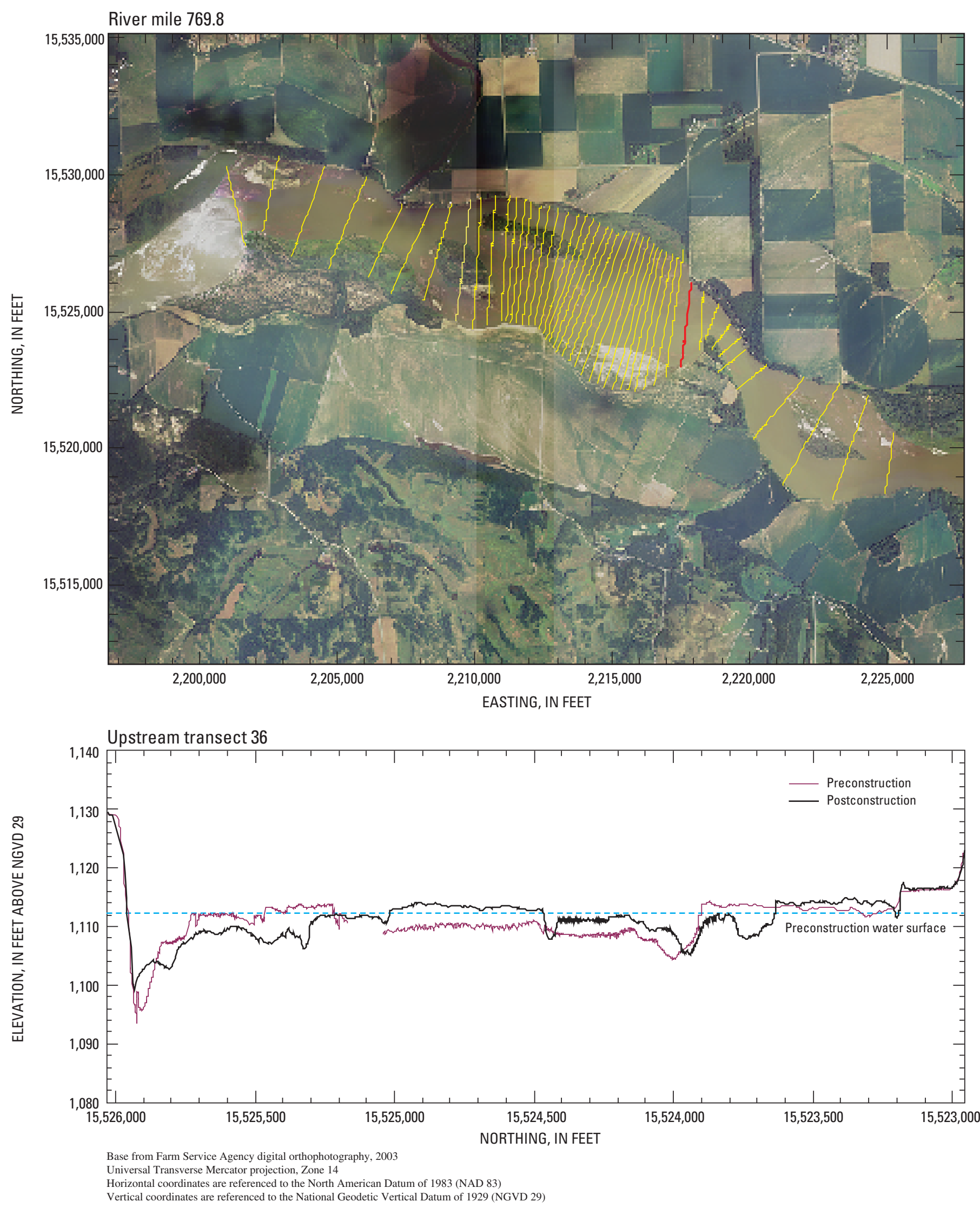

Figure A92. Location and cross section for upstream transect 36 . 

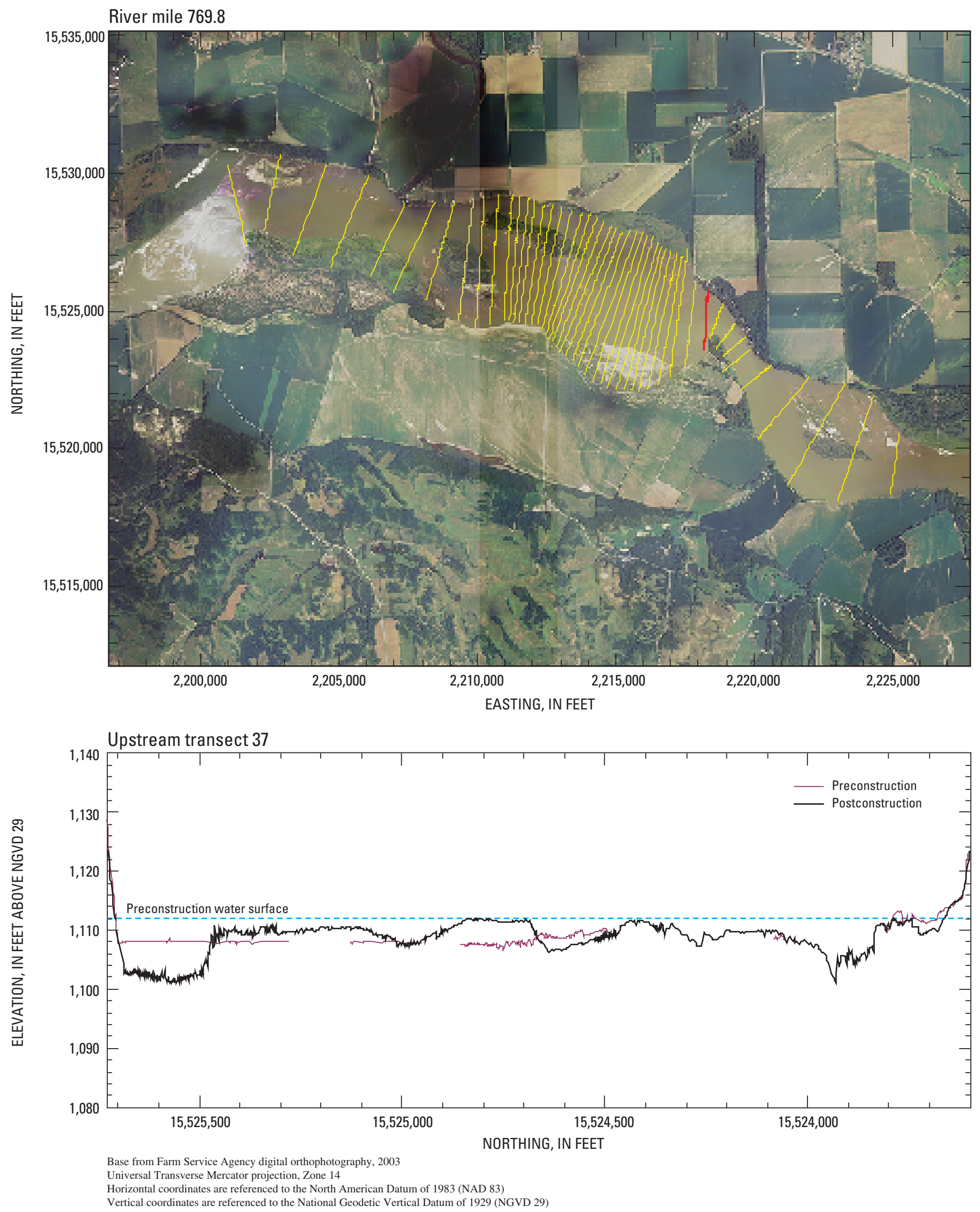

Figure A93. Location and cross section for upstream transect 37. 

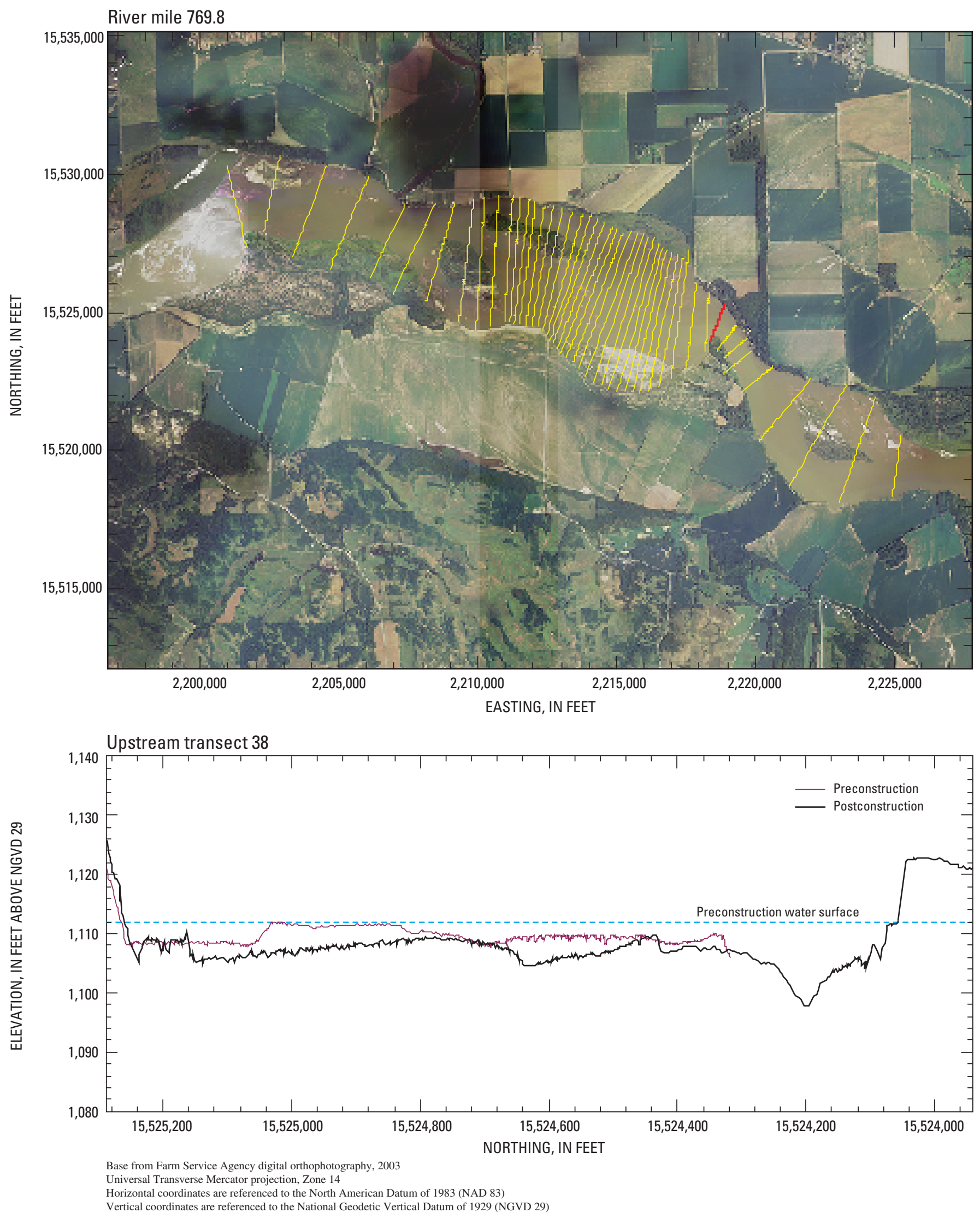

Figure A94. Location and cross section for upstream transect 38. 

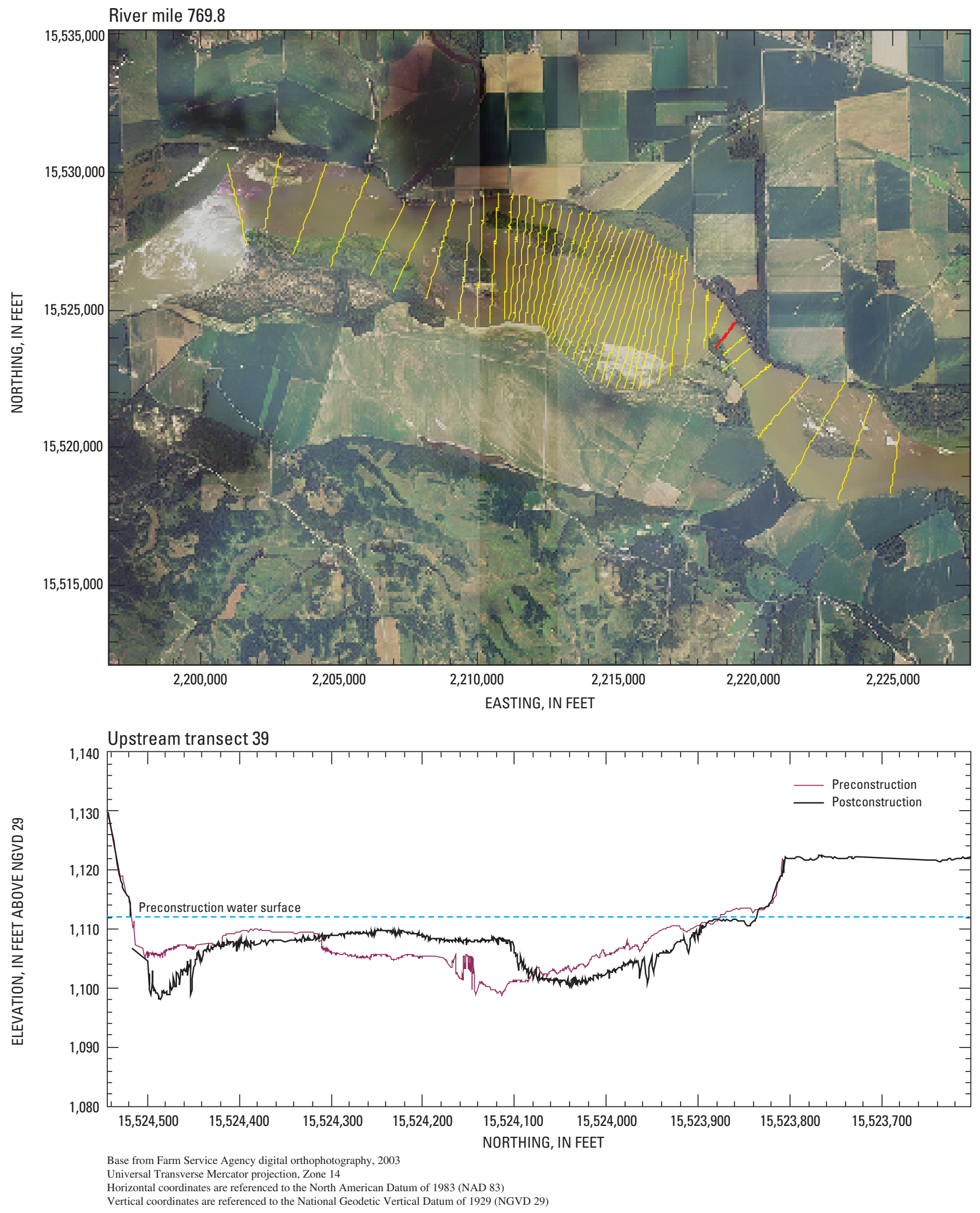

Figure A95. Location and cross section for upstream transect 39 . 

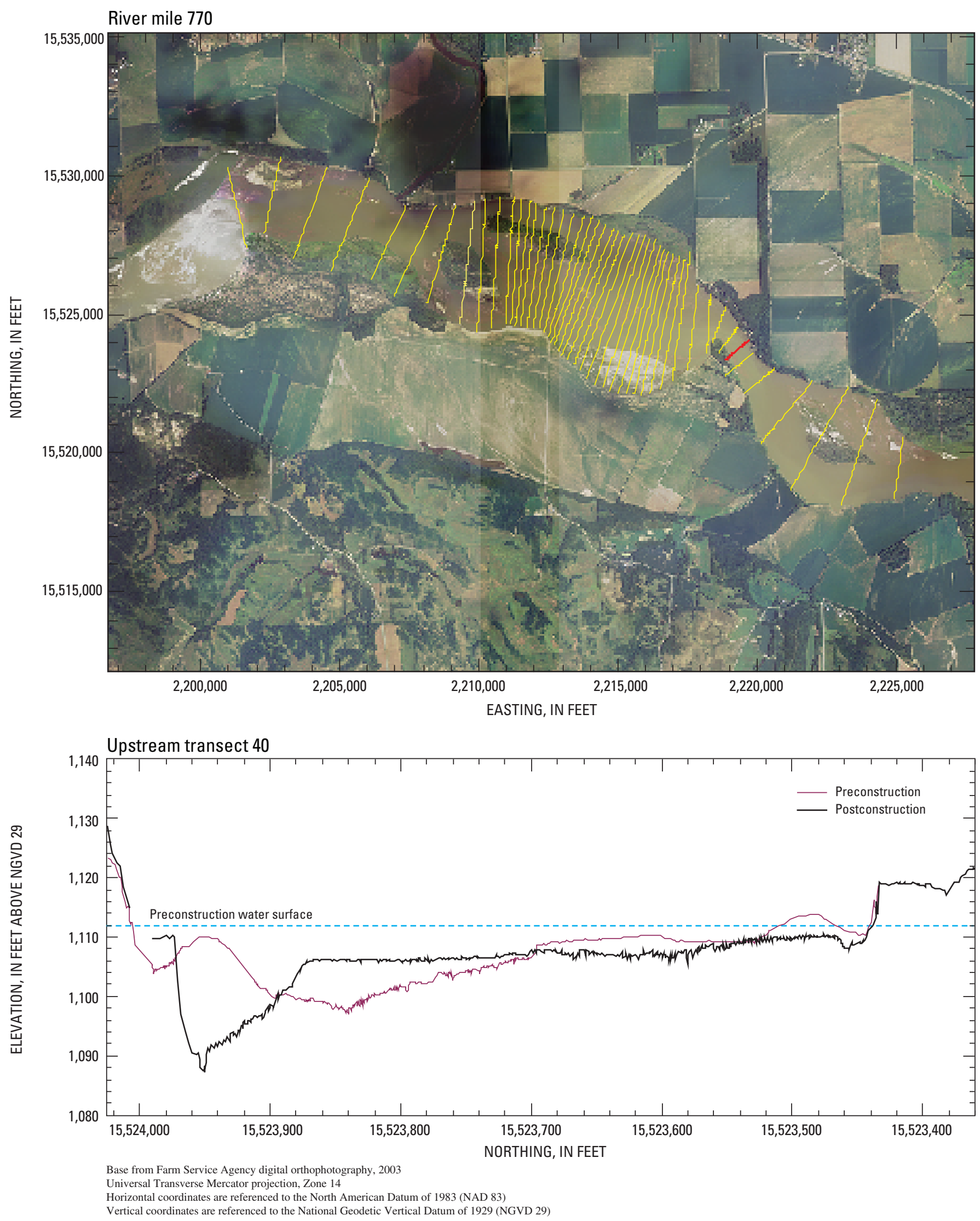

Figure A96. Location and cross section for upstream transect 40. 

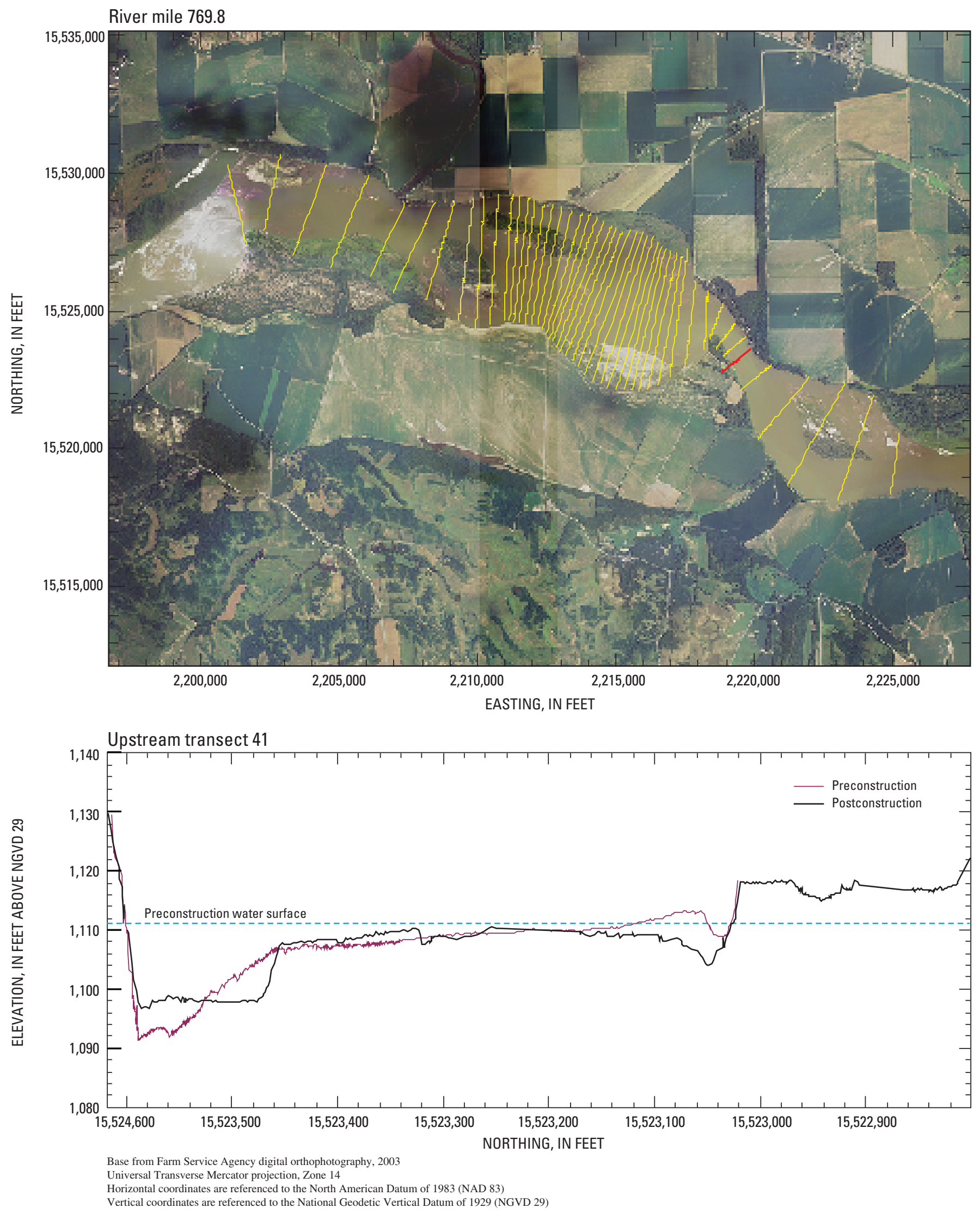

Figure A97. Location and cross section for upstream transect 41. 

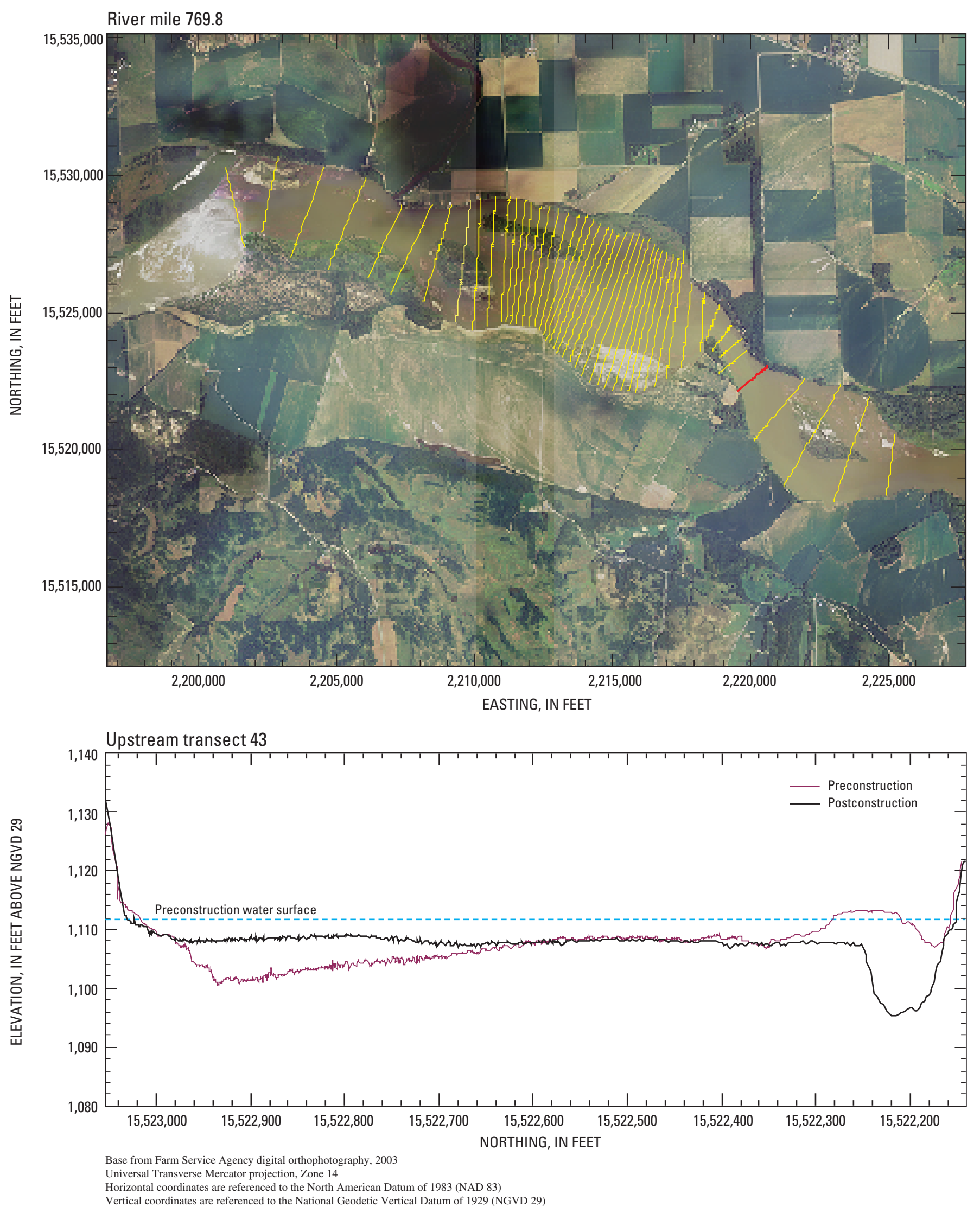

Figure A98. Location and cross section for upstream transect 43. 

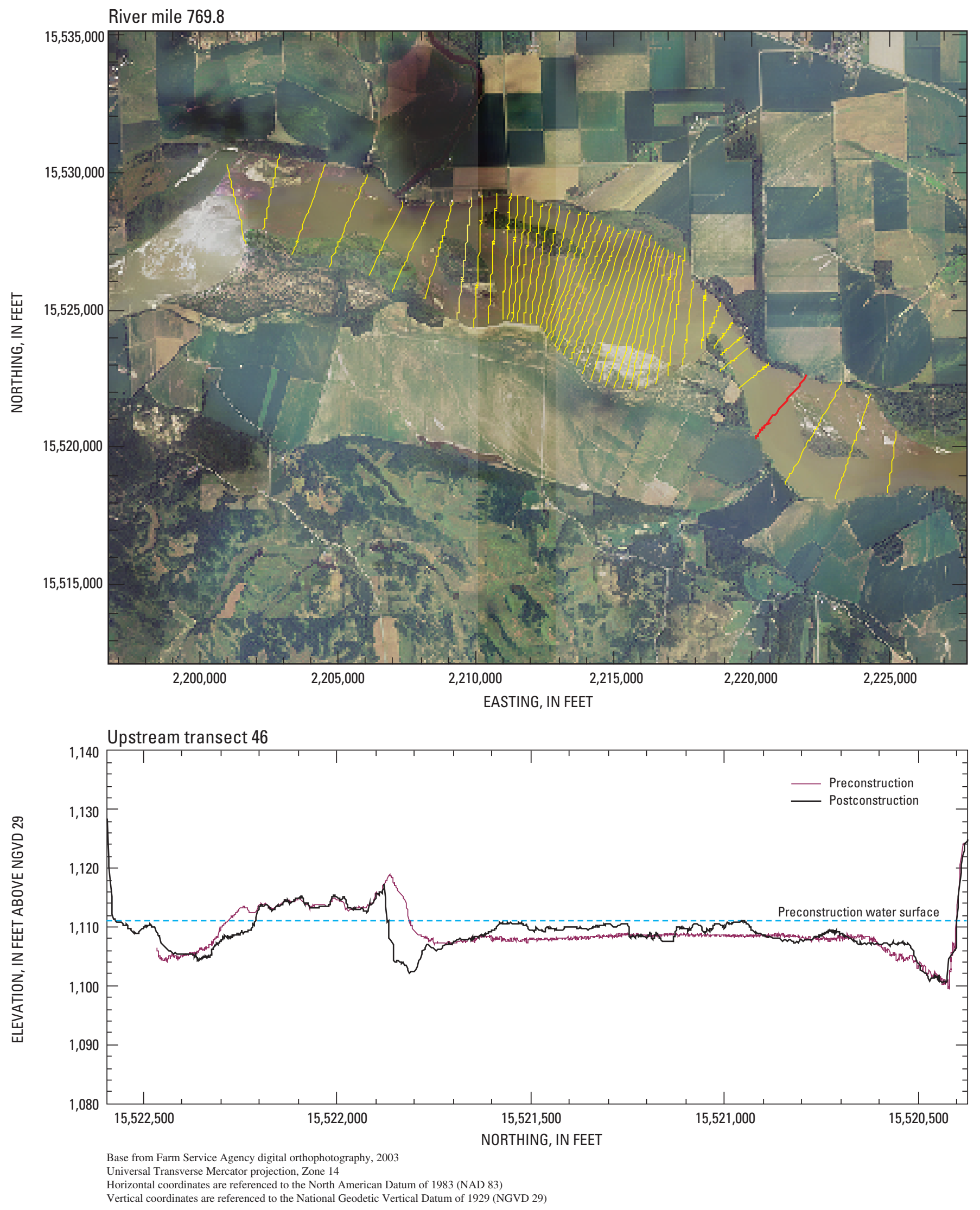

Figure A99. Location and cross section for upstream transect 46. 

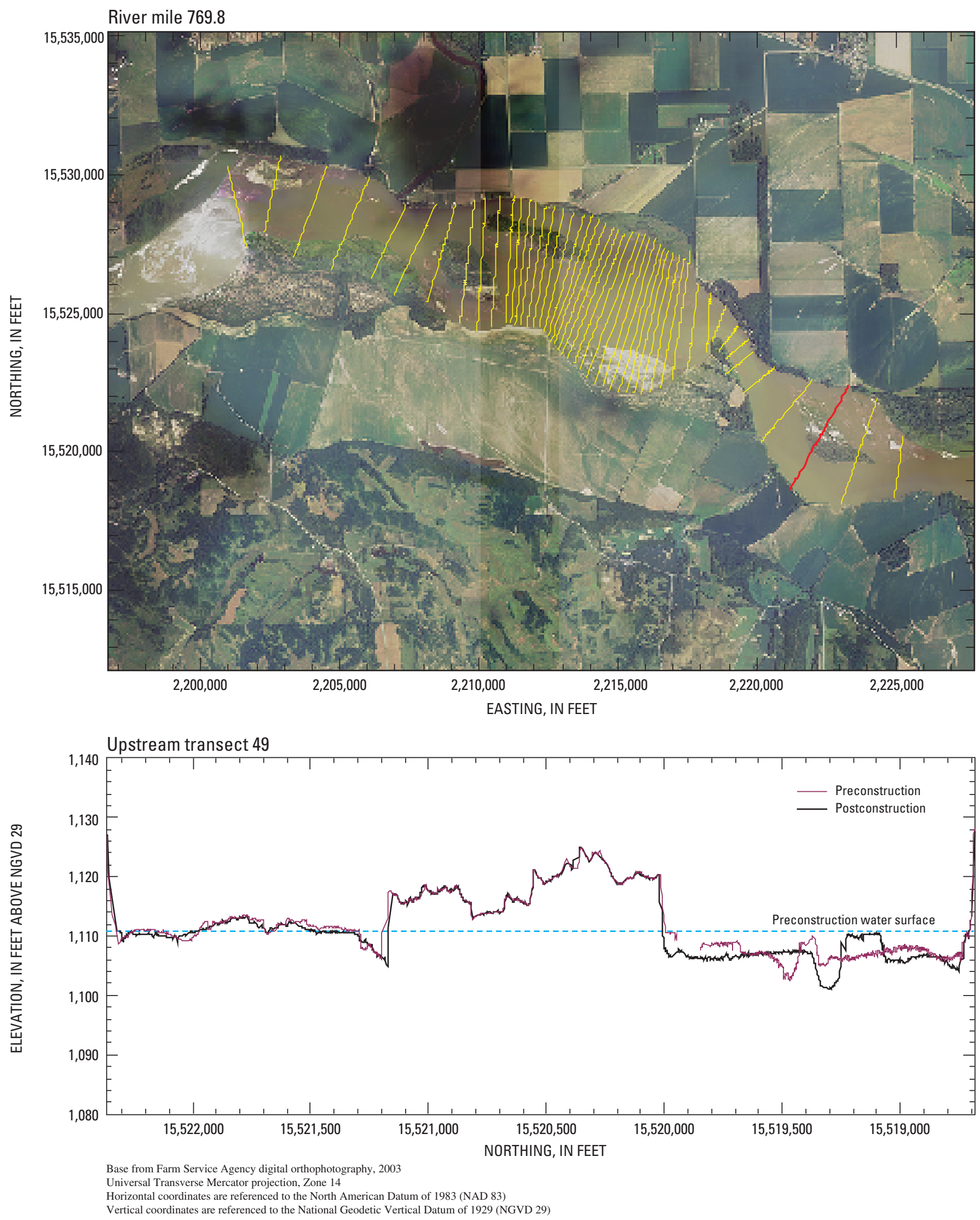

Figure A100. Location and cross section for upstream transect 49. 

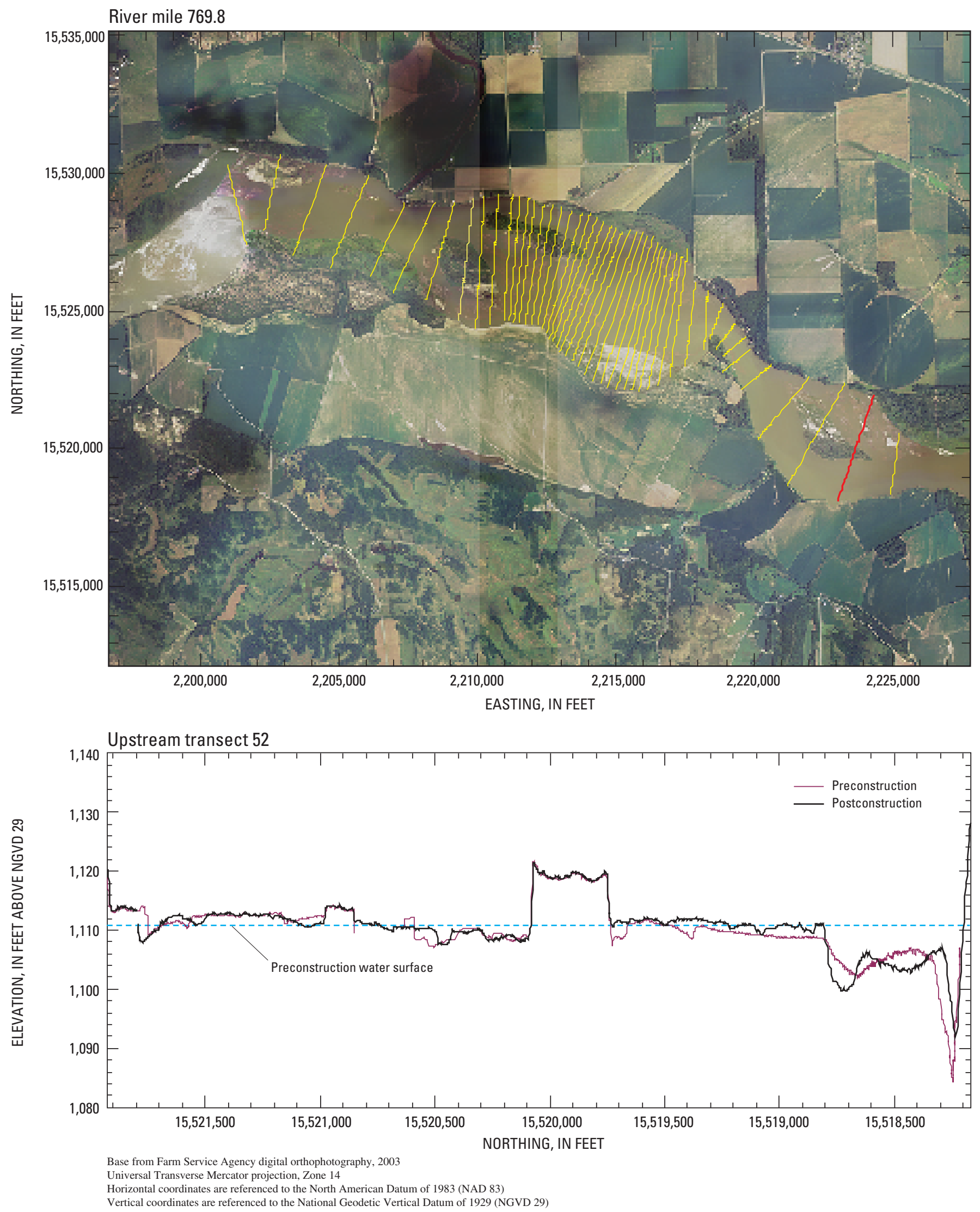

Figure A101. Location and cross section for upstream transect 52. 

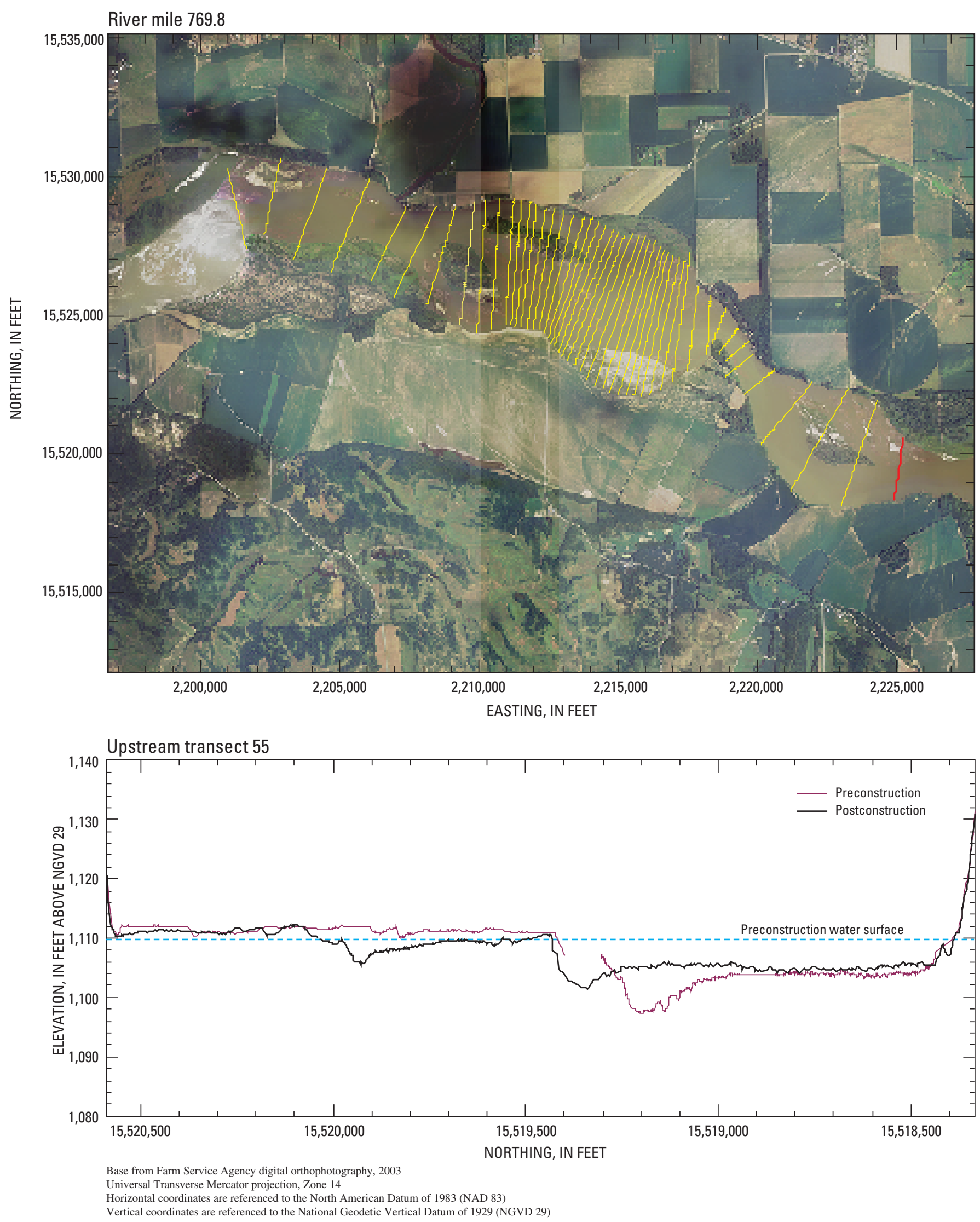

Figure A102. Location and cross section for upstream transect 55. 


\section{Section B. Color-Shaded Water-Velocity Profiles of Upstream Study Area}

A series of figures is presented for the velocity profiles collected with the ADCP (figs. B1-B47) for the upstream study area. The top part of each figure in this series shows the location of a highlighted transect, and the lower part shows the velocity profile of that transect 

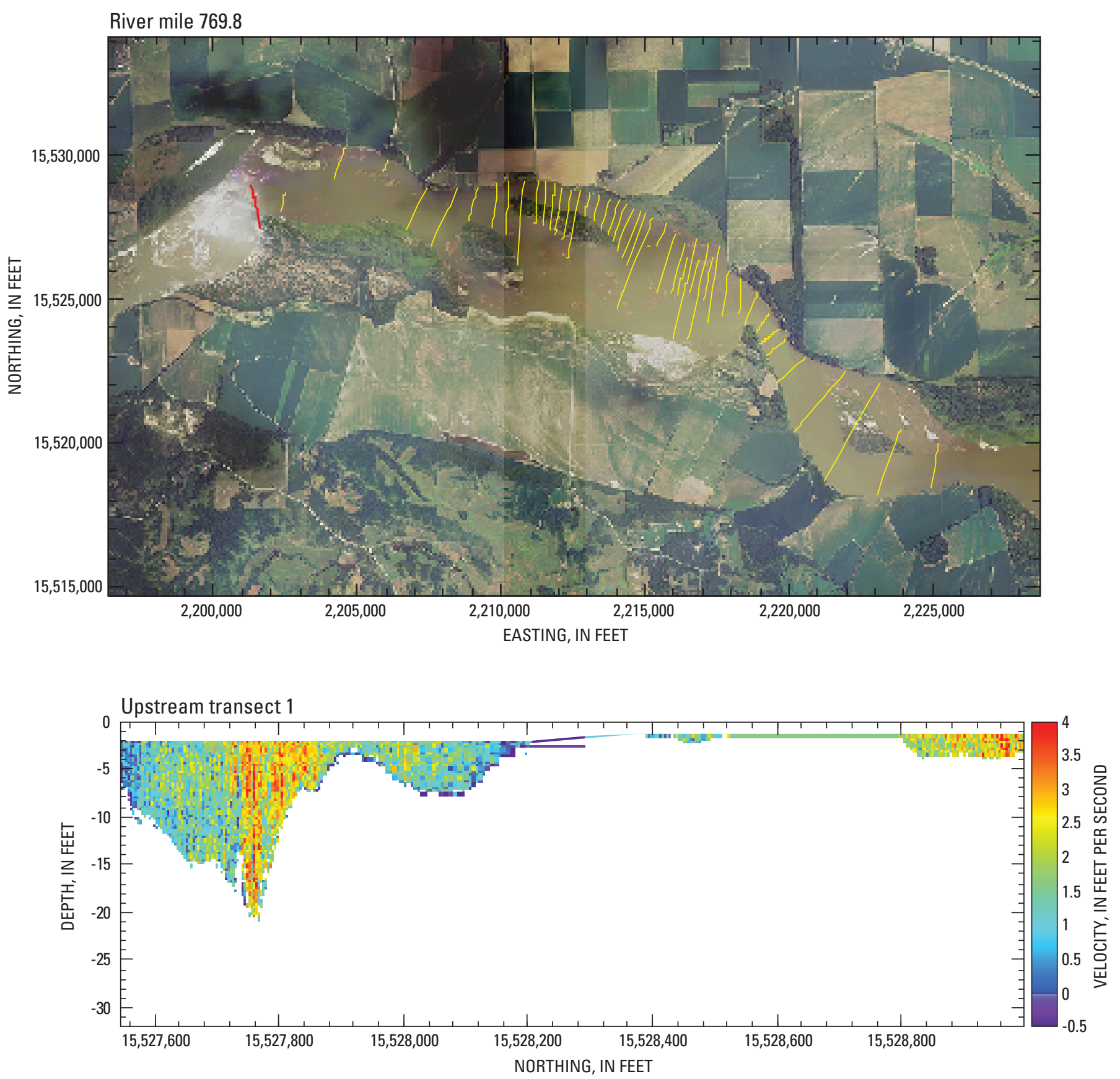

Base from Farm Service Agency digital orthophotography, 2003

Universal Transverse Mercator projection, Zone 14

Horizontal coordinates are referenced to the North American Datum of 1983 (NAD 83)

Figure B1. Location and velocity profile for upstream transect 1. 

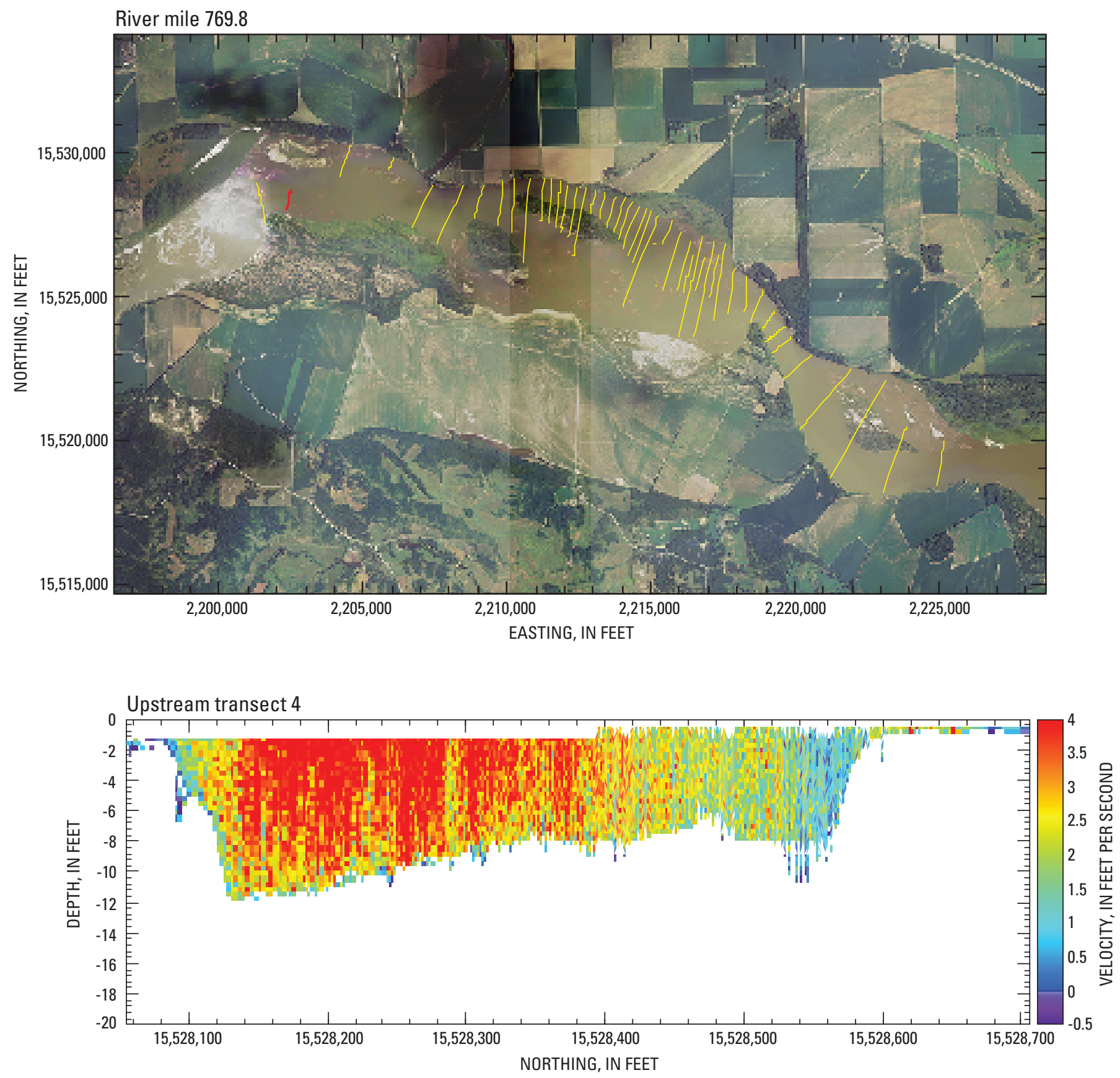

Base from Farm Service Agency digital orthophotography, 2003

Universal Transverse Mercator projection, Zone 14

Horizontal coordinates are referenced to the North American Datum of 1983 (NAD 83)

Figure B2. Location and velocity profile for upstream transect 4. 

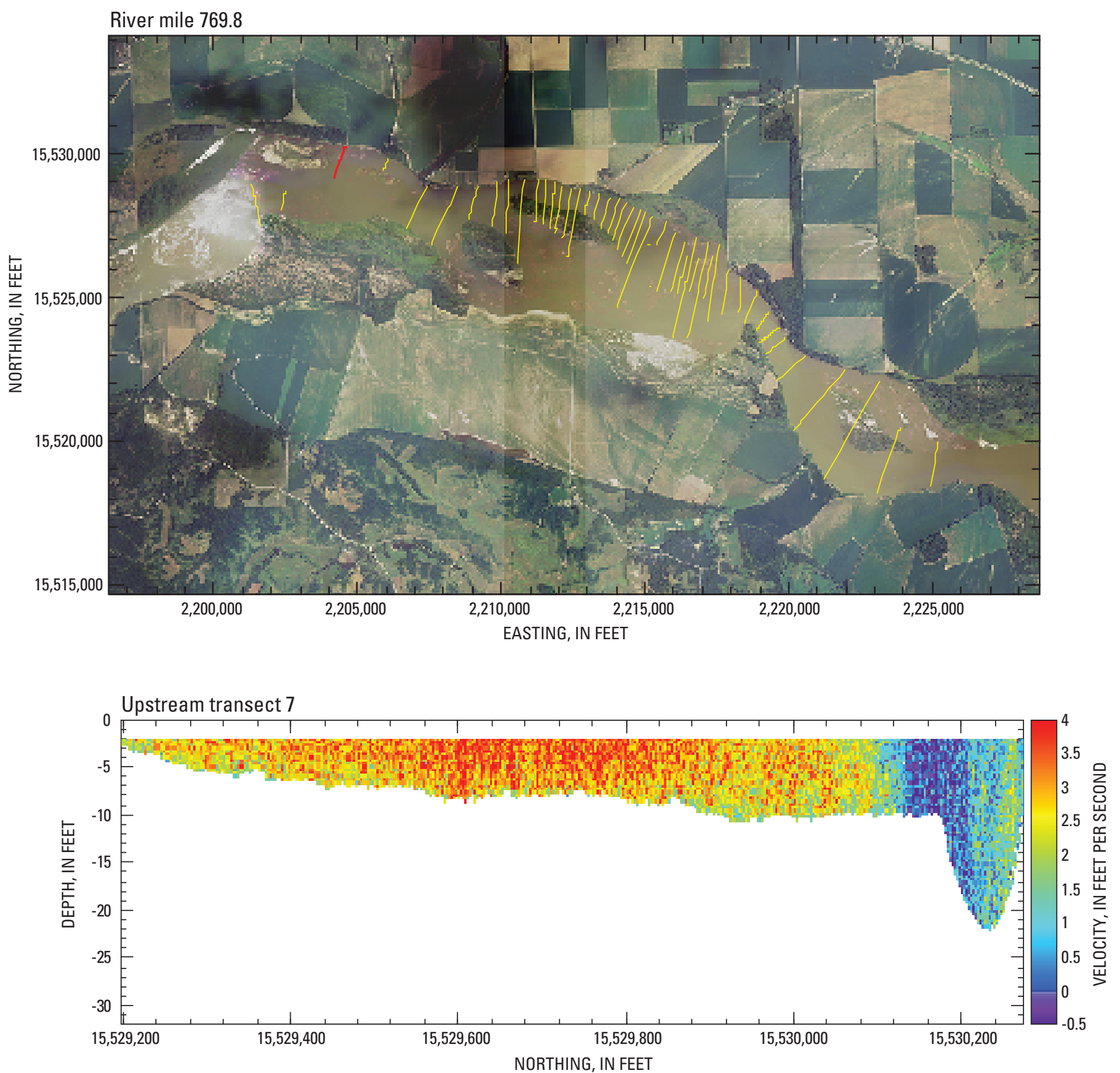

Base from Farm Service Agency digital orthophotography, 2003

Universal Transverse Mercator projection, Zone 14

Horizontal coordinates are referenced to the North American Datum of 1983 (NAD 83)

Figure B3. Location and velocity profile for upstream transect 7. 

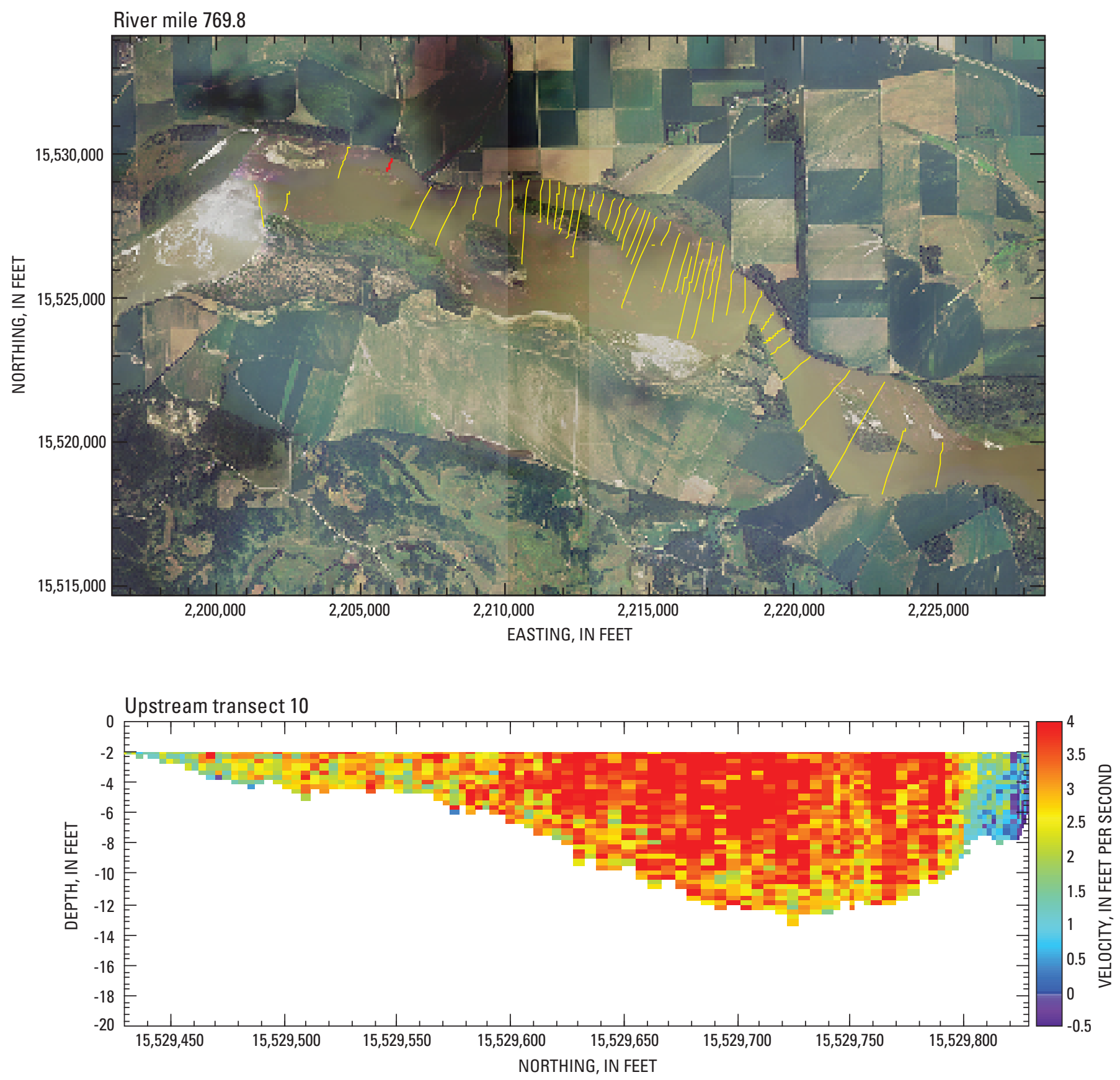

Base from Farm Service Agency digital orthophotography, 2003

Universal Transverse Mercator projection, Zone 14

Horizontal coordinates are referenced to the North American Datum of 1983 (NAD 83)

Figure B4. Location and velocity profile for upstream transect 10. 

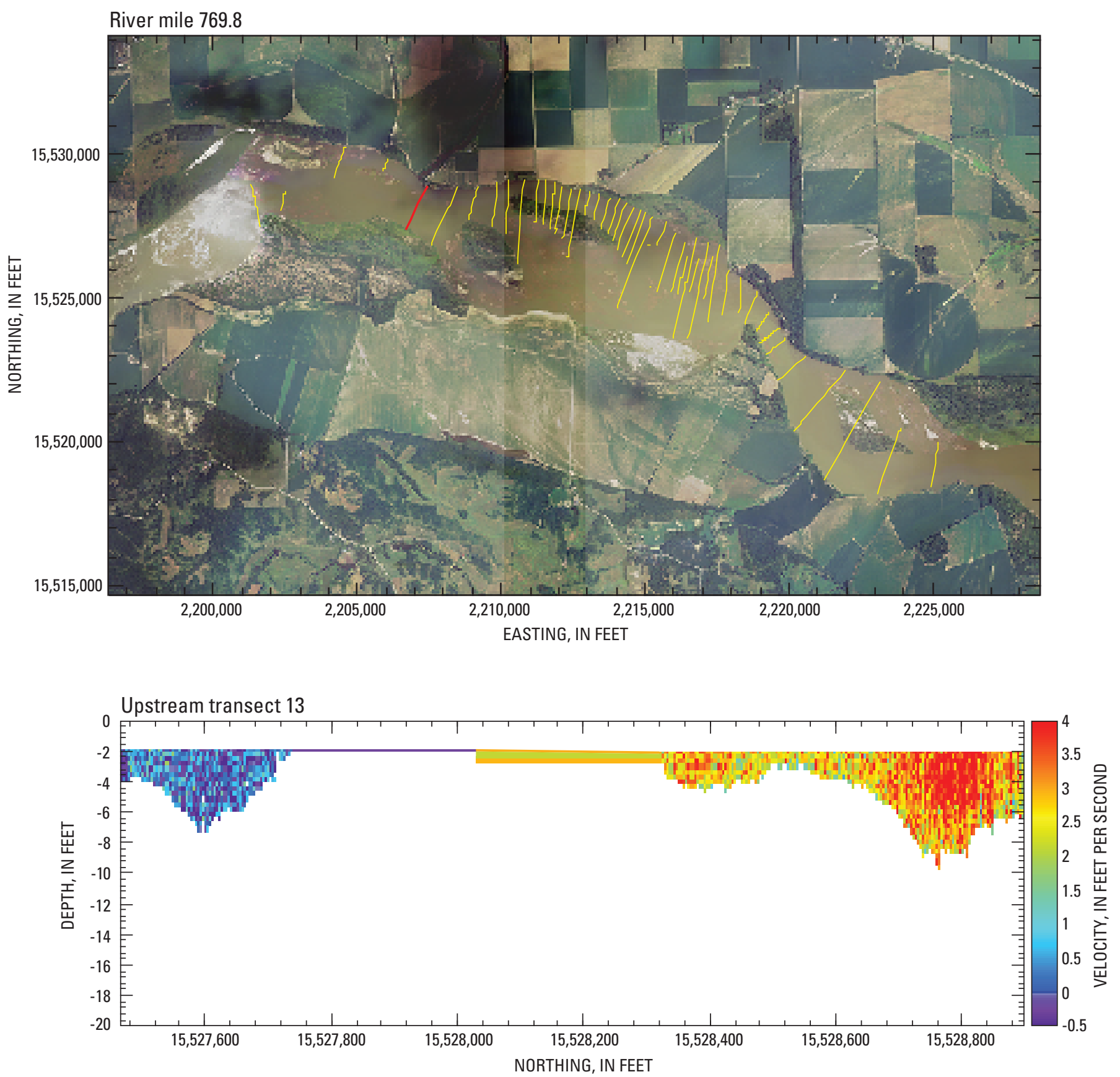

Base from Farm Service Agency digital orthophotography, 2003

Universal Transverse Mercator projection, Zone 14

Horizontal coordinates are referenced to the North American Datum of 1983 (NAD 83)

Figure B5. Location and velocity profile for upstream transect 13 . 

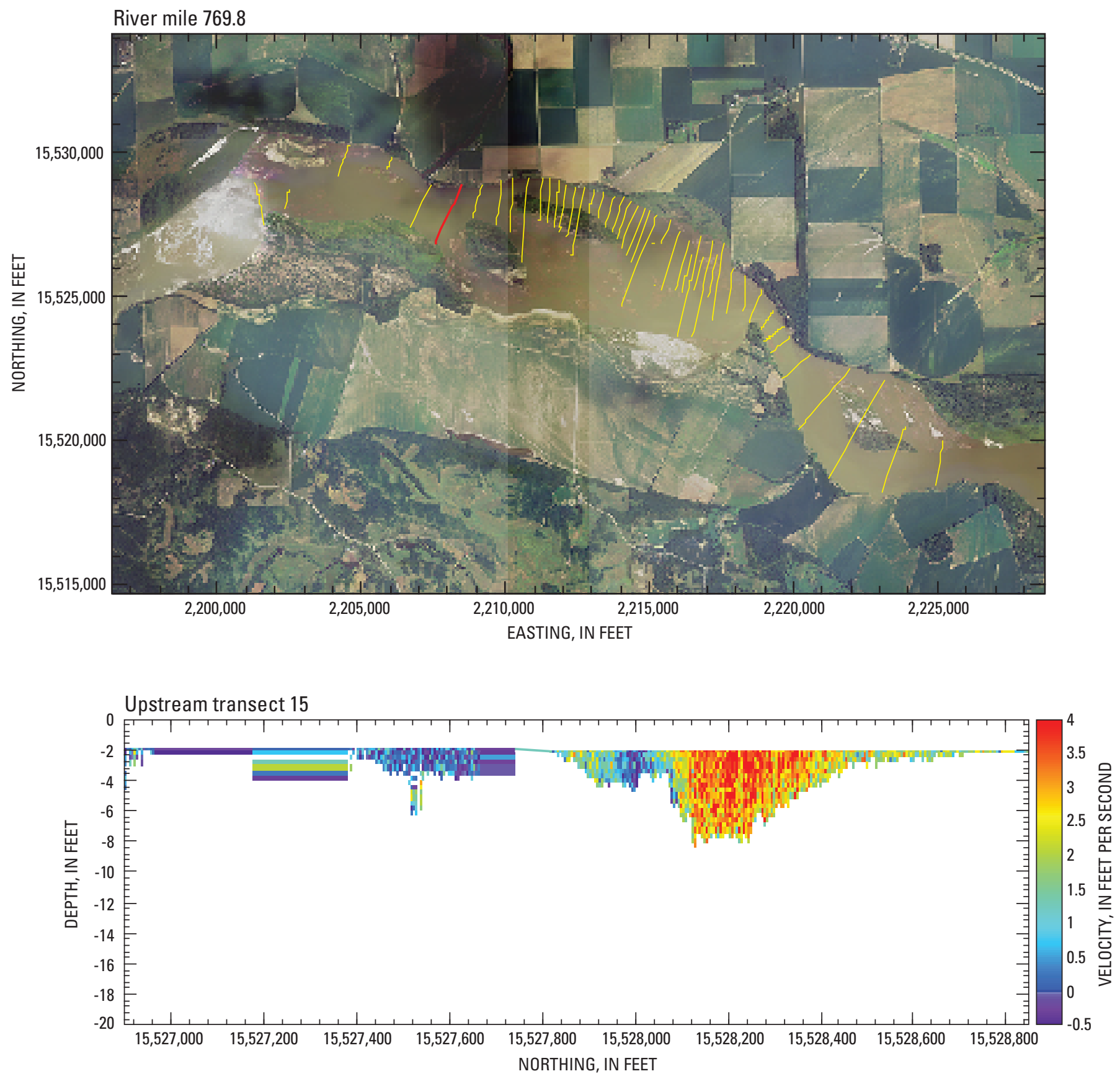

Base from Farm Service Agency digital orthophotography, 2003

Universal Transverse Mercator projection, Zone 14

Horizontal coordinates are referenced to the North American Datum of 1983 (NAD 83)

Figure B6. Location and velocity profile for upstream transect 15 . 

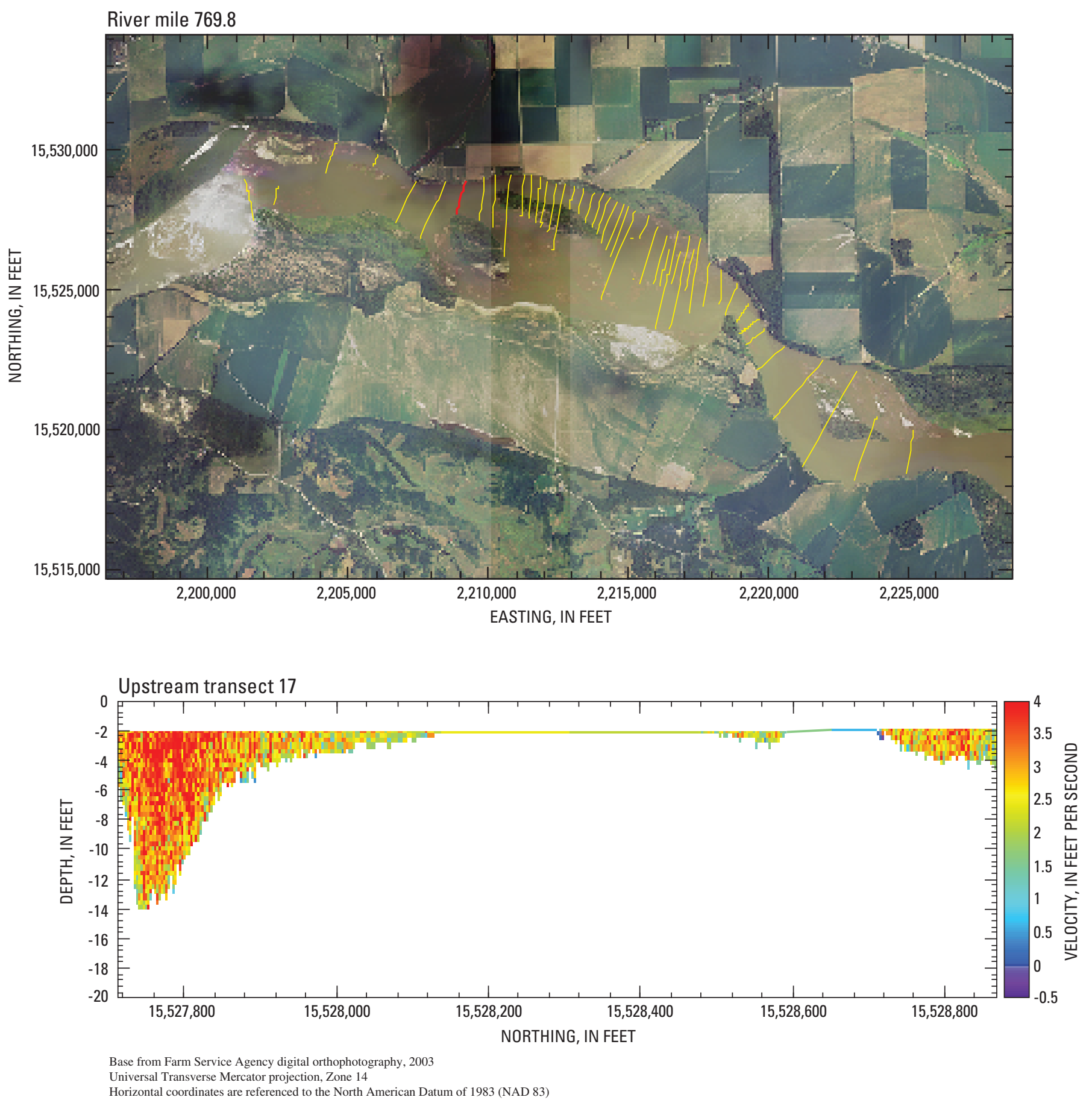

Figure B7. Location and velocity profile for upstream transect 17. 

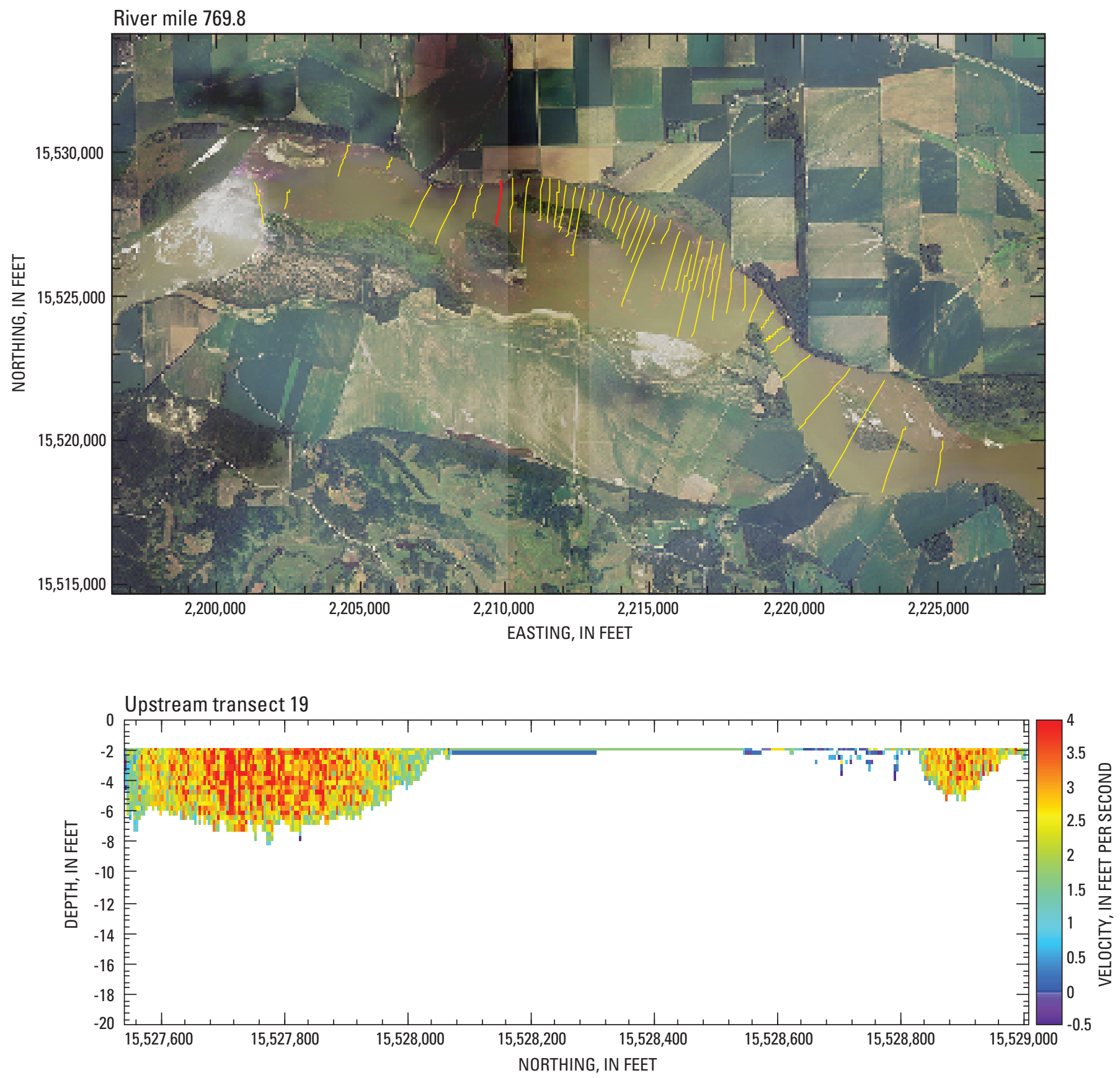

Base from Farm Service Agency digital orthophotography, 2003

Universal Transverse Mercator projection, Zone 14

Horizontal coordinates are referenced to the North American Datum of 1983 (NAD 83)

Figure B8. Location and velocity profile for upstream transect 19. 

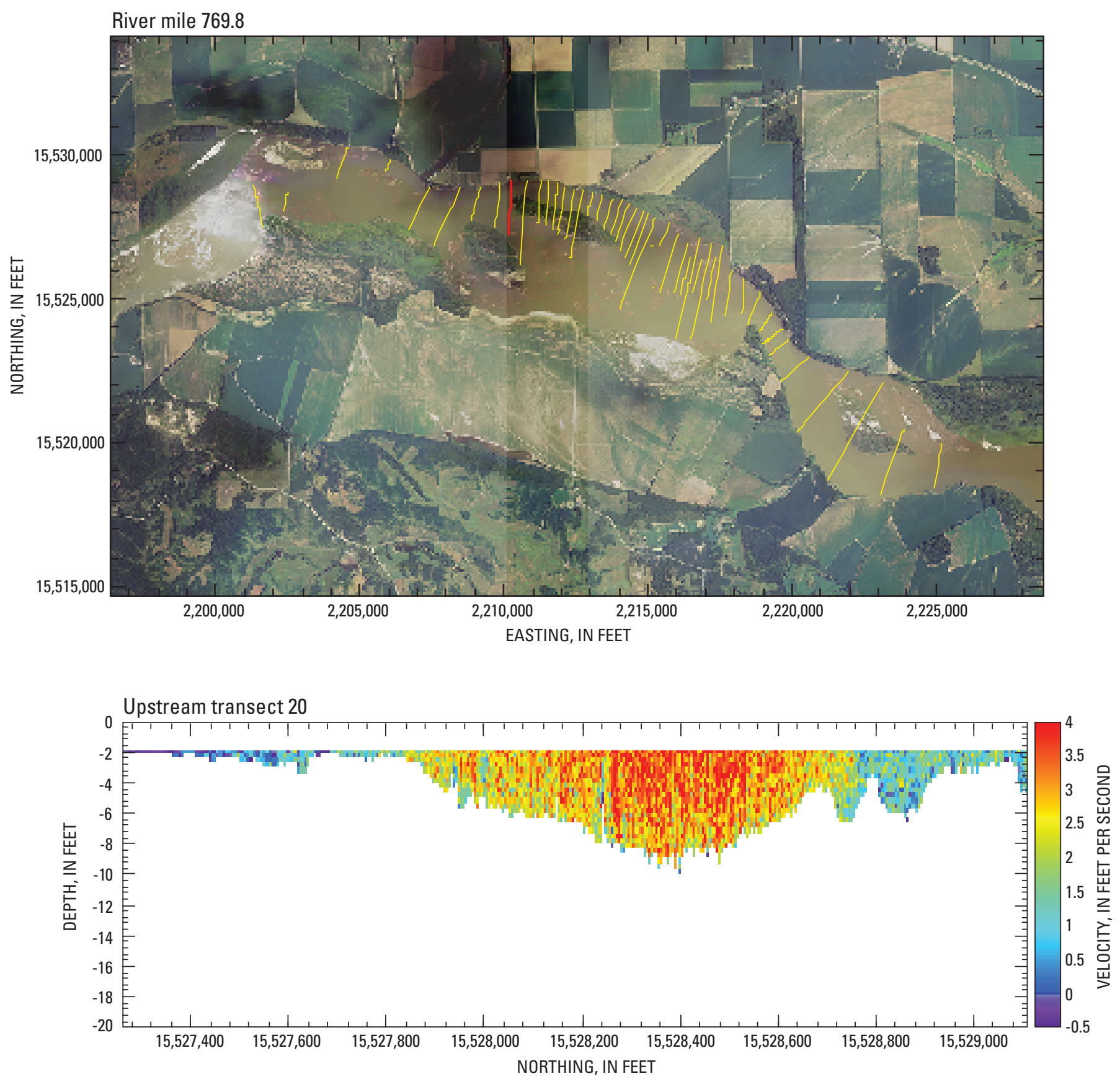

Base from Farm Service Agency digital orthophotography, 2003

Universal Transverse Mercator projection, Zone 14

Horizontal coordinates are referenced to the North American Datum of 1983 (NAD 83)

Figure B9. Location and velocity profile for upstream transect 20. 

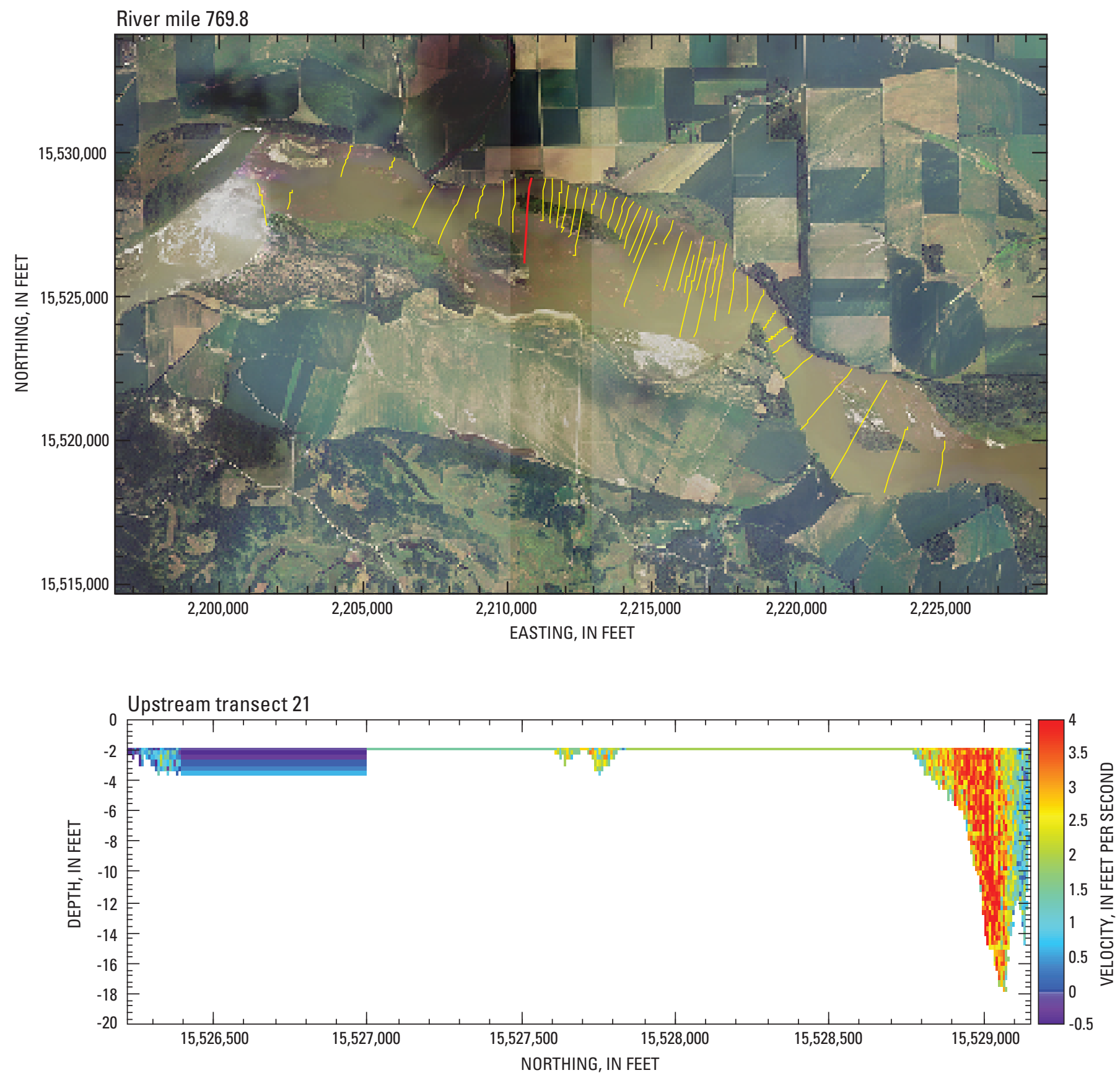

Base from Farm Service Agency digital orthophotography, 2003

Universal Transverse Mercator projection, Zone 14

Horizontal coordinates are referenced to the North American Datum of 1983 (NAD 83)

Figure B10. Location and velocity profile for upstream transect 21. 

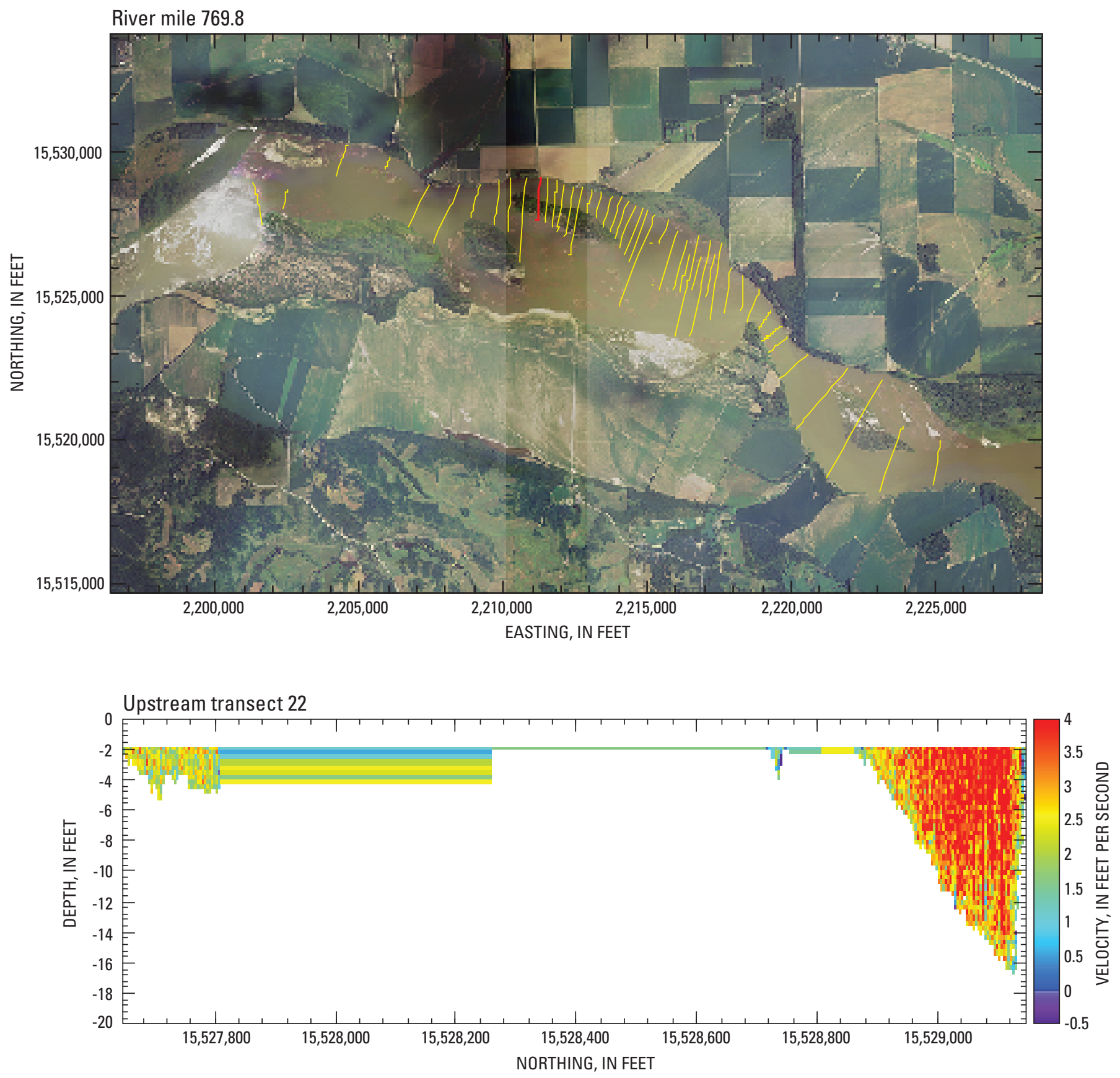

Base from Farm Service Agency digital orthophotography, 2003

Universal Transverse Mercator projection, Zone 14

Horizontal coordinates are referenced to the North American Datum of 1983 (NAD 83)

Figure B11. Location and velocity profile for upstream transect 22. 

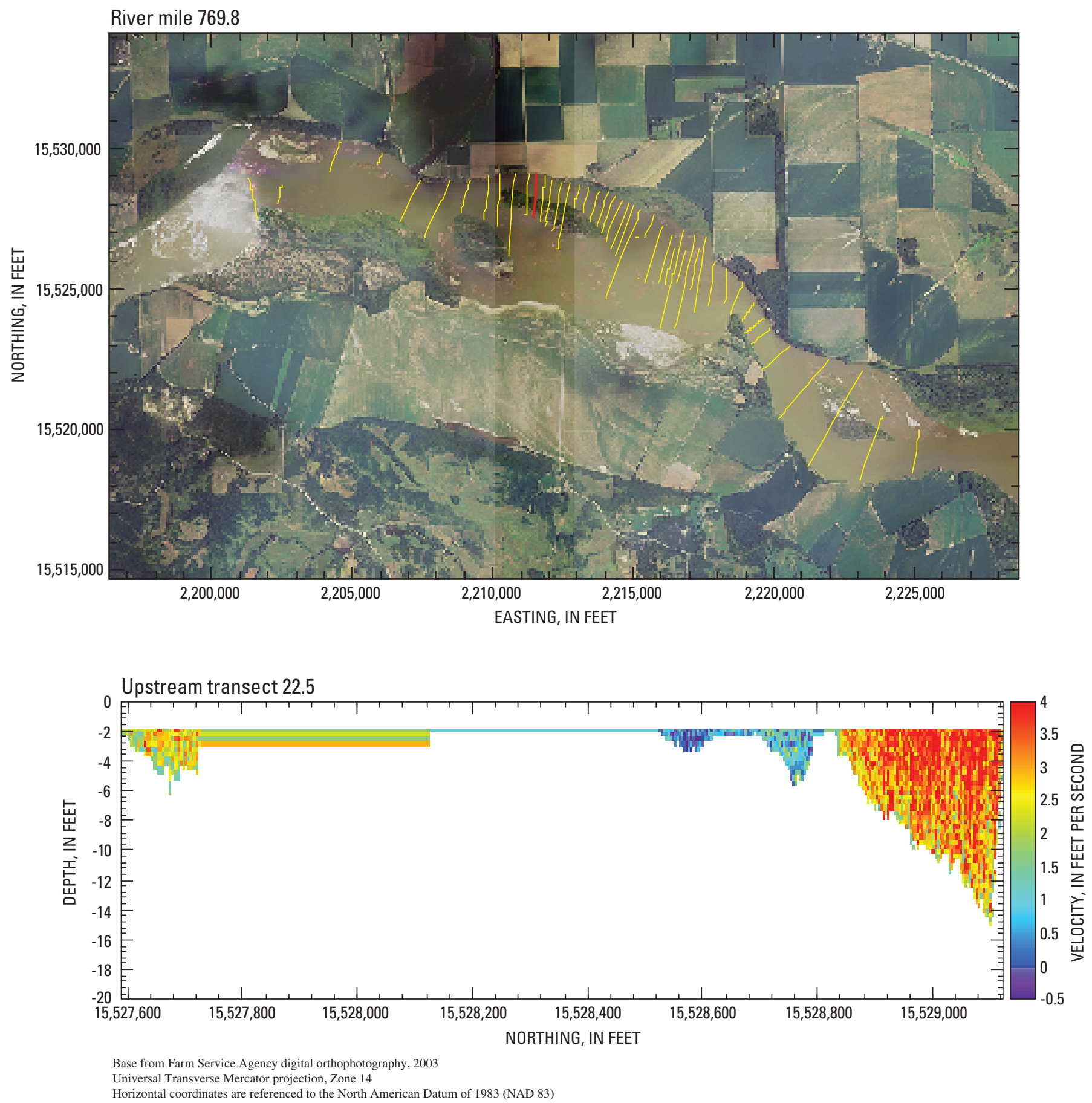

Figure B12. Location and velocity profile for upstream transect 22.5. 

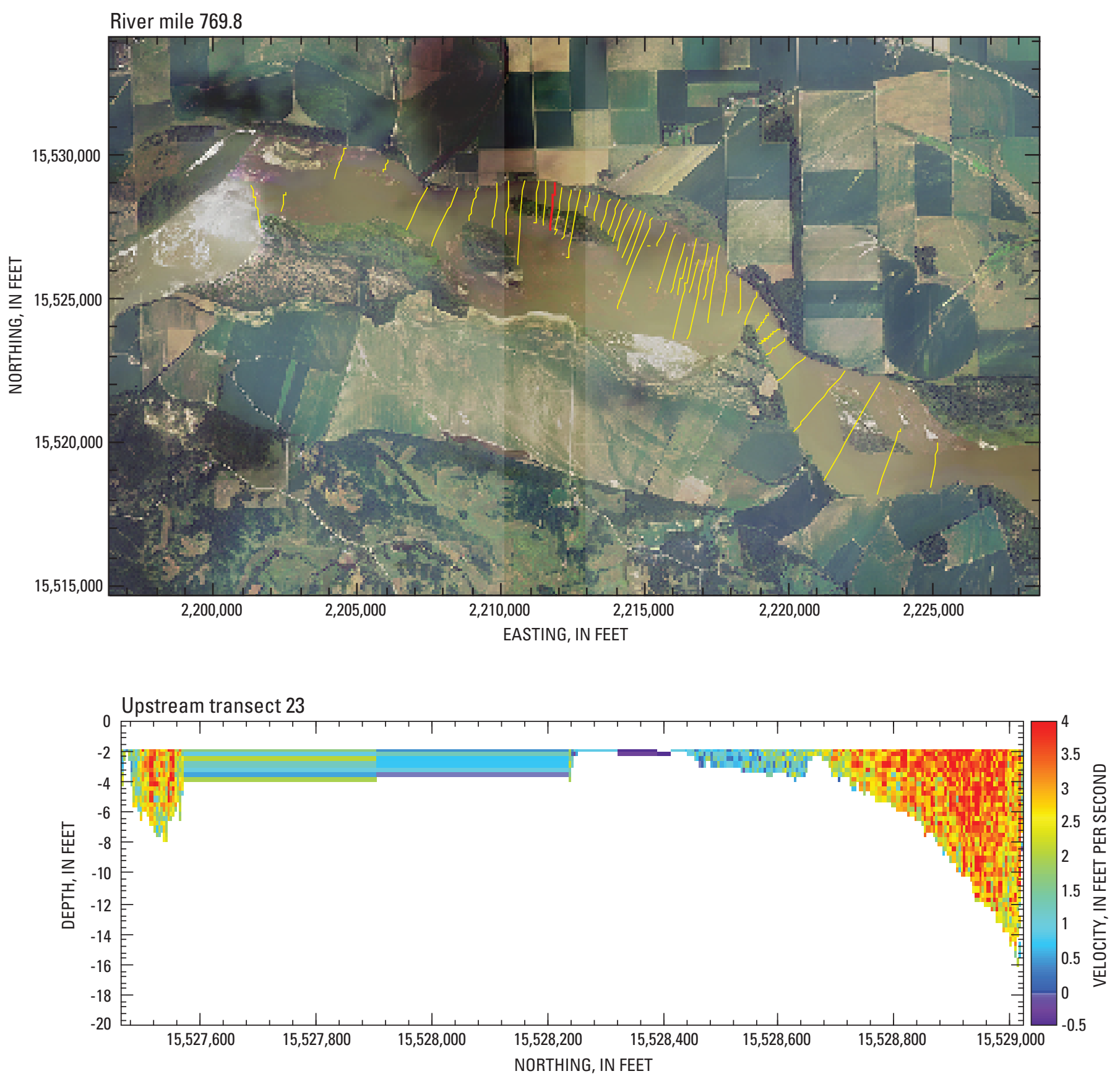

Base from Farm Service Agency digital orthophotography, 2003

Universal Transverse Mercator projection, Zone 14

Horizontal coordinates are referenced to the North American Datum of 1983 (NAD 83)

Figure B13. Location and velocity profile for upstream transect 23. 

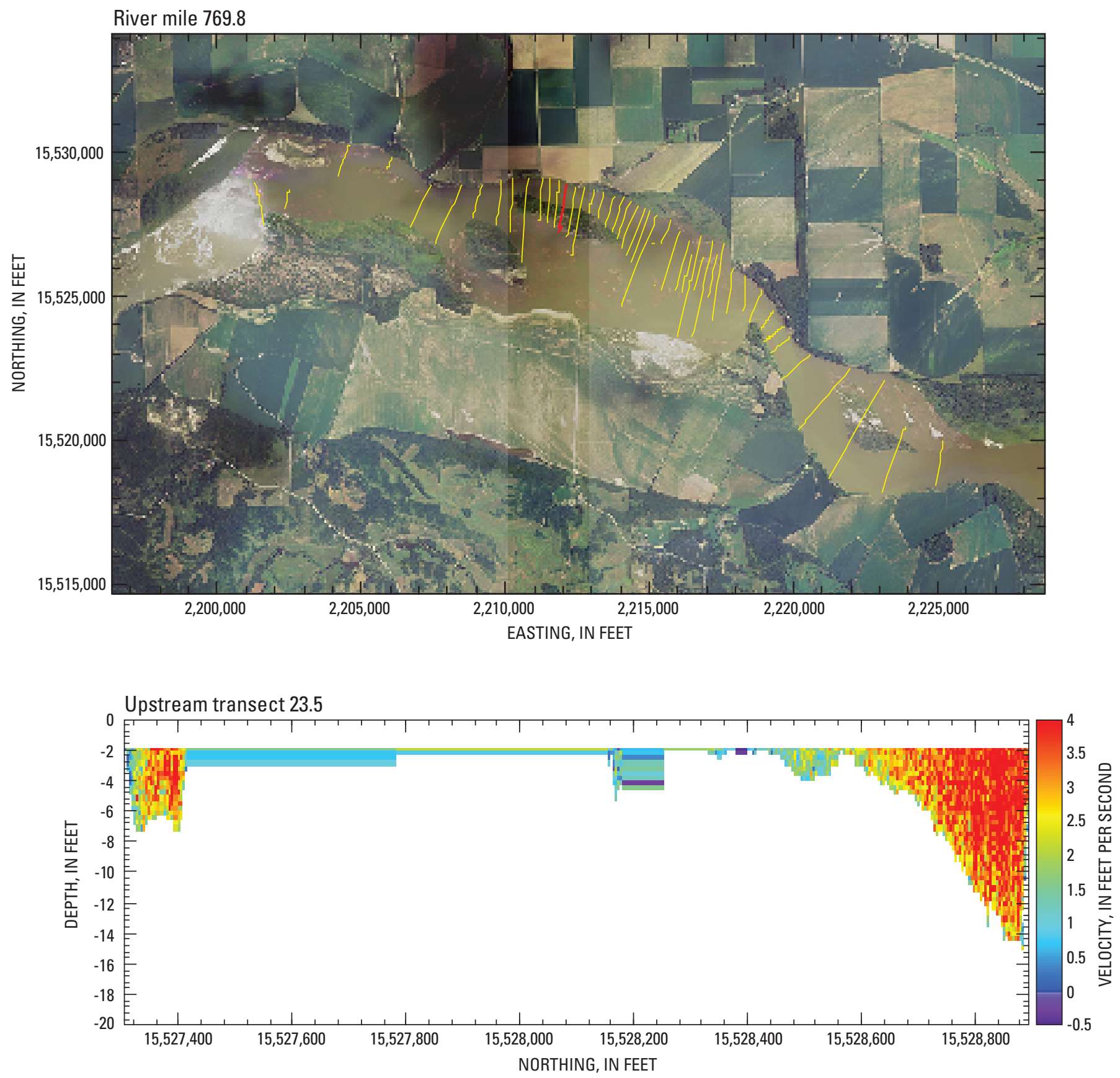

Base from Farm Service Agency digital orthophotography, 2003

Universal Transverse Mercator projection, Zone 14

Horizontal coordinates are referenced to the North American Datum of 1983 (NAD 83)

Figure B14. Location and velocity profile for upstream transect 23.5. 

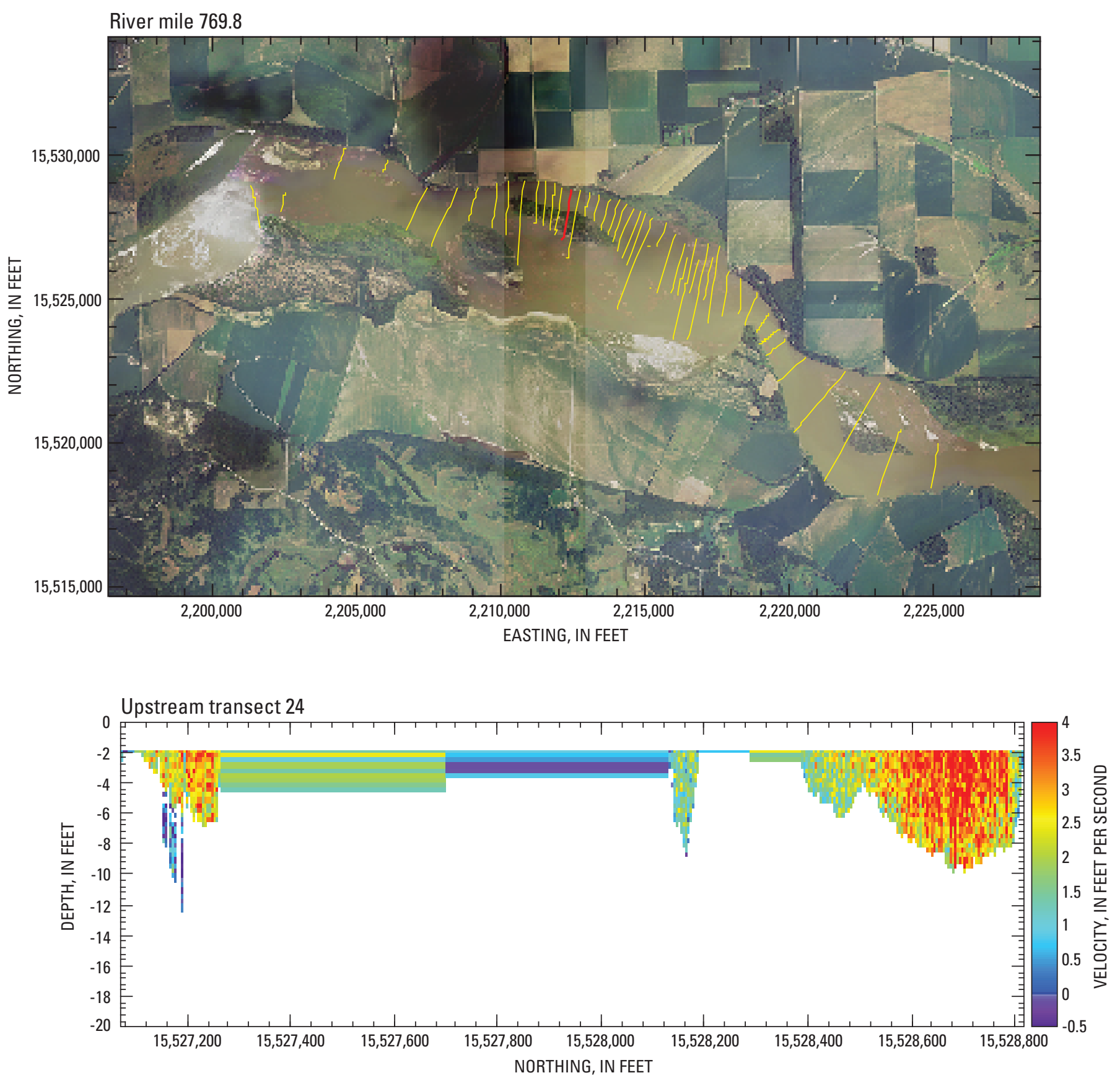

Base from Farm Service Agency digital orthophotography, 2003

Universal Transverse Mercator projection, Zone 14

Horizontal coordinates are referenced to the North American Datum of 1983 (NAD 83)

Figure B15. Location and velocity profile for upstream transect 24. 

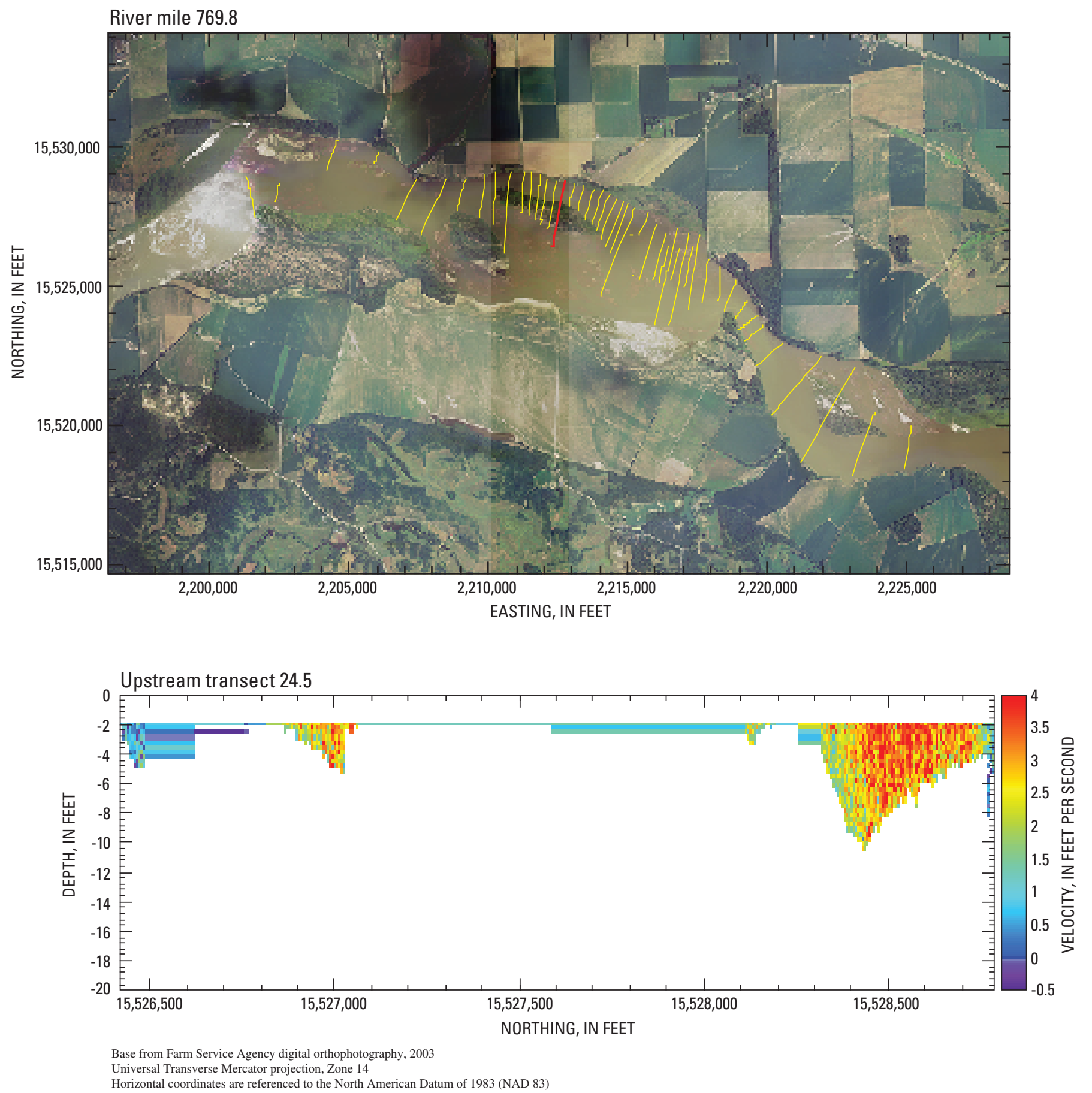

Figure B16. Location and velocity profile for upstream transect 24.5. 

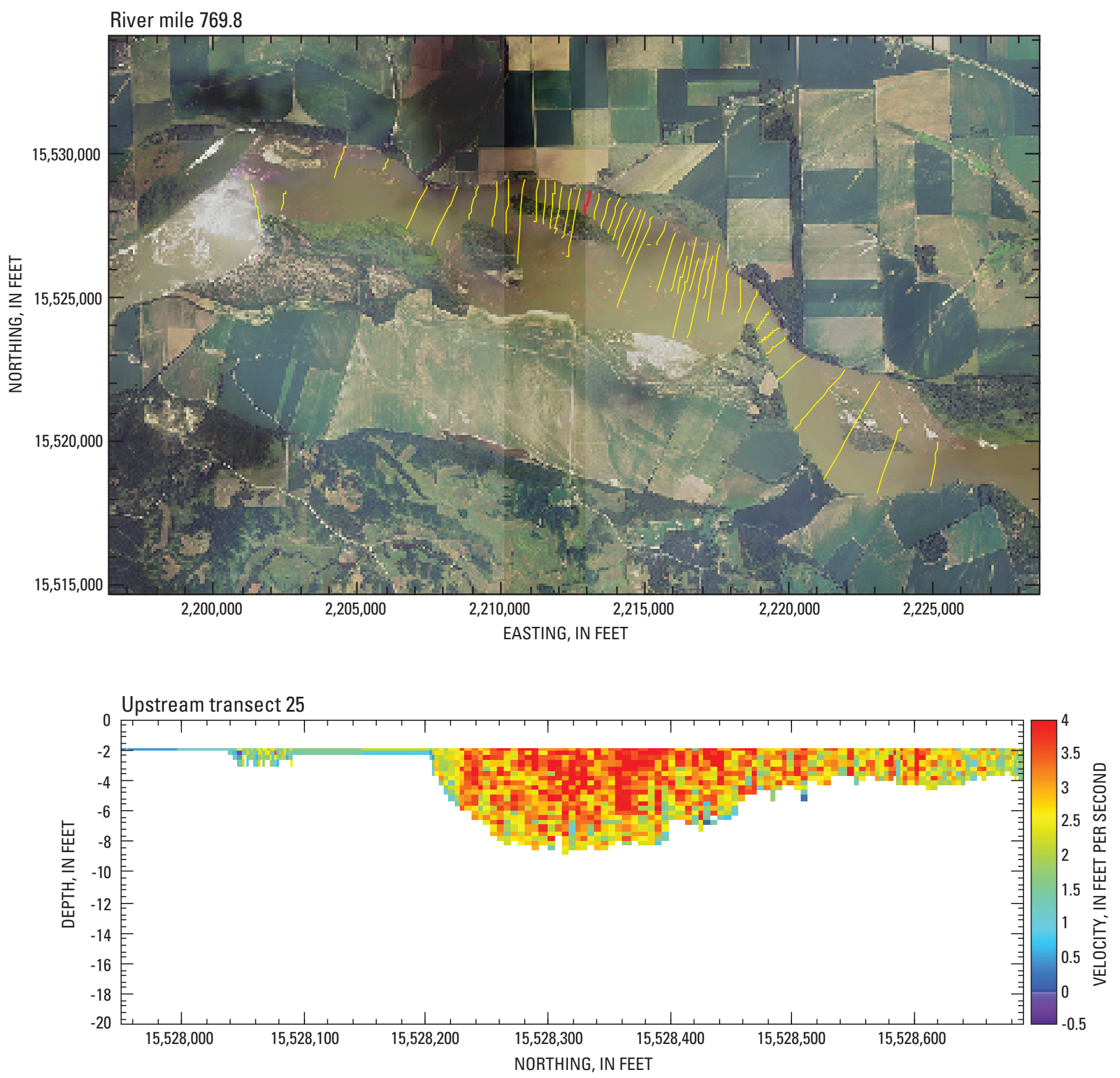

Base from Farm Service Agency digital orthophotography, 2003

Universal Transverse Mercator projection, Zone 14

Horizontal coordinates are referenced to the North American Datum of 1983 (NAD 83)

Figure B17. Location and velocity profile for upstream transect 25 . 

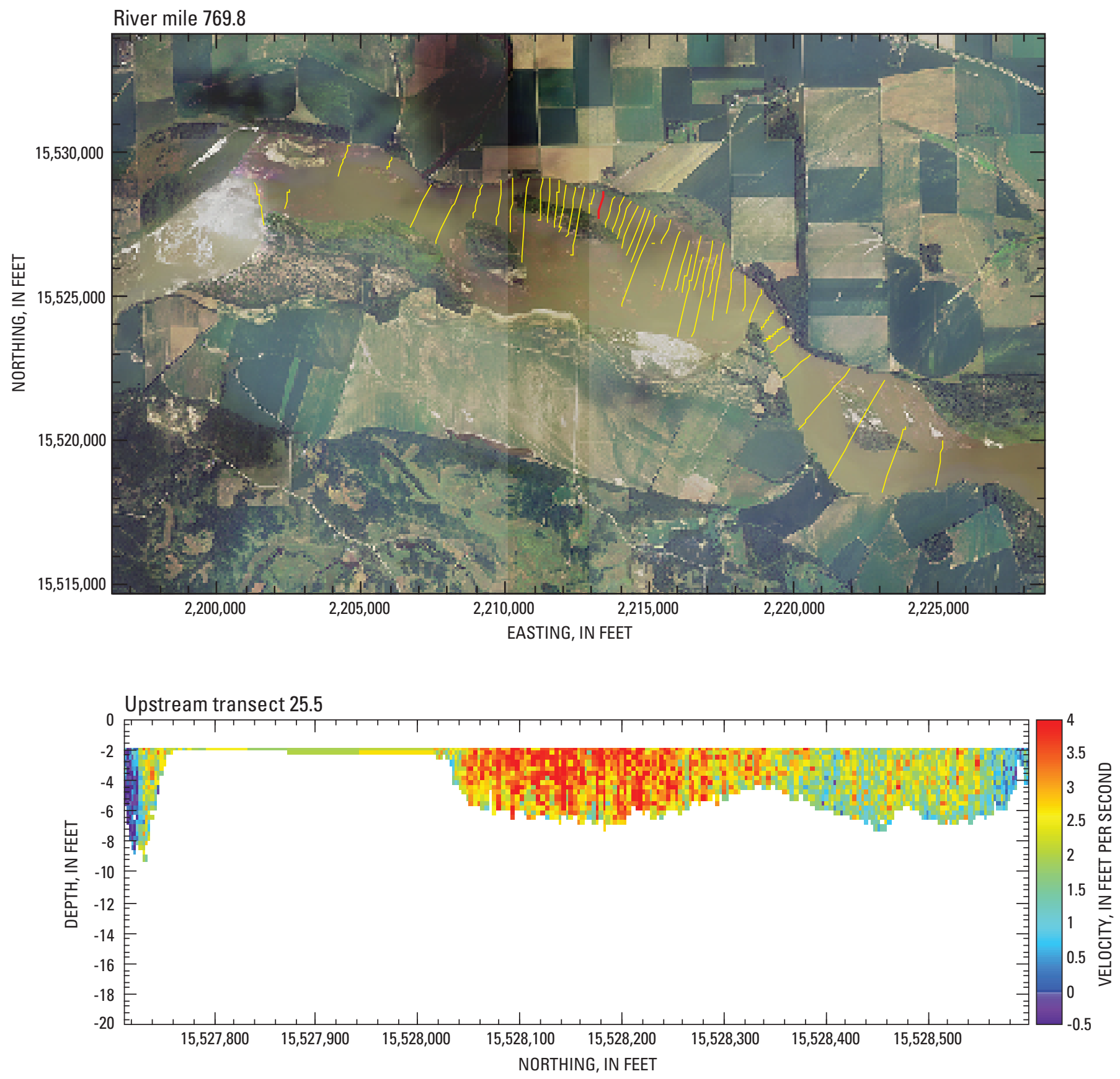

Base from Farm Service Agency digital orthophotography, 2003

Universal Transverse Mercator projection, Zone 14

Horizontal coordinates are referenced to the North American Datum of 1983 (NAD 83)

Figure B18. Location and velocity profile for upstream transect 25.5. 

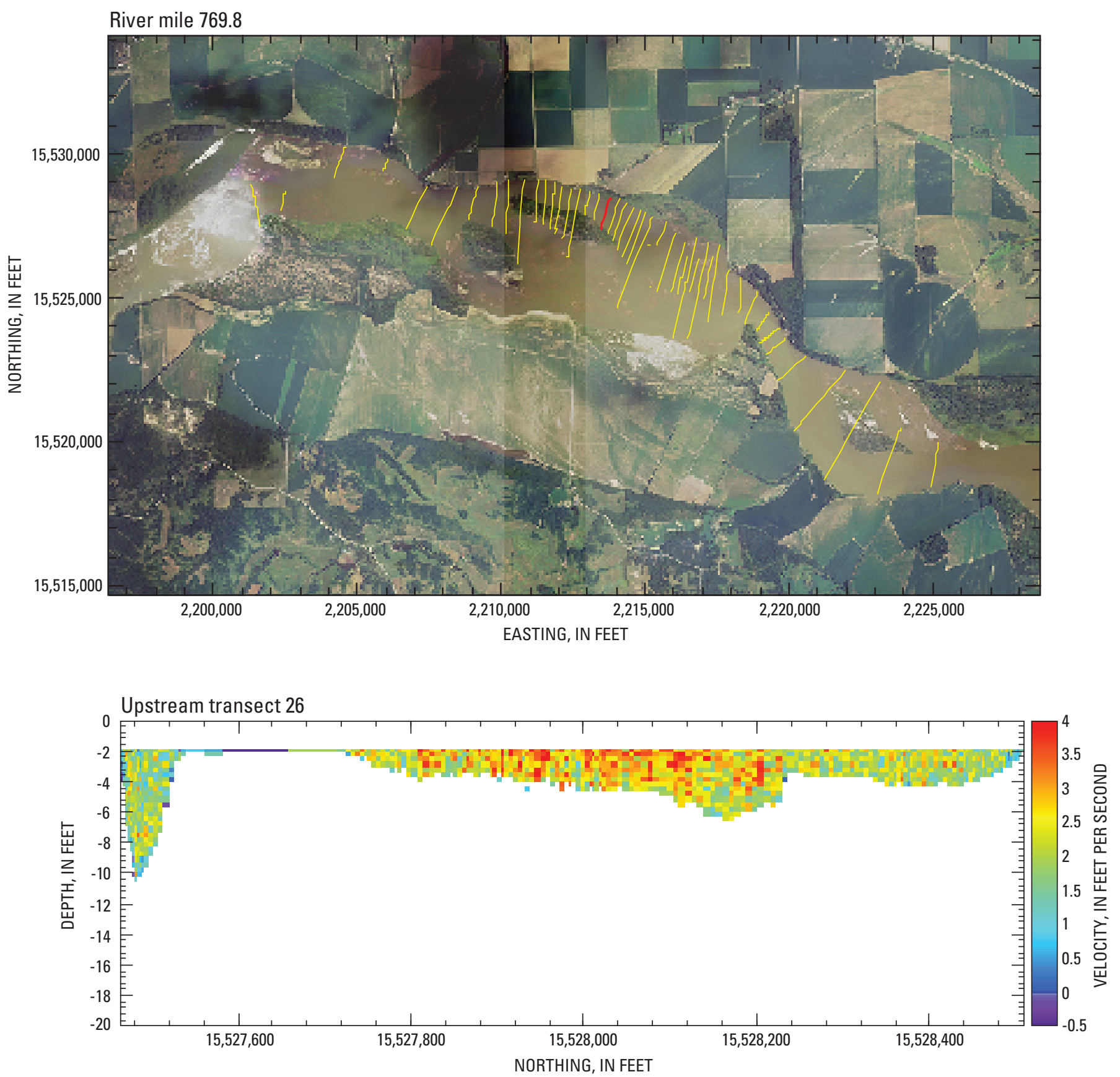

Base from Farm Service Agency digital orthophotography, 2003

Universal Transverse Mercator projection, Zone 14

Horizontal coordinates are referenced to the North American Datum of 1983 (NAD 83)

Figure B19. Location and velocity profile for upstream transect 26. 

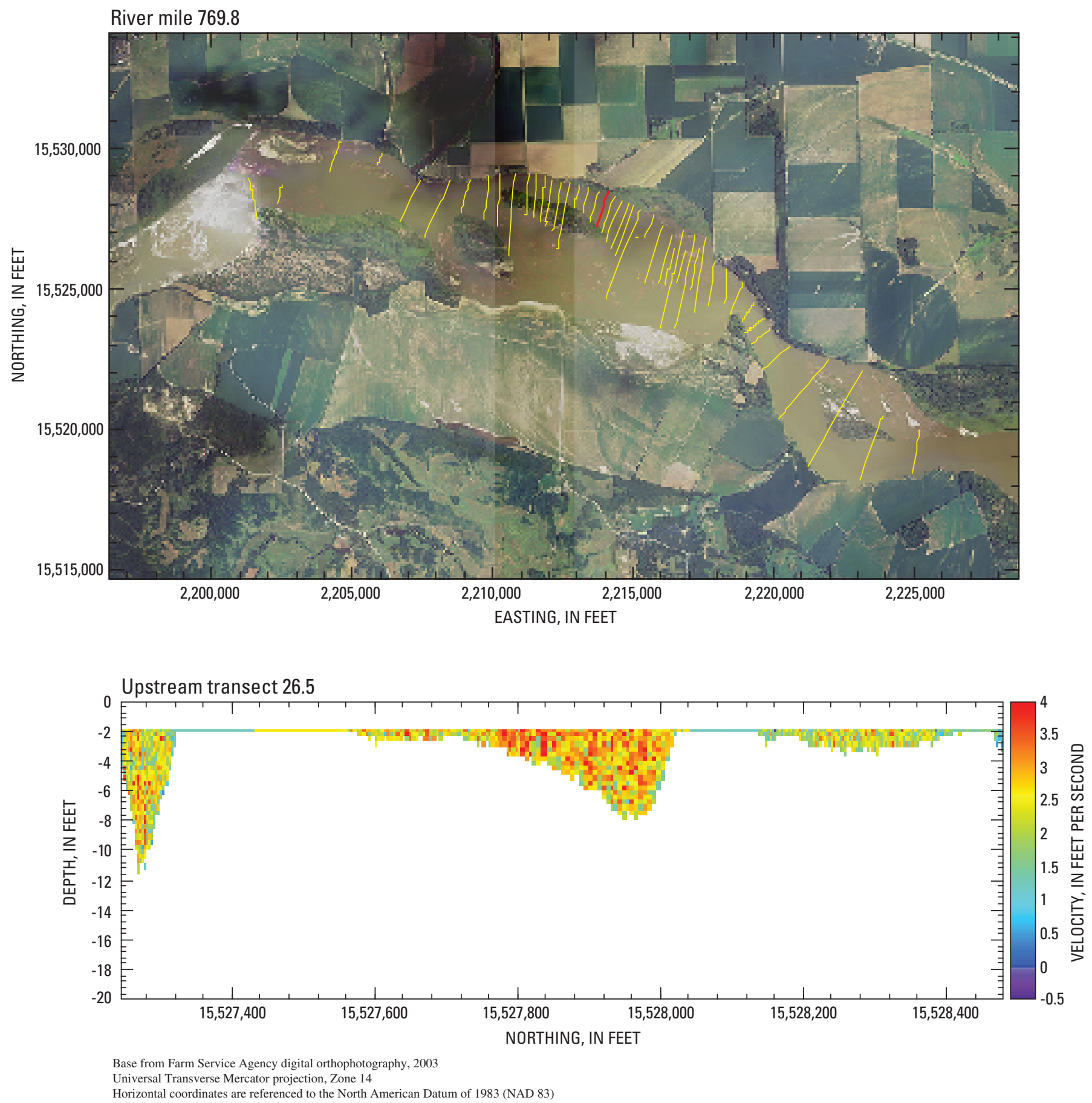

Figure B20. Location and velocity profile for upstream transect 26.5. 

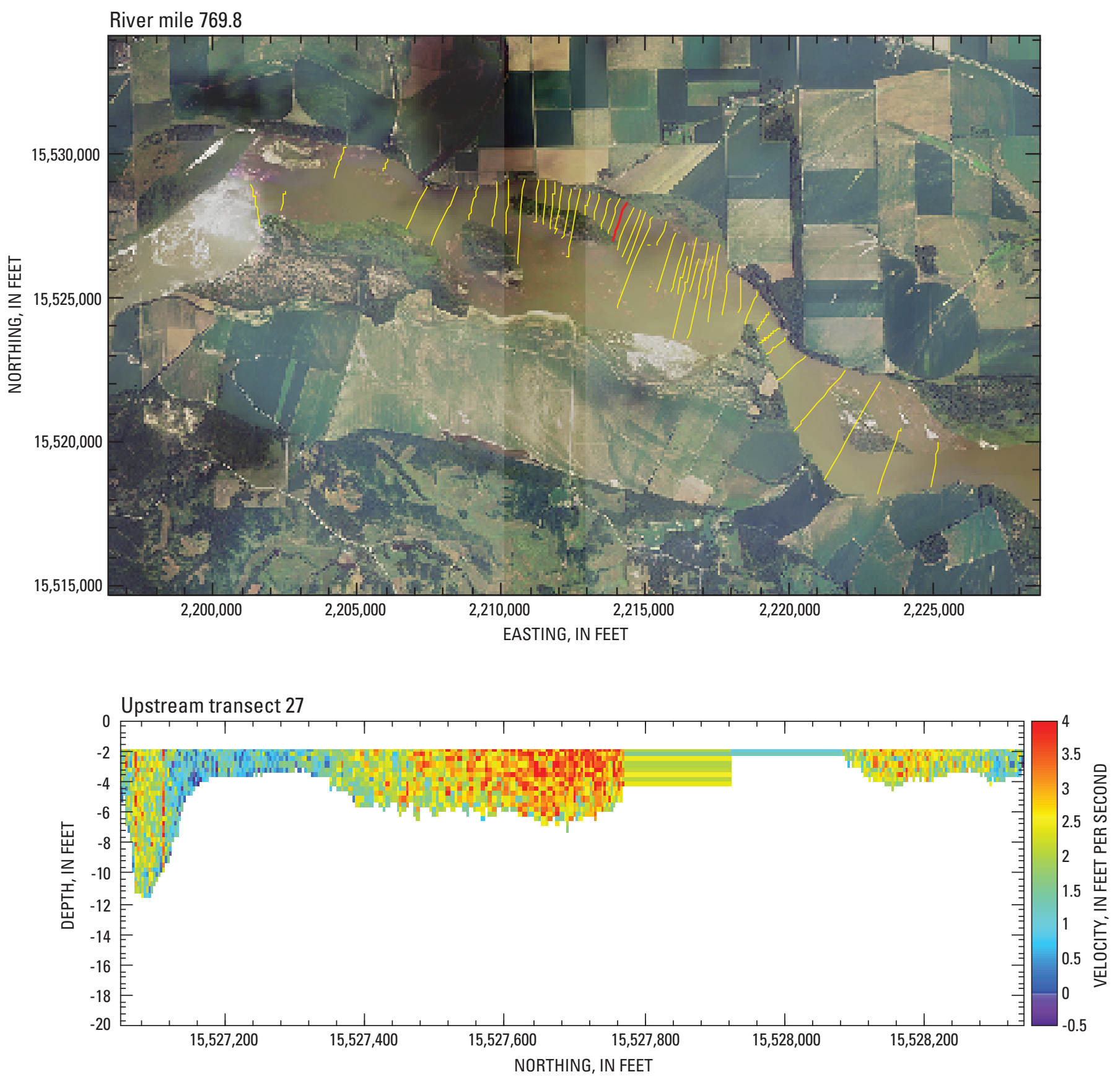

Base from Farm Service Agency digital orthophotography, 2003

Universal Transverse Mercator projection, Zone 14

Horizontal coordinates are referenced to the North American Datum of 1983 (NAD 83)

Figure B21. Location and velocity profile for upstream transect 27. 

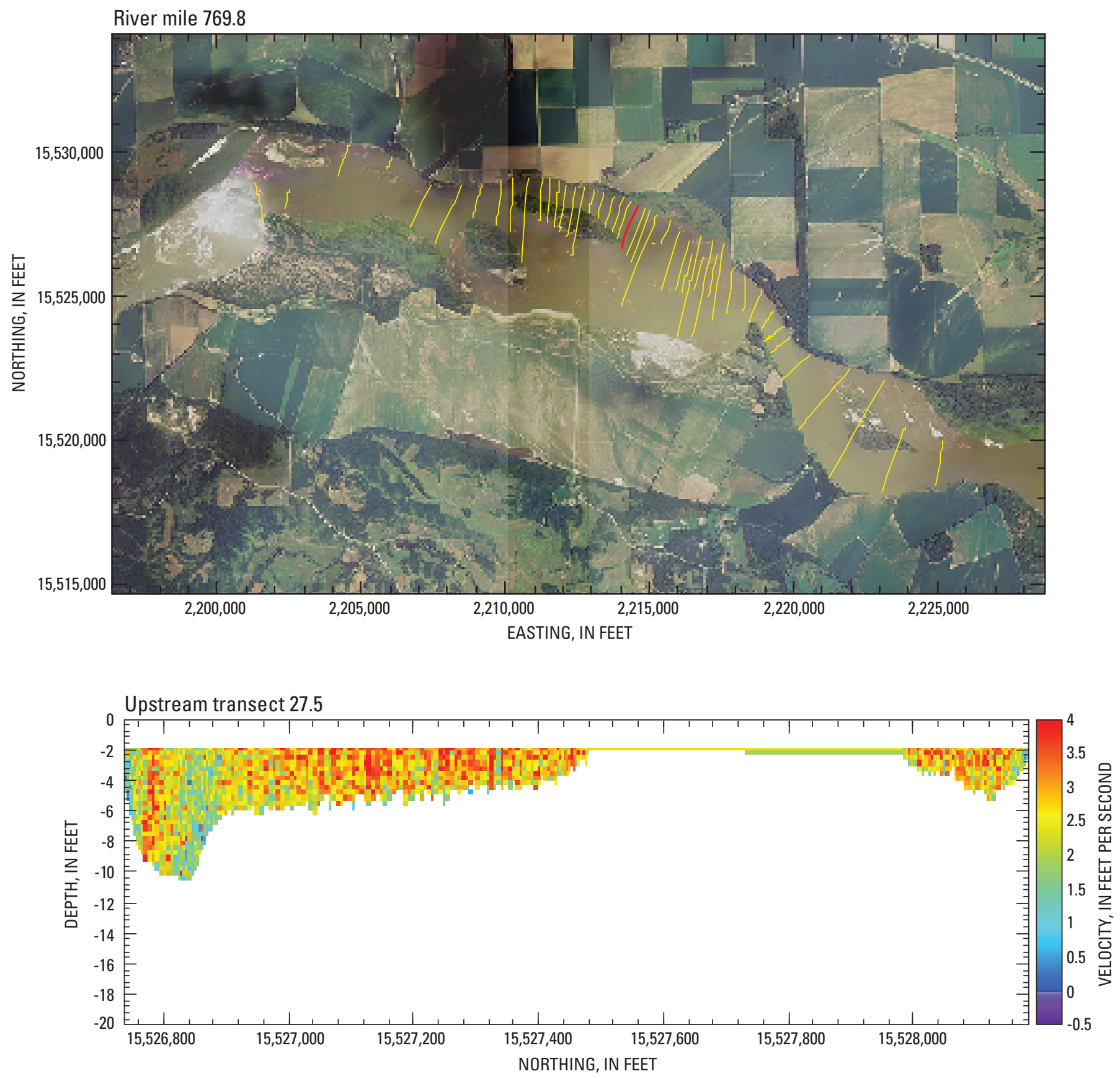

Base from Farm Service Agency digital orthophotography, 2003

Universal Transverse Mercator projection, Zone 14

Horizontal coordinates are referenced to the North American Datum of 1983 (NAD 83)

Figure B22. Location and velocity profile for upstream transect 27.5. 

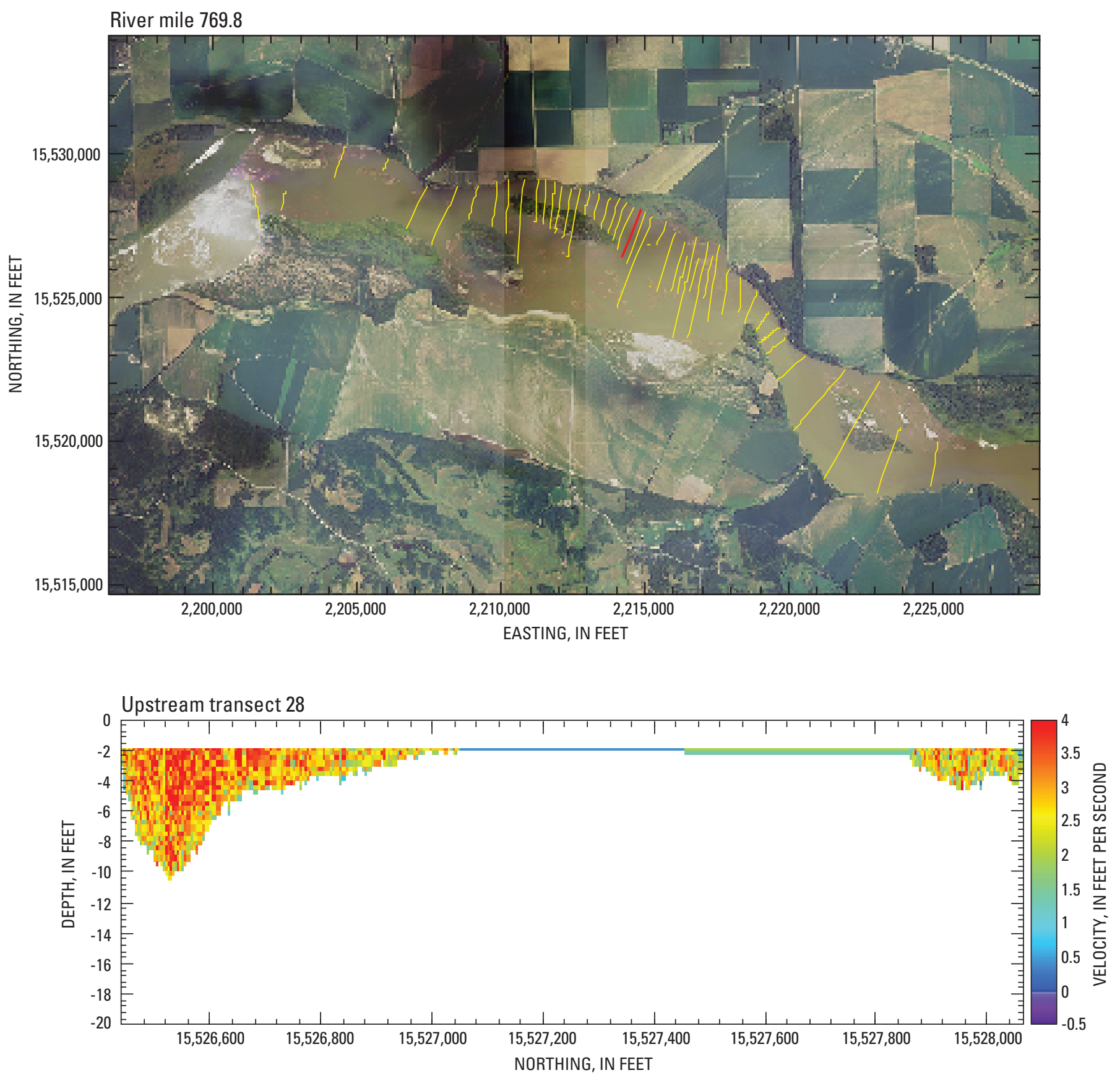

Base from Farm Service Agency digital orthophotography, 2003

Universal Transverse Mercator projection, Zone 14

Horizontal coordinates are referenced to the North American Datum of 1983 (NAD 83)

Figure B23. Location and velocity profile for upstream transect 28. 

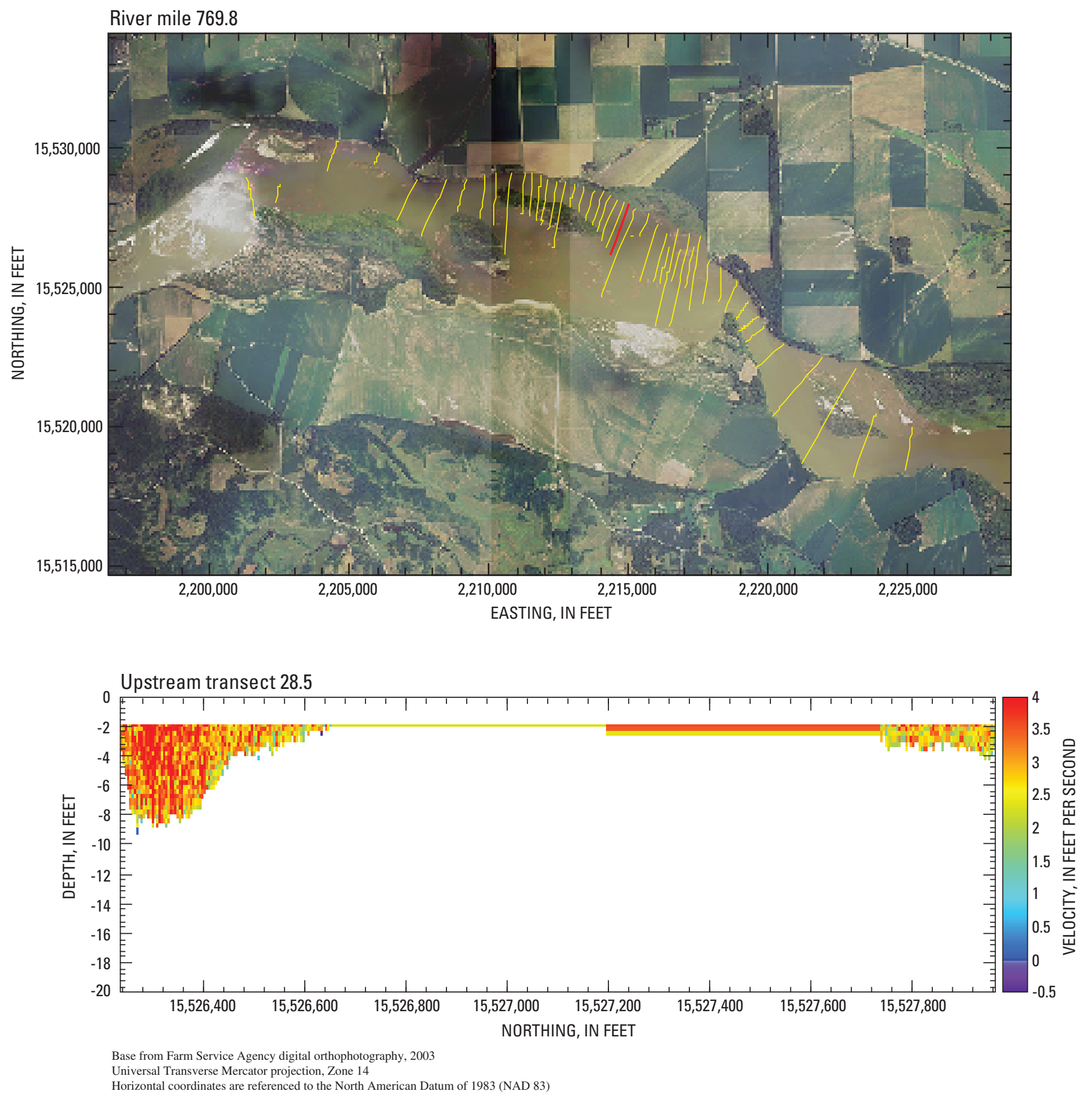

Figure B24. Location and velocity profile for upstream transect 28.5 . 

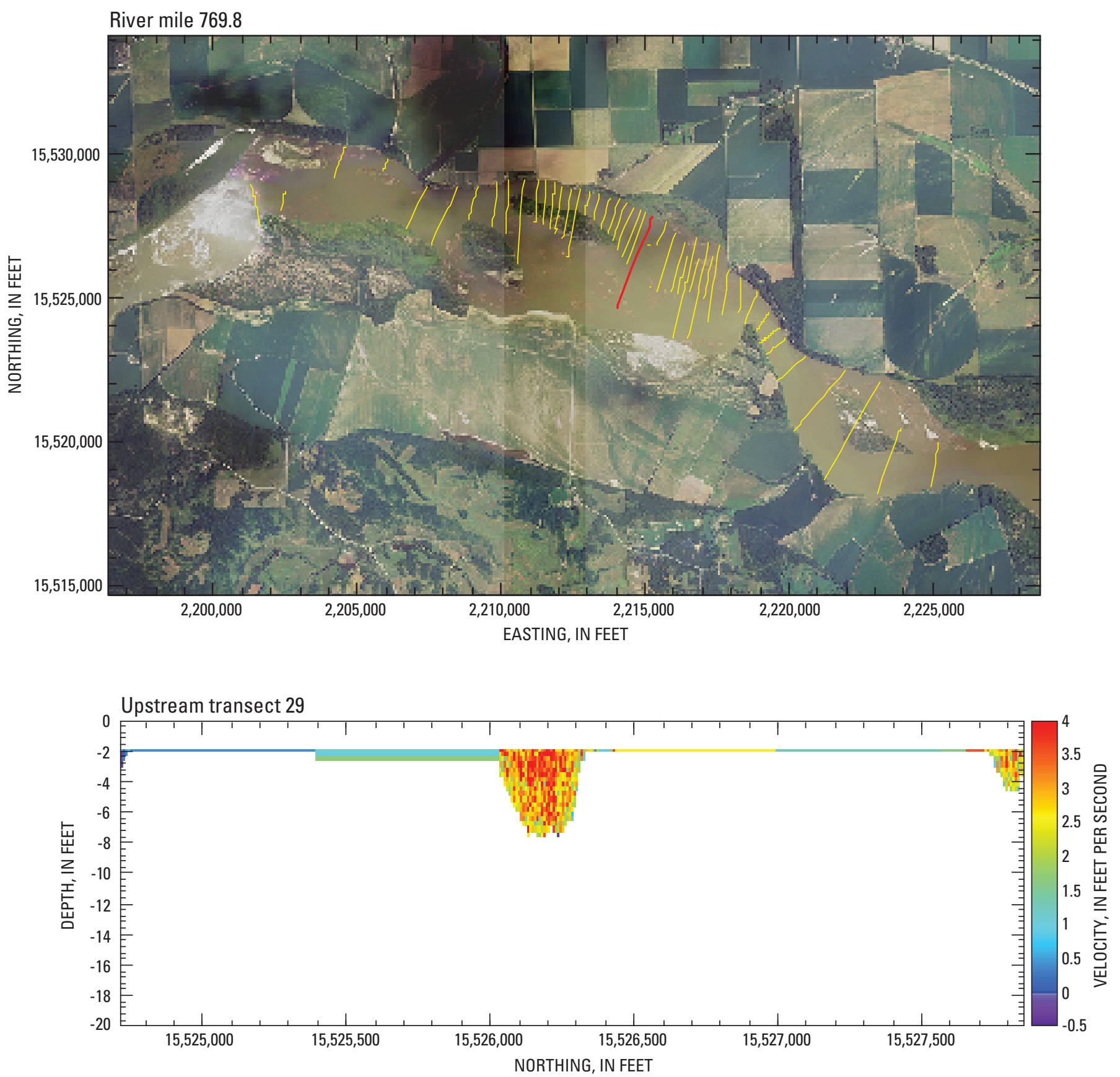

Base from Farm Service Agency digital orthophotography, 2003

Universal Transverse Mercator projection, Zone 14

Horizontal coordinates are referenced to the North American Datum of 1983 (NAD 83)

Figure B25. Location and velocity profile for upstream transect 29. 

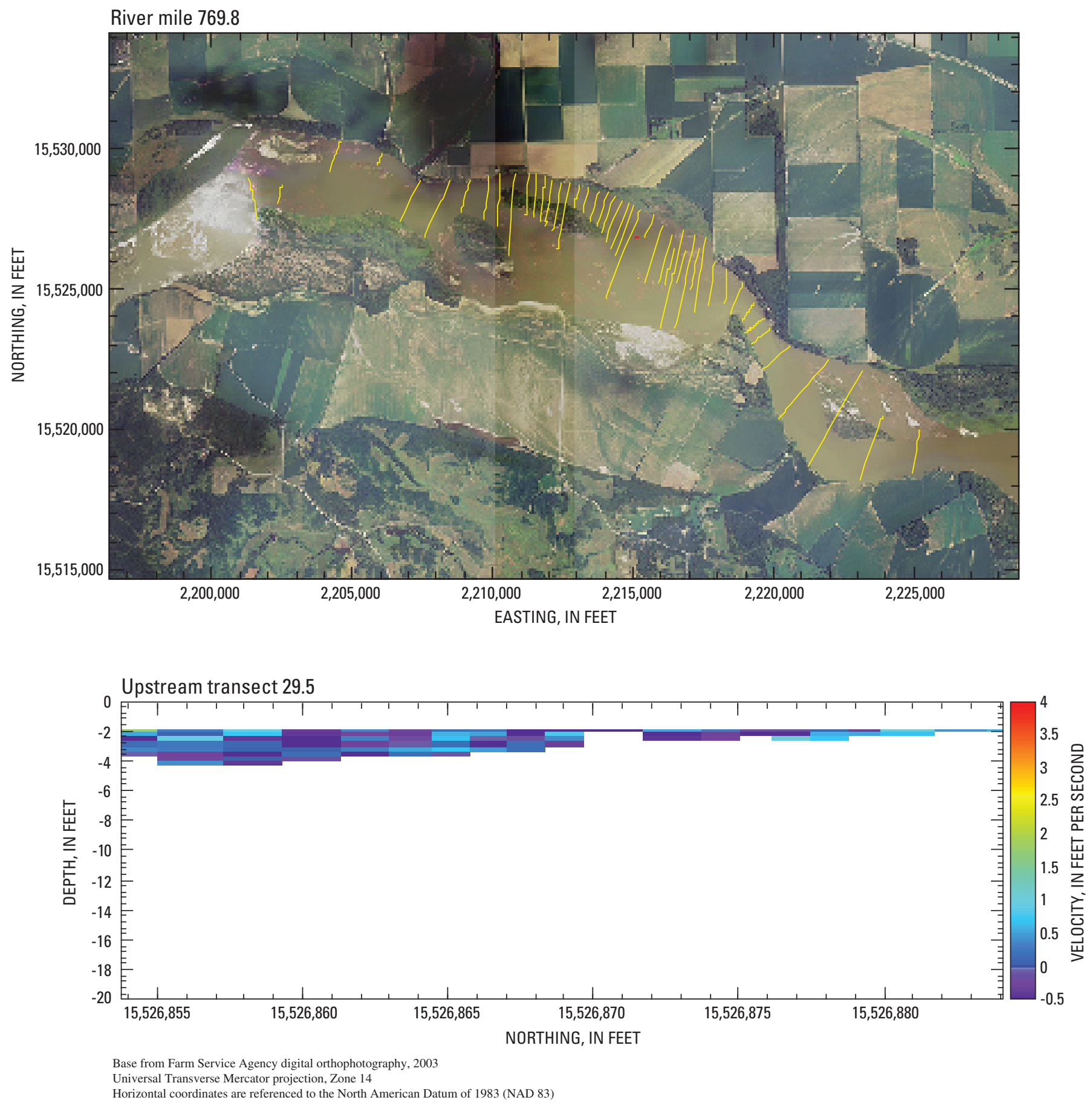

Figure B26. Location and velocity profile for upstream transect 29.5. 

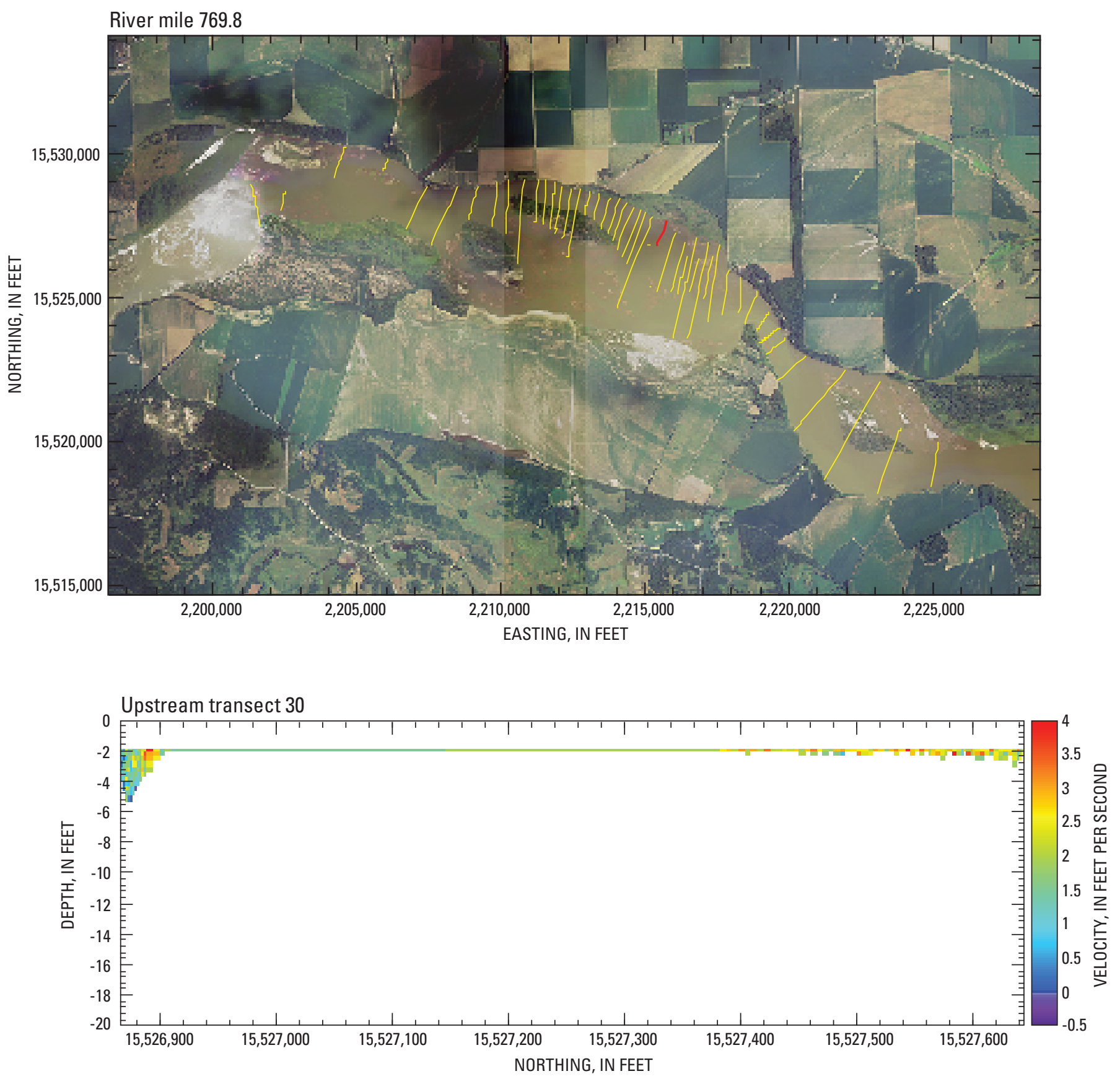

Base from Farm Service Agency digital orthophotography, 2003

Universal Transverse Mercator projection, Zone 14

Horizontal coordinates are referenced to the North American Datum of 1983 (NAD 83)

Figure B27. Location and velocity profile for upstream transect 30 . 

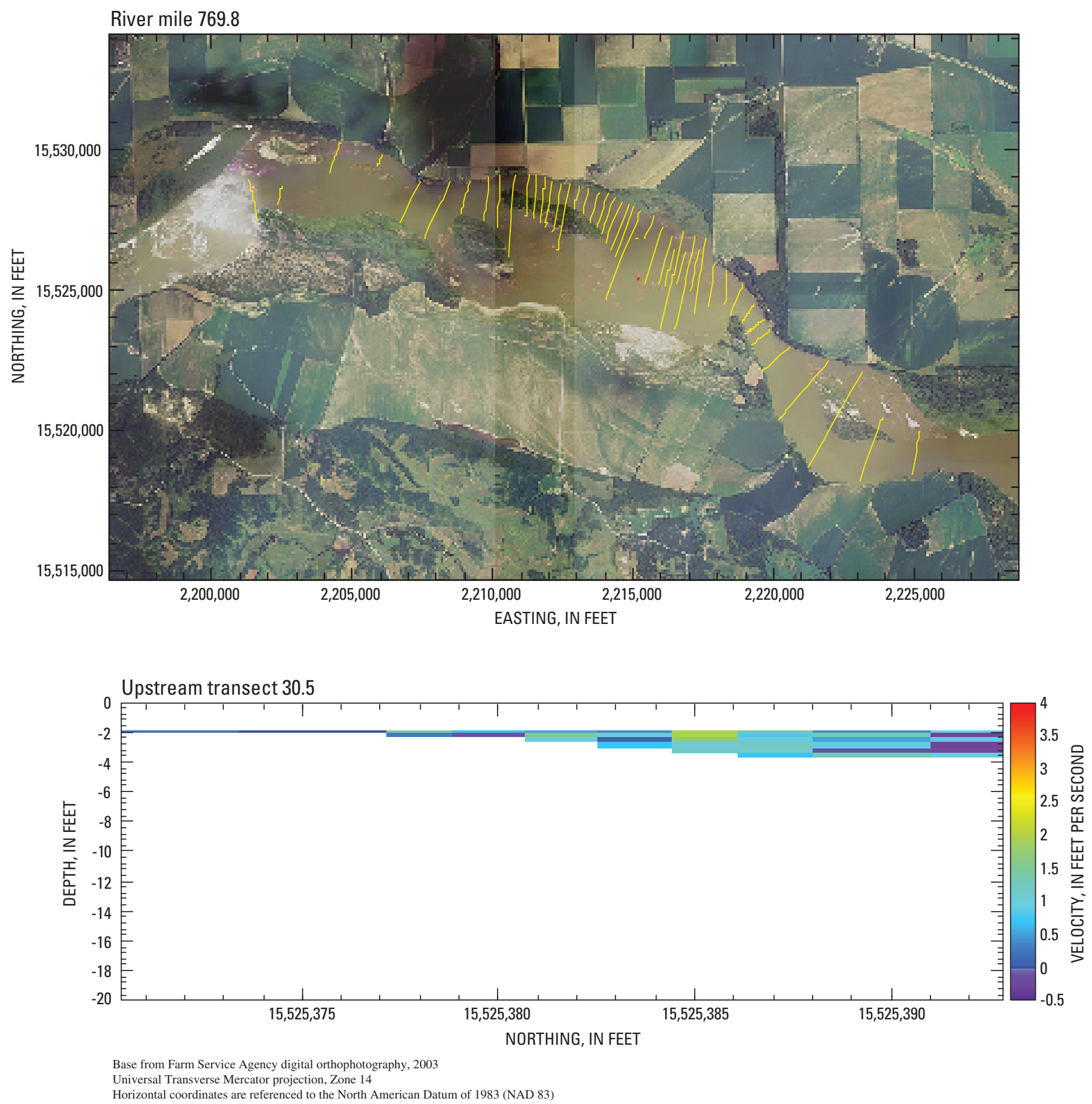

Figure B28. Location and velocity profile for upstream transect 30.5 . 

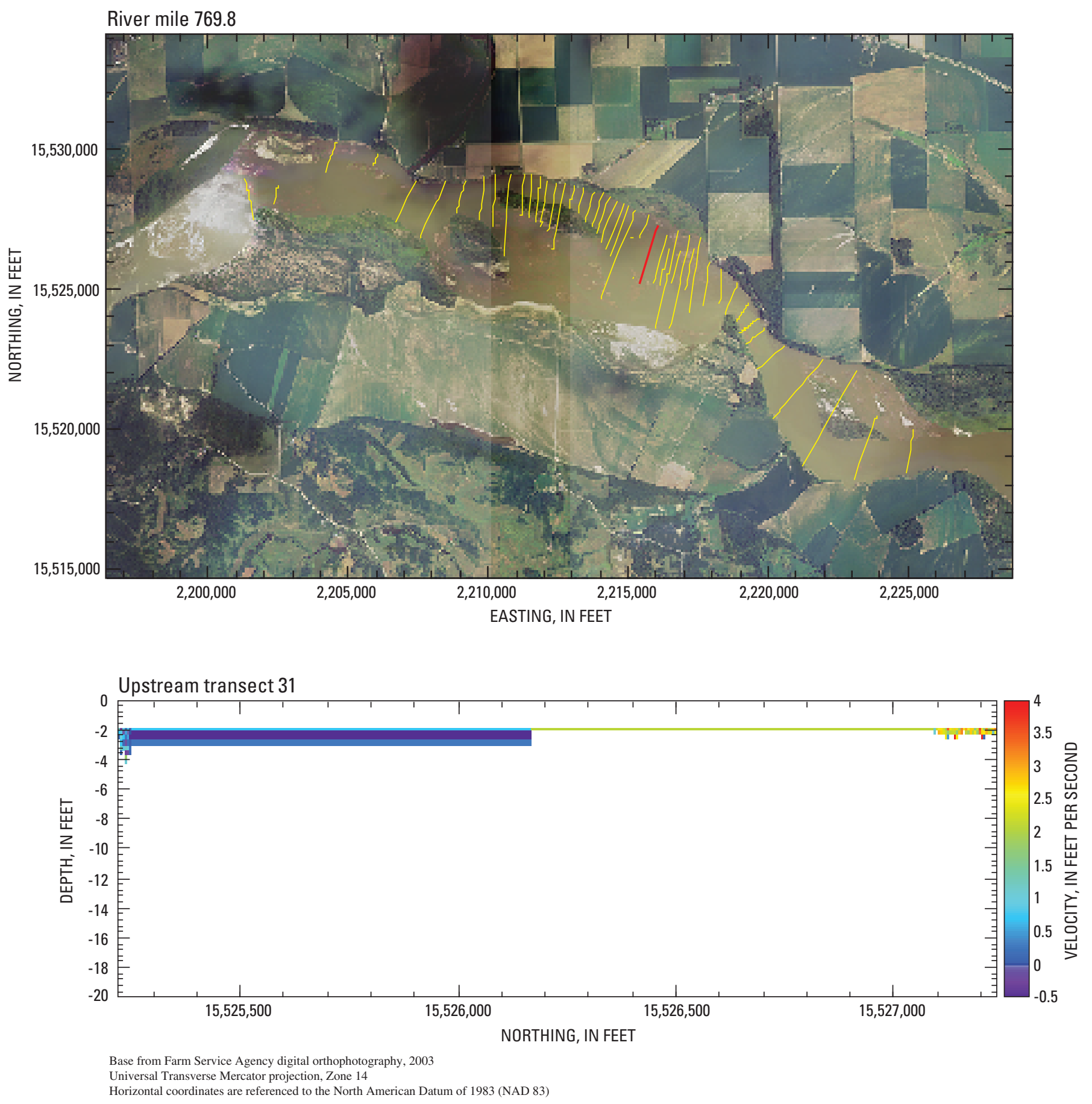

Figure B29. Location and velocity profile for upstream transect 31 . 

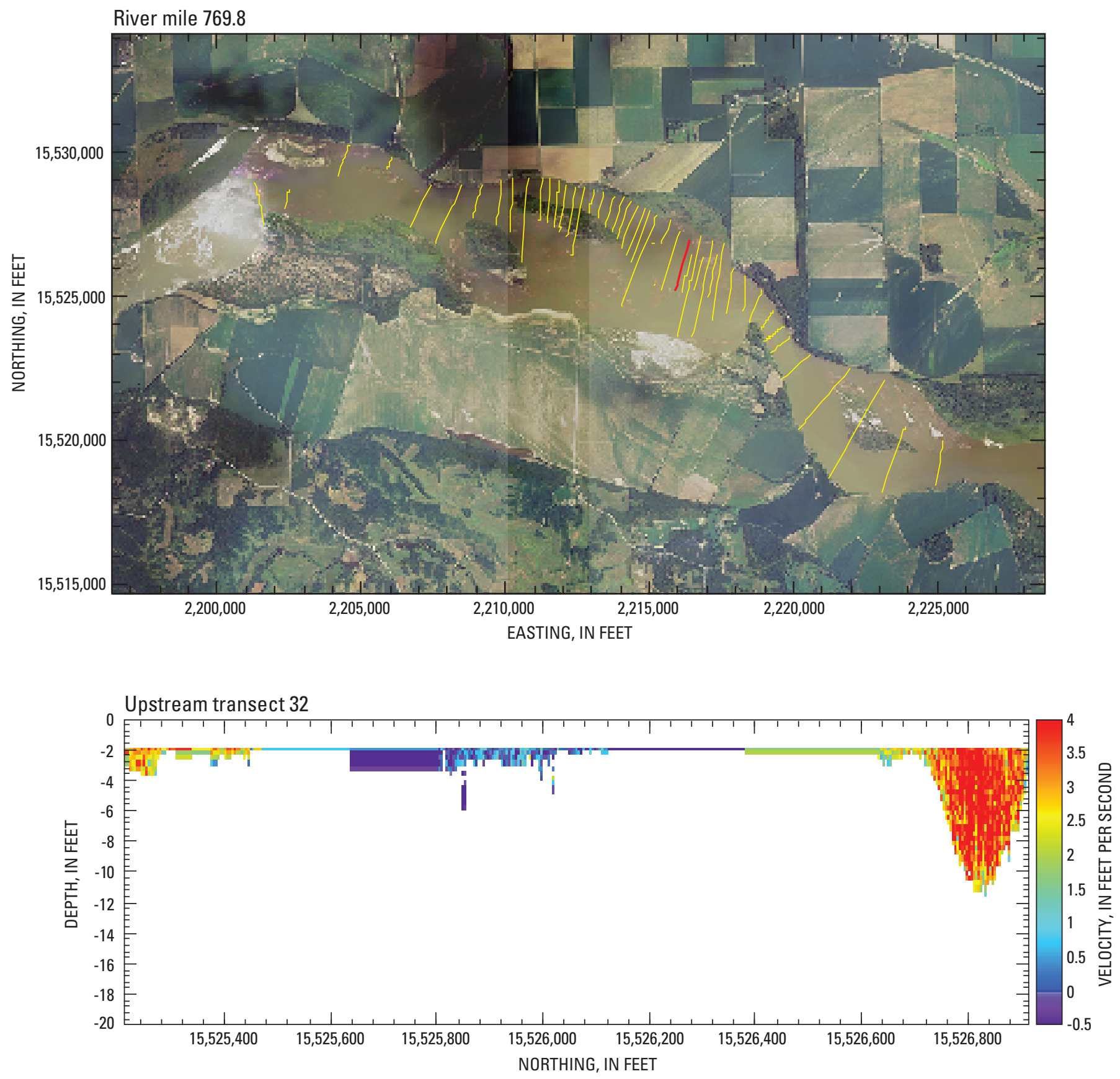

Base from Farm Service Agency digital orthophotography, 2003

Universal Transverse Mercator projection, Zone 14

Horizontal coordinates are referenced to the North American Datum of 1983 (NAD 83)

Figure B30. Location and velocity profile for upstream transect 32. 

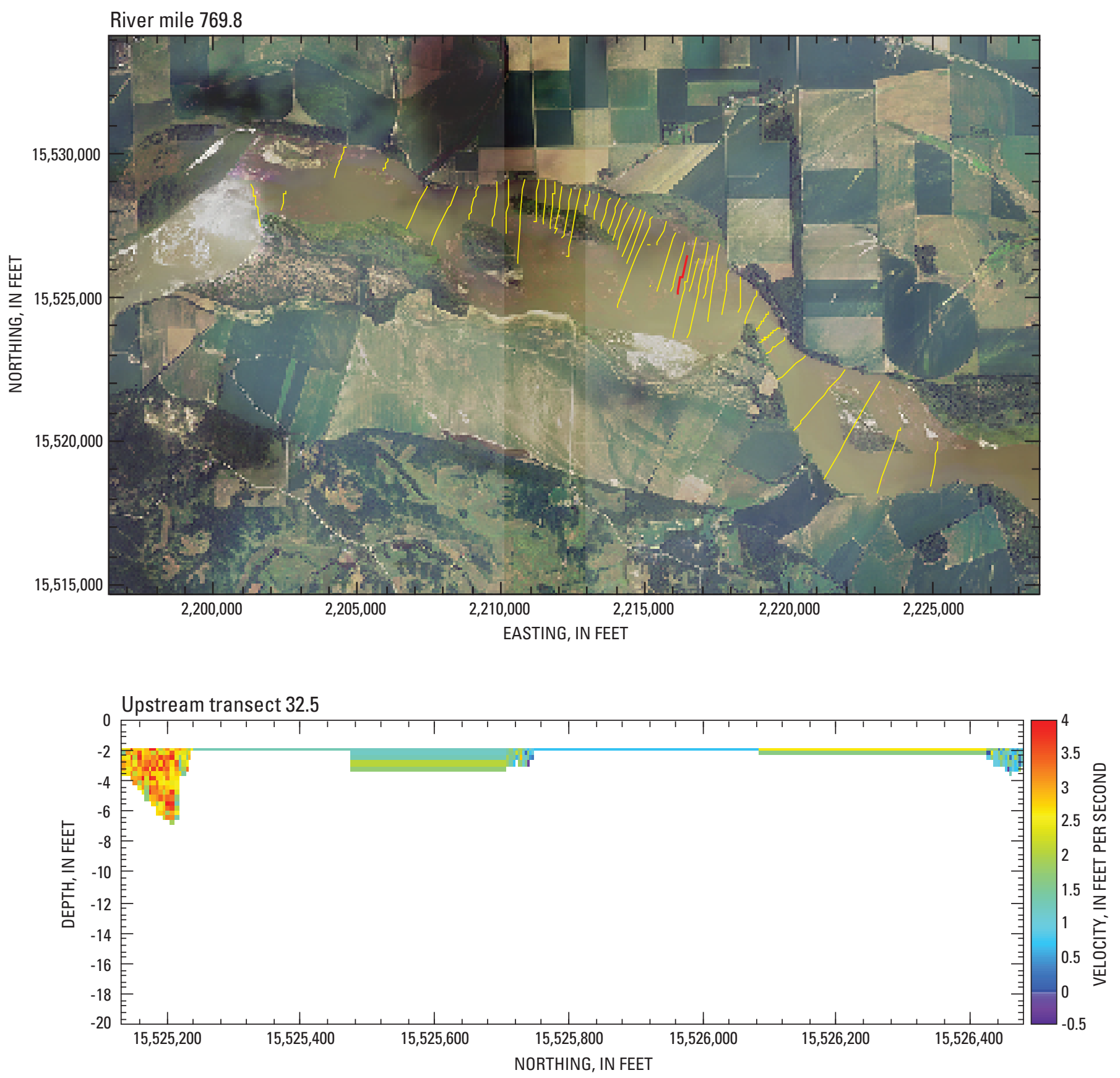

Base from Farm Service Agency digital orthophotography, 2003

Universal Transverse Mercator projection, Zone 14

Horizontal coordinates are referenced to the North American Datum of 1983 (NAD 83)

Figure B31. Location and velocity profile for upstream transect 32.5 . 

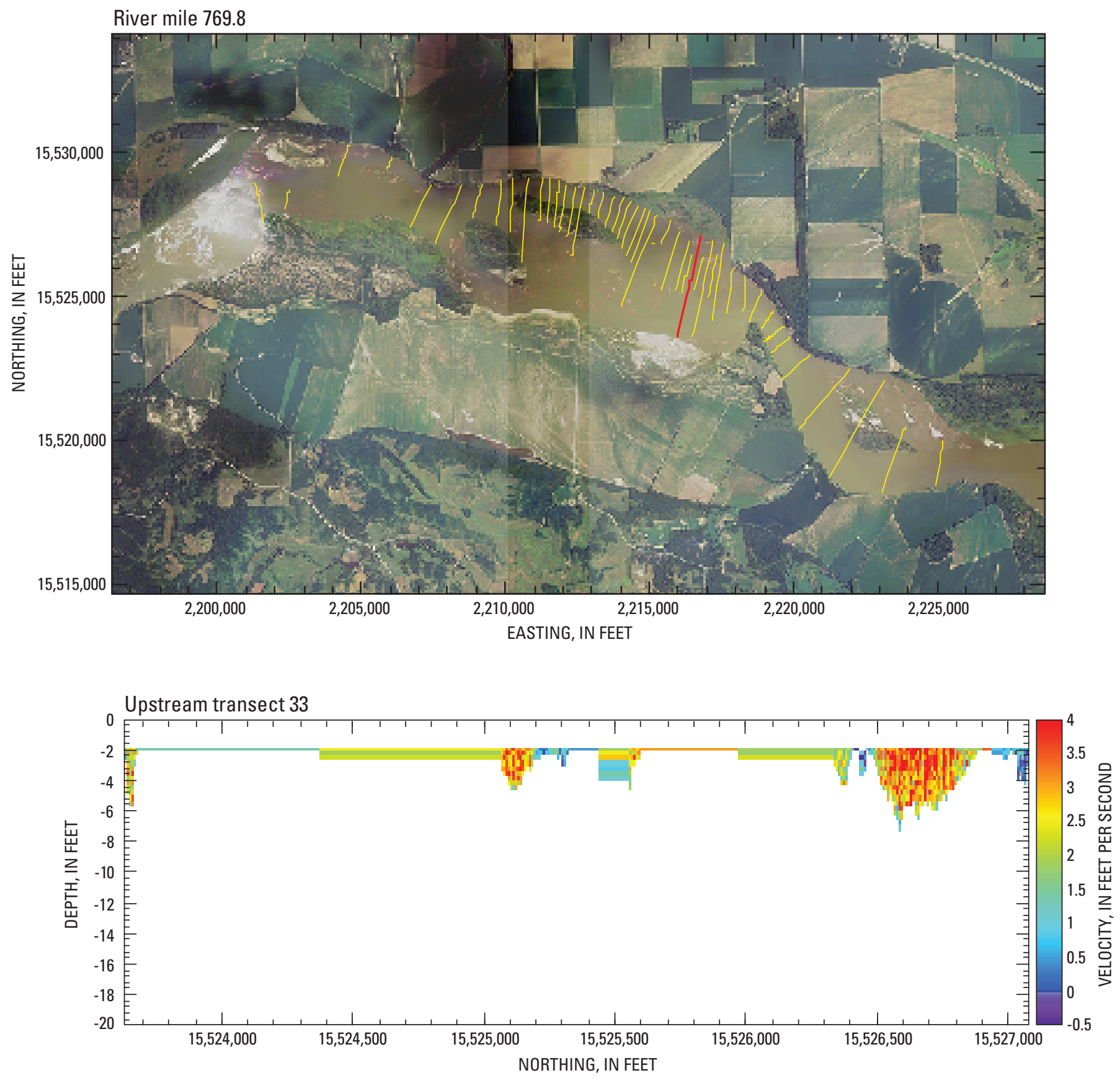

Base from Farm Service Agency digital orthophotography, 2003

Universal Transverse Mercator projection, Zone 14

Horizontal coordinates are referenced to the North American Datum of 1983 (NAD 83)

Figure B32. Location and velocity profile for upstream transect 33. 

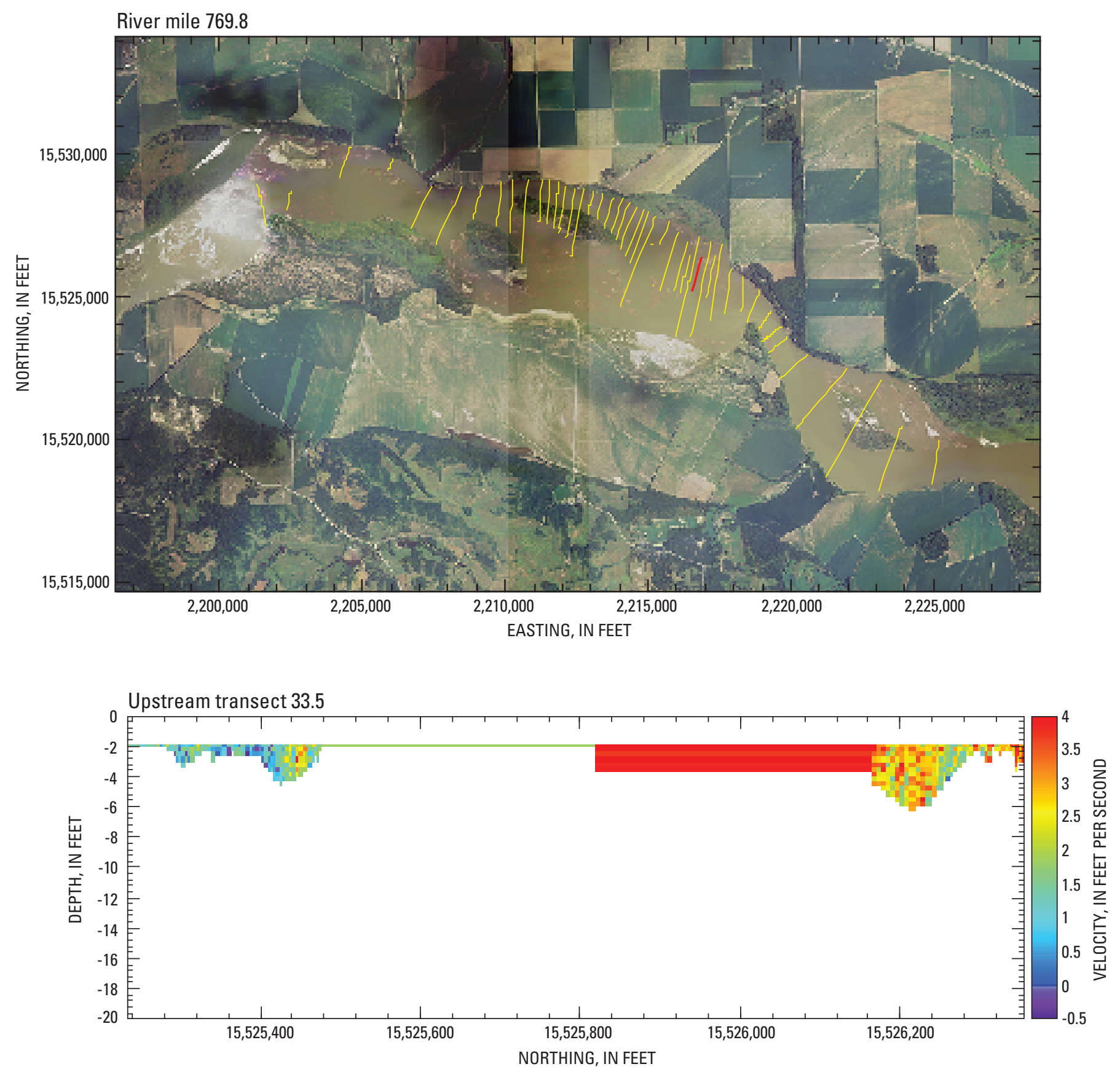

Base from Farm Service Agency digital orthophotography, 2003

Universal Transverse Mercator projection, Zone 14

Horizontal coordinates are referenced to the North American Datum of 1983 (NAD 83)

Figure B33. Location and velocity profile for upstream transect 33.5 . 

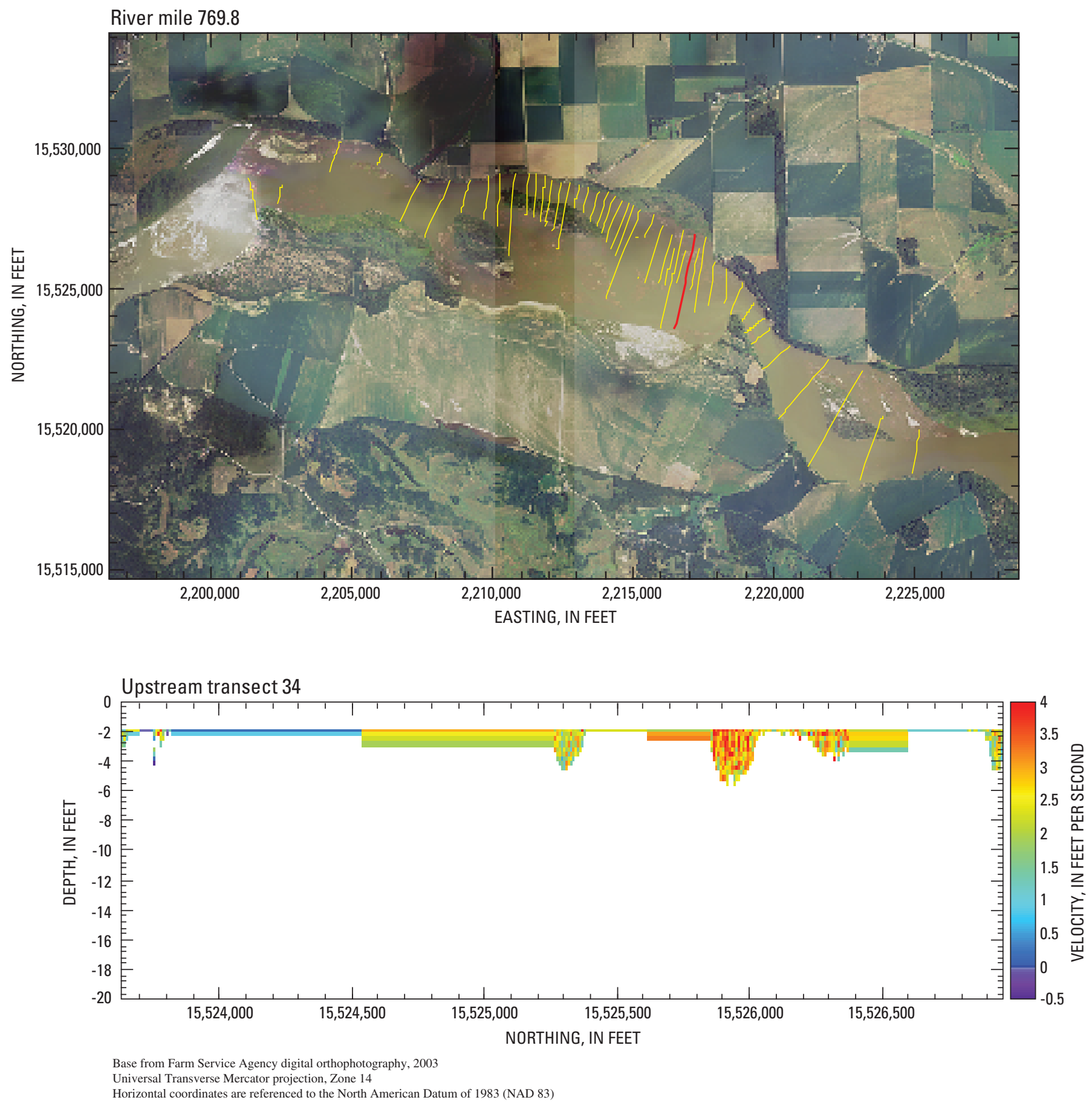

Figure B34. Location and velocity profile for upstream transect 34. 

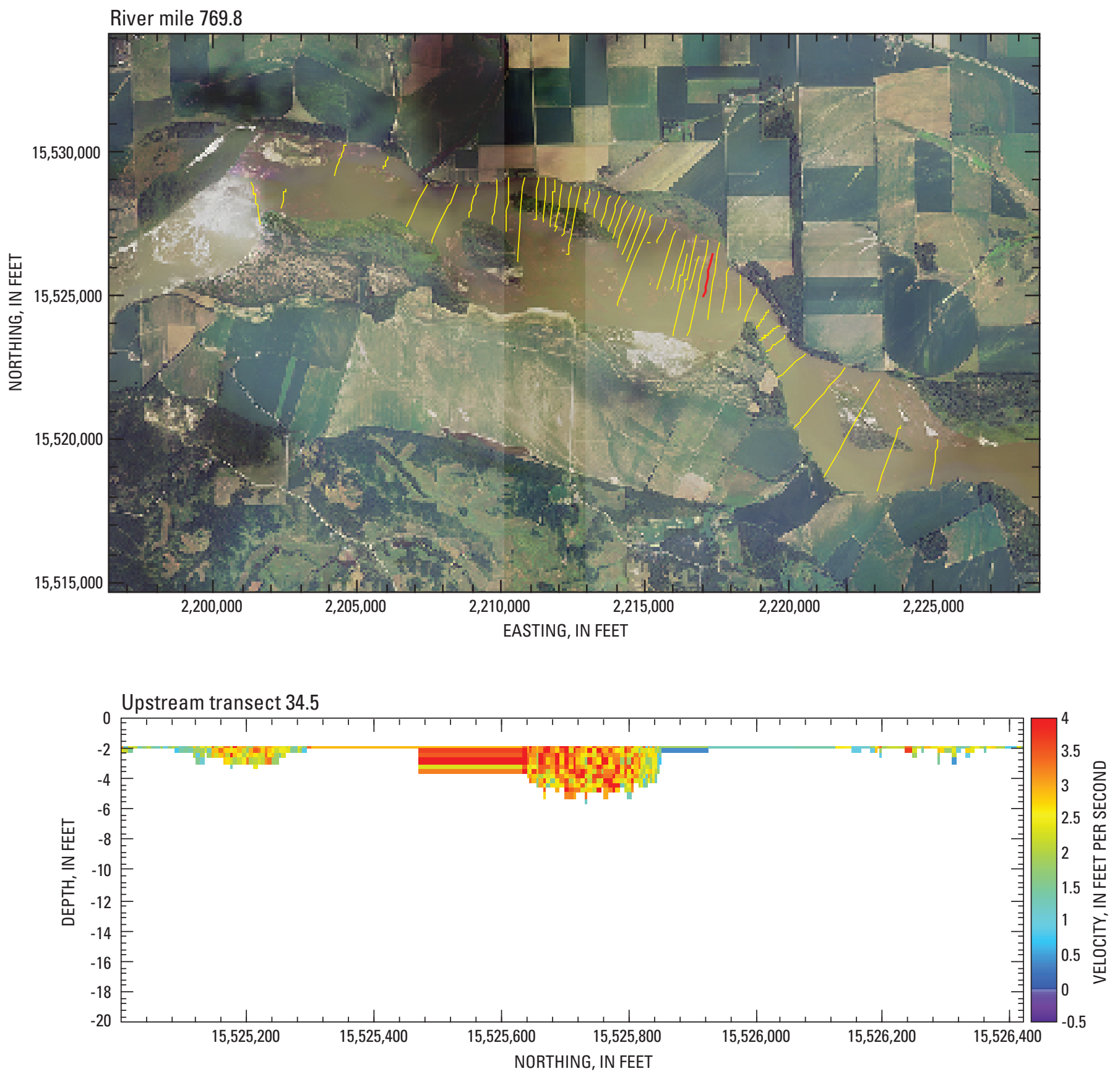

Base from Farm Service Agency digital orthophotography, 2003

Universal Transverse Mercator projection, Zone 14

Horizontal coordinates are referenced to the North American Datum of 1983 (NAD 83)

Figure B35. Location and velocity profile for upstream transect 34.5. 

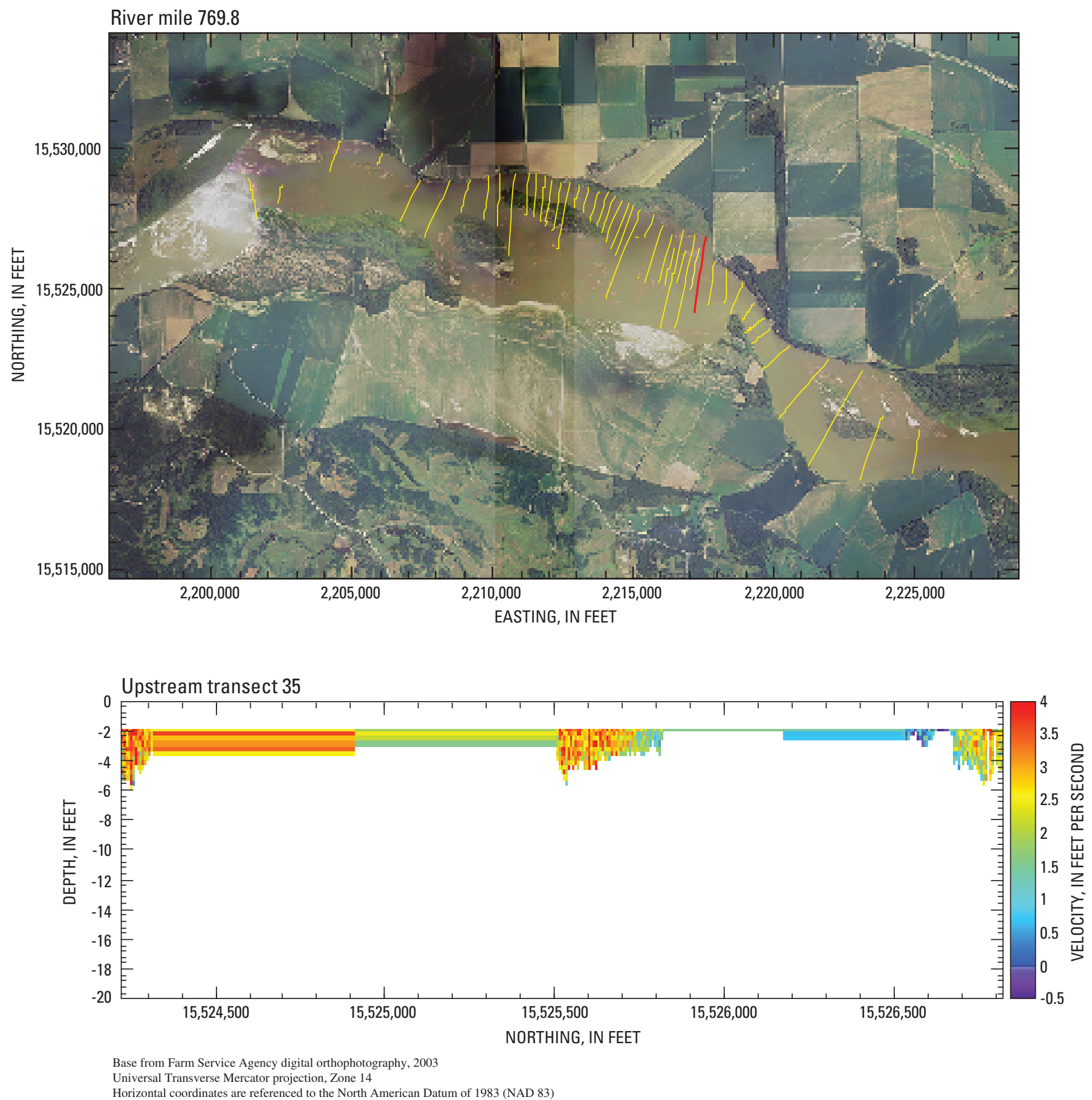

Figure B36. Location and velocity profile for upstream transect 35. 

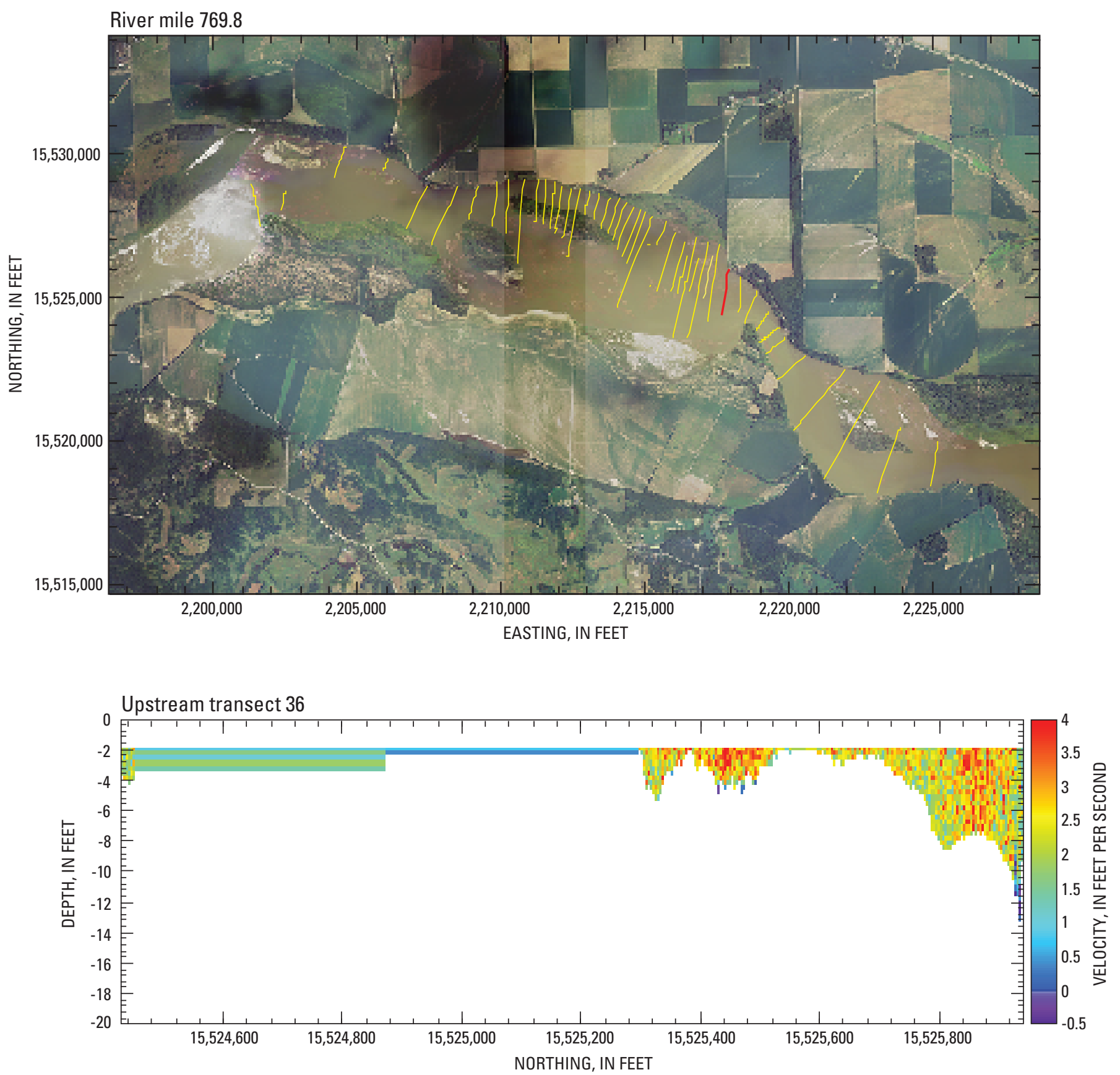

Base from Farm Service Agency digital orthophotography, 2003

Universal Transverse Mercator projection, Zone 14

Horizontal coordinates are referenced to the North American Datum of 1983 (NAD 83)

Figure B37. Location and velocity profile for upstream transect 36 . 

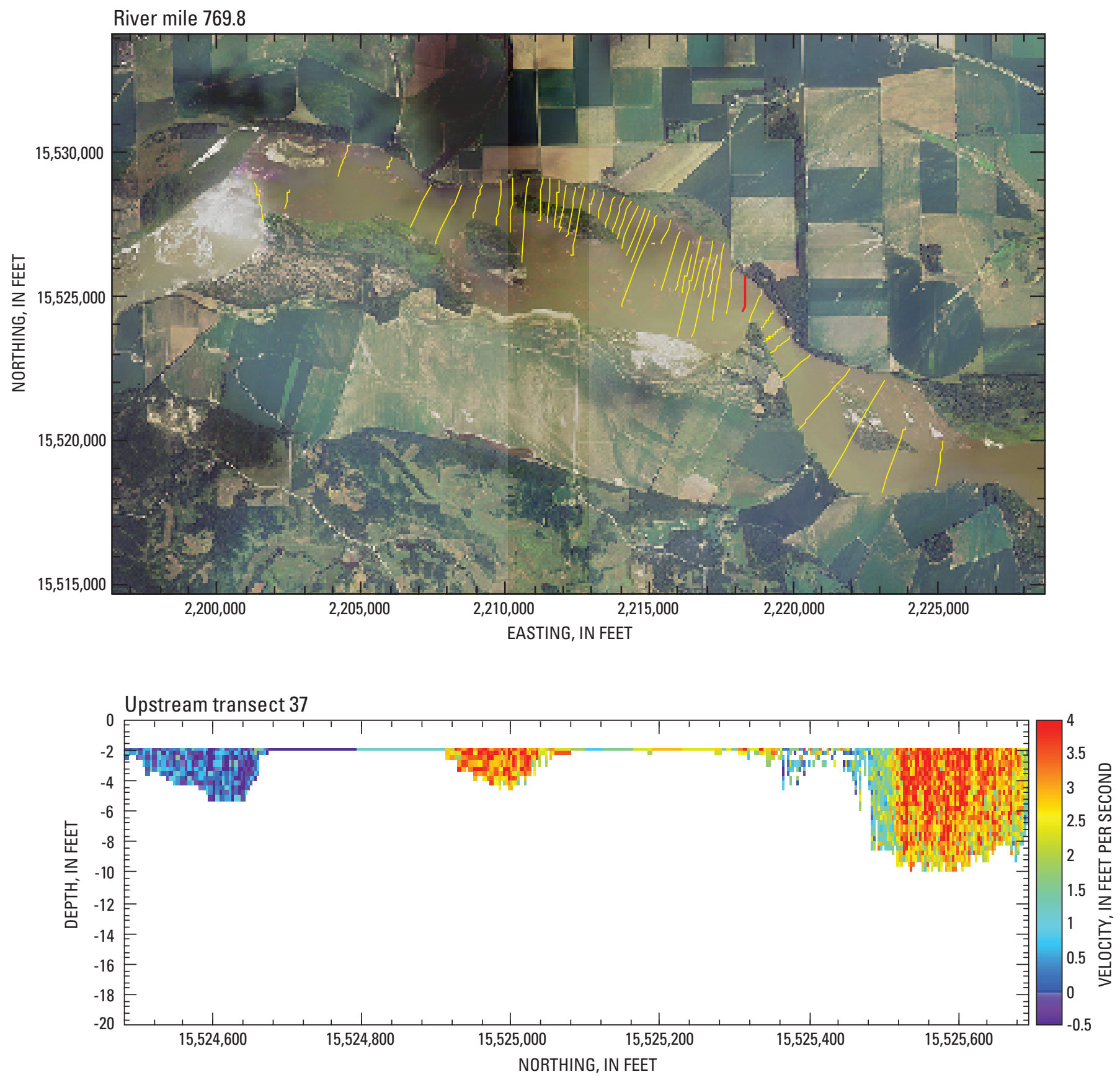

Base from Farm Service Agency digital orthophotography, 2003

Universal Transverse Mercator projection, Zone 14

Horizontal coordinates are referenced to the North American Datum of 1983 (NAD 83)

Figure B38. Location and velocity profile for upstream transect 37. 

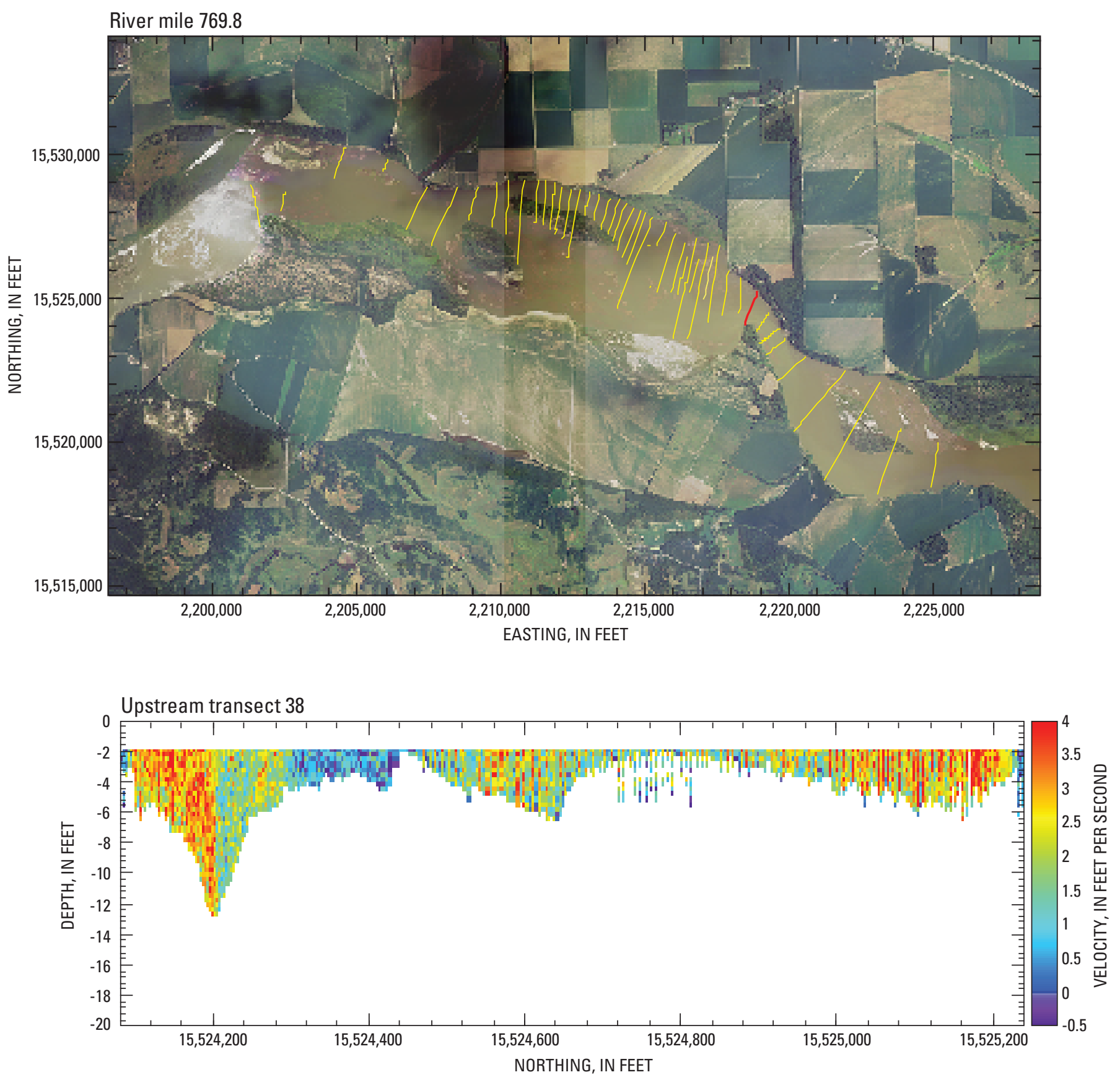

Base from Farm Service Agency digital orthophotography, 2003

Universal Transverse Mercator projection, Zone 14

Horizontal coordinates are referenced to the North American Datum of 1983 (NAD 83)

Figure B39. Location and velocity profile for upstream transect 38. 

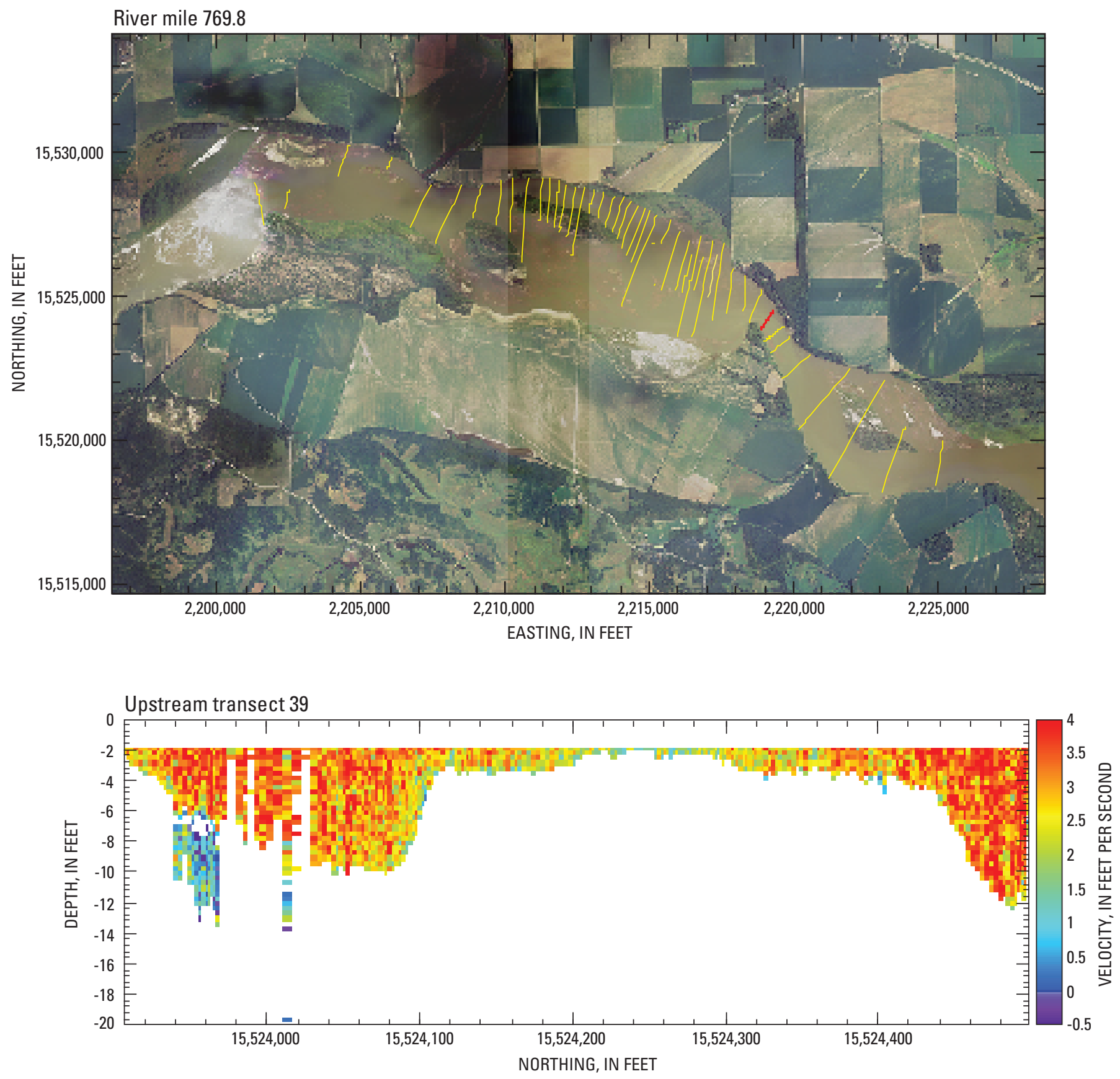

Base from Farm Service Agency digital orthophotography, 2003

Universal Transverse Mercator projection, Zone 14

Horizontal coordinates are referenced to the North American Datum of 1983 (NAD 83)

Figure B40. Location and velocity profile for upstream transect 39. 

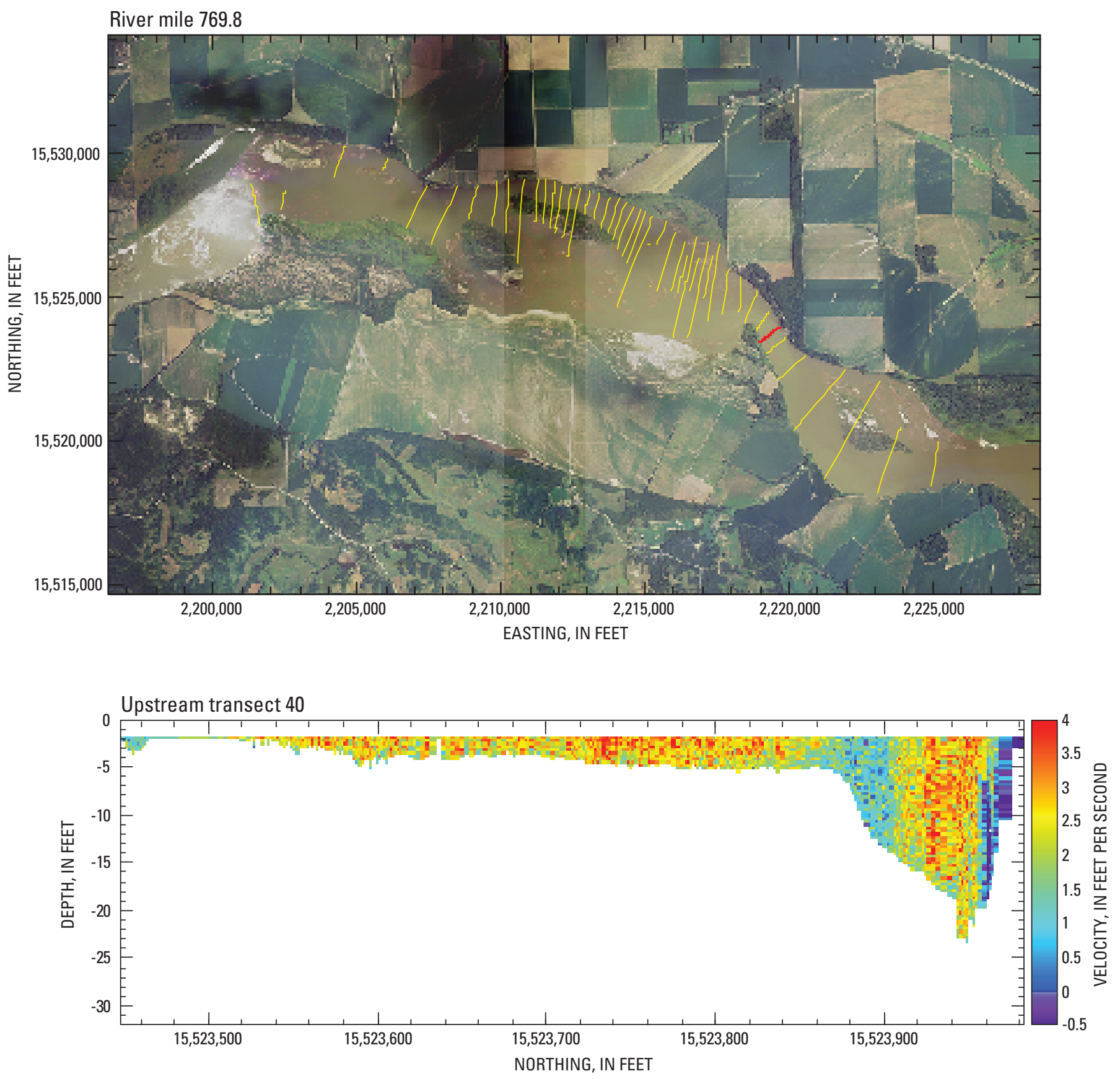

Base from Farm Service Agency digital orthophotography, 2003

Universal Transverse Mercator projection, Zone 14

Horizontal coordinates are referenced to the North American Datum of 1983 (NAD 83)

Figure B41. Location and velocity profile for upstream transect 40. 

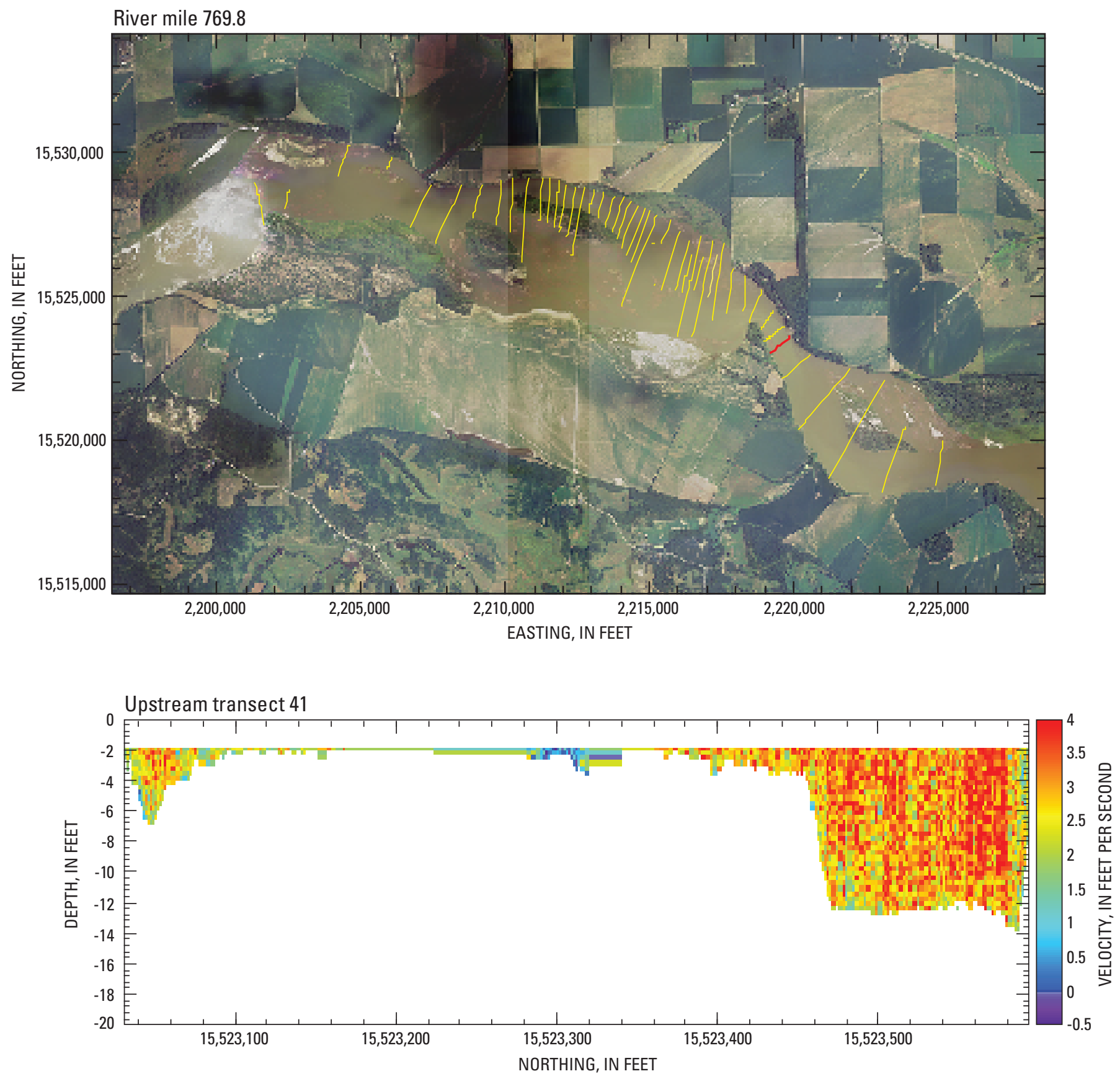

Base from Farm Service Agency digital orthophotography, 2003

Universal Transverse Mercator projection, Zone 14

Horizontal coordinates are referenced to the North American Datum of 1983 (NAD 83)

Figure B42. Location and velocity profile for upstream transect 41. 

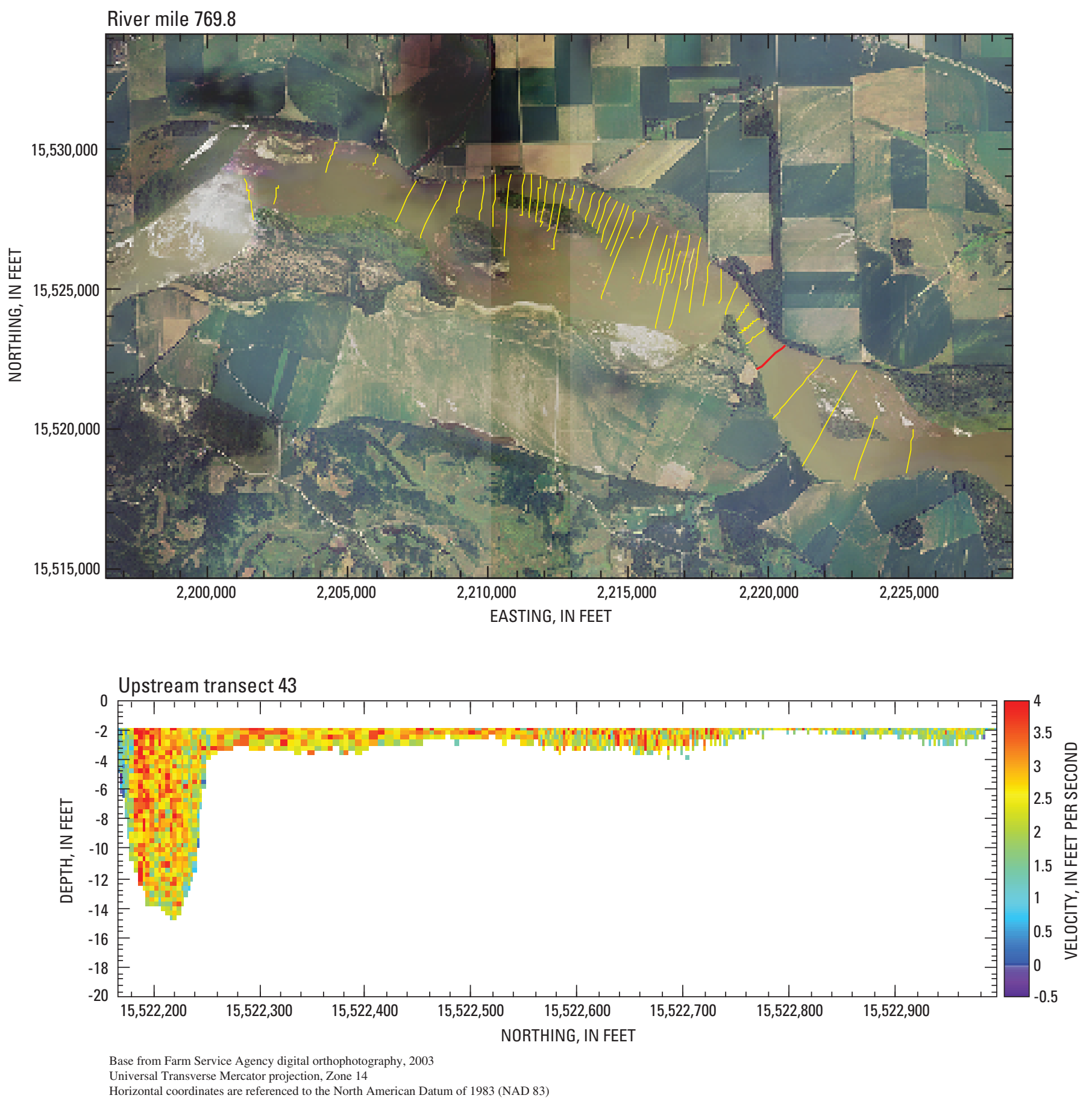

Figure B43. Location and velocity profile for upstream transect 43. 

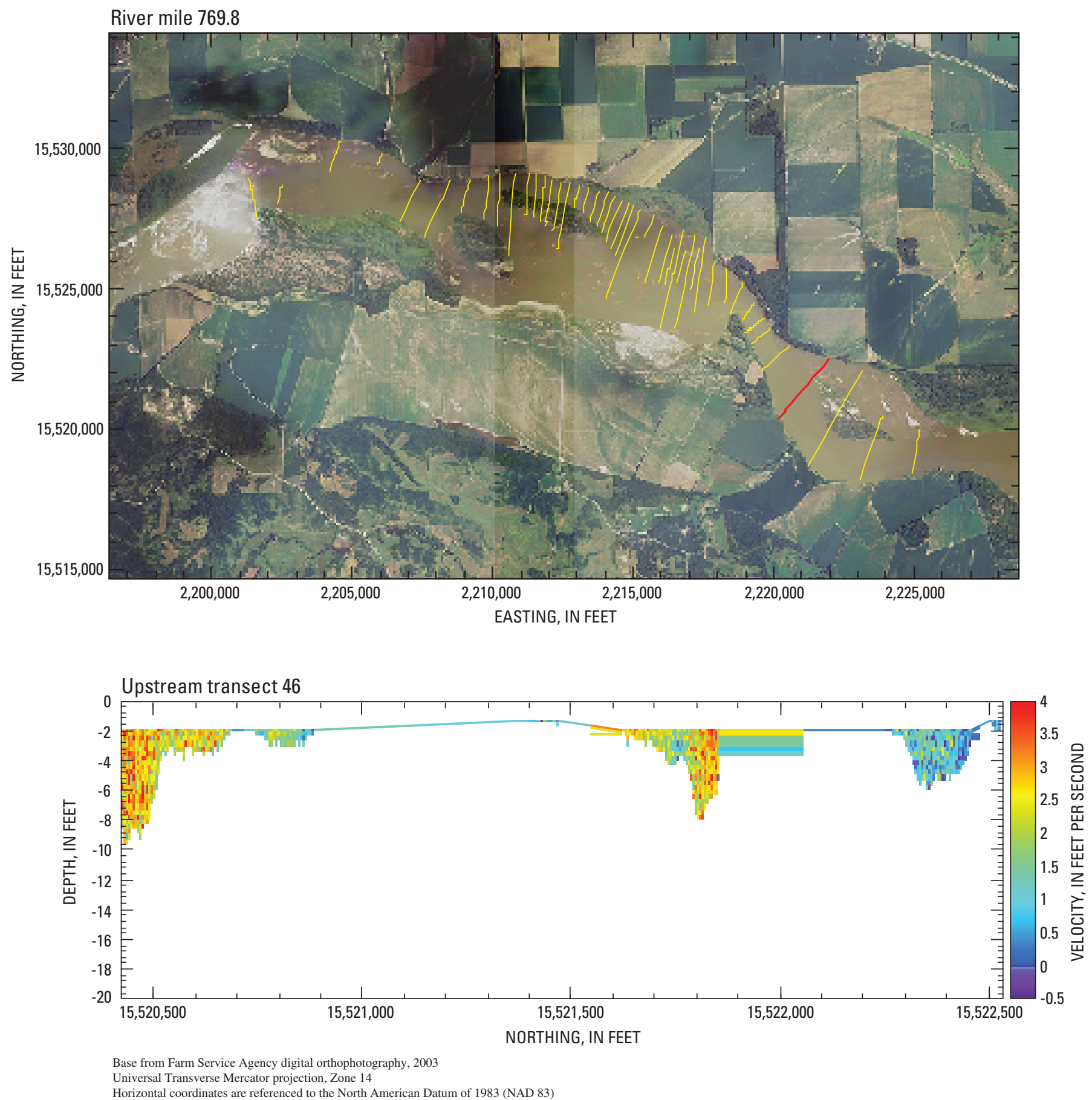

Figure B44. Location and velocity profile for upstream transect 46. 

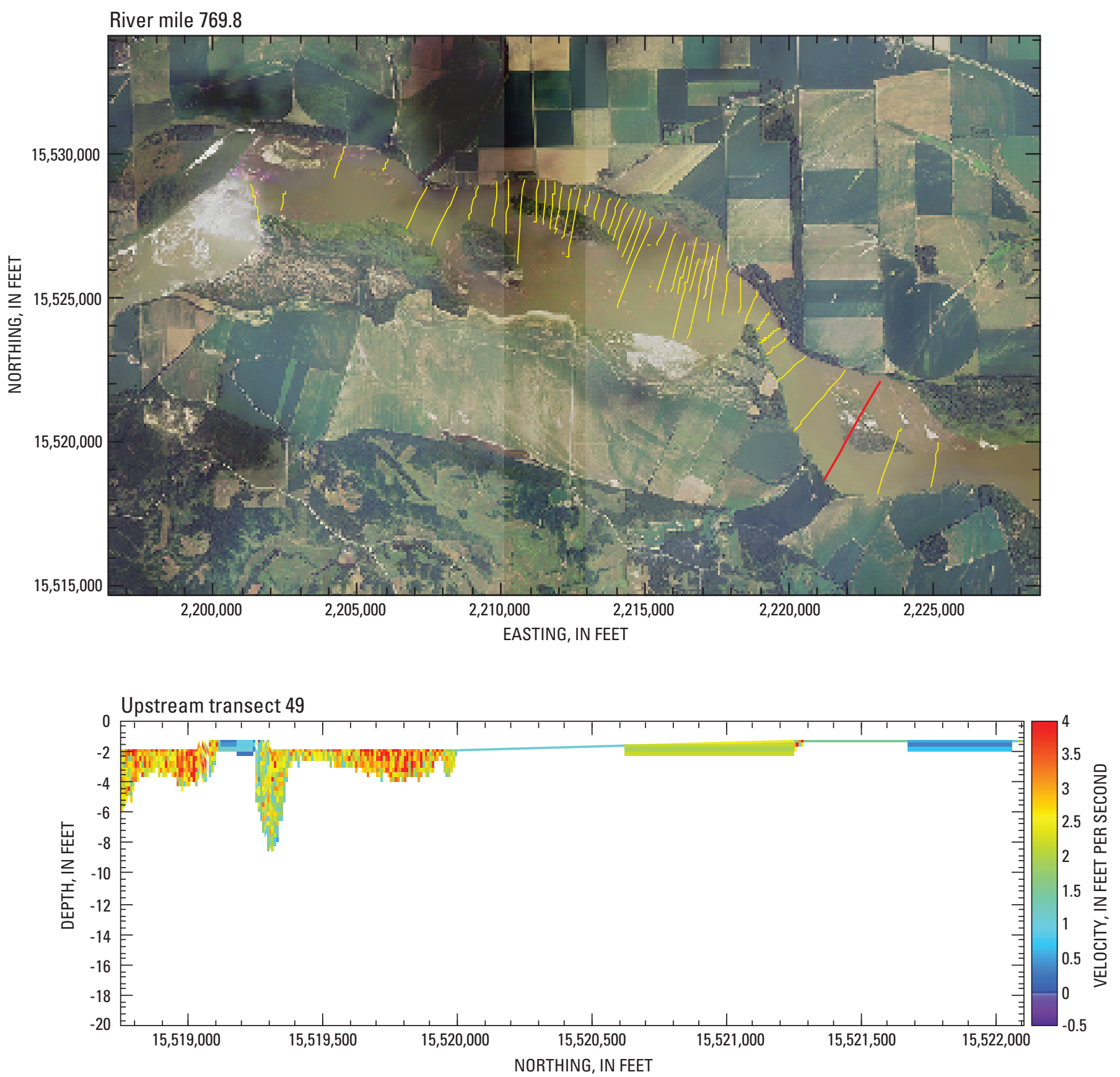

Base from Farm Service Agency digital orthophotography, 2003

Universal Transverse Mercator projection, Zone 14

Horizontal coordinates are referenced to the North American Datum of 1983 (NAD 83)

Figure B45. Location and velocity profile for upstream transect 49. 

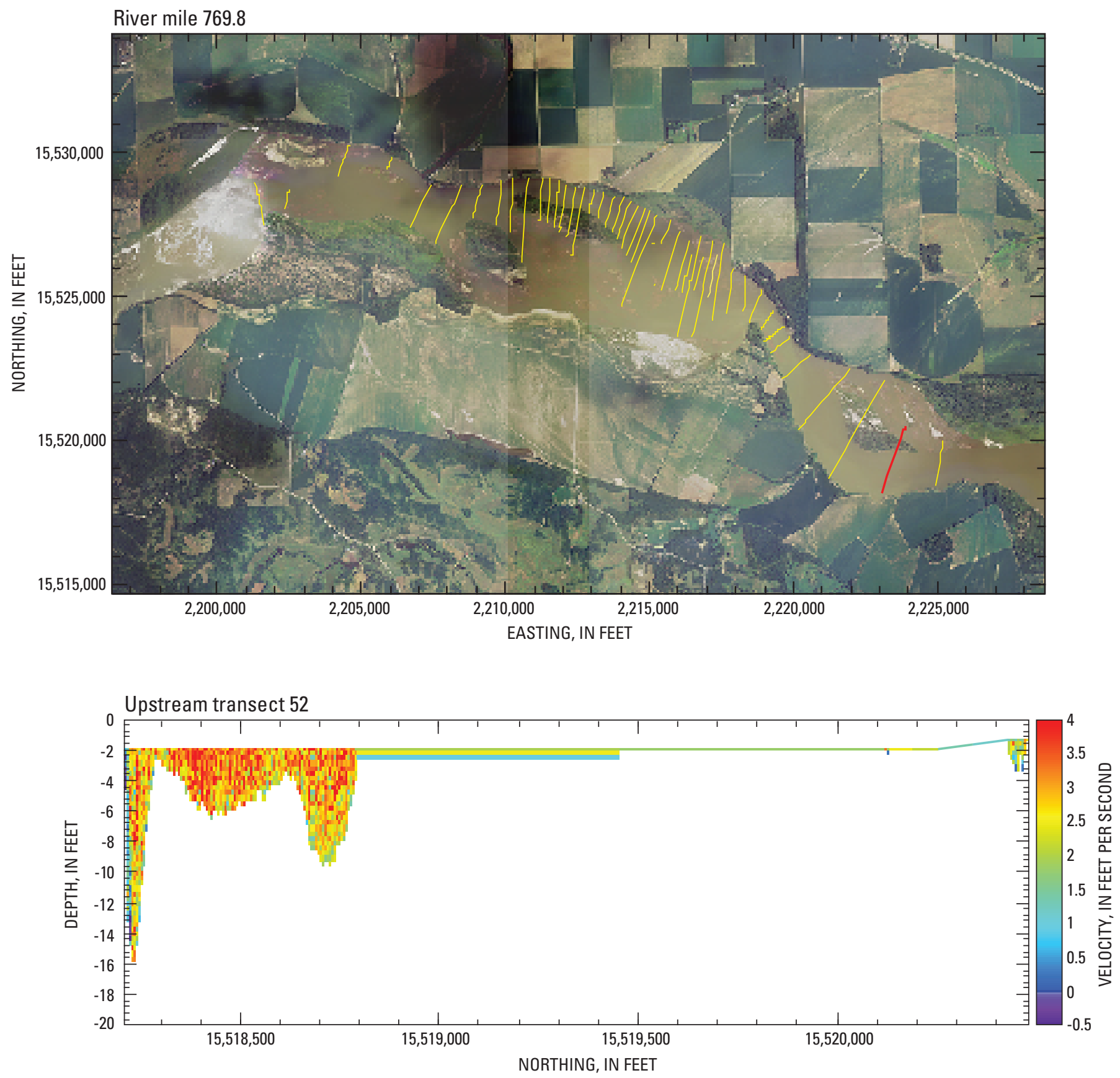

Base from Farm Service Agency digital orthophotography, 2003

Universal Transverse Mercator projection, Zone 14

Horizontal coordinates are referenced to the North American Datum of 1983 (NAD 83)

Figure B46. Location and velocity profile for upstream transect 52. 

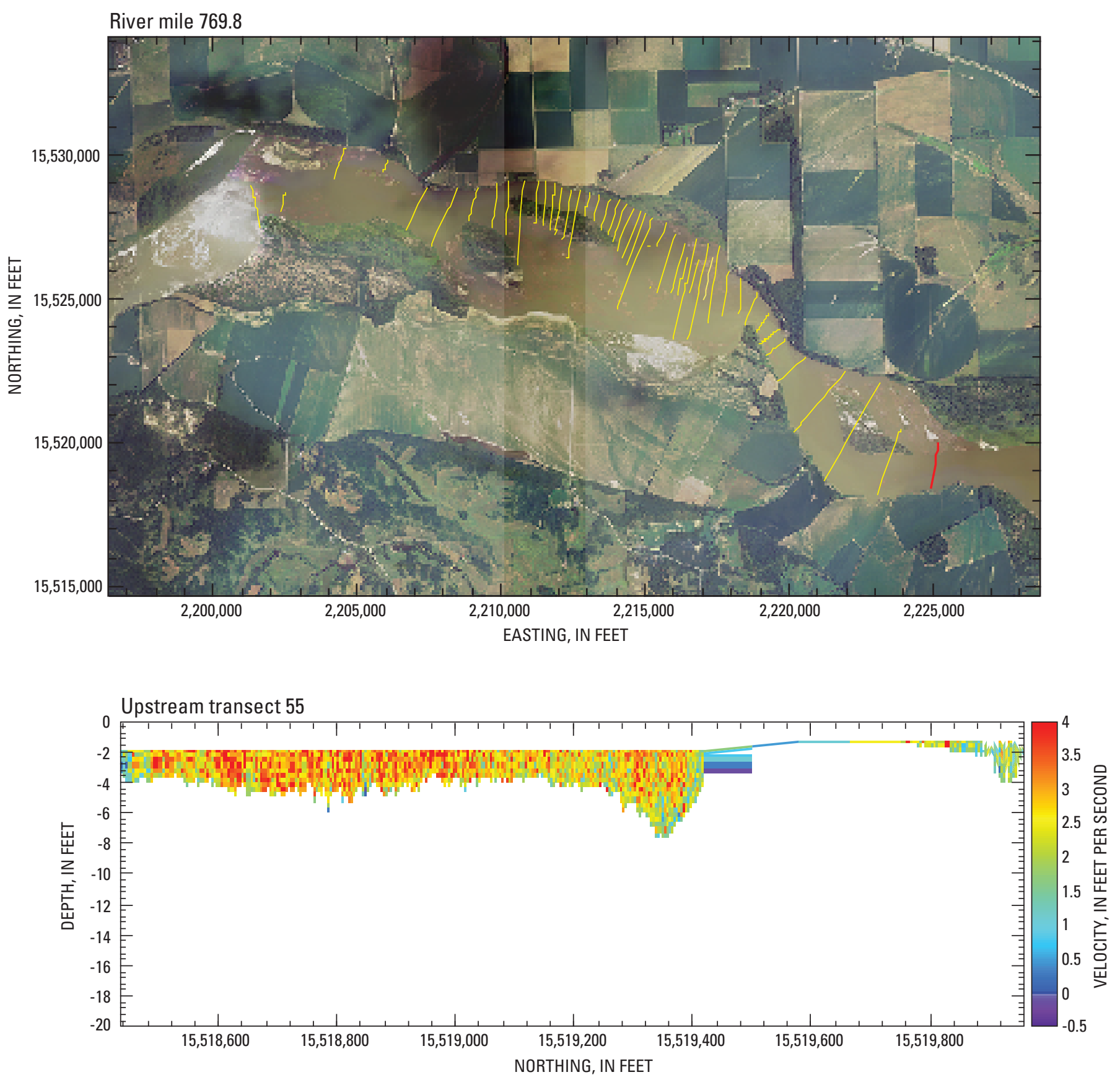

Base from Farm Service Agency digital orthophotography, 2003

Universal Transverse Mercator projection, Zone 14

Horizontal coordinates are referenced to the North American Datum of 1983 (NAD 83)

Figure B47. Location and velocity profile for upstream transect 55. 


\section{Section C. Data for Study Areas}

Data sets collected during this study are presented in two formats. Comma-delimited text files provide the data in a tabular format. Geospatial data sets are provided for use with GIS software. Metadata have been prepared for each geospatial data set to document the origin and nature of each data set. To access metadata, click the links below. A zipped file for the appropriate data set may then be downloaded from links within the metadata file. Once unzipped, both the comma-delimited text file and the geospatial data set are present. Postconstruction land-surface and surface-difference triangular irregular networks (TINs) of the study areas are available only as geospatial data sets.

\section{Data Sets}

- Transects [http://water.usgs.gov/GIS/metadatal usgswrd/XML/ofr07-1056_all_trans.shp.xml]

- Postconstruction

- Upstream bathymetry [http://water.usgs.gov/GIS/ metadata/usgswrd/XML/ofr07-1056_up_post_bathy. shp.xml]

- Upstream GPS [http://water.usgs.gov/GIS/metadata/ usgswrd/XML/ofr07-1056_up_post_gps.shp.xml]

- Upstream TIN [http://water.usgs.gov/GIS/metadata/ usgswrd/XML/ofro7-1056_up_post_tin.xml]

- Upstream surface difference TIN [http://water.usgs. gov/GIS/metadata/usgswrd/XML/ofro7-1056_up_ diff_tin.xml]

- Downstream bathymetry [http://water.usgs.gov/GIS/ metadata/usgswrd/XML/ofr07-1056_dn_post_bathy. shp.xml]

- Downstream GPS [http://water.usgs.gov/GIS/ metadata/usgswrd/XML/ofro7-1056_dn_post_gps. shp.xml]

- Downstream TIN [http://water.usgs.gov/GIS/ metadata/usgswrd/XML/ofr07-1056_dn_post_tin. $x m l]$
- Downstream surface difference TIN [http://water. usgs.gov/GIS/metadata/usgswrd/XML/ofr07-1056_ dn_diff_tin.xml]

- Velocity point values [http://water.usgs.gov/GIS/ metadata/usgswrd/XML/ofr07-1056_up_post_vel_ pt.shp.xml]

- ADCP velocity profiles [http://water.usgs.gov/GIS/ metadata/usgswrd/XML/ofro7-1056_up_post_vel_ array.shp.xml]

- Preconstruction

- Upstream bathymetry [http://water.usgs.gov/GIS/ metadata/usgswrd/XML/ofro7-1056_up_pre_bathy. shp.xml]

- Upstream GPS [http://water.usgs.gov/GIS/metadata/ usgswrd/XML/ofr07-1056_up_pre_gps.shp.xml]

- Downstream bathymetry [http://water.usgs.gov/GIS/ metadata/usgswrd/XML/ofro7-1056_dn_pre_bathy. shp.xml]

- Downstream GPS [http://water.usgs.gov/GIS/ metadata/usgswrd/XML/ofr07-1056_dn_pre_gps. shp.xml] 
Manuscript approved for publication, February 20, 2007.

Prepared by the Helena Publishing Service Center.

For more information concerning the research in this report, contact: U.S. Geological Survey

Director, South Dakota Water Science Center

1608 Mountain View Road

Rapid City, SD 57702

http://sd.water.usgs.gov/ 
